\title{
tert-Butyl Nitrite as a Twofold Hydrogen Abstractor for Dehydrogenative Coupling of Aldehydes with $\boldsymbol{N}$-Hydroxyimides
}

\author{
Peng-Fei Dai, ${ }^{\dagger \S}$ Yi-Ping Wang, ${ }^{\ddagger \S}$ Jian-Ping Qu, ${ }^{\dagger *}$ Yan-Biao Kang ${ }^{\ddagger *}$
}

\footnotetext{
$\dagger$ Institute of Advanced Synthesis, School of Chemistry and Molecular Engineering, Nanjing Tech University, Nanjing 211816, China

* Hefei National Laboratory for Physical Sciences at the Microscale, School of Chemistry and Materials Science, University of Science and Technology of China, Hefei 230026, China
}

E-mail: ias_jpqu@njtech.edu.cn, ybkang@ustc.edu.cn

\section{Contents}

1. General information $\quad$ S1

2. Experimental procedures $\quad \mathrm{S} 1$

3. References $\quad$ S18

$\begin{array}{ll}\text { 4. NMR spectra } & \text { S19-S116 }\end{array}$

\section{General information}

Solvents were pre-dried over activated $4 \AA$ molecular sieves and heated to reflux over calcium hydride $\left(\mathrm{CH}_{3} \mathrm{CN}\right)$ under argon atmosphere and collected by distillation. ${ }^{1} \mathrm{H},{ }^{13} \mathrm{C}\left\{{ }^{1} \mathrm{H}\right\} \mathrm{NMR}$ spectra were recorded on a Bruker 400 spectrometer; Chemical shifts are reported in $\delta$ units relative to $\mathrm{CDCl}_{3}$ $\left[{ }^{1} \mathrm{H} \delta=7.26,{ }^{13} \mathrm{C} \delta=77.36\right]$. HRMS were recorded by the mass spectrometry service at University of Science and Technology of China. Aldehydes and other chemicals without notes in experimental section were purchased from commercial sources.

\section{Experimental procedures}

\subsection{General Procedure for Preparation of ${ }^{t} \mathrm{BuONO}$}

To an ice-cold solution of $(c) \mathrm{H}_{2} \mathrm{SO}_{4}(22 \mathrm{~mL}, 0.4 \mathrm{~mol})$ in $\mathrm{H}_{2} \mathrm{O}(25 \mathrm{~mL})$ was added $t$ - $\mathrm{BuOH}$ ( $38 \mathrm{~mL}$, $0.4 \mathrm{~mol})$ dropwise followed by the addition of a solution of sodium nitrite $\left(42 \mathrm{~g}\right.$, in $100 \mathrm{~mL}$ of $\left.\mathrm{H}_{2} \mathrm{O}\right)$. The reaction was kept at $0{ }^{\circ} \mathrm{C}$ during the addition, which was completed after $1 \mathrm{~h}$. The reaction mixture was warmed to r.t. and stirred overnight. The aquous layer was separated and the organic portion was washed with saturated aq. $\mathrm{NaHCO}_{3}$ solution and saturated aq. $\mathrm{NaCl}$ solution. The organic portion was then dried over $\mathrm{Na}_{2} \mathrm{SO}_{4}$ and purified by distillation. A light yellow fraction (ca. 20-30 mL) was collected at $80-90{ }^{\circ} \mathrm{C}$ and used for all dehydrogenative coupling of aldehydes with $N$-hydroxyimides 
reactions. It was stored at $4{ }^{\circ} \mathrm{C}$.

\subsection{Optimization of Reaction Conditions}

$N$-hydroxyphthalimide (NHPI) (82 mg, $0.5 \mathrm{mmol}, 1.0$ equiv) was weighed directly into a Schlenk tube equipped with a magnetic stir bar. After dried in vacuo for $15 \mathrm{~min}$ and charged with nitrogen gas, dry $\mathrm{MeCN}(2 \mathrm{~mL})$ was added. Then aldehyde (x mmol) was added dropwise into the mixture. ${ }^{t} \mathrm{BuONO}$ (TBN) (z mmol) was last added and the tube was capped with a glass cap. The resulting solution was stirred at $25-80{ }^{\circ} \mathrm{C}$ for 12 or $24 \mathrm{~h}$. After cooling to rt, the reaction mixture was diluted with $\mathrm{CH}_{2} \mathrm{Cl}_{2}$ (2 $\mathrm{mL}$ ), the solvent was removed under vacuum and the residue was diluted with $\mathrm{CHCl}_{3}$. And the conversions was determined by ${ }^{1} \mathrm{H}$ NMR technology using nitromethane $\left(\mathrm{MeNO}_{2}\right)$ and tert-butyl methyl ether ( $\left.{ }^{t} \mathrm{BuOMe}\right)$ as internal standard.

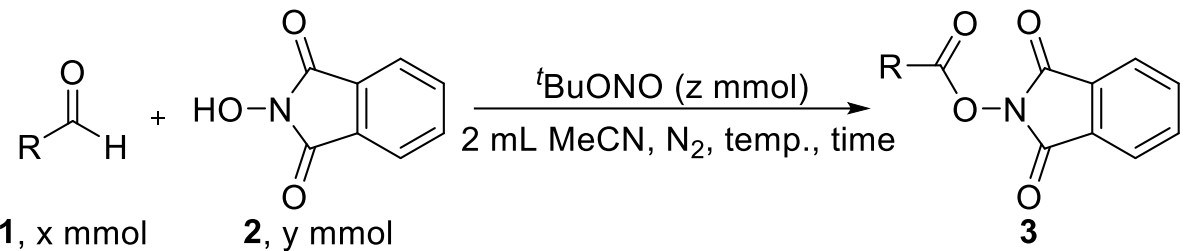

\begin{tabular}{cccccc}
\hline entry $^{a}$ & $\mathrm{R}$ & $\mathrm{x}$ & $\mathrm{y}$ & $\mathrm{z}$ & $3(\%)^{b}$ \\
\hline 1 & Cyclohexyl & 0.5 & 0.5 & 0.5 & 80 \\
2 & Cyclohexyl & 0.6 & 0.5 & 0.5 & 91 \\
3 & Cyclohexyl & 0.65 & 0.5 & 0.5 & 92 \\
4 & Cyclohexyl & 0.75 & 0.5 & 0.5 & quant. \\
5 & 4-Methoxylbenzyl & 0.75 & 0.5 & 0.5 & 36 \\
$6^{c}$ & 4-Methoxylbenzyl & 0.75 & 0.5 & 0.5 & 66 \\
$7^{d}$ & 4-Methoxylbenzyl & 0.75 & 0.5 & 0.5 & 87 \\
$8^{e}$ & 4-Methoxylbenzyl & 0.75 & 0.5 & 0.5 & $93^{f}$ \\
\hline
\end{tabular}

${ }^{a}$ Conditions: 1 (x mmol), 2 (y mmol), ${ }^{t} \mathrm{BuONO}(\mathrm{z} \mathrm{mmol}), \mathrm{MeCN}(2 \mathrm{~mL}), 25{ }^{\circ} \mathrm{C}$, nitrogen, 12 h. ${ }^{b}$ Conversions determined by ${ }^{1} \mathrm{H}$ NMR using $\mathrm{CH}_{3} \mathrm{NO}_{2}$ and ${ }^{t} \mathrm{BuOMe}$ as internal standard. ${ }^{c} 50{ }^{\circ} \mathrm{C} .{ }^{d} 60$ ${ }^{\circ} \mathrm{C} .{ }^{e} 80{ }^{\circ} \mathrm{C}, 24$ h. ${ }^{f}$ Isolated yield.

\subsection{General Procedure for Preparation of $N$-Hydroxyphthalimide Esters from Aldehydes}

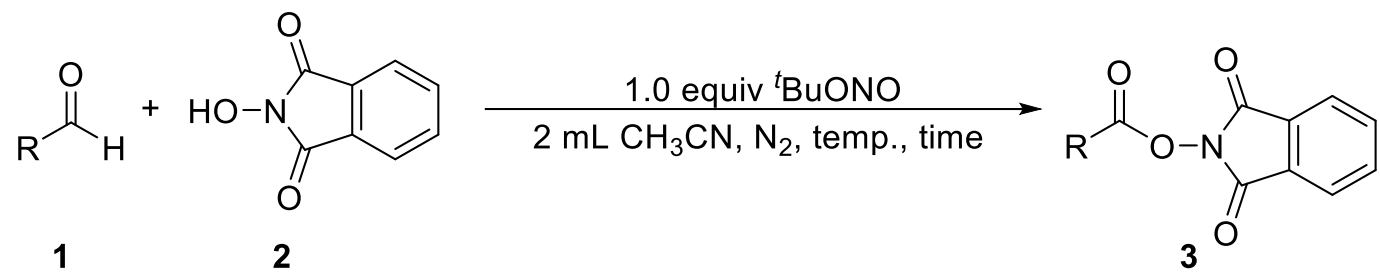

$N$-hydroxyphthalimide (NHPI) ( $82 \mathrm{mg}, 0.5 \mathrm{mmol}, 1.0$ equiv) was weighed directly into a Schlenk tube equipped with a magnetic stir bar. After dried in vacuo for $15 \mathrm{~min}$ and charged with nitrogen gas, dry $\mathrm{MeCN}(2 \mathrm{~mL})$ was added. Then aldehyde $(0.75 \mathrm{mmol}, 1.5$ equiv) was added dropwise into the 
mixture. ${ }^{t} \mathrm{BuONO}(\mathrm{TBN})(60 \mu \mathrm{L}, 0.5 \mathrm{mmol}, 1.0$ equiv) was last added and the tube was capped with a glass cap. The resulting solution was stirred at $25-80^{\circ} \mathrm{C}$ for 12 or $24 \mathrm{~h}$. After cooling to rt, the reaction mixture was diluted with $\mathrm{CH}_{2} \mathrm{Cl}_{2}(2 \mathrm{~mL})$, the solvent was removed under vacuum and the residue was purified by column chromatography on silica gel to give the pure product.

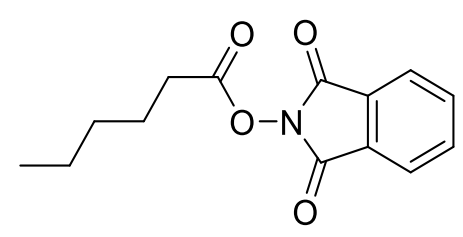

\section{1,3-Dioxoisoindolin-2-yl hexanoate (3a) ${ }^{1}$}

Prepared according to the general procedure, $25^{\circ} \mathrm{C}, 12 \mathrm{~h}$. Purification was performed by flash column chromatography (PE/EA/DCM = 20:1:1), colorless oil liquid (116.3 mg, 89\% yield). ${ }^{1} \mathrm{H}$ NMR $\left(\mathrm{CDCl}_{3}, 400 \mathrm{MHz}\right): \delta 7.89(\mathrm{dd}, J=4.8,3.2 \mathrm{~Hz}, 2 \mathrm{H}), 7.79(\mathrm{dd}, J=4.8,2.8 \mathrm{~Hz}, 2 \mathrm{H}), 2.67(\mathrm{t}, J=7.2$ $\mathrm{Hz}, 2 \mathrm{H}), 1.80(\mathrm{t}, J=7.2 \mathrm{~Hz}, 2 \mathrm{H}), 1.46-1.32(\mathrm{~m}, 4 \mathrm{H}), 0.94(\mathrm{t}, J=7.2 \mathrm{~Hz}, 3 \mathrm{H}) .{ }^{13} \mathrm{C}\left\{{ }^{1} \mathrm{H}\right\} \mathrm{NMR}\left(\mathrm{CDCl}_{3}\right.$, $100 \mathrm{MHz}): \delta 170.0,162.3,135.1,129.3,124.3,31.3,24.7,22.5,14.2$.

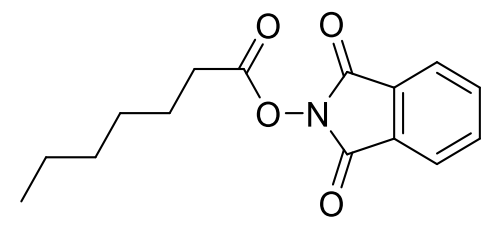

\section{1,3-Dioxoisoindolin-2-yl heptanoate (3b) ${ }^{1}$}

Prepared according to the general procedure, $25^{\circ} \mathrm{C}, 12 \mathrm{~h}$. Purification was performed by flash column chromatography $(\mathrm{PE} / \mathrm{EA} / \mathrm{DCM}=20: 1: 1)$, white solid $(126.6 \mathrm{mg}, 92 \%$ yield $) .{ }^{1} \mathrm{H} \mathrm{NMR}\left(\mathrm{CDCl}_{3}\right.$, $400 \mathrm{MHz}): \delta 7.90(\mathrm{dd}, J=5.2,3.2 \mathrm{~Hz}, 2 \mathrm{H}), 7.80(\mathrm{dd}, J=5.2,3.2 \mathrm{~Hz}, 2 \mathrm{H}), 2.68(\mathrm{t}, J=7.6 \mathrm{~Hz}, 2 \mathrm{H})$, 1.82-1.75 (m, $2 \mathrm{H}), 1.46-1.43(\mathrm{~m}, 2 \mathrm{H}), 1.35-1.33(\mathrm{~m}, 4 \mathrm{H}), 0.92(\mathrm{t}, J=6.0 \mathrm{~Hz}, 3 \mathrm{H}) .{ }^{13} \mathrm{C}\left\{{ }^{1} \mathrm{H}\right\} \mathrm{NMR}$ $\left(\mathrm{CDCl}_{3}, 100 \mathrm{MHz}\right): \delta$ 170.0, 162.4, 135.1, 129.3, 124.3, 31.6, 31.4, 28.8, 25.0, 22.7, 14.3.<smiles>O=C(CCc1ccccc1)ON1C(=O)c2ccccc2C1=O</smiles>

\section{1,3-Dioxoisoindolin-2-yl 3-phenylpropanoate (3c) ${ }^{2}$}

Prepared according to the general procedure, $50{ }^{\circ} \mathrm{C}, 12 \mathrm{~h}$. Purification was performed by flash column chromatography $(\mathrm{PE} / \mathrm{EA} / \mathrm{DCM}=20: 1: 1)$, white solid $(146.1 \mathrm{mg}, 99 \%$ yield $) .{ }^{1} \mathrm{H} \mathrm{NMR}\left(\mathrm{CDCl}_{3}\right.$, $400 \mathrm{MHz}$ ): $\delta$ 7.91-7.89 (m, $2 \mathrm{H}), 7.81-7.79(\mathrm{~m}, 2 \mathrm{H}), 7.36(\mathrm{t}, J=7.6 \mathrm{~Hz}, 2 \mathrm{H})$, 7.27-7.23 (m, $3 \mathrm{H})$, $3.13(\mathrm{t}, J=7.6 \mathrm{~Hz}, 2 \mathrm{H}), 3.01(\mathrm{t}, J=7.6 \mathrm{~Hz}, 2 \mathrm{H}) .{ }^{13} \mathrm{C}\left\{{ }^{1} \mathrm{H}\right\} \mathrm{NMR}\left(\mathrm{CDCl}_{3}, 100 \mathrm{MHz}\right): \delta 169.2,162.3$, $139.5,135.1,129.3,129.1,128.7,127.1,124.3,33.1,30.9$.<smiles>CC(C)C(=O)ON1C(=O)c2ccccc2C1=O</smiles> 
Prepared according to the general procedure, $25^{\circ} \mathrm{C}, 24 \mathrm{~h}$. Purification was performed by flash column chromatography $(\mathrm{PE} / \mathrm{EA} / \mathrm{DCM}=20: 1: 1)$, white solid $(115.4 \mathrm{mg}, 99 \%$ yield $) .{ }^{1} \mathrm{H} \mathrm{NMR}\left(\mathrm{CDCl}_{3}\right.$, $400 \mathrm{MHz}): \delta 7.90$ (dd, $J=4.8,2.4 \mathrm{~Hz}, 2 \mathrm{H}), 7.80(\mathrm{dd}, J=4.8,2.4 \mathrm{~Hz}, 2 \mathrm{H}), 3.01-2.93$ (m, $1 \mathrm{H}), 1.39$ $(\mathrm{d}, J=6.8 \mathrm{~Hz}, 6 \mathrm{H}) .{ }^{13} \mathrm{C}\left\{{ }^{1} \mathrm{H}\right\} \mathrm{NMR}\left(\mathrm{CDCl}_{3}, 100 \mathrm{MHz}\right): \delta 173.4,162.4,135.1,129.4,124.3,32.1,19.2$.<smiles>CCC(C)C(=O)ON1C(=O)c2ccccc2C1=O</smiles>

\section{1,3-Dioxoisoindolin-2-yl 2-methylbutanoate $(3 e)^{3}$}

Prepared according to the general procedure, $25^{\circ} \mathrm{C}, 12 \mathrm{~h}$. Purification was performed by flash column chromatography (PE/EA/DCM = 20:1:1), colorless oil liquid (118.6 mg, 96\% yield). ${ }^{1} \mathrm{H}$ NMR $\left(\mathrm{CDCl}_{3}, 400 \mathrm{MHz}\right): \delta 7.89(\mathrm{dd}, J=5.2,2.8 \mathrm{~Hz}, 2 \mathrm{H}), 7.79(\mathrm{dd}, J=5.2,2.8 \mathrm{~Hz}, 2 \mathrm{H}), 2.83-2.74(\mathrm{~m}, 1$ H), 1.91-1.80 (m, $1 \mathrm{H}), 1.75-1.64(\mathrm{~m}, 1 \mathrm{H}), 1.36(\mathrm{~d}, J=7.2 \mathrm{~Hz}, 3 \mathrm{H}), 1.09(\mathrm{t}, J=7.6 \mathrm{~Hz}, 3 \mathrm{H})$. ${ }^{13} \mathrm{C}\left\{{ }^{1} \mathrm{H}\right\} \mathrm{NMR}\left(\mathrm{CDCl}_{3}, 100 \mathrm{MHz}\right): \delta 173.0,162.4,135.0,129.3,124.3,38.9,27.2,16.8,11.6$.

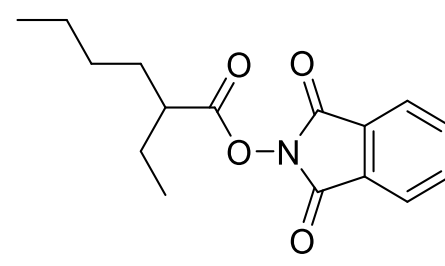

\section{1,3-Dioxoisoindolin-2-yl 2-ethylhexanoate $(3 f)^{3}$}

Prepared according to the general procedure, $25{ }^{\circ} \mathrm{C}, 24 \mathrm{~h}$. Purification was performed by flash column chromatography $(\mathrm{PE} / \mathrm{EA} / \mathrm{DCM}=20: 1: 1)$, colorless oil liquid $(141.7 \mathrm{mg}, 98 \%$ yield $) .{ }^{1} \mathrm{H}$ NMR $\left(\mathrm{CDCl}_{3}, 400 \mathrm{MHz}\right): \delta$ 7.88-7.86 (m, $\left.2 \mathrm{H}\right), 7.79-7.77$ (m, $\left.2 \mathrm{H}\right), 2.69-2.62(\mathrm{~m}, 1 \mathrm{H}), 1.84-1.59(\mathrm{~m}, 4 \mathrm{H})$, 1.48-1.34 (m, $4 \mathrm{H}), 1.09$ (t, $J=7.2 \mathrm{~Hz}, 3 \mathrm{H}), 0.95(\mathrm{t}, J=7.2 \mathrm{~Hz}, 3 \mathrm{H}) .{ }^{13} \mathrm{C}\left\{{ }^{1} \mathrm{H}\right\} \mathrm{NMR}\left(\mathrm{CDCl}_{3}, 100\right.$ MHz): $\delta 172.8,162.4,135.0,129.4,124.2,45.2,32.1,29.6,26.0,22.9,14.2,11.9$.<smiles>O=C(ON1C(=O)c2ccccc2C1=O)C1CC1</smiles>

\section{1,3-Dioxoisoindolin-2-yl cyclopropanecarboxylate (3g) ${ }^{4}$}

Prepared according to the general procedure, $50{ }^{\circ} \mathrm{C}, 24 \mathrm{~h}$. Purification was performed by flash column chromatography $(\mathrm{PE} / \mathrm{EA} / \mathrm{DCM}=20: 1: 1)$, white solid (114.4 mg, 99\% yield). ${ }^{1} \mathrm{H} \mathrm{NMR}\left(\mathrm{CDCl}_{3}\right.$, $400 \mathrm{MHz}): \delta 7.89(\mathrm{dd}, J=5.2,2.8 \mathrm{~Hz}, 2 \mathrm{H}), 7.79$ (dd, $J=5.2,3.2 \mathrm{~Hz}, 2 \mathrm{H}), 1.99-1.93$ (m, $1 \mathrm{H}), 1.29-$ $1.24(\mathrm{~m}, 2 \mathrm{H}), 1.22-1.15(\mathrm{~m}, 2 \mathrm{H}) .{ }^{13} \mathrm{C}\left\{{ }^{1} \mathrm{H}\right\} \mathrm{NMR}\left(\mathrm{CDCl}_{3}, 100 \mathrm{MHz}\right): \delta 171.5,162.4,135.1,129.3$, $124.3,10.9,10.6$.<smiles>O=C(ON1C(=O)c2ccccc2C1=O)C1CCCC1</smiles> 
Prepared according to the general procedure, $50{ }^{\circ} \mathrm{C}, 24 \mathrm{~h}$. Purification was performed by flash column chromatography (PE/EA/DCM =20:1:1), white solid (128.2 mg, 99\% yield). ${ }^{1} \mathrm{H} \mathrm{NMR}\left(\mathrm{CDCl}_{3}\right.$, $400 \mathrm{MHz}$ ): $\delta$ 7.89-7.87 (m, 2 H), 7.79-7.77 (m, 2 H), 3.16-3.08 (m, $1 \mathrm{H}), 2.13-1.99$ (m, 4 H), $1.83-$ 1.75 (m, $2 \mathrm{H}), 1.72-1.66(\mathrm{~m}, 2 \mathrm{H}) .{ }^{13} \mathrm{C}\left\{{ }^{1} \mathrm{H}\right\} \mathrm{NMR}\left(\mathrm{CDCl}_{3}, 100 \mathrm{MHz}\right): \delta 173.2,162.5,135.0,129.4$, $124.3,41.0,30.6,26.3$.

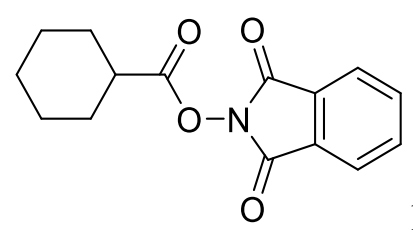

\section{1,3-Dioxoisoindolin-2-yl cyclohexanecarboxylate $(3 i)^{2}$}

Prepared according to the general procedure, $25^{\circ} \mathrm{C}, 12 \mathrm{~h}$. Purification was performed by flash column chromatography (PE/EA/DCM = 20:1:1), white solid (135.2 mg, 99\% yield). ${ }^{1} \mathrm{H} \mathrm{NMR}\left(\mathrm{CDCl}_{3}\right.$, $400 \mathrm{MHz}): \delta$ 7.89-7.88 (m, $2 \mathrm{H}), 7.79-7.78(\mathrm{~m}, 2 \mathrm{H}), 2.77$ (t, $J=10.4 \mathrm{~Hz}, 1 \mathrm{H}), 2.12-2.09$ (m, $2 \mathrm{H})$, 1.85-1.82 (m, $2 \mathrm{H}), 1.70-1.62(\mathrm{~m}, 3 \mathrm{H}), 1.48-1.27$ (m, $3 \mathrm{H}) .{ }^{13} \mathrm{C}\left\{{ }^{1} \mathrm{H}\right\} \mathrm{NMR}\left(\mathrm{CDCl}_{3}, 100 \mathrm{MHz}\right): \delta 172.2$, $162.4,135.0,129.4,124.2,40.8,29.1,25.8,25.4$.

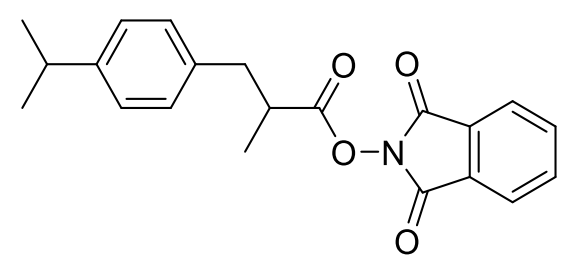

\section{1,3-Dioxoisoindolin-2-yl 3-(4-isopropylphenyl)-2-methylpropanoate (3j)}

Prepared according to the general procedure, $25^{\circ} \mathrm{C}, 12 \mathrm{~h}$. Purification was performed by flash column chromatography (PE/EA/DCM = 20:1:1), colorless oil liquid (86.1 mg, 49\% yield), m.p. 185 $187{ }^{\circ} \mathrm{C} .{ }^{1} \mathrm{H}$ NMR $\left(\mathrm{CDCl}_{3}, 400 \mathrm{MHz}\right): \delta$ 7.90-7.88 (m, $\left.2 \mathrm{H}\right), 7.80-7.78$ (m, $\left.2 \mathrm{H}\right), 7.19$ (s, $\left.4 \mathrm{H}\right), 3.28$ $3.23(\mathrm{~m}, 1 \mathrm{H}), 3.13-3.07(\mathrm{~m}, 1 \mathrm{H}), 2.94-2.87(\mathrm{~m}, 1 \mathrm{H}), 2.82-2.77(\mathrm{~m}, 1 \mathrm{H}), 1.34(\mathrm{~d}, J=6.8 \mathrm{~Hz}, 3 \mathrm{H})$, $1.26(\mathrm{~d}, J=6.8 \mathrm{~Hz}, 6 \mathrm{H}) .{ }^{13} \mathrm{C}\left\{{ }^{1} \mathrm{H}\right\} \mathrm{NMR}\left(\mathrm{CDCl}_{3}, 100 \mathrm{MHz}\right): \delta 172.6,162.3,147.6,135.5,135.1,129.4$, 129.3, 126.9, 124.3, 39.2, 39.0, 34.1, 24.3, 16.7. HRMS (ESI) $m / z[\mathrm{M}+\mathrm{Na}]^{+}$calcd. for $\mathrm{C}_{21} \mathrm{H}_{21} \mathrm{NO}_{4} \mathrm{Na}^{+}$ 374.1363, found 374.1365 .<smiles>CC(C)(C)CC(=O)ON1C(=O)c2ccccc2C1=O</smiles>

\section{1,3-Dioxoisoindolin-2-yl 3,3-dimethylbutanoate (3k) ${ }^{1}$}

Prepared according to the general procedure, $25^{\circ} \mathrm{C}, 12 \mathrm{~h}$. Purification was performed by flash column chromatography $(\mathrm{PE} / \mathrm{EA} / \mathrm{DCM}=20: 1: 1)$, white solid $(129.2 \mathrm{mg}, 99 \%$ yield $) .{ }^{1} \mathrm{H} \mathrm{NMR}\left(\mathrm{CDCl}_{3}\right.$, $400 \mathrm{MHz}): \delta$ 7.89-7.87 (m, $2 \mathrm{H}), 7.79-7.77$ (m, $2 \mathrm{H}), 2.53$ (s, $2 \mathrm{H}), 1.16(\mathrm{~s}, 9 \mathrm{H}) .{ }^{13} \mathrm{C}\left\{{ }^{1} \mathrm{H}\right\} \mathrm{NMR}\left(\mathrm{CDCl}_{3}\right.$, $100 \mathrm{MHz}): \delta$ 168.2, 162.4, 135.0, 129.4, 124.3, 44.9, 31.6, 29.8. 


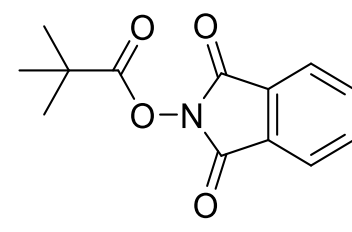

\section{1,3-Dioxoisoindolin-2-yl pivalate (3I) ${ }^{1}$}

Prepared according to the general procedure, $25{ }^{\circ} \mathrm{C}, 12 \mathrm{~h}$. Purification was performed by flash column chromatography (PE/EA/DCM $=20: 1: 1$ ), white solid (122.3 mg, 99\% yield). ${ }^{1} \mathrm{H} \mathrm{NMR}\left(\mathrm{CDCl}_{3}\right.$, $400 \mathrm{MHz}$ ): $\delta 7.89$ (dd, $J=5.2,3.2 \mathrm{~Hz}, 2 \mathrm{H}), 7.79(\mathrm{dd}, J=5.2,2.8 \mathrm{~Hz}, 2 \mathrm{H}), 1.43(\mathrm{~s}, 9 \mathrm{H}) .{ }^{13} \mathrm{C}\left\{{ }^{1} \mathrm{H}\right\} \mathrm{NMR}$ $\left(\mathrm{CDCl}_{3}, 100 \mathrm{MHz}\right): \delta 174.7,162.4,135.0,129.4,124.2,38.8,27.4$.

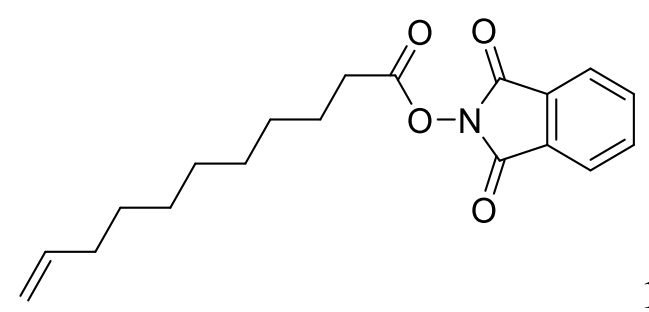

\section{1,3-Dioxoisoindolin-2-yl undec-10-enoate (3m)}

Prepared according to the general procedure, $50{ }^{\circ} \mathrm{C}, 24 \mathrm{~h}$. Purification was performed by flash column chromatography ( $\mathrm{PE} / \mathrm{EA} / \mathrm{DCM}=20: 1: 1)$, colorless oil liquid (162.9 mg, 99\% yield). ${ }^{1} \mathrm{H}$ NMR ( $\mathrm{CDCl}_{3}, 400 \mathrm{MHz}$ ): $\delta 7.89$ (dd, $\left.J=5.2,2.8 \mathrm{~Hz}, 2 \mathrm{H}\right), 7.79$ (dd, $\left.J=5.2,2.8 \mathrm{~Hz}, 2 \mathrm{H}\right), 5.86-5.76(\mathrm{~m}, 1$ H), 5.02-4.91 (m, 2 H), $2.68(\mathrm{t}, J=7.6 \mathrm{~Hz}, 2 \mathrm{H}), 2.07-2.01(\mathrm{~m}, 2 \mathrm{H}), 1.82-1.74(\mathrm{~m}, 2 \mathrm{H}), 1.44-1.32(\mathrm{~m}$, $10 \mathrm{H}) .{ }^{13} \mathrm{C}\left\{{ }^{1} \mathrm{H}\right\} \mathrm{NMR}\left(\mathrm{CDCl}_{3}, 100 \mathrm{MHz}\right): \delta 170.0,162.3,139.5,135.1,129.3,124.3,114.5,34.1,31.3$, 29.5, 29.4, 29.3, 29.2, 29.1, 25.0.<smiles>O=C(ON1C(=O)c2ccccc2C1=O)C1C=CCCC1</smiles>

\section{1,3-Dioxoisoindolin-2-yl cyclohex-2-ene-1-carboxylate (3n)}

Prepared according to the general procedure, $25{ }^{\circ} \mathrm{C}, 12 \mathrm{~h}$. Purification was performed by flash column chromatography (PE/EA/DCM = 20:1:1), white solid (61.0 mg, 45\% yield). ${ }^{1} \mathrm{H} \mathrm{NMR}\left(\mathrm{CDCl}_{3}\right.$, $400 \mathrm{MHz}$ ): $\delta 7.90$ (dd, $J=5.6,3.2 \mathrm{~Hz}, 2 \mathrm{H}$ ), 7.80 (dd, $J=5.6,3.2 \mathrm{~Hz}, 2 \mathrm{H}$ ), 5.78-5.69 (m, $2 \mathrm{H}$ ), 3.042.97 (m, $1 \mathrm{H}), 2.46-2.43(\mathrm{~m}, 2 \mathrm{H}), 2.27-2.12(\mathrm{~m}, 3 \mathrm{H}), 1.96-1.86(\mathrm{~m}, 1 \mathrm{H}) .{ }^{13} \mathrm{C}\left\{{ }^{1} \mathrm{H}\right\} \mathrm{NMR}\left(\mathrm{CDCl}_{3}, 100\right.$ MHz): $\delta$ 172.1, 162.4, 135.1, 129.3, 127.1, 124.7, 124.3, 37.2, 27.5, 25.3, 24.2. HRMS (ESI-TOF) $m / z$ $[\mathrm{M}+\mathrm{Na}]^{+}$calcd. for $\mathrm{C}_{15} \mathrm{H}_{13} \mathrm{NO}_{4} \mathrm{Na}^{+} 294.0742$, found 294.0745 .

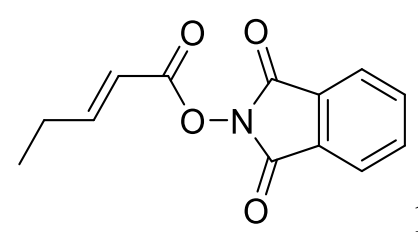

\section{1,3-Dioxoisoindolin-2-yl (E)-pent-2-enoate (3o)}

Prepared according to the general procedure, $25{ }^{\circ} \mathrm{C}, 12 \mathrm{~h}$. Purification was performed by flash column chromatography (PE/EA/DCM = 20:1:1), white solid (53.9 mg, 44\% yield), m.p. $62-63{ }^{\circ} \mathrm{C}$. ${ }^{1} \mathrm{H}$ NMR (CDCl, $\left.400 \mathrm{MHz}\right): \delta$ 7.90-7.88 (m, $\left.2 \mathrm{H}\right), 7.80-7.78$ (m, $\left.2 \mathrm{H}\right), 7.42-7.35$ (m, $\left.1 \mathrm{H}\right), 6.10$ (d, $J$ 
$=15.6 \mathrm{~Hz}, 1 \mathrm{H}), 2.39-2.33(\mathrm{~m}, 2 \mathrm{H}), 1.16(\mathrm{t}, J=7.2 \mathrm{~Hz}, 3 \mathrm{H}) \cdot{ }^{13} \mathrm{C}\left\{{ }^{1} \mathrm{H}\right\} \mathrm{NMR}\left(\mathrm{CDCl}_{3}, 100 \mathrm{MHz}\right): \delta$ $162.8,162.4,157.6,135.0,129.3,124.3,115.0,26.4,12.0$. HRMS (ESI-TOF) $\mathrm{m} / z$ [M+Na $]^{+}$calcd. for $\mathrm{C}_{13} \mathrm{H}_{11} \mathrm{NO}_{4} \mathrm{Na}^{+}$268.0586, found 268.0588.<smiles>O=C(ON1C(=O)c2ccccc2C1=O)c1ccccc1</smiles>

\section{1,3-Dioxoisoindolin-2-yl benzoate (3p) ${ }^{1}$}

Prepared according to the general procedure, $80^{\circ} \mathrm{C}, 24 \mathrm{~h}$. Purification was performed by flash column chromatography $(\mathrm{PE} / \mathrm{EA} / \mathrm{DCM}=20: 1: 1)$, white solid $(126.8 \mathrm{mg}, 95 \%$ yield $) .{ }^{1} \mathrm{H} \mathrm{NMR}\left(\mathrm{CDCl}_{3}\right.$, $400 \mathrm{MHz}): \delta 8.21(\mathrm{~d}, J=7.2 \mathrm{~Hz}, 2 \mathrm{H}), 7.95(\mathrm{dd}, J=5.6,3.2 \mathrm{~Hz}, 2 \mathrm{H}), 7.83(\mathrm{dd}, J=5.6,3.2 \mathrm{~Hz}, 2 \mathrm{H})$, $7.73(\mathrm{t}, J=7.6 \mathrm{~Hz}, 1 \mathrm{H}), 7.57(\mathrm{t}, J=8.0 \mathrm{~Hz}, 2 \mathrm{H}) .{ }^{13} \mathrm{C}\left\{{ }^{1} \mathrm{H}\right\} \mathrm{NMR}\left(\mathrm{CDCl}_{3}, 100 \mathrm{MHz}\right): \delta 163.2,162.4$, $135.2,135.1,131.0,129.4,129.2,125.6,124.4$.<smiles>Cc1ccc(C(=O)ON2C(=O)c3ccccc3C2=O)cc1</smiles>

\section{1,3-Dioxoisoindolin-2-yl 4-methylbenzoate (3q) ${ }^{1}$}

Prepared according to the general procedure, $80^{\circ} \mathrm{C}, 24 \mathrm{~h}$. Purification was performed by flash column chromatography $(\mathrm{PE} / \mathrm{EA} / \mathrm{DCM}=20: 1: 1)$, white solid $(130.7 \mathrm{mg}, 93 \%$ yield $) .{ }^{1} \mathrm{H} \mathrm{NMR}\left(\mathrm{CDCl}_{3}\right.$, $400 \mathrm{MHz}): \delta 8.09$ (d, $J=8.0 \mathrm{~Hz}, 2 \mathrm{H}), 7.93(\mathrm{dd}, J=5.2,3.2 \mathrm{~Hz}, 2 \mathrm{H}), 7.81(\mathrm{dd}, J=5.2,2.8 \mathrm{~Hz}, 2 \mathrm{H})$, $7.73(\mathrm{~d}, J=8.0 \mathrm{~Hz}, 2 \mathrm{H}), 2.46(\mathrm{~s}, 3 \mathrm{H}) .{ }^{13} \mathrm{C}\left\{{ }^{1} \mathrm{H}\right\} \mathrm{NMR}\left(\mathrm{CDCl}_{3}, 100 \mathrm{MHz}\right): \delta 163.1,162.5,146.4,135.1$, $131.0,129.9,129.4,124.3,122.8,22.2$.

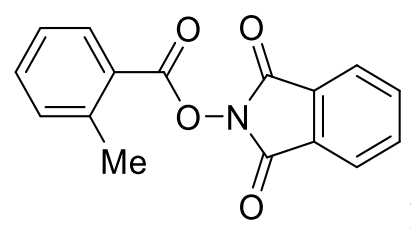

\section{1,3-Dioxoisoindolin-2-yl 2-methylbenzoate (3r) ${ }^{6}$}

Prepared according to the general procedure, $80^{\circ} \mathrm{C}, 24 \mathrm{~h}$. Purification was performed by flash column chromatography $(\mathrm{PE} / \mathrm{EA} / \mathrm{DCM}=20: 1: 1)$, white solid $(134.9 \mathrm{mg}, 96 \%$ yield $) .{ }^{1} \mathrm{H} \mathrm{NMR}\left(\mathrm{CDCl}_{3}\right.$, $400 \mathrm{MHz}$ ): $\delta 8.21$ (dd, $J=8.4,1.6 \mathrm{~Hz}, 1 \mathrm{H}), 7.94$ (dd, $J=5.6,3.2 \mathrm{~Hz}, 2 \mathrm{H}), 7.82(\mathrm{dd}, J=5.2,3.2 \mathrm{~Hz}$, $2 \mathrm{H}), 7.73(\mathrm{td}, J=7.6,1.2 \mathrm{~Hz}, 1 \mathrm{H}), 7.37$ (t, $J=7.2 \mathrm{~Hz}, 2 \mathrm{H}), 2.64(\mathrm{~s}, 2 \mathrm{H}) .{ }^{13} \mathrm{C}\left\{{ }^{1} \mathrm{H}\right\} \mathrm{NMR}\left(\mathrm{CDCl}_{3}\right.$, $100 \mathrm{MHz}): \delta$ 163.4, 162.6, 142.5, 135.1, 134.3, 132.3, 131.7, 129.4, 126.5, 124.7, 124.3, 22.0.

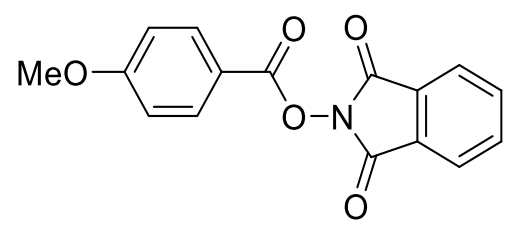

\section{1,3-Dioxoisoindolin-2-yl 4-methoxybenzoate $(3 \mathrm{~s})^{1}$}

Prepared according to the general procedure, $80{ }^{\circ} \mathrm{C}, 24 \mathrm{~h}$. Purification was performed by flash 
column chromatography (PE/EA/DCM = 20:1:1), white solid (138.1 mg, 93\% yield). ${ }^{1} \mathrm{H} \mathrm{NMR}\left(\mathrm{CDCl}_{3}\right.$, $400 \mathrm{MHz}): \delta 8.16(\mathrm{~d}, J=8.8 \mathrm{~Hz}, 2 \mathrm{H}), 7.93(\mathrm{dd}, J=5.2,3.2 \mathrm{~Hz}, 2 \mathrm{H}), 7.81-7.79(\mathrm{~m}, 2 \mathrm{H}), 7.01(\mathrm{~d}, J$ $=8.8 \mathrm{~Hz}, 2 \mathrm{H}), 3.90(\mathrm{~s}, 3 \mathrm{H}) .{ }^{13} \mathrm{C}\left\{{ }^{1} \mathrm{H}\right\} \mathrm{NMR}\left(\mathrm{CDCl}_{3}, 100 \mathrm{MHz}\right): \delta 165.2,162.7,162.6,135.1,133.3$, 129.4, 124.3, 117.6, 114.6, 55.9.

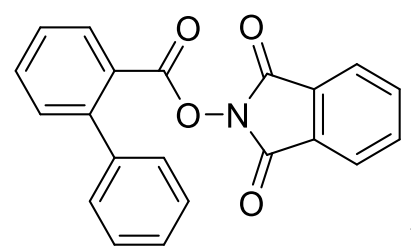

\section{1,3-Dioxoisoindolin-2-yl [1,1'-biphenyl]-2-carboxylate (3t)}

Prepared according to the general procedure, $80{ }^{\circ} \mathrm{C}, 24 \mathrm{~h}$. Purification was performed by flash column chromatography (PE/EA/DCM = 20:1:1-10:1:1), white solid (123.5 mg, 72\% yield). ${ }^{1} \mathrm{H}$ NMR $\left(\mathrm{CDCl}_{3}, 400 \mathrm{MHz}\right): \delta 8.18(\mathrm{dd}, J=7.6,0.8 \mathrm{~Hz}, 1 \mathrm{H}), 7.88(\mathrm{dd}, J=5.6,3.2 \mathrm{~Hz}, 2 \mathrm{H}), 7.77(\mathrm{dd}, J=5.6$, $3.2 \mathrm{~Hz}, 2 \mathrm{H}), 7.69$ (td, $J=7.6,1.2 \mathrm{~Hz}, 1 \mathrm{H}), 7.40-7.35(\mathrm{~m}, 7 \mathrm{H}) .{ }^{13} \mathrm{C}\left\{{ }^{1} \mathrm{H}\right\} \mathrm{NMR}\left(\mathrm{CDCl}_{3}, 100 \mathrm{MHz}\right): \delta$ 164.2, 162.3, 144.8, 140.1, 135.0, 133.6, 131.7, 131.4, 129.4, 129.1, 128.6, 128.1, 127.7, 125.4, 124.2. HRMS (ESI-TOF) $m / z[\mathrm{M}+\mathrm{Na}]^{+}$calcd. for $\mathrm{C}_{21} \mathrm{H}_{13} \mathrm{NO}_{4} \mathrm{~N}_{\mathrm{a}}{ }^{+} 366.0742$, found 366.0737.

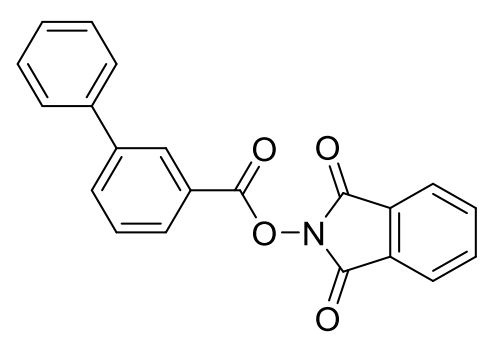

\section{1,3-Dioxoisoindolin-2-yl [1,1'-biphenyl]-3-carboxylate (3u)}

Prepared according to the general procedure, $80^{\circ} \mathrm{C}, 24 \mathrm{~h}$. Purification was performed by flash column chromatography (PE/EA/DCM = 20:1:1-10:1:1), white solid (140.7 mg, 82\% yield). ${ }^{1} \mathrm{H}$ NMR $\left(\mathrm{CDCl}_{3}, 400 \mathrm{MHz}\right): \delta 8.42(\mathrm{t}, J=1.6 \mathrm{~Hz}, 1 \mathrm{H}), 8.19$ (dt, $\left.J=7.6,1.2 \mathrm{~Hz}, 1 \mathrm{H}\right), 7.96(\mathrm{dd}, J=5.6,3.2$ Hz, $2 \mathrm{H}), 7.92-7.91(\mathrm{~m}, 1 \mathrm{H}), 7.84(\mathrm{dd}, J=5.2,2.8 \mathrm{~Hz}, 2 \mathrm{H}), 7.65-760$ (m, $3 \mathrm{H}), 7.50-7.47$ (m, $2 \mathrm{H})$, 7.43-7.39 (m, 1 H). ${ }^{13} \mathrm{C}\left\{{ }^{1} \mathrm{H}\right\} \mathrm{NMR}\left(\mathrm{CDCl}_{3}, 100 \mathrm{MHz}\right): \delta 163.2,162.4,142.5,139.8,135.2,133.9$, 129.7, 129.7, 129.6, 129.4, 128.4, 127.6, 126.2, 124.4. HRMS (ESI-TOF) $m / z[\mathrm{M}+\mathrm{Na}]^{+}$calcd. for $\mathrm{C}_{21} \mathrm{H}_{13} \mathrm{NO}_{4} \mathrm{Na}_{\mathrm{a}}{ }^{+} 366.0742$, found 366.0743 .

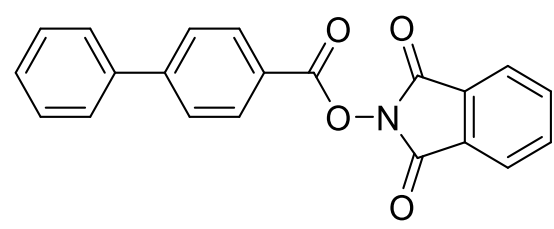

\section{1,3-Dioxoisoindolin-2-yl [1,1'-biphenyl]-4-carboxylate (3v) ${ }^{1}$}

Prepared according to the general procedure, $80^{\circ} \mathrm{C}, 24 \mathrm{~h}$. Purification was performed by flash column chromatography (PE/EA/DCM = 20:1:1-10:1:1), white solid (163.0 mg, 95\% yield). ${ }^{1} \mathrm{H}$ NMR $\left(\mathrm{CDCl}_{3}, 400 \mathrm{MHz}\right): \delta 8.28(\mathrm{~d}, J=8.4 \mathrm{~Hz}, 2 \mathrm{H}), 7.96(\mathrm{dd}, J=5.2,3.2 \mathrm{~Hz}, 2 \mathrm{H}), 7.84(\mathrm{dd}, J=5.6,3.2$ Hz, 2 H), 7.77 (d, $J=8.8$ Hz, 2 H), 7.67-7.65 (m, 2 H), 7.52-7.48 (m, 2 H), 7.46 (tt, $J=6.4,1.6 \mathrm{~Hz}, 1$ 
H). ${ }^{13} \mathrm{C}\left\{{ }^{1} \mathrm{H}\right\} \mathrm{NMR}\left(\mathrm{CDCl}_{3}, 100 \mathrm{MHz}\right): \delta 163.1,162.4,148.0,139.8,135.1,131.3,129.4,129.4,129.0$, $127.8,127.7,124.4,124.2$.<smiles>O=C(ON1C(=O)c2ccccc2C1=O)c1ccc(F)cc1</smiles>

\section{1,3-Dioxoisoindolin-2-yl 4-fluorobenzoate $(3 \mathrm{w})^{1}$}

Prepared according to the general procedure, $80^{\circ} \mathrm{C}, 24 \mathrm{~h}$. Purification was performed by flash column chromatography $(\mathrm{PE} / \mathrm{EA} / \mathrm{DCM}=20: 1: 1)$, white solid $(139.6 \mathrm{mg}, 99 \%$ yield $) .{ }^{1} \mathrm{H} \mathrm{NMR}\left(\mathrm{CDCl}_{3}\right.$, $400 \mathrm{MHz}$ ): $\delta 8.25-8.20$ (m, $2 \mathrm{H}), 7.94$ (dd, $J=5.2,2.8 \mathrm{~Hz}, 2 \mathrm{H}), 7.83$ (dd, $J=5.6,3.2 \mathrm{~Hz}, 2 \mathrm{H}), 7.24-$ $7.20(\mathrm{~m}, 2 \mathrm{H}) .{ }^{13} \mathrm{C}\left\{{ }^{1} \mathrm{H}\right\} \mathrm{NMR}\left(\mathrm{CDCl}_{3}, 100 \mathrm{MHz}\right): \delta 168.5,165.9,162.3,162.2,135.2,133.8,133.7$, $129.3,124.4,121.9,121.9,116.8,116.5$.<smiles>O=C(ON1C(=O)c2ccccc2C1=O)c1ccc(Cl)cc1</smiles>

\section{1,3-Dioxoisoindolin-2-yl 4-chlorobenzoate $(3 x)^{1}$}

Prepared according to the general procedure, $80^{\circ} \mathrm{C}, 24 \mathrm{~h}$. Purification was performed by flash column chromatography $(\mathrm{PE} / \mathrm{EA} / \mathrm{DCM}=20: 1: 1)$, white solid $(149.0 \mathrm{mg}, 99 \%$ yield $) .{ }^{1} \mathrm{H} \mathrm{NMR}\left(\mathrm{CDCl}_{3}\right.$, $400 \mathrm{MHz}): \delta 8.15(\mathrm{~d}, J=6.8 \mathrm{~Hz}, 2 \mathrm{H}), 7.94(\mathrm{dd}, J=5.2,3.2 \mathrm{~Hz}, 2 \mathrm{H}), 7.84(\mathrm{dd}, J=5.6,3.2 \mathrm{~Hz}, 2 \mathrm{H})$, $7.54(\mathrm{~d}, J=8.8 \mathrm{~Hz}, 2 \mathrm{H}) .{ }^{13} \mathrm{C}\left\{{ }^{1} \mathrm{H}\right\} \mathrm{NMR}\left(\mathrm{CDCl}_{3}, 100 \mathrm{MHz}\right): \delta 162.4,162.3,142.0,135.2,132.3,129.7$, $129.3,124.4,124.1$.<smiles>O=C(ON1C(=O)c2ccccc2C1=O)c1ccc(Br)cc1</smiles>

\section{1,3-Dioxoisoindolin-2-yl 4-bromobenzoate (3y) ${ }^{1}$}

Prepared according to the general procedure, $80{ }^{\circ} \mathrm{C}, 24 \mathrm{~h}$. Purification was performed by flash column chromatography $(\mathrm{PE} / \mathrm{EA} / \mathrm{DCM}=20: 1: 1)$, white solid $(170.8 \mathrm{mg}, 99 \%$ yield $) .{ }^{1} \mathrm{H} \mathrm{NMR}\left(\mathrm{CDCl}_{3}\right.$, $400 \mathrm{MHz}): \delta 8.06$ (d, $J=8.8 \mathrm{~Hz}, 2 \mathrm{H}), 7.94(\mathrm{dd}, J=5.2,2.8 \mathrm{~Hz}, 2 \mathrm{H}), 7.83(\mathrm{dd}, J=5.6,3.2 \mathrm{~Hz}, 2 \mathrm{H})$, $7.70(\mathrm{~d}, J=8.8 \mathrm{~Hz}, 2 \mathrm{H}) .{ }^{13} \mathrm{C}\left\{{ }^{1} \mathrm{H}\right\} \mathrm{NMR}\left(\mathrm{CDCl}_{3}, 100 \mathrm{MHz}\right): \delta 162.6,162.2,135.2,132.7,132.3,130.8$, $129.3,124.5,124.4$.

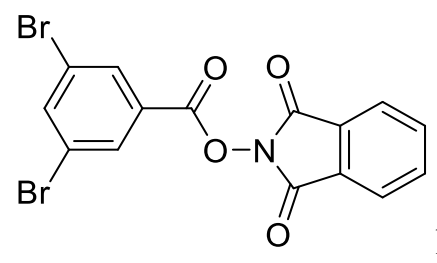

\section{1,3-Dioxoisoindolin-2-yl 3,5-dibromobenzoate (3z)}

Prepared according to the general procedure, $80{ }^{\circ} \mathrm{C}, 24 \mathrm{~h}$. Purification was performed by flash column chromatography (PE/EA/DCM = 30:1:1-10:1:1), white solid (164.9 mg, 78\% yield), m.p. 222- 
$223{ }^{\circ} \mathrm{C} .{ }^{1} \mathrm{H} \mathrm{NMR}\left(\mathrm{CDCl}_{3}, 400 \mathrm{MHz}\right): \delta 8.26(\mathrm{~d}, J=1.6 \mathrm{~Hz}, 2 \mathrm{H}), 8.00$ (t, $\left.J=1.6 \mathrm{~Hz}, 1 \mathrm{H}\right), 7.95(\mathrm{dd}, J$ $=5.6,3.2 \mathrm{~Hz}, 2 \mathrm{H}), 7.85(\mathrm{dd}, J=5.6,3.2 \mathrm{~Hz}, 2 \mathrm{H}) .{ }^{13} \mathrm{C}\left\{{ }^{1} \mathrm{H}\right\} \mathrm{NMR}\left(\mathrm{CDCl}_{3}, 100 \mathrm{MHz}\right): \delta 161.98,160.96$, $140.55,135.34,132.47,129.17,128.80,124.55,123.86$. HRMS (ESI-TOF) $m / z[\mathrm{M}+\mathrm{Na}]^{+}$calcd. for $\mathrm{C}_{15} \mathrm{H}_{7} \mathrm{Br}_{2} \mathrm{NO}_{4} \mathrm{Na}^{+}$445.8634, found 445.8637 .<smiles>O=C(ON1C(=O)c2ccccc2C1=O)c1ccc(I)cc1</smiles>

\section{1,3-Dioxoisoindolin-2-yl 4-bromobenzoate $(3 \mathrm{~A})^{1}$}

Prepared according to the general procedure, $80^{\circ} \mathrm{C}, 24 \mathrm{~h}$. Purification was performed by flash column chromatography (PE/EA/DCM = 20:1:1-10:1:1), white solid (163.1 mg, 83\% yield). ${ }^{1} \mathrm{H}$ NMR $\left(\mathrm{CDCl}_{3}, 400 \mathrm{MHz}\right): \delta 7.94-7.85(\mathrm{~m}, 6 \mathrm{H}), 7.83(\mathrm{dd}, J=5.6,3.2 \mathrm{~Hz}, 2 \mathrm{H}) .{ }^{13} \mathrm{C}\left\{{ }^{1} \mathrm{H}\right\} \mathrm{NMR}\left(\mathrm{CDCl}_{3}, 100\right.$ $\mathrm{MHz}): \delta 162.9,162.3,138.7,135.2,132.1,129.3,125.1,124.4,103.7$.<smiles>N#Cc1ccc(C(=O)ON2C(=O)c3ccccc3C2=O)cc1</smiles>

\section{1,3-Dioxoisoindolin-2-yl 4-cyanobenzoate $(3 B)^{1}$}

Prepared according to the general procedure, $80^{\circ} \mathrm{C}, 24 \mathrm{~h}$. Purification was performed by flash column chromatography $(\mathrm{PE} / \mathrm{EA} / \mathrm{DCM}=20: 1: 1)$, white solid $(138.7 \mathrm{mg}, 95 \%$ yield $) .{ }^{1} \mathrm{H} \mathrm{NMR}\left(\mathrm{CDCl}_{3}\right.$, $400 \mathrm{MHz}): \delta 8.32(\mathrm{~d}, J=8.8 \mathrm{~Hz}, 2 \mathrm{H}), 7.96(\mathrm{dd}, J=5.6,3.2 \mathrm{~Hz}, 2 \mathrm{H}), 7.87-7.82(\mathrm{~m}, 4 \mathrm{H}) .{ }^{13} \mathrm{C}\left\{{ }^{1} \mathrm{H}\right\} \mathrm{NMR}$ $\left(\mathrm{CDCl}_{3}, 100 \mathrm{MHz}\right): \delta 162.0,161.8,135.4,133.0,131.4,129.5,129.2,124.6,118.6,117.8$.

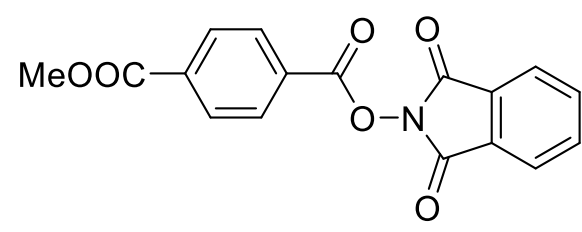

\section{1,3-Dioxoisoindolin-2-yl methyl terephthalate (3C) ${ }^{1}$}

Prepared according to the general procedure, $80{ }^{\circ} \mathrm{C}, 24 \mathrm{~h}$. Purification was performed by flash column chromatography (PE/EA/DCM =30:1:1-10:1:1), white solid (147.9 mg, 91\% yield). ${ }^{1} \mathrm{H}$ NMR $\left(\mathrm{CDCl}_{3}, 400 \mathrm{MHz}\right): \delta 8.28(\mathrm{~d}, J=8.4 \mathrm{~Hz}, 2 \mathrm{H}), 8.21(\mathrm{~d}, J=8.4 \mathrm{~Hz}, 2 \mathrm{H}), 7.95(\mathrm{dd}, J=5.6,3.2 \mathrm{~Hz}, 2$ $\mathrm{H}), 7.84(\mathrm{dd}, J=5.6,3.2 \mathrm{~Hz}, 2 \mathrm{H}) .{ }^{13} \mathrm{C}\left\{{ }^{1} \mathrm{H}\right\} \mathrm{NMR}\left(\mathrm{CDCl}_{3}, 100 \mathrm{MHz}\right): \delta 166.13,162.50,162.19,135.91$, $135.24,130.94,130.24,129.33,129.24,124.45,53.00$.

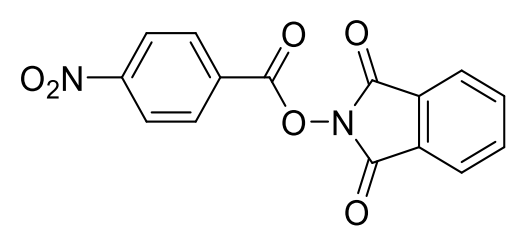

\section{1,3-Dioxoisoindolin-2-yl 4-nitrobenzoate (3D) ${ }^{1}$}

Prepared according to the general procedure, $80^{\circ} \mathrm{C}, 24 \mathrm{~h}$. Purification was performed by flash column chromatography (PE/EA/DCM = 20:1:1), white solid (88.9 mg, 57\% yield). ${ }^{1} \mathrm{H} \mathrm{NMR}\left(\mathrm{CDCl}_{3}\right.$, 
$400 \mathrm{MHz}): \delta 8.40(\mathrm{~s}, 4 \mathrm{H}), 7.97(\mathrm{dd}, J=5.6,2.8 \mathrm{~Hz}, 2 \mathrm{H}), 7.86(\mathrm{dd}, J=5.6,3.2 \mathrm{~Hz}, 2 \mathrm{H}) .{ }^{13} \mathrm{C}\left\{{ }^{1} \mathrm{H}\right\} \mathrm{NMR}$ $\left(\mathrm{CDCl}_{3}, 100 \mathrm{MHz}\right): \delta 162.0,161.6,151.9,135.4,132.2,131.1,129.2,124.6,124.3$.<smiles>O=C(ON1C(=O)c2ccccc2C1=O)c1cccc2ccccc12</smiles>

\section{1,3-Dioxoisoindolin-2-yl 1-naphthoate (3E) ${ }^{1}$}

Prepared according to the general procedure, $80{ }^{\circ} \mathrm{C}, 24 \mathrm{~h}$. Purification was performed by flash column chromatography (PE/EA/DCM = 20:1:1-10:1:1), white solid (118.9 mg, 75\% yield). ${ }^{1} \mathrm{H}$ NMR $\left(\mathrm{CDCl}_{3}, 400 \mathrm{MHz}\right): \delta 8.87(\mathrm{~d}, J=8.4 \mathrm{~Hz}, 1 \mathrm{H}), 8.56(\mathrm{dd}, J=7.2,0.8 \mathrm{~Hz}, 1 \mathrm{H}), 8.19(\mathrm{~d}, J=8.0 \mathrm{~Hz}, 1$ H), 7.98-7.93 (m, $3 \mathrm{H}), 7.85$ (dd, $J=5.6,3.2 \mathrm{~Hz}, 2 \mathrm{H}), 7.69-7.65(\mathrm{~m}, 1 \mathrm{H}), 7.63-7.58(\mathrm{~m}, 2 \mathrm{H})$. ${ }^{13} \mathrm{C}\left\{{ }^{1} \mathrm{H}\right\} \mathrm{NMR}\left(\mathrm{CDCl}_{3}, 100 \mathrm{MHz}\right): \delta 163.4,162.7,135.9,135.2,134.1,132.3,131.9,129.4,129.1$, $129.1,127.2,125.7,124.9,124.4,122.0$.<smiles>O=C(ON1C(=O)c2ccccc2C1=O)c1ccc2ccccc2c1</smiles>

\section{1,3-Dioxoisoindolin-2-yl 2-naphthoate (3F) ${ }^{1}$}

Prepared according to the general procedure, $80^{\circ} \mathrm{C}, 24 \mathrm{~h}$. Purification was performed by flash column chromatography (PE/EA/DCM = 20:1:1-10:1:1), white solid (137.9 mg, 87\% yield). ${ }^{1} \mathrm{H}$ NMR $\left(\mathrm{CDCl}_{3}, 400 \mathrm{MHz}\right): \delta 8.83(\mathrm{~s}, 1 \mathrm{H}), 8.16(\mathrm{dd}, J=8.8,2.0 \mathrm{~Hz}, 1 \mathrm{H}), 8.02-7.92(\mathrm{~m}, 5 \mathrm{H}), 7.84(\mathrm{dd}, J=$ 5.2, $3.2 \mathrm{~Hz}, 2 \mathrm{H}), 7.70(\mathrm{dt}, J=6.8,1.2 \mathrm{~Hz}, 2 \mathrm{H}) .{ }^{13} \mathrm{C}\left\{{ }^{1} \mathrm{H}\right\} \mathrm{NMR}\left(\mathrm{CDCl}_{3}, 100 \mathrm{MHz}\right): \delta 163.3,162.5$, $136.6,135.2,133.4,132.6,130.0,129.7,129.4,129.2,128.3,127.6,125.5,124.4,122.7$.<smiles>O=C(ON1C(=O)c2ccccc2C1=O)c1cccs1</smiles>

\section{1,3-Dioxoisoindolin-2-yl thiophene-2-carboxylate (3G)}

Prepared according to the general procedure, $80^{\circ} \mathrm{C}, 24 \mathrm{~h}$. Purification was performed by flash column chromatography (PE/EA/DCM =10:1:1), white solid (95.6 mg, 70\% yield). ${ }^{1} \mathrm{H} \mathrm{NMR}\left(\mathrm{CDCl}_{3}\right.$, $400 \mathrm{MHz}): \delta 8.09$ (d, $J=4.0 \mathrm{~Hz}, 1 \mathrm{H}), 7.94(\mathrm{dd}, J=5.2,2.8 \mathrm{~Hz}, 2 \mathrm{H}), 7.83-7.92(\mathrm{~m}, 3 \mathrm{H}), 7.24(\mathrm{t}, J=$ $4.8 \mathrm{~Hz}, 1 \mathrm{H}) .{ }^{13} \mathrm{C}\left\{{ }^{1} \mathrm{H}\right\} \mathrm{NMR}\left(\mathrm{CDCl}_{3}, 100 \mathrm{MHz}\right): 162.3,158.6,137.0,134.0,135.2,129.3,128.7,127.4$, 124.4.<smiles>O=C(ON1C(=O)c2ccccc2C1=O)c1ccsc1</smiles>

\section{1,3-Dioxoisoindolin-2-yl thiophene-3-carboxylate $(3 \mathrm{H})^{6}$}

Prepared according to the general procedure, $80{ }^{\circ} \mathrm{C}, 24 \mathrm{~h}$. Purification was performed by flash column chromatography $(\mathrm{PE} / \mathrm{EA} / \mathrm{DCM}=10: 1: 1)$, white solid $(117.4 \mathrm{mg}, 86 \%$ yield $) .{ }^{1} \mathrm{H} \mathrm{NMR}\left(\mathrm{CDCl}_{3}\right.$, 
$400 \mathrm{MHz}): \delta 8.45(\mathrm{dd}, J=2.8,0.8 \mathrm{~Hz}, 1 \mathrm{H}), 7.94(\mathrm{dd}, J=5.6,3.2 \mathrm{~Hz}, 2 \mathrm{H}), 7.83(\mathrm{dd}, J=5.6,3.2 \mathrm{~Hz}$, $2 \mathrm{H}), 7.68(\mathrm{dd}, J=5.2,1.2 \mathrm{~Hz}, 1 \mathrm{H}), 7.44(\mathrm{dd}, J=4.8,2.8 \mathrm{~Hz}, 1 \mathrm{H}) .{ }^{13} \mathrm{C}\left\{{ }^{1} \mathrm{H}\right\} \mathrm{NMR}\left(\mathrm{CDCl}_{3}, 100 \mathrm{MHz}\right)$ : $162.4,158.8,136.5,135.2,129.3,128.5,127.8,127.4,124.4$.<smiles>O=C(ON1C(=O)c2ccccc2C1=O)c1ccco1</smiles>

\section{1,3-Dioxoisoindolin-2-yl furan-2-carboxylate (3J) ${ }^{1}$}

Prepared according to the general procedure, $80^{\circ} \mathrm{C}, 24 \mathrm{~h}$. Purification was performed by flash column chromatography (PE/EA/DCM =10:1:1), white solid (41.1 mg, 32\% yield). ${ }^{1} \mathrm{H} \mathrm{NMR}\left(\mathrm{CDCl}_{3}\right.$, $400 \mathrm{MHz}): \delta 7.93(\mathrm{dd}, J=5.6,3.2 \mathrm{~Hz}, 2 \mathrm{H}), 7.83(\mathrm{dd}, J=5.2,2.8 \mathrm{~Hz}, 2 \mathrm{H}), 7.76(\mathrm{~d}, J=0.8 \mathrm{~Hz}, 1 \mathrm{H})$, $7.54(\mathrm{~d}, J=3.6 \mathrm{~Hz}, 1 \mathrm{H}), 6.66(\mathrm{t}, J=3.6,1.6 \mathrm{~Hz}, 1 \mathrm{H}) .{ }^{13} \mathrm{C}\left\{{ }^{1} \mathrm{H}\right\} \mathrm{NMR}\left(\mathrm{CDCl}_{3}, 100 \mathrm{MHz}\right): 162.2,154.8$, $149.1,140.2,135.2,129.2,124.4,122.6,113.0$.<smiles>O=C(ON1C(=O)c2ccccc2C1=O)c1cccnc1</smiles>

\section{1,3-dioxoisoindolin-2-yl nicotinate $(3 \mathrm{~K})^{6}$}

Prepared according to the general procedure, $80^{\circ} \mathrm{C}, 24 \mathrm{~h}$. Purification was performed by flash column chromatography $(\mathrm{PE} / \mathrm{EA} / \mathrm{DCM}=10: 1: 1)$, white solid $(132.7 \mathrm{mg}, 99 \%$ yield $) .{ }^{1} \mathrm{H} \mathrm{NMR}\left(\mathrm{CDCl}_{3}\right.$, $400 \mathrm{MHz}): \delta 9.39$ (s, $1 \mathrm{H}), 8.92(\mathrm{~d}, J=4.5 \mathrm{~Hz}, 1 \mathrm{H}), 8.45$ (d, $J=8.0 \mathrm{~Hz}, 2 \mathrm{H}), 7.94$ (dd, $J=5.2,3.1$ $\mathrm{Hz}, 2 \mathrm{H}), 7.83(\mathrm{dd}, J=5.3,3.1 \mathrm{~Hz}, 2 \mathrm{H}), 7.51(\mathrm{dd}, J=7.8,4.9 \mathrm{~Hz}, 2 \mathrm{H}) .{ }^{13} \mathrm{C}\left\{{ }^{1} \mathrm{H}\right\} \mathrm{NMR}\left(\mathrm{CDCl}_{3}, 100\right.$ $\mathrm{MHz}): \delta 162.1,162.1,155.5,151.9,138.3,135.3,129.2,124.5,124.0,122.2$.

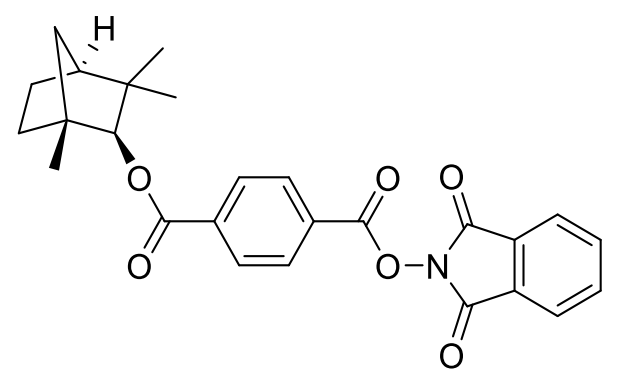

\section{1,3-Dioxoisoindolin-2-yl $\quad((1 \mathrm{R}, 2 \mathrm{R}, 4 \mathrm{~S})-1,3,3-$ trimethylbicyclo[2.2.1]heptan-2-yl) $\quad$ terephthalate} (3L)

Prepared according to the general procedure, $80{ }^{\circ} \mathrm{C}, 24 \mathrm{~h}$. Purification was performed by flash column chromatography (PE/EA/DCM = 20:1:1), white solid (154.3 mg, 69\% yield), m.p. 126-127 ${ }^{\circ} \mathrm{C} .{ }^{1} \mathrm{H} \mathrm{NMR}\left(\mathrm{CDCl}_{3}, 400 \mathrm{MHz}\right): \delta 8.29(\mathrm{~d}, J=8.8 \mathrm{~Hz}, 2 \mathrm{H}), 8.21(\mathrm{~d}, J=8.8 \mathrm{~Hz}, 2 \mathrm{H}), 7.95(\mathrm{dd}, J=$ 5.2, 3.2 Hz, 2 H), $7.84(\mathrm{dd}, J=5.2,2.8 \mathrm{~Hz}, 2 \mathrm{H}), 4.66$ (d, $J=2.0 \mathrm{~Hz}, 1 \mathrm{H}), 1.96-1.88$ (m, $1 \mathrm{H}), 1.82-$ 1.77 (m, 2 H), 1.69-1.63 (m, 1 H), 1.58-1.49 (m, 1 H), 1.29-1.23 (m, 2 H), 1.20 (s, 3 H), 1.13 (s, 3 H), 0.85 (s, $3 \mathrm{H}) .{ }^{13} \mathrm{C}\left\{{ }^{1} \mathrm{H}\right\} \mathrm{NMR}\left(\mathrm{CDCl}_{3}, 100 \mathrm{MHz}\right): \delta 166.0,162.6,162.2,136.5,135.2,131.0,130.2$, 129.3, 129.2, 124.4, 87.9, 49.0, 48.7, 41.8, 40.2, 30.1, 27.2, 26.2, 20.6, 19.8. HRMS (ESI-TOF) $\mathrm{m} / z$ 


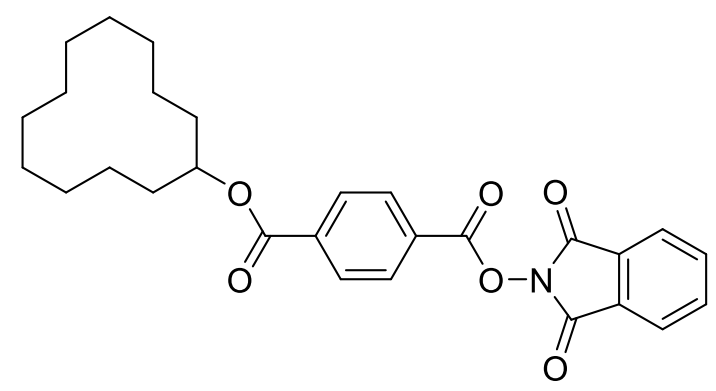

\section{1,3-Dioxoisoindolin-2-yl 4-(cyclododecyloxy)benzoate (3M)}

Prepared according to the general procedure, $80^{\circ} \mathrm{C}, 24 \mathrm{~h}$. Purification was performed by flash column chromatography $(\mathrm{PE} / \mathrm{EA} / \mathrm{DCM}=20: 1: 1)$, white solid (164.6 mg, 69\% yield), m.p. 132-133 ${ }^{\circ} \mathrm{C} .{ }^{1} \mathrm{H} \mathrm{NMR}\left(\mathrm{CDCl}_{3}, 400 \mathrm{MHz}\right): \delta 8.26(\mathrm{~d}, J=8.4 \mathrm{~Hz}, 2 \mathrm{H}), 8.19(\mathrm{~d}, J=8.8 \mathrm{~Hz}, 2 \mathrm{H}), 7.95(\mathrm{dd}, J=$ 5.2, $2.8 \mathrm{~Hz}, 2 \mathrm{H}), 7.84$ (dd, $J=5.6,3.2 \mathrm{~Hz}, 2 \mathrm{H}), 5.33-5.27$ (m, $1 \mathrm{H}), 1.91-1.83$ (m, $2 \mathrm{H}), 1.70-1.64$ (m, $2 \mathrm{H}), 1.48-1.25(\mathrm{~m}, 19 \mathrm{H}) .{ }^{13} \mathrm{C}\left\{{ }^{1} \mathrm{H}\right\} \mathrm{NMR}\left(\mathrm{CDCl}_{3}, 100 \mathrm{MHz}\right): \delta 165.3,162.6,162.2,136.8,135.2$, $130.9,130.2$, 129.3, 129.1, 124.5, 74.4, 29.4, 24.5, 24.3, 23.6, 23.4, 21.2. HRMS (ESI-TOF) $\mathrm{m} / z$ $[\mathrm{M}+\mathrm{Na}]^{+}$calcd. for $\mathrm{C}_{28} \mathrm{H}_{31} \mathrm{NO}_{6} \mathrm{Na}^{+}$500.2044, found 500.2046.<smiles>CCOC(=O)CCCCC(=O)ON1C(=O)c2ccccc2C1=O</smiles>

\section{1,3-dioxoisoindolin-2-yl ethyl adipate $(3 \mathrm{~N})^{7}$}

Prepared according to the general procedure, $50{ }^{\circ} \mathrm{C}, 12 \mathrm{~h}$. Purification was performed by flash column chromatography $(\mathrm{PE} / \mathrm{EA} / \mathrm{DCM}=10: 1: 1)$, white solid $(154.8 \mathrm{mg}, 97 \%) .{ }^{1} \mathrm{H} \mathrm{NMR}(400 \mathrm{MHz}$, $\left.\mathrm{CDCl}_{3}\right) \delta 7.90(\mathrm{dd}, J=3.2,5.6 \mathrm{~Hz}, 2 \mathrm{H}), 7.80(\mathrm{dd}, J=2.8,5.2 \mathrm{~Hz}, 2 \mathrm{H}), 4.17(\mathrm{q}, J=7.2 \mathrm{~Hz}, 2 \mathrm{H}), 2.72$ $(\mathrm{t}, J=6.8 \mathrm{~Hz}, 2 \mathrm{H}), 2.39(\mathrm{t}, J=7.2 \mathrm{~Hz}, 2 \mathrm{H}), 1.87-1.74(\mathrm{~m}, 4 \mathrm{H}), 1.28(\mathrm{t}, J=7.2 \mathrm{~Hz}, 3 \mathrm{H}) .{ }^{13} \mathrm{C}\left\{{ }^{1} \mathrm{H}\right\} \mathrm{NMR}$ $\left(100 \mathrm{MHz}, \mathrm{CDCl}_{3}\right) \delta 173.4,169.6,162.3,135.1,129.3,124.3,60.8,34.1,31.0,24.4,14.6$.<smiles>O=C(CCCCCOC(=O)c1ccc(Cl)cc1)ON1C(=O)c2ccccc2C1=O</smiles>

\section{6-((1,3-dioxoisoindolin-2-yl)oxy)-6-oxohexyl 4-chlorobenzoate (30)}

Prepared according to the general procedure, $50{ }^{\circ} \mathrm{C}, 12 \mathrm{~h}$. Purification was performed by flash column chromatography (PE/EA/DCM =10:1:1), colorless oil liquid (199.2 mg, 96\%). ${ }^{1} \mathrm{H}$ NMR (400 $\left.\mathrm{MHz}, \mathrm{CDCl}_{3}\right) \delta 7.99(\mathrm{~d}, J=8.4 \mathrm{~Hz}, 2 \mathrm{H}), 7.88(\mathrm{dd}, J=3.2,5.6 \mathrm{~Hz}, 2 \mathrm{H}), 7.80(\mathrm{dd}, J=2.8,5.2 \mathrm{~Hz}, 2$ H), $7.41(\mathrm{~d}, J=8.8 \mathrm{~Hz}, 2 \mathrm{H}), 4.36(\mathrm{t}, J=6.8 \mathrm{~Hz}, 2 \mathrm{H}), 2.73(\mathrm{t}, J=7.6 \mathrm{~Hz}, 2 \mathrm{H}), 1.91-1.80(\mathrm{~m}, 4 \mathrm{H})$, 1.66-1.58 (m, $2 \mathrm{H}) .{ }^{13} \mathrm{C}\left\{{ }^{1} \mathrm{H}\right\} \mathrm{NMR}\left(100 \mathrm{MHz}, \mathrm{CDCl}_{3}\right) \delta 169.7,166.1,162.3,139.6,135.1,131.3,129.2$, 129.1, 129.0, 124.3, 65.1, 31.2, 28.6, 25.6, 24.7. HRMS (ESI-TOF) $m / z[\mathrm{M}+\mathrm{Na}]^{+}$calcd. for 


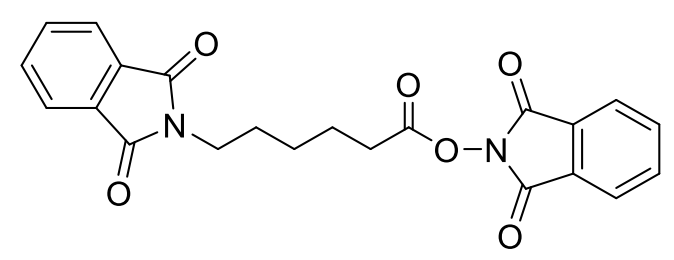

\section{1,3-dioxoisoindolin-2-yl 6-(1,3-dioxoisoindolin-2-yl)hexanoate (3P)}

Prepared according to the general procedure, $50{ }^{\circ} \mathrm{C}, 12 \mathrm{~h}$. Purification was performed by flash column chromatography $(\mathrm{PE} / \mathrm{EA} / \mathrm{DCM}=10: 1: 1)$, white solid $(198.9 \mathrm{mg}, 98 \%) .{ }^{1} \mathrm{H} \mathrm{NMR}(400 \mathrm{MHz}$, $\left.\mathrm{CDCl}_{3}\right) \delta 7.88-7.83(\mathrm{~m}, 4 \mathrm{H}), 7.79(\mathrm{dd}, J=2.8,5.6 \mathrm{~Hz}, 2 \mathrm{H}), 7.72(\mathrm{dd}, J=2.8,5.2 \mathrm{~Hz}, 2 \mathrm{H}), 3.74(\mathrm{t}, J$ $=7.2 \mathrm{~Hz}, 2 \mathrm{H}), 2.69$ (t, $J=7.6 \mathrm{~Hz}, 2 \mathrm{H}), 1.88-1.81(\mathrm{~m}, 2 \mathrm{H}), 1.79-1.72(\mathrm{~m}, 2 \mathrm{H}), 1.55-1.47$ (m, $2 \mathrm{H})$. ${ }^{13} \mathrm{C}\left\{{ }^{1} \mathrm{H}\right\} \mathrm{NMR}\left(100 \mathrm{MHz}, \mathrm{CDCl}_{3}\right) \delta 169.7,168.8,162.2,135.1,134.2,132.5,129.3,124.3,123.6,37.9$, 31.2, 28.5, 26.3, 24.6. HRMS (ESI-TOF) $m / z$ [M+Na] $]^{+}$calcd. for $\mathrm{C}_{22} \mathrm{H}_{18} \mathrm{~N}_{2} \mathrm{O}_{6} \mathrm{Na} 429.1063$, found 429.1059 .

\subsection{General Procedure for Preparation of $N$-Hydroxyimide Esters from Aldehydes}

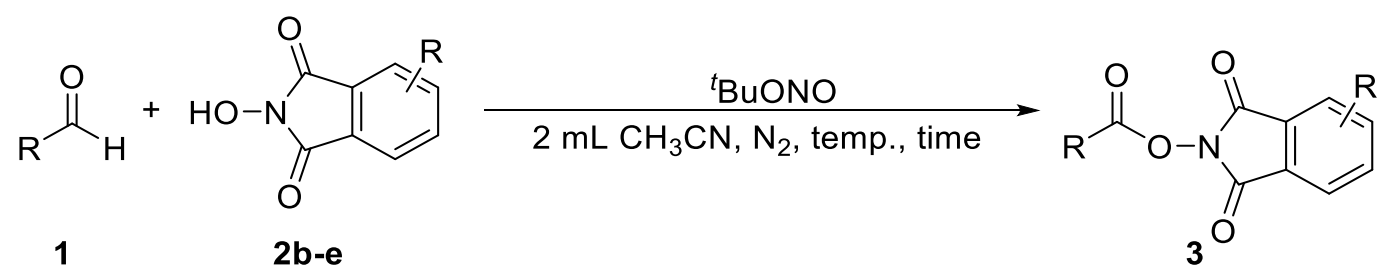

$N$-hydroxyimide ( 0.25 or $0.5 \mathrm{mmol}, 1.0$ equiv) was weighed directly into a Schlenk tube equipped with a magnetic stir bar. After dried in vacuo for $15 \mathrm{~min}$ and charged with nitrogen gas, dry MeCN (2 $\mathrm{mL}$ ) was added. Then aldehyde ( 0.375 or $0.75 \mathrm{mmol}, 1.5$ equiv) was added dropwise into the mixture. ${ }^{t} \mathrm{BuONO}$ (TBN) (30 or $60 \mu \mathrm{L}, 0.25$ or $0.5 \mathrm{mmol}, 1.0$ equiv) was last added and the tube was capped with a glass cap. The resulting solution was stirred at 50 or $80{ }^{\circ} \mathrm{C}$ for 12 or $24 \mathrm{~h}$. After cooling to rt, the reaction mixture was diluted with $\mathrm{CH}_{2} \mathrm{Cl}_{2}(2 \mathrm{~mL})$, the solvent was removed under vacuum and the residue was purified by column chromatography on silica gel to give the pure product.<smiles>O=C(ON1C(=O)c2c(Cl)c(Cl)c(Cl)c(Cl)c2C1=O)C1CCCCC1</smiles>

\section{4,5,6,7-Tetrachloro-1,3-dioxoisoindolin-2-yl cyclohexanecarboxylate $(3 Q)^{8}$}

Prepared according to the general procedure, $50{ }^{\circ} \mathrm{C}, 12 \mathrm{~h}$. Purification was performed by flash column chromatography $(\mathrm{PE} / \mathrm{EA} / \mathrm{DCM}=20: 1: 1)$, white solid $(173.80 \mathrm{mg}, 82 \%) .{ }^{1} \mathrm{H} \mathrm{NMR}(400 \mathrm{MHz}$, $\left.\mathrm{CDCl}_{3}\right) \delta$ 2.77-2.70 (m, $\left.1 \mathrm{H}\right), 2.09-2.05$ (m, $\left.2 \mathrm{H}\right), 1.85-1.81$ (m, $\left.2 \mathrm{H}\right), 1.69-1.63$ (m, $\left.3 \mathrm{H}\right), 1.44-1.25$ $(\mathrm{m}, 3 \mathrm{H}) .{ }^{13} \mathrm{C}\left\{{ }^{1} \mathrm{H}\right\} \mathrm{NMR}\left(100 \mathrm{MHz}, \mathrm{CDCl}_{3}\right) \delta 171.5,157.8,141.1,130.5,124.9,40.5,28.9,25.5,25.1$. 


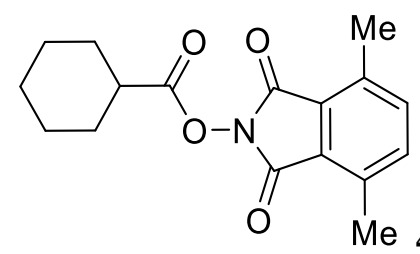

4,7-Dimethyl-1,3-dioxoisoindolin-2-yl cyclohexanecarboxylate (3R)

Prepared according to the general procedure, $50{ }^{\circ} \mathrm{C}, 12 \mathrm{~h}$. Purification was performed by flash column chromatography $(\mathrm{PE} / \mathrm{EA} / \mathrm{DCM}=20: 1: 1)$, white solid $(144.54 \mathrm{mg}, 96 \%) .{ }^{1} \mathbf{H}$ NMR $(400 \mathrm{MHz}$, $\left.\mathrm{CDCl}_{3}\right) \delta 7.36(\mathrm{~s}, 2 \mathrm{H}), 2.78-2.69(\mathrm{~m}, 1 \mathrm{H}), 2.62(\mathrm{~s}, 6 \mathrm{H}), 2.11-2.07(\mathrm{~m}, 2 \mathrm{H}), 1.86-1.81(\mathrm{~m}, 2 \mathrm{H}), 1.70-$ $1.64(\mathrm{~m}, 3 \mathrm{H}), 1.43-1.25(\mathrm{~m}, 3 \mathrm{H}) .{ }^{13} \mathrm{C}\left\{{ }^{1} \mathrm{H}\right\} \mathrm{NMR}\left(100 \mathrm{MHz}, \mathrm{CDCl}_{3}\right) \delta 172.2,163.1,136.8,136.3$, 125.9, 40.6, 28.9, 25.6, 25.1, 17.5. HRMS (ESI-TOF) $\mathrm{m} / z[\mathrm{M}+\mathrm{H}]^{+}$calcd. for $\mathrm{C}_{17} \mathrm{H}_{20} \mathrm{NO}_{4}{ }^{+} 302.1392$, found 302.1392 .

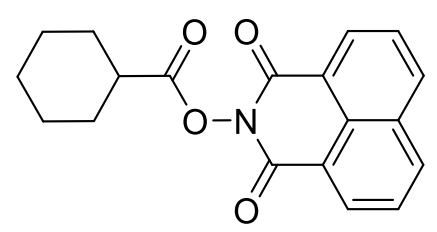

\section{1,3-Dioxo-1 H-benzo[de]isoquinolin-2(3H)-yl cyclohexanecarboxylate (3S)}

Prepared according to the general procedure, $0.25 \mathrm{mmol}, 2.0$ equiv TBN, $80{ }^{\circ} \mathrm{C}, 24 \mathrm{~h}$. Purification was performed by flash column chromatography (PE/EA/DCM = 20:1:1), white solid (58.16 mg, 72\%). ${ }^{1} \mathrm{H}$ NMR (400 MHz, $\left.\mathrm{CDCl}_{3}\right) \delta 8.62(\mathrm{dd}, J=7.2,0.8 \mathrm{~Hz}, 2 \mathrm{H}), 8.28(\mathrm{dd}, J=8.4,0.8 \mathrm{~Hz}, 2 \mathrm{H}), 7.80$ (t, $J=7.6 \mathrm{~Hz}, 2 \mathrm{H}), 2.88-2.81$ (m, 1 H), 2.21-2.17 (m, 2 H), 1.90-1.85 (m, 2 H), 1.78-1.68 (m, 3 H), 1.47$1.25(\mathrm{~m}, 3 \mathrm{H}) .{ }^{13} \mathrm{C}\left\{{ }^{1} \mathrm{H}\right\} \mathrm{NMR}\left(100 \mathrm{MHz}, \mathrm{CDCl}_{3}\right) \delta 172.0,159.8,135.1,132.0,132.0,127.8,127.2$, $122.5,40.9,29.0,29.0,25.7,25.5,25.3$. HRMS (ESI-TOF) $m / z[\mathrm{M}+\mathrm{Na}]^{+}$calcd. for $\mathrm{C}_{19} \mathrm{H}_{17} \mathrm{NO}_{4} \mathrm{Na}^{+}$ 346.1055 , found 346.1057 .

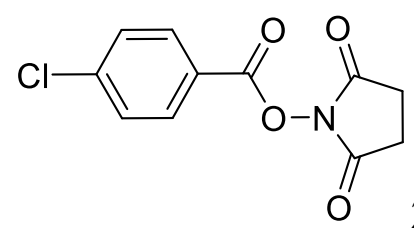

\section{2,5-Dioxopyrrolidin-1-yl 4-chlorobenzoate (3T)}

Prepared according to the general procedure, $80^{\circ} \mathrm{C}, 24 \mathrm{~h}$. Purification was performed by flash column chromatography $(\mathrm{PE} / \mathrm{EA} / \mathrm{DCM}=20: 1: 1)$, white solid $(107.53 \mathrm{mg}, 85 \%) .{ }^{1} \mathrm{H} \mathrm{NMR}(400 \mathrm{MHz}$, $\left.\mathrm{CDCl}_{3}\right) \delta 8.09(\mathrm{~d}, J=8.8 \mathrm{~Hz}, 2 \mathrm{H}), 7.51(\mathrm{~d}, J=8.8 \mathrm{~Hz}, 2 \mathrm{H}), 2.91(\mathrm{~s}, 4 \mathrm{H}) .{ }^{13} \mathrm{C}\left\{{ }^{1} \mathrm{H}\right\} \mathrm{NMR}(100 \mathrm{MHz}$, $\left.\mathrm{CDCl}_{3}\right) \delta 169.2,161.3,141.9,132.0,129.5,123.7,25.8$.

\subsection{General Procedure for Preparation of $\mathrm{N}$-Hydroxyimide Esters from 2-Oxo-2- phenylacetaldehyde}




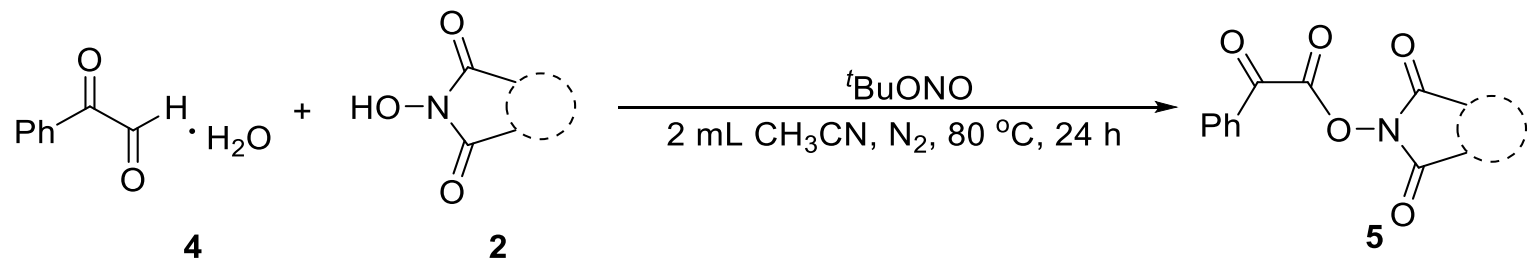

$N$-hydroxyimide ( 0.25 or $0.5 \mathrm{mmol}, 1.0$ equiv) was weighed directly into a Schlenk tube equipped with a magnetic stir bar. After dried in vacuo for 15 min and charged with nitrogen gas, dry MeCN (2 $\mathrm{mL}$ ) was added. Then aldehyde ( 0.375 or $0.75 \mathrm{mmol}, 1.5$ equiv) was added dropwise into the mixture. ${ }^{t} \mathrm{BuONO}(\mathrm{TBN})(60$ or $120 \mu \mathrm{L}, 0.5$ or $1.0 \mathrm{mmol}, 2.0$ equiv) was last added and the tube was capped with a glass cap. The resulting solution was stirred at $80^{\circ} \mathrm{C}$ for $24 \mathrm{~h}$. After cooling to rt, the reaction mixture was diluted with $\mathrm{CH}_{2} \mathrm{Cl}_{2}(2 \mathrm{~mL})$, the solvent was removed under vacuum and the residue was purified by column chromatography on silica gel to give the pure product.<smiles>O=C(ON1C(=O)c2ccccc2C1=O)C(=O)c1ccccc1</smiles>

\section{1,3-Dioxoisoindolin-2-yl 2-oxo-2-phenylacetate (5a)}

Prepared according to the general procedure, $80^{\circ} \mathrm{C}, 24 \mathrm{~h}$. Purification was performed by flash column chromatography (PE/EA/DCM = 20:1:1-10:1:1), white solid (76.8 mg, 52\% yield), m.p. 157$158{ }^{\circ} \mathrm{C} .{ }^{1} \mathrm{H}$ NMR $\left(\mathrm{CDCl}_{3}, 500 \mathrm{MHz}\right): \delta 8.19(\mathrm{~d}, J=7.4 \mathrm{~Hz}, 2 \mathrm{H}), 7.92(\mathrm{dd}, J=5.4,3.2 \mathrm{~Hz}, 2 \mathrm{H}), 7.81$ $(\mathrm{dd}, J=5.4,3.2 \mathrm{~Hz}, 2 \mathrm{H}), 7.70(\mathrm{t}, J=7.5 \mathrm{~Hz}, 1 \mathrm{H}), 7.54(\mathrm{t}, J=7.9 \mathrm{~Hz}, 2 \mathrm{H}) .{ }^{13} \mathrm{C}\left\{{ }^{1} \mathrm{H}\right\} \mathrm{NMR}\left(\mathrm{CDCl}_{3}\right.$, $125 \mathrm{MHz}): \delta 163.2,162.4,135.2,135.1,131.0,129.4,129.2,125.6,124.4$. HRMS (ESI-TOF) $\mathrm{m} / z$ $[\mathrm{M}+\mathrm{Na}]^{+}$calcd. for $\mathrm{C}_{16} \mathrm{H}_{9} \mathrm{NO}_{5} \mathrm{Na}^{+} 318.0373$, found 318.0376 .<smiles>Cc1ccc(C)c2c1C(=O)N(OC(=O)C(=O)c1ccccc1)C2=O</smiles>

\section{4,7-Dimethyl-1,3-dioxoisoindolin-2-yl 2-oxo-2-phenylacetate (5b)}

Prepared according to the general procedure, $80^{\circ} \mathrm{C}, 24 \mathrm{~h}$. Purification was performed by flash column chromatography $(\mathrm{PE} / \mathrm{EA} / \mathrm{DCM}=20: 1: 1)$, white solid $(96.92 \mathrm{mg}, 60 \%) .{ }^{1} \mathrm{H} \mathrm{NMR}(400 \mathrm{MHz}$, $\left.\mathrm{CDCl}_{3}\right) \delta 8.21(\mathrm{~d}, J=7.2 \mathrm{~Hz}, 2 \mathrm{H}), 7.71(\mathrm{t}, J=7.6 \mathrm{~Hz}, 1 \mathrm{H}), 7.56(\mathrm{t}, J=8.0 \mathrm{~Hz}, 2 \mathrm{H}), 7.41(\mathrm{~s}, 2 \mathrm{H})$, $2.66(\mathrm{~s}, 6 \mathrm{H}) .{ }^{13} \mathrm{C}\left\{{ }^{1} \mathrm{H}\right\} \mathrm{NMR}\left(100 \mathrm{MHz}, \mathrm{CDCl}_{3}\right) \delta 163.2,163.0,137.0,136.5,134.9,130.7,129.0$, 126.0, 125.7, 17.6. HRMS (ESI-TOF) $\mathrm{m} / z$ [M+Na] ${ }^{+}$calcd. for $\mathrm{C}_{18} \mathrm{H}_{13} \mathrm{NO}_{5} \mathrm{Na}^{+}$346.0691, found 346.0693.<smiles>O=C(ON1C(=O)c2c(Cl)c(Cl)c(Cl)c(Cl)c2C1=O)C(=O)c1ccccc1</smiles> 


\section{4,5,6,7-Tetrachloro-1,3-dioxoisoindolin-2-yl 2-oxo-2-phenylacetate (5c)}

Prepared according to the general procedure, $80^{\circ} \mathrm{C}, 24 \mathrm{~h}$. Purification was performed by flash column chromatography $(\mathrm{PE} / \mathrm{EA} / \mathrm{DCM}=20: 1: 1)$, white solid $(155.12 \mathrm{mg}, 72 \%) .{ }^{1} \mathrm{H} \mathrm{NMR}(400 \mathrm{MHz}$, $\left.\mathrm{CDCl}_{3}\right) \delta 8.19(\mathrm{~d}, J=7.6 \mathrm{~Hz}, 2 \mathrm{H}), 7.74(\mathrm{t}, J=7.2 \mathrm{~Hz}, 1 \mathrm{H}), 7.57(\mathrm{t}, J=8.0 \mathrm{~Hz}, 2 \mathrm{H}) .{ }^{13} \mathrm{C}\left\{{ }^{1} \mathrm{H}\right\} \mathrm{NMR}$ $\left(100 \mathrm{MHz}, \mathrm{CDCl}_{3}\right) \delta 162.5,157.8,141.2,135.3,130.8,130.6,129.1,124.9,124.9$. HRMS (ESI-TOF) $m / z[\mathrm{M}+\mathrm{Na}]^{+}$calcd. for $\mathrm{C}_{16} \mathrm{H}_{5} \mathrm{NO}_{5} \mathrm{Na}^{+} 453.8820$, found 453.8823 .<smiles>O=C(ON1C(=O)c2cccc3cccc(c23)C1=O)C(=O)c1ccccc1</smiles>

1,3-Dioxo-1 $H$-benzo[de] isoquinolin-2(3H)-yl 2-oxo-2-phenylacetate (5d)

Prepared according to the general procedure, $0.25 \mathrm{mmol}, 2.0$ equiv TBN, $80{ }^{\circ} \mathrm{C}, 24 \mathrm{~h}$. Purification was performed by flash column chromatography (PE/EA/DCM=20:1:1), white solid (37.95 mg, 44\%). ${ }^{1} \mathrm{H}$ NMR (400 MHz, $\left.\mathrm{CDCl}_{3}\right) \delta 8.67$ (dd, $\left.J=7.2,0.8 \mathrm{~Hz}, 2 \mathrm{H}\right), 8.31-8.27$ (m, $\left.4 \mathrm{H}\right), 7.83$ (t, $J=7.6 \mathrm{~Hz}$, $2 \mathrm{H}), 7.72(\mathrm{t}, J=7.6 \mathrm{~Hz}, 1 \mathrm{H}), 7.57(\mathrm{t}, J=8.0 \mathrm{~Hz}, 2 \mathrm{H}) .{ }^{13} \mathrm{C}\left\{{ }^{1} \mathrm{H}\right\} \mathrm{NMR}\left(100 \mathrm{MHz}, \mathrm{CDCl}_{3}\right) \delta 163.1$, $159.9,135.2$, 134.7, 132.2, 132.1, 130.8, 128.9, 127.9, 127.3, 126.3, 122.6. HRMS (ESI-TOF) $\mathrm{m} / \mathrm{z}$ $[\mathrm{M}+\mathrm{Na}]^{+}$calcd. for $\mathrm{C}_{20} \mathrm{H}_{11} \mathrm{NO}_{5} \mathrm{Na}^{+} 368.0535$, found 368.0538 .

\subsection{General Procedure for the $30 \mathrm{mmol}$ Scale Reaction of $1 \mathrm{i}$ and $2 \mathrm{a}$}<smiles>O=CC1CCCCC1</smiles>

1i, $45 \mathrm{mmol}$

2a, $30 \mathrm{mmol}$<smiles>O=C(ON1C(=O)c2ccccc2C1=O)C1CCCCC1</smiles>

$3 \mathrm{i}, 7.79 \mathrm{~g}, 95 \%$

$N$-hydroxyphthalimide (NHPI) (4.9 g, $30.0 \mathrm{mmol}, 1.0$ equiv) was weighed directly into a $200 \mathrm{~mL}$ Schlenk tube equipped with a magnetic stir bar. After dried in vacuo for 15 min and charged with nitrogen gas, dry MeCN (100 mL) was added. Then cyclohexanecarboxaldehyde (5.6 mL, $45.0 \mathrm{mmol}$, 1.5 equiv) was added dropwise into the mixture. ${ }^{t} \mathrm{BuONO}$ (TBN) $(3.8 \mathrm{~mL}, 30.0 \mathrm{mmol}, 1.0$ equiv) was last added and the tube was capped with a glass cap. The resulting solution was stirred at $25^{\circ} \mathrm{C}$ for 12 h. Then, the solvent was removed under vacuum and the residue was purified by flash column chromatography on silica gel (PE/EA/DCM $=20: 1: 1)$ to give the 1,3-dioxoisoindolin-2-yl cyclohexanecarboxylate 3i (7.79 g, 95\%). 


\section{References}

(1). Xu, X.; Sun, J.; Lin, Y.; Cheng, J.; Li, P.; Jiang, X.; Bai, R.; Xie, Y. Eur. J. Org. Chem. 2017, 2017, $7160-7166$.

(2). Mills, L. R.; Zhou, C.; Fung, E.; Rousseaux, S. A. L. Org. Lett. 2019, 21, 8805-8809.

(3). Chan, C. M.; Xing, Q.;Chow, Y. C.; Hung, S. F.; Yu, W. Y. Org. Lett. 2019, 21, 8037-8043.

(4). Barzano, G.; Mao, R.; Garreau, M.; Waser, J.; Hu, X. Org. Lett. 2020, 22, 5412-5416.

(5). Ni, S.; Padial, N. M.; Kingston, C.; Vantourout, J. C.; Schmitt, D. C.; Edwards, J. T.; Kruszyk, M. M.; Merchant, R. R.; Mykhailiuk, P. K.; Sanchez, B. B.; Yang, S.; Perry, M. A.; Gallego, G. M.; Mousseau, J. J.; Collins, M. R.; Cherney, R. J.; Lebed, P. S.; Chen, J. S.; Qin, T.; Baran, P. S. J. Am.Chem. Soc. 2019, 141, 6726-6739.

(6). Cheng, W.-M.; Shang, R.; Zhao, B.; Xing, W.-L.; Fu, Y. Org. Lett. 2017, 19, 4291-4294.

(7). Xue, W.; Oestreich, M. Angew. Chem., Int. Ed. 2017, 56, 11649-11652.

(8). Qin, T.; Cornella, J.; Li, C.; Malins, L. R.; Edwards, J. T.; Kawamura, S.; Maxwell, B. D.; Eastgate, M. D.; Baran, P. S. Science 2016, 352, 801-805. 


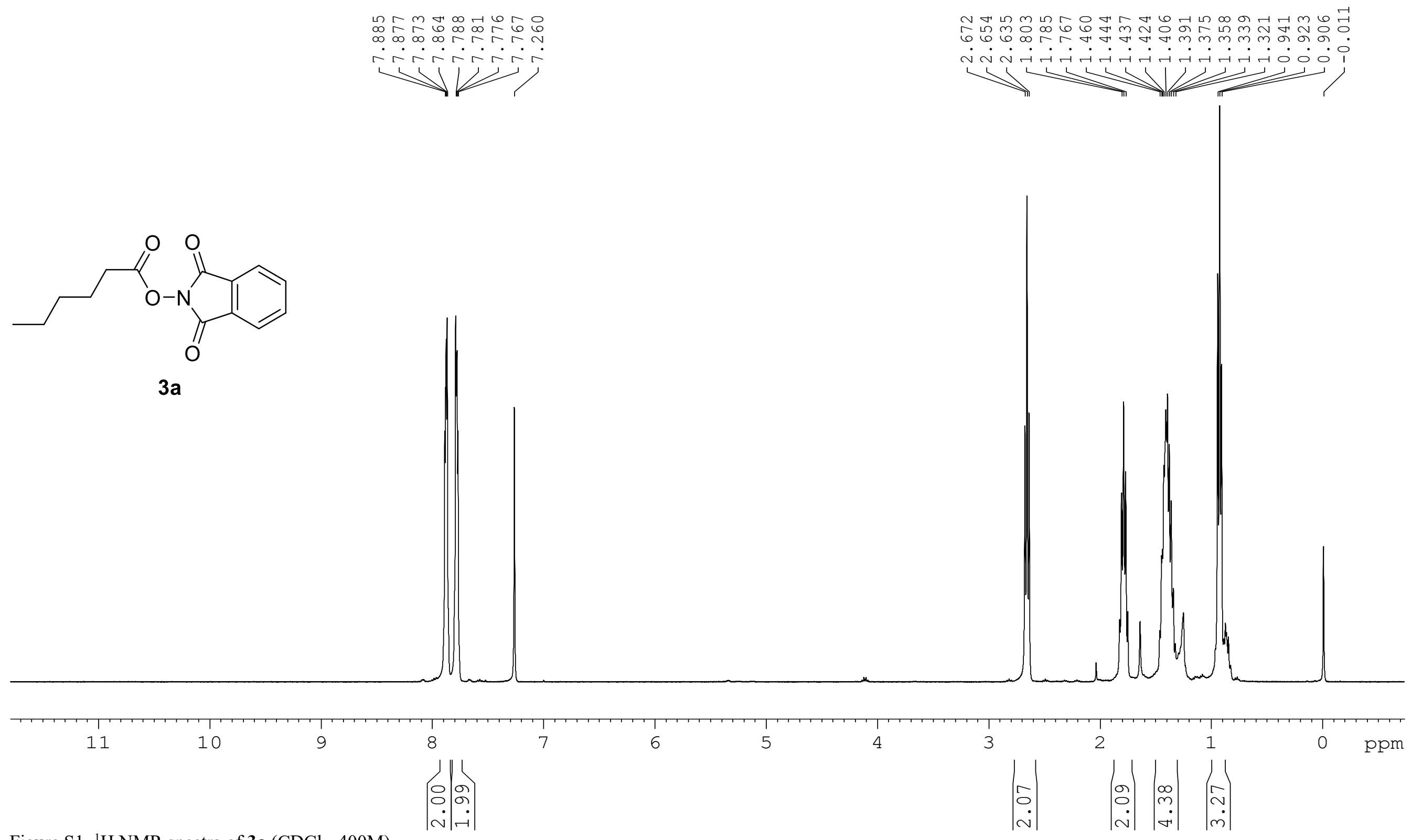

Figure S1. ${ }^{1} \mathrm{H}$ NMR spectra of 3a $\left(\mathrm{CDCl}_{3}, 400 \mathrm{M}\right)$. 

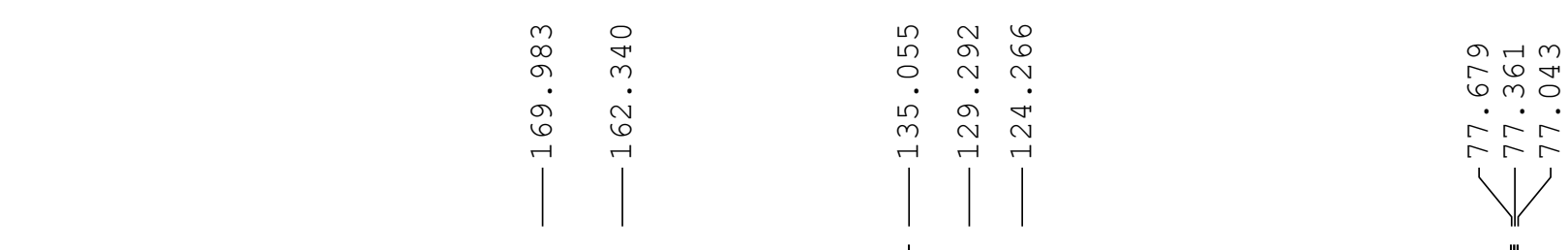

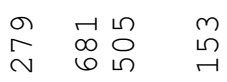

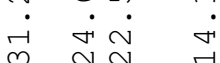

111

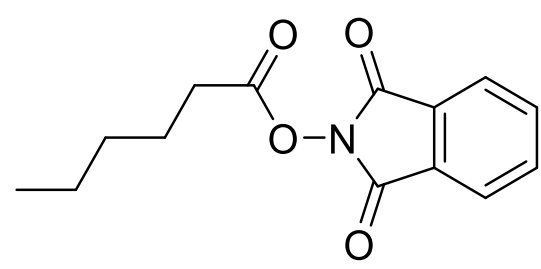

$3 a$
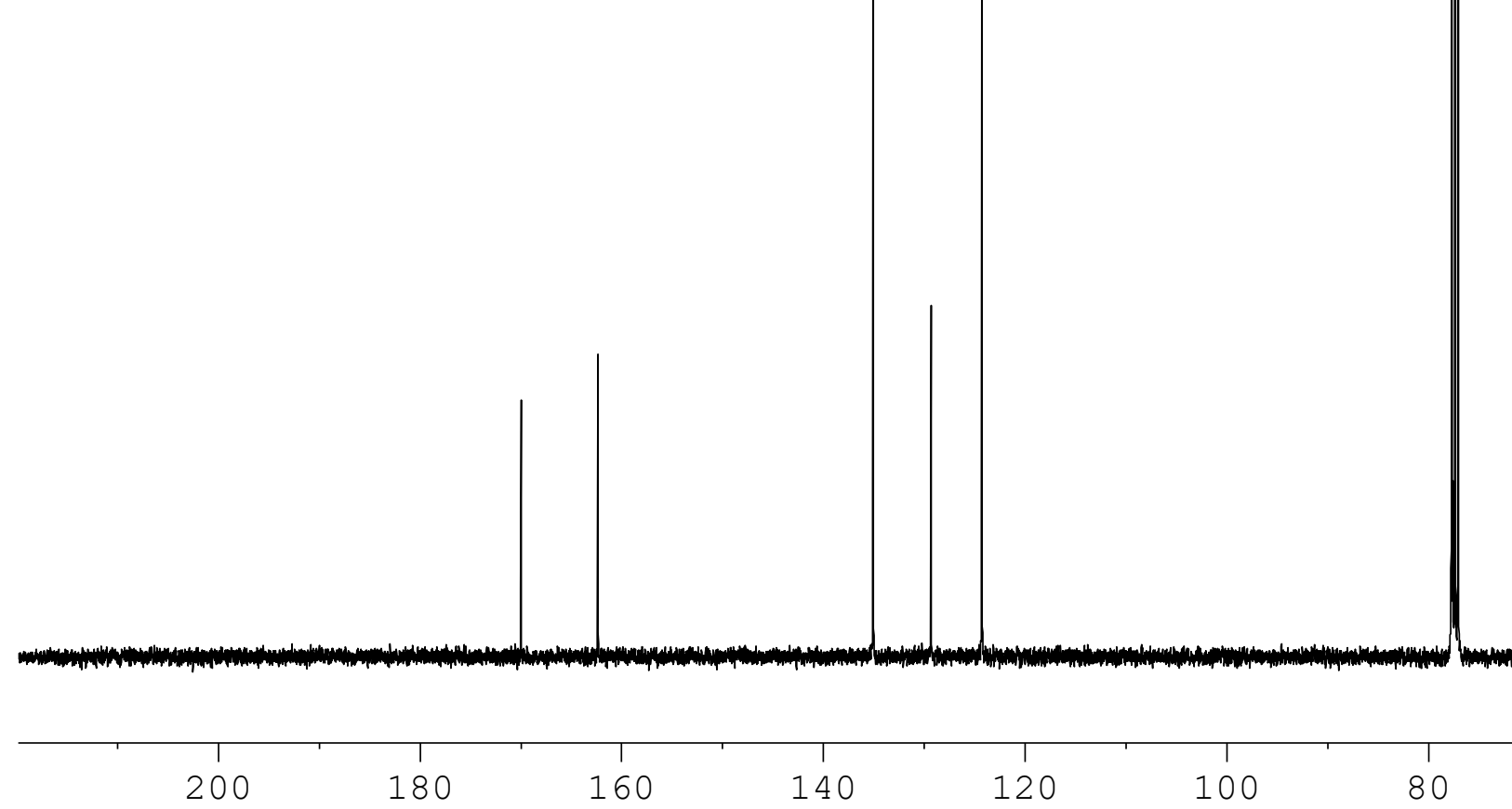

140

120

100

80

60

40

20

ppm

Figure S2. ${ }^{13} \mathrm{C}$ NMR spectra of $\mathbf{3 a}\left(\mathrm{CDCl}_{3}, 100 \mathrm{M}\right)$. 


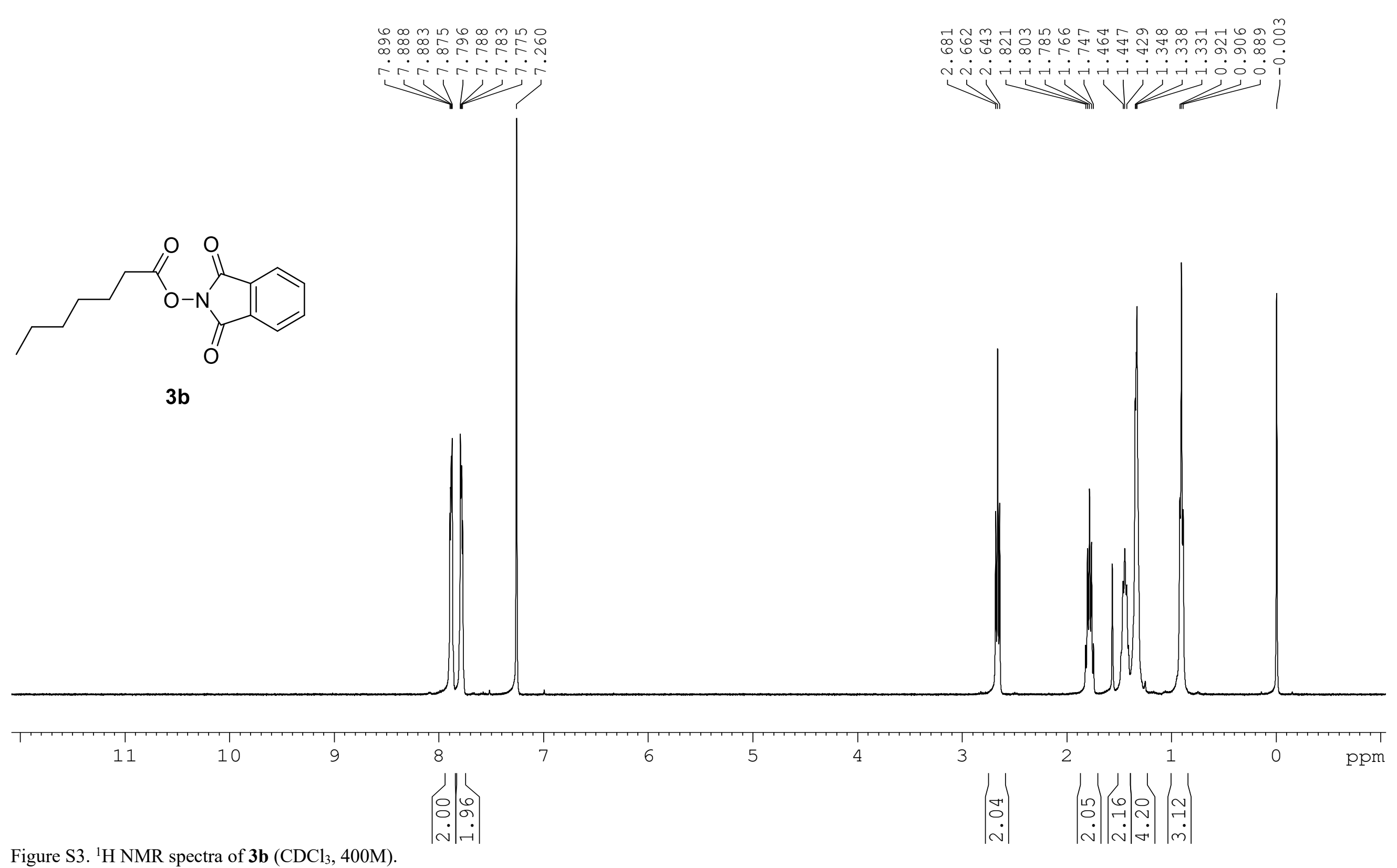



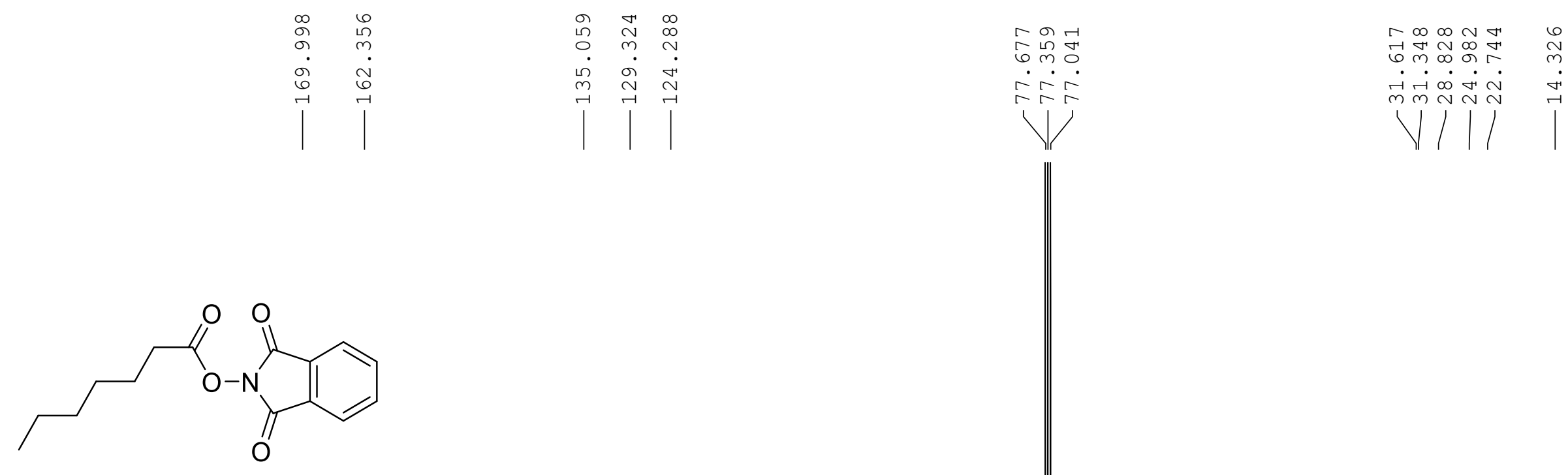

$3 b$

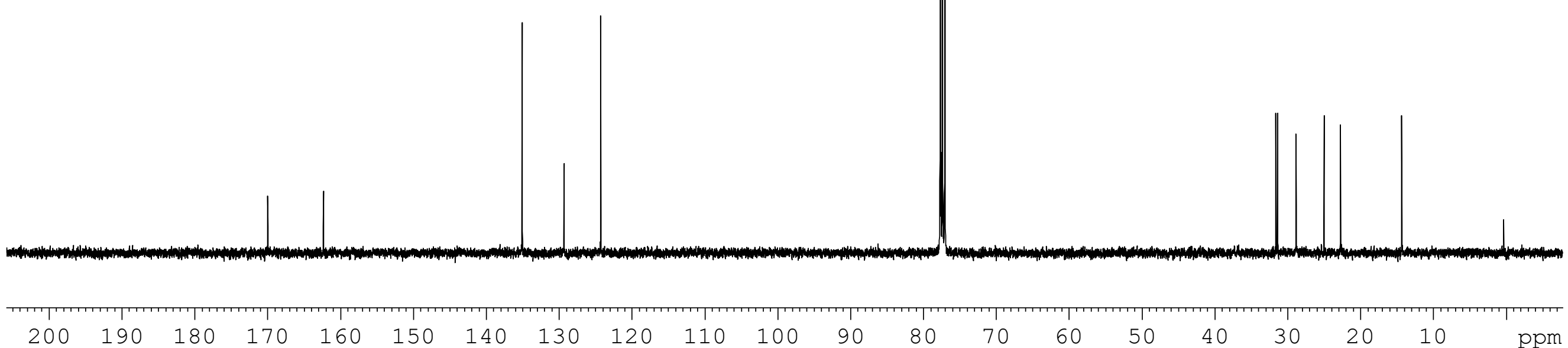
Figure $\mathrm{S} 4 .{ }^{13} \mathrm{C}$ NMR spectra of $\mathbf{3 b}\left(\mathrm{CDCl}_{3}, 100 \mathrm{M}\right)$. 


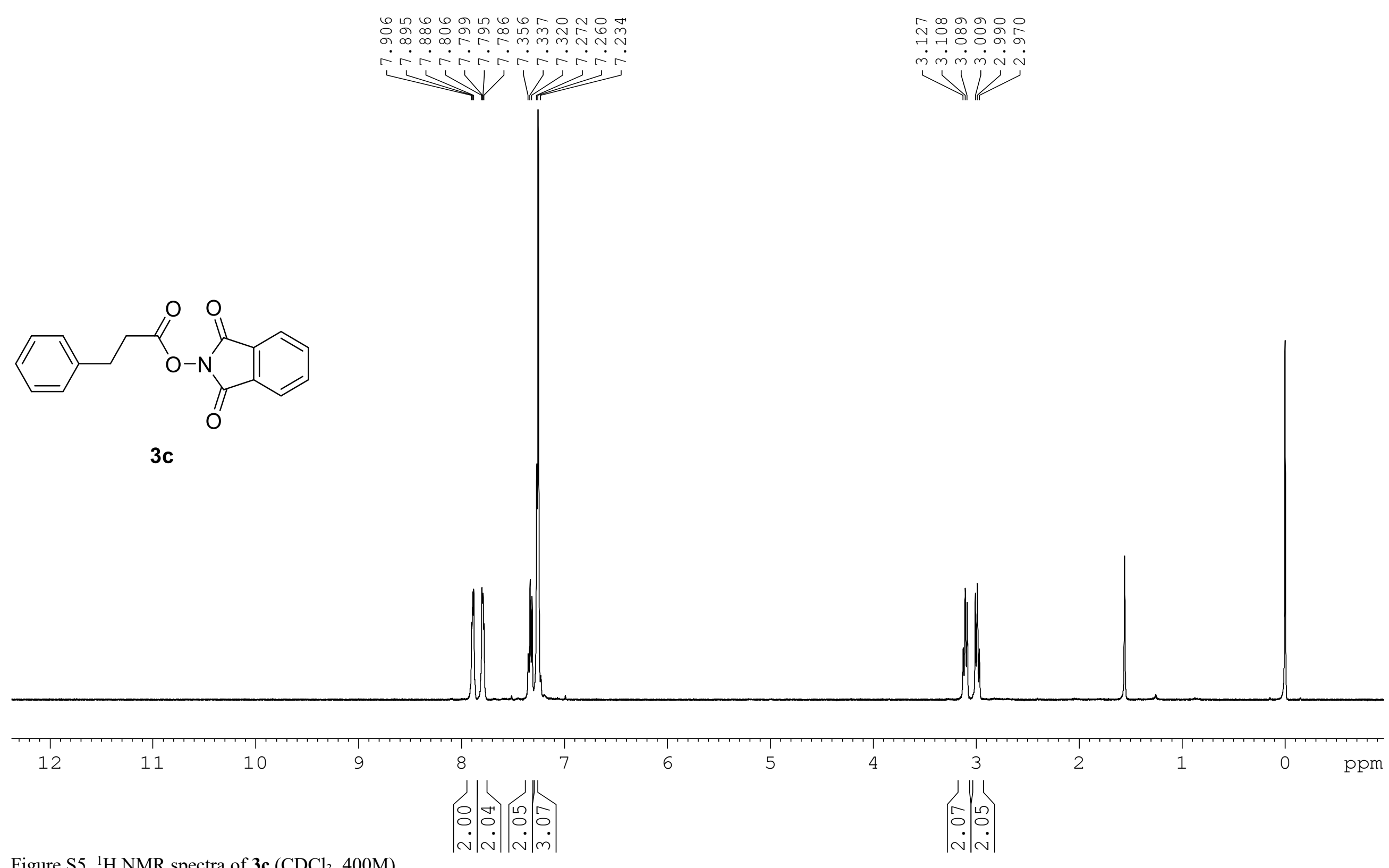

Figure S5. ${ }^{1} \mathrm{H}$ NMR spectra of $3 \mathbf{c}\left(\mathrm{CDCl}_{3}, 400 \mathrm{M}\right)$. 


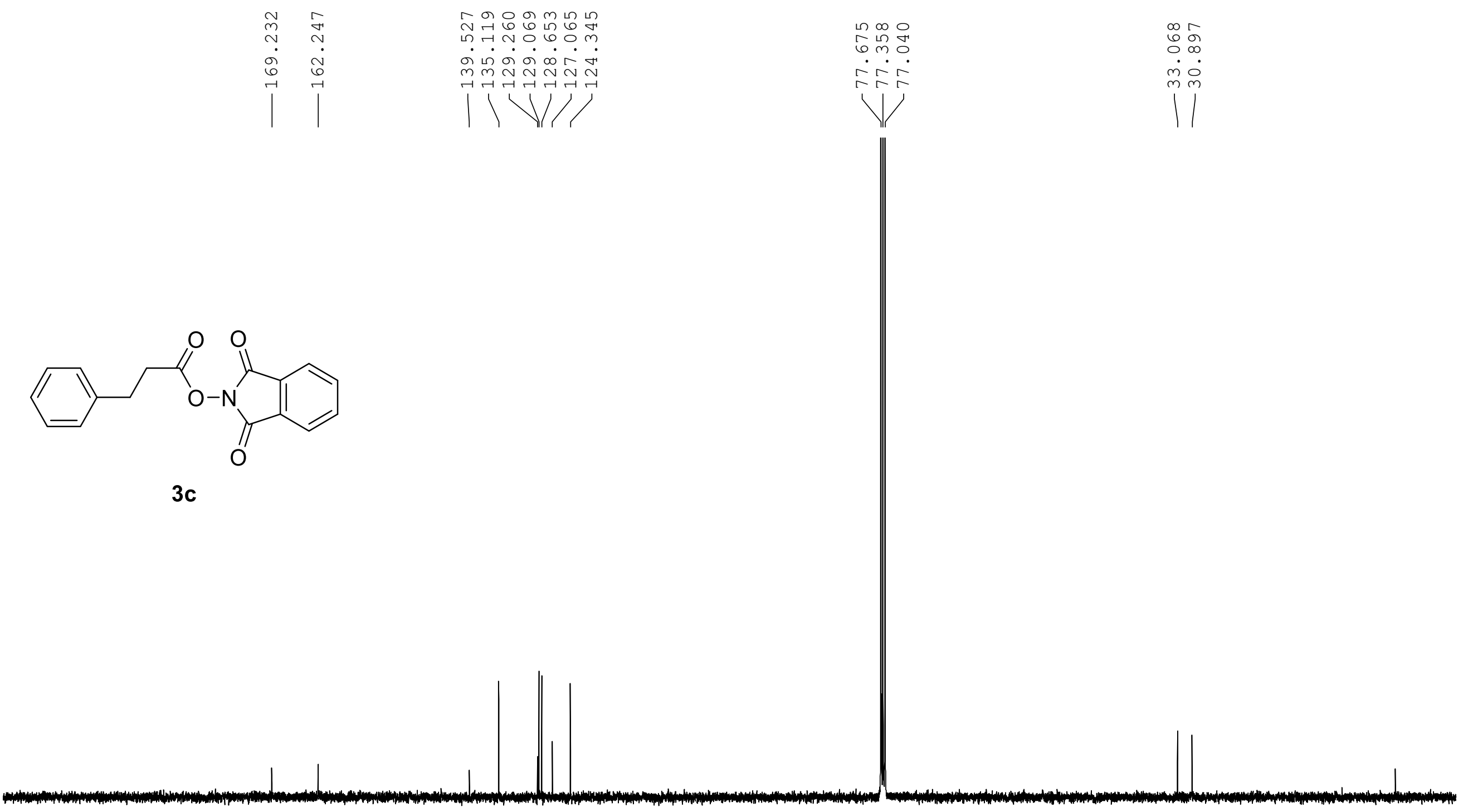

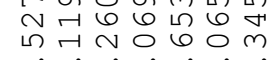

向

$\because$ :

$m o$

1

$3 c$

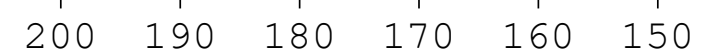

Figure S6. ${ }^{13} \mathrm{C}$ NMR spectra of $\mathbf{3 c}\left(\mathrm{CDCl}_{3}, 100 \mathrm{M}\right)$. 


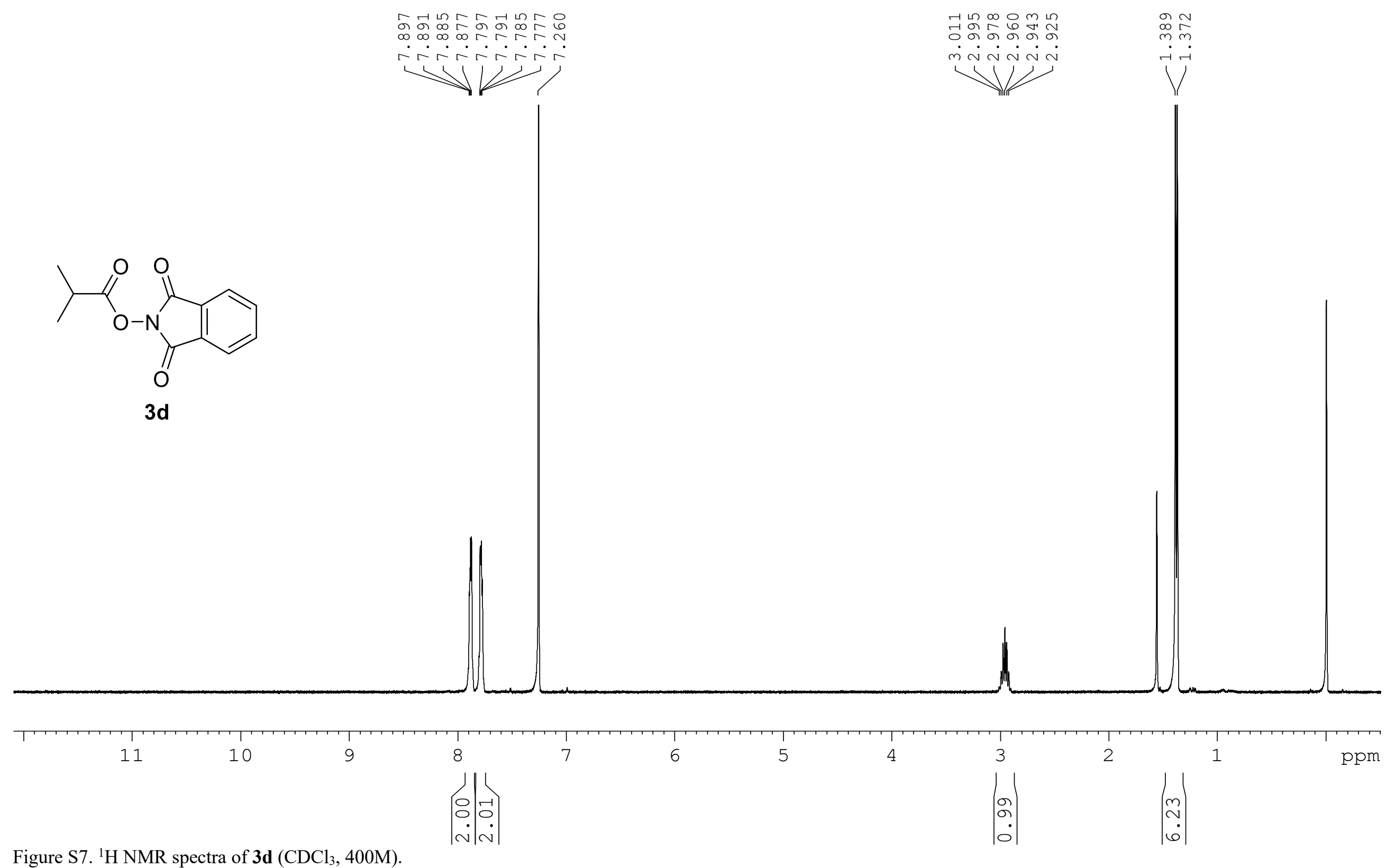




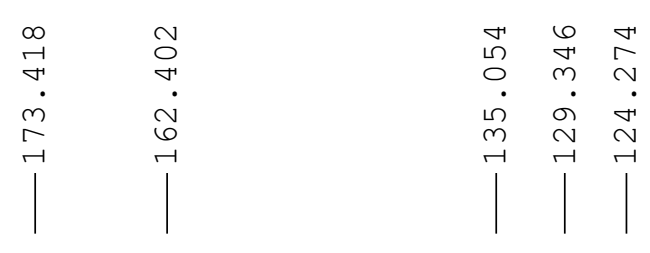
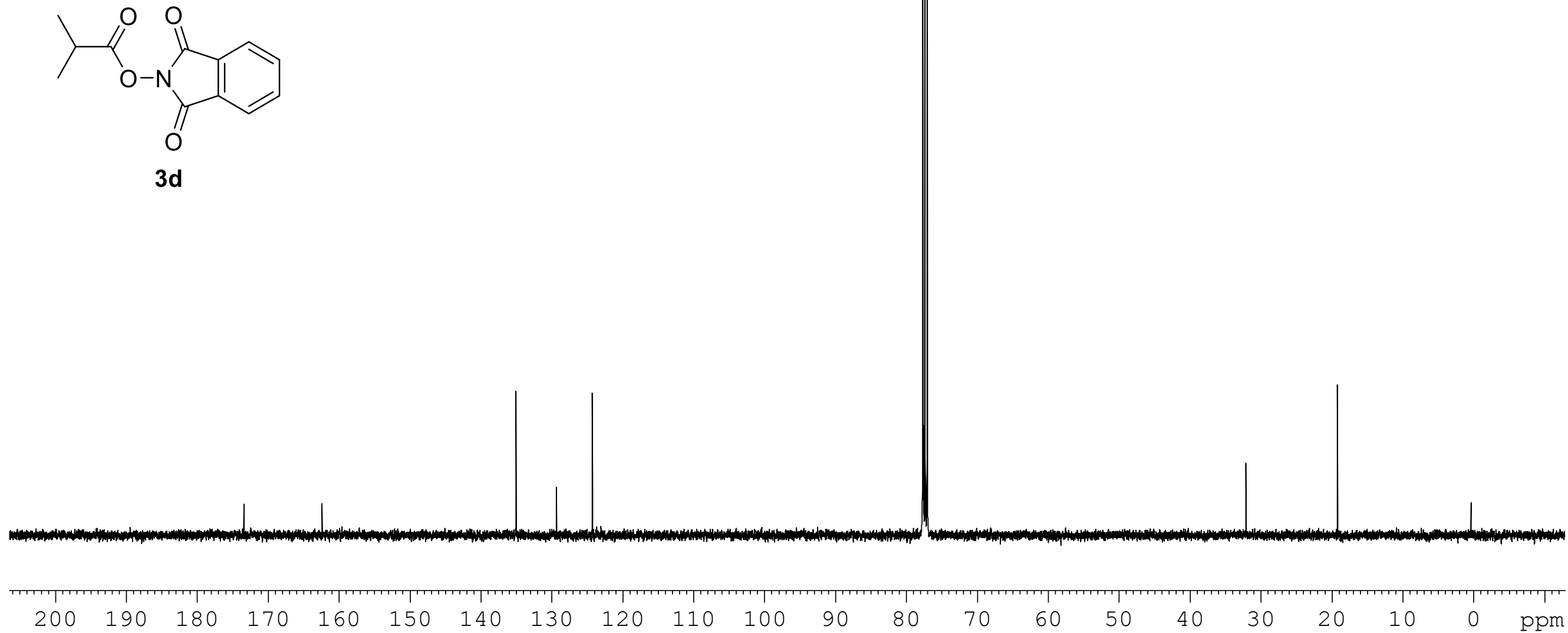

Figure $\mathrm{S} 8 .{ }^{13} \mathrm{C}$ NMR spectra of $\mathbf{3 d}\left(\mathrm{CDCl}_{3}, 100 \mathrm{M}\right)$. 

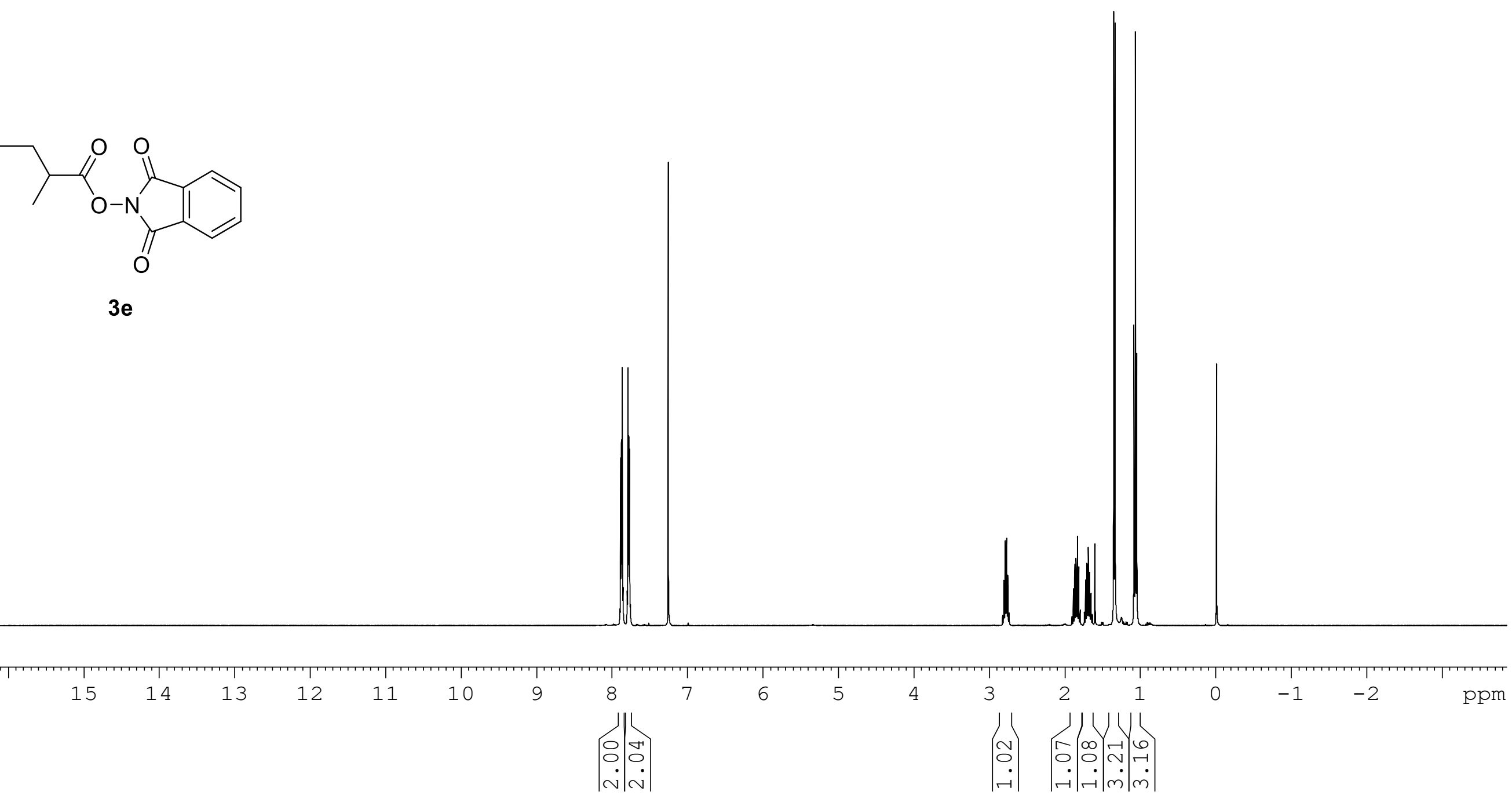

Figure S9. ${ }^{1} \mathrm{H}$ NMR spectra of $\mathbf{3 e}\left(\mathrm{CDCl}_{3}, 400 \mathrm{M}\right)$.

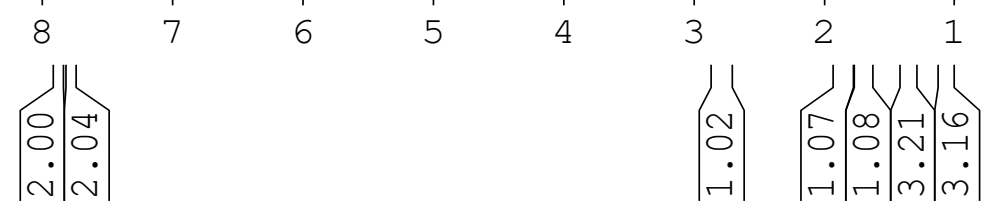



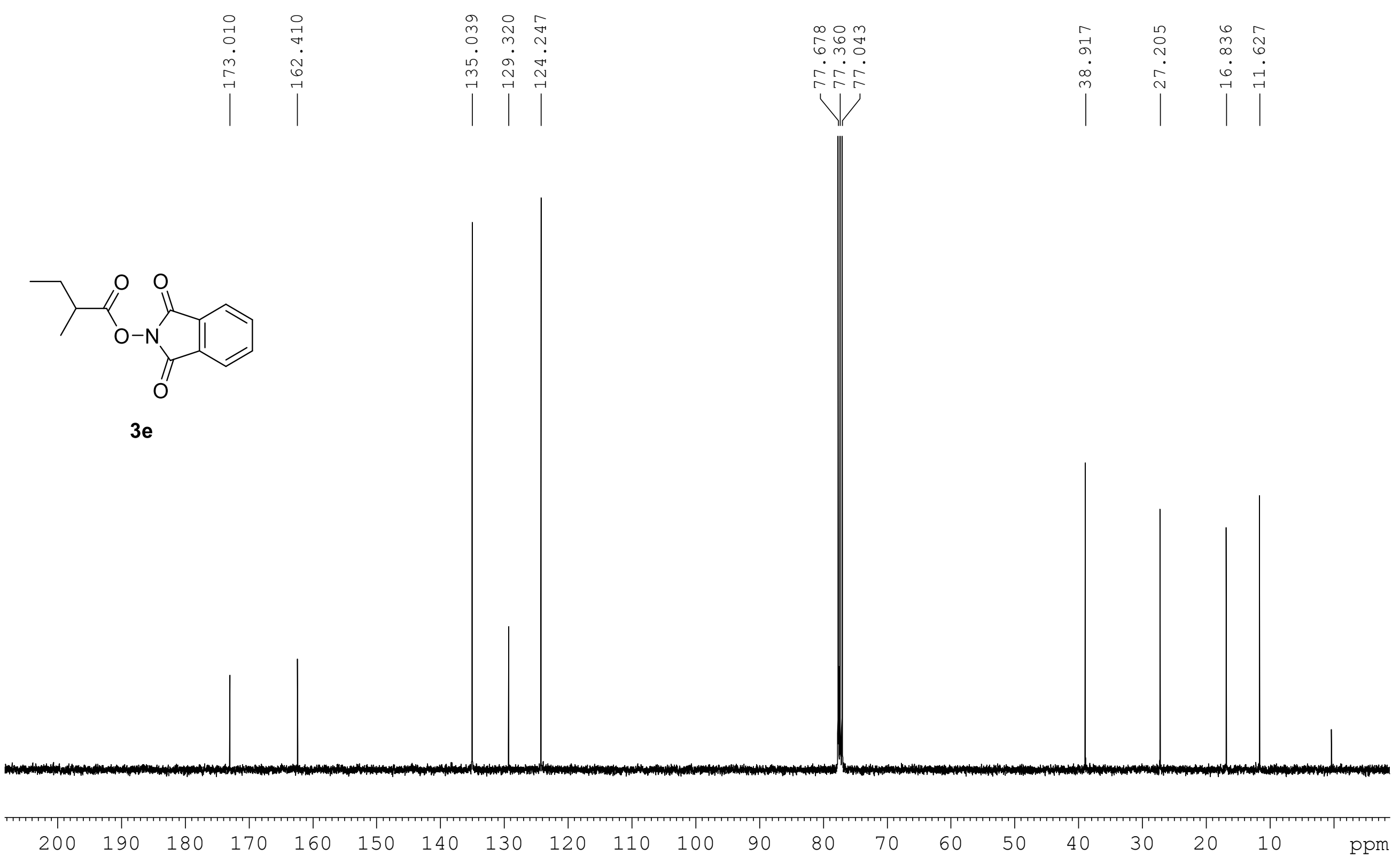
Figure $\mathrm{S} 10 .{ }^{13} \mathrm{C} \mathrm{NMR}$ spectra of $\mathbf{3 e}\left(\mathrm{CDCl}_{3}, 100 \mathrm{M}\right)$. 


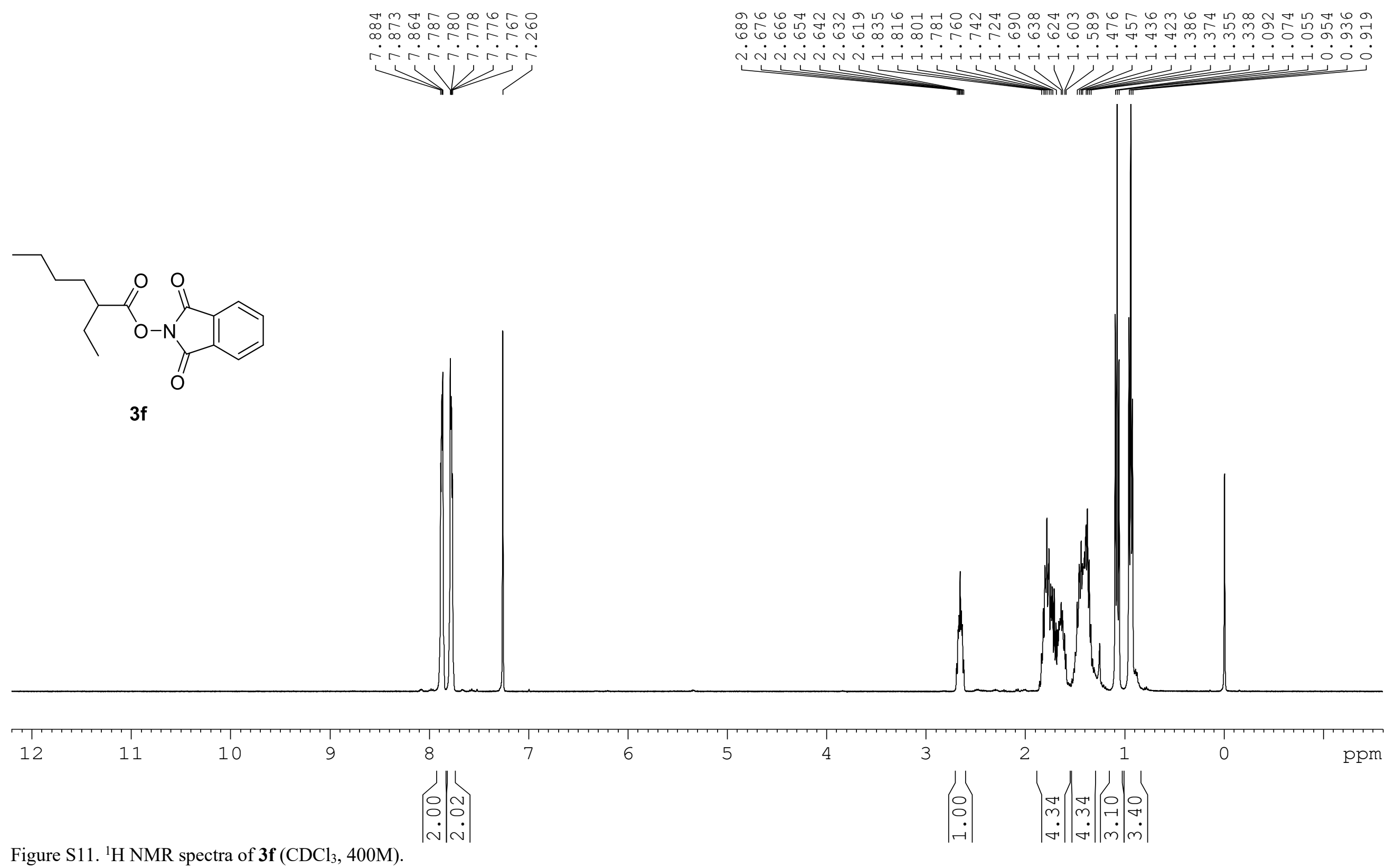



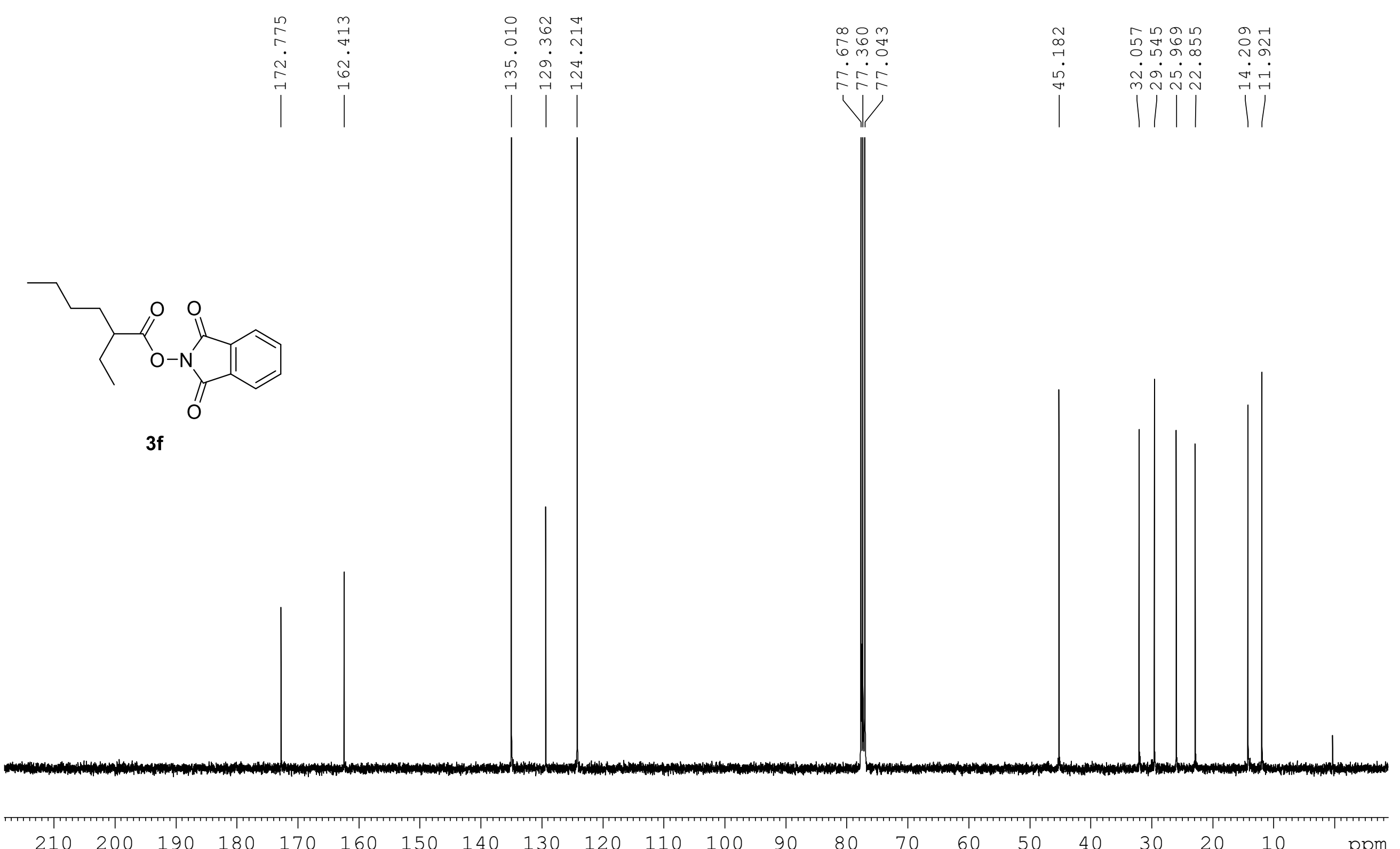

Figure $\mathrm{S} 12 .{ }^{13} \mathrm{C}$ NMR spectra of $\mathbf{3 f}\left(\mathrm{CDCl}_{3}, 100 \mathrm{M}\right)$. 


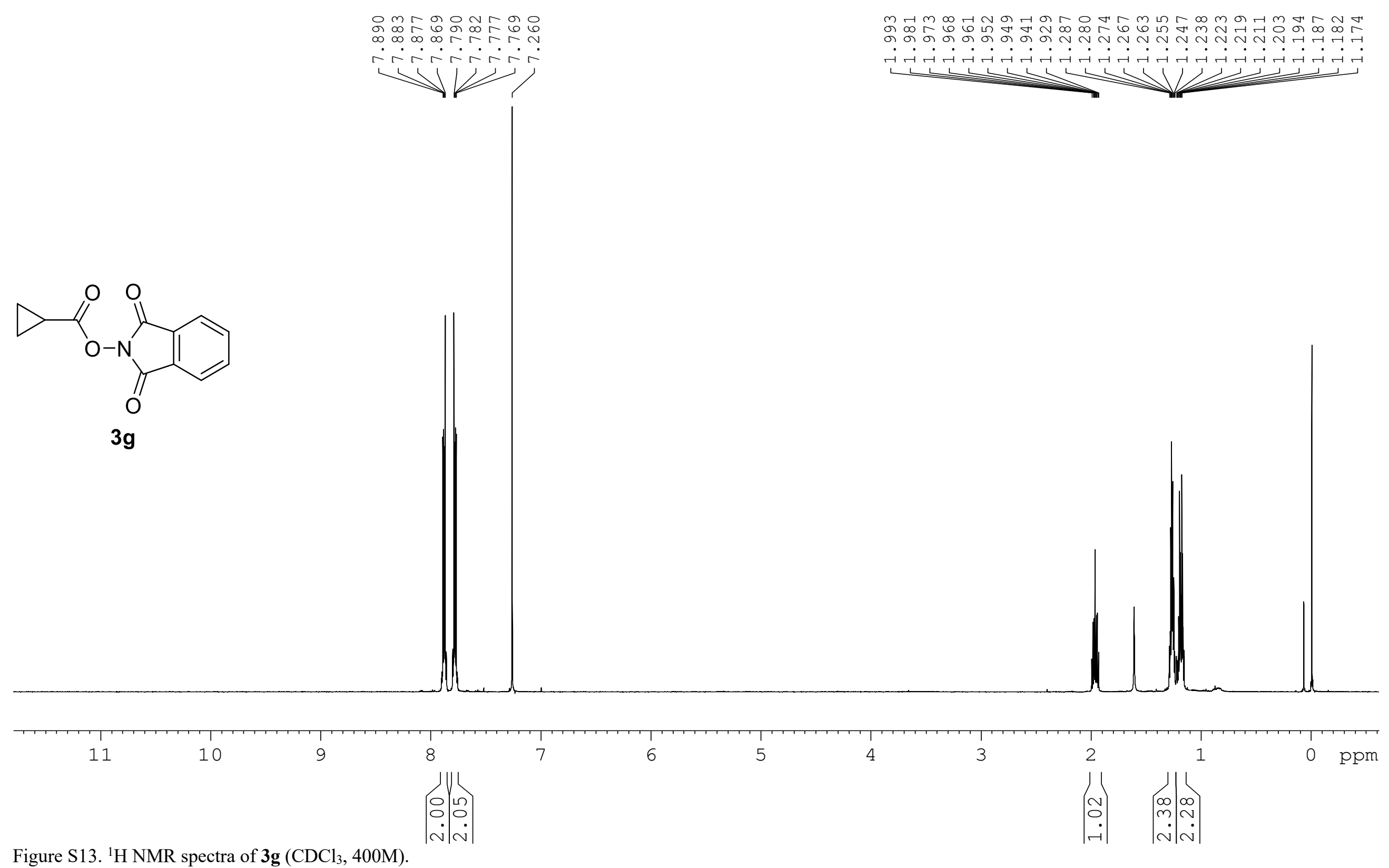



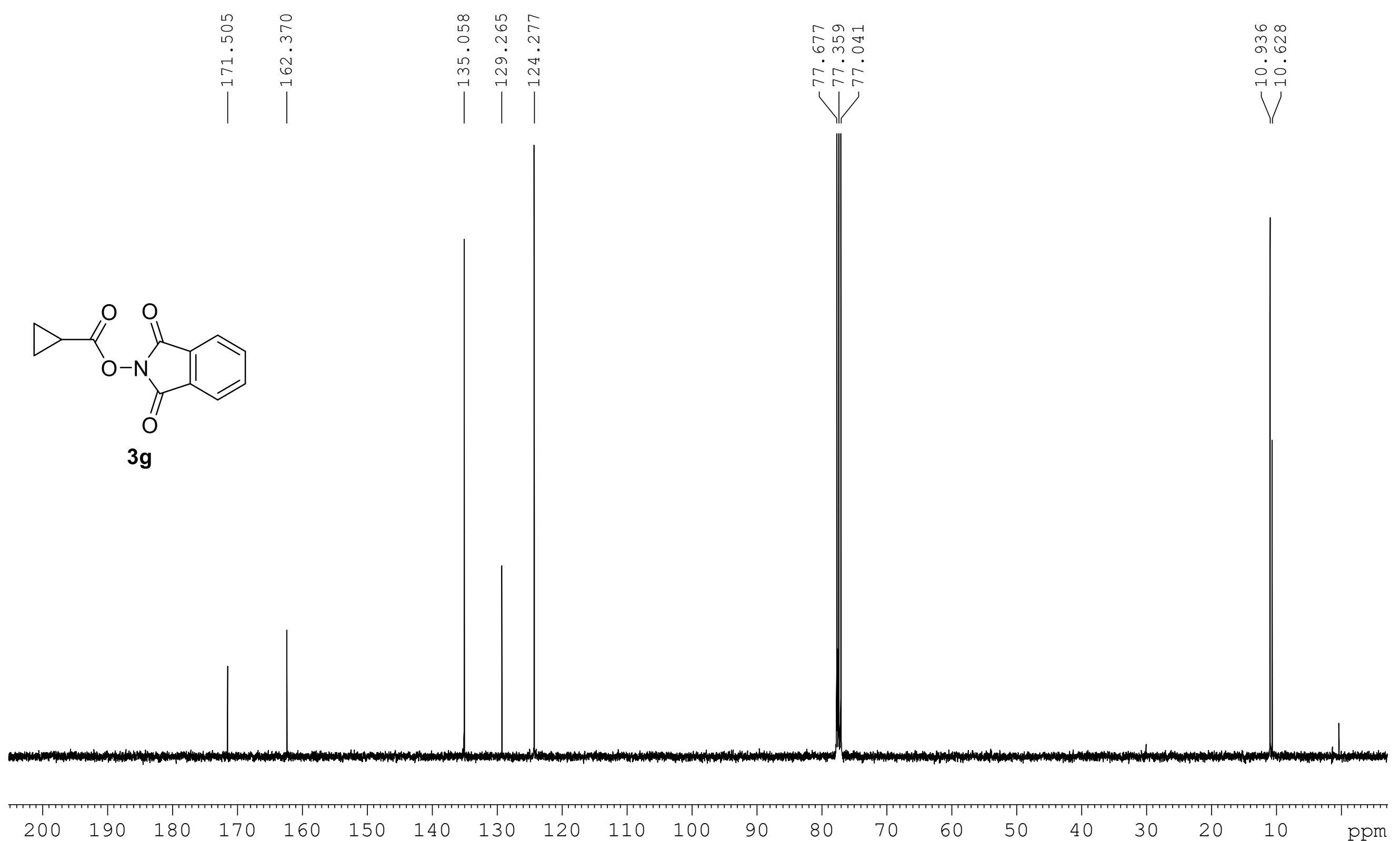

Figure S14. ${ }^{13} \mathrm{C}$ NMR spectra of $\mathbf{3 g}\left(\mathrm{CDCl}_{3}, 100 \mathrm{M}\right)$. 
<smiles>O=C(ON1C(=O)c2ccccc2C1=O)C1CCCC1</smiles>

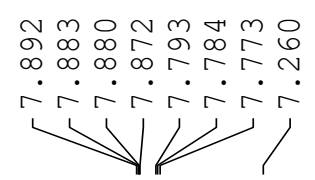

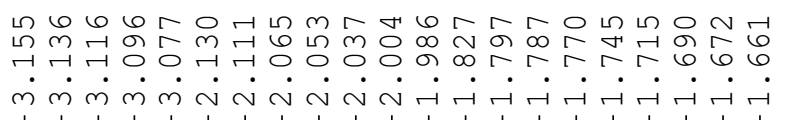

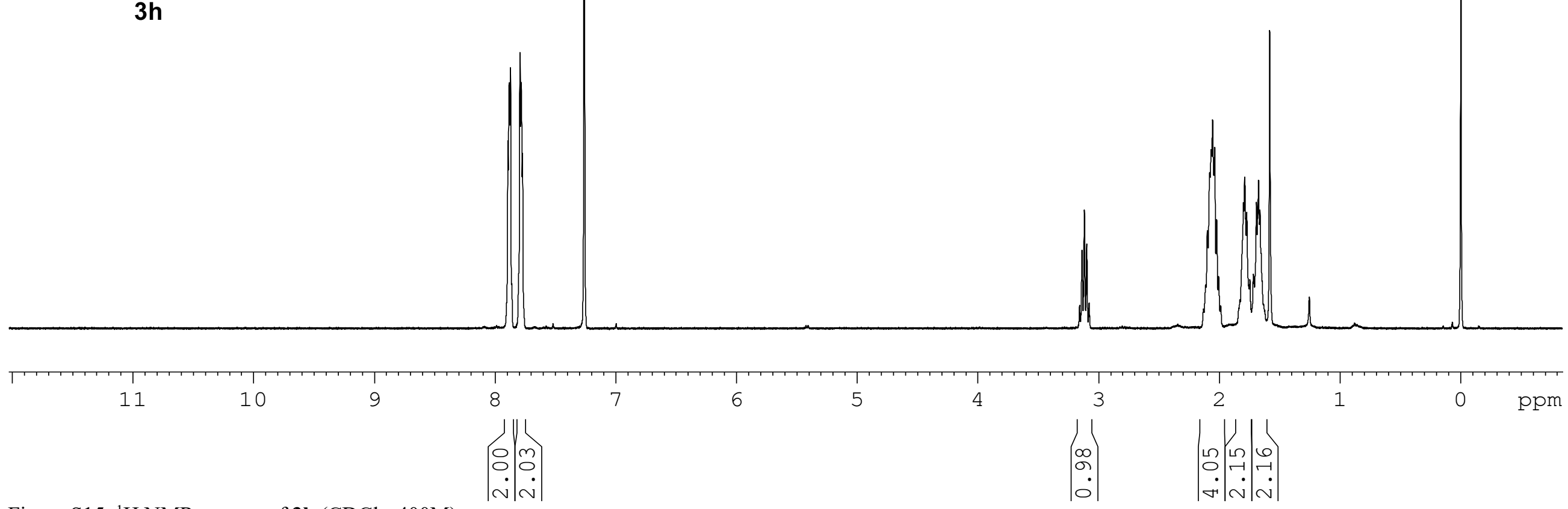

Figure S15. ${ }^{1} \mathrm{H}$ NMR spectra of $\mathbf{3 h}\left(\mathrm{CDCl}_{3}, 400 \mathrm{M}\right)$.

S-33 

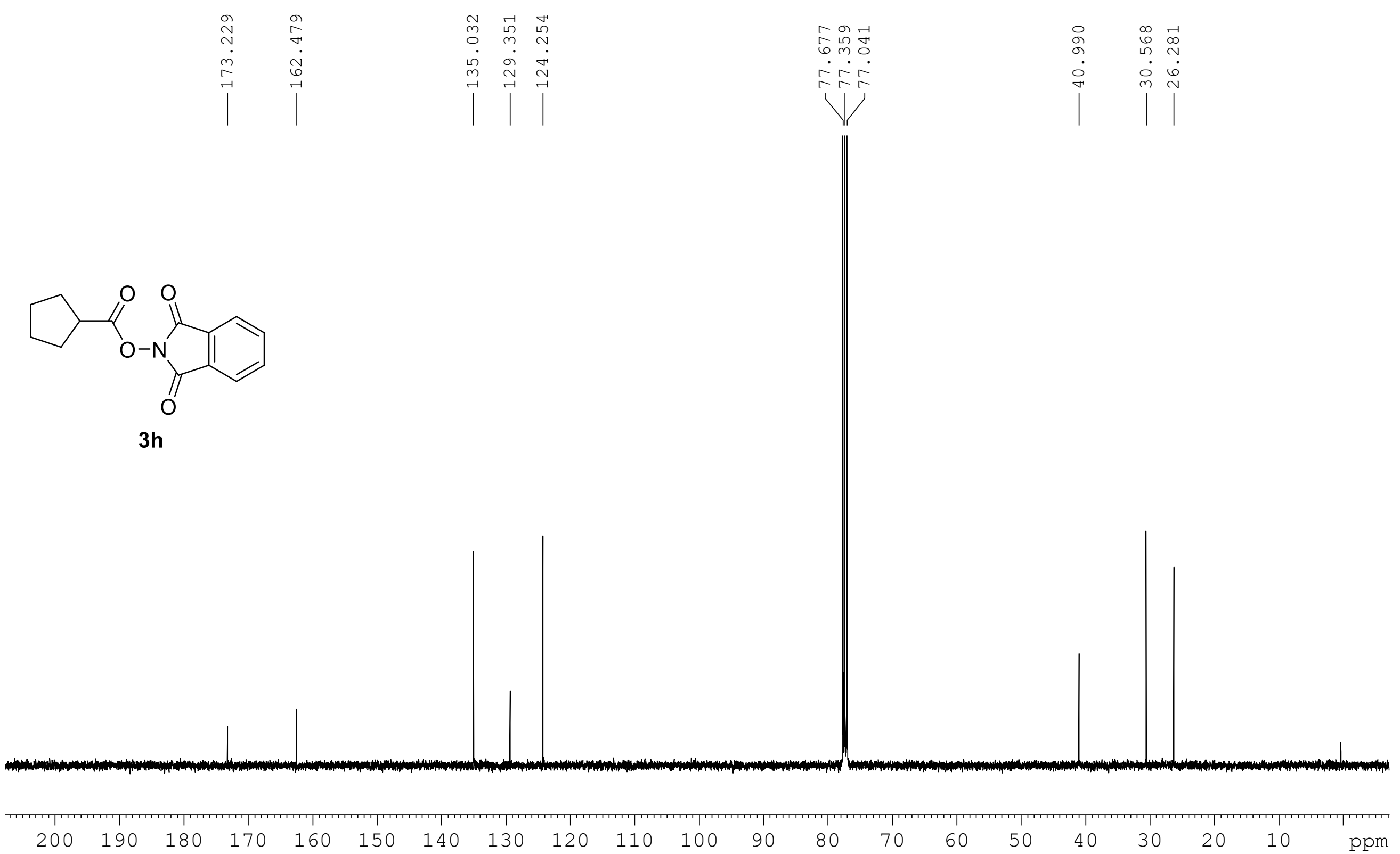

Figure S16. ${ }^{13} \mathrm{C}$ NMR spectra of $\mathbf{3 h}\left(\mathrm{CDCl}_{3}, 100 \mathrm{M}\right)$. 


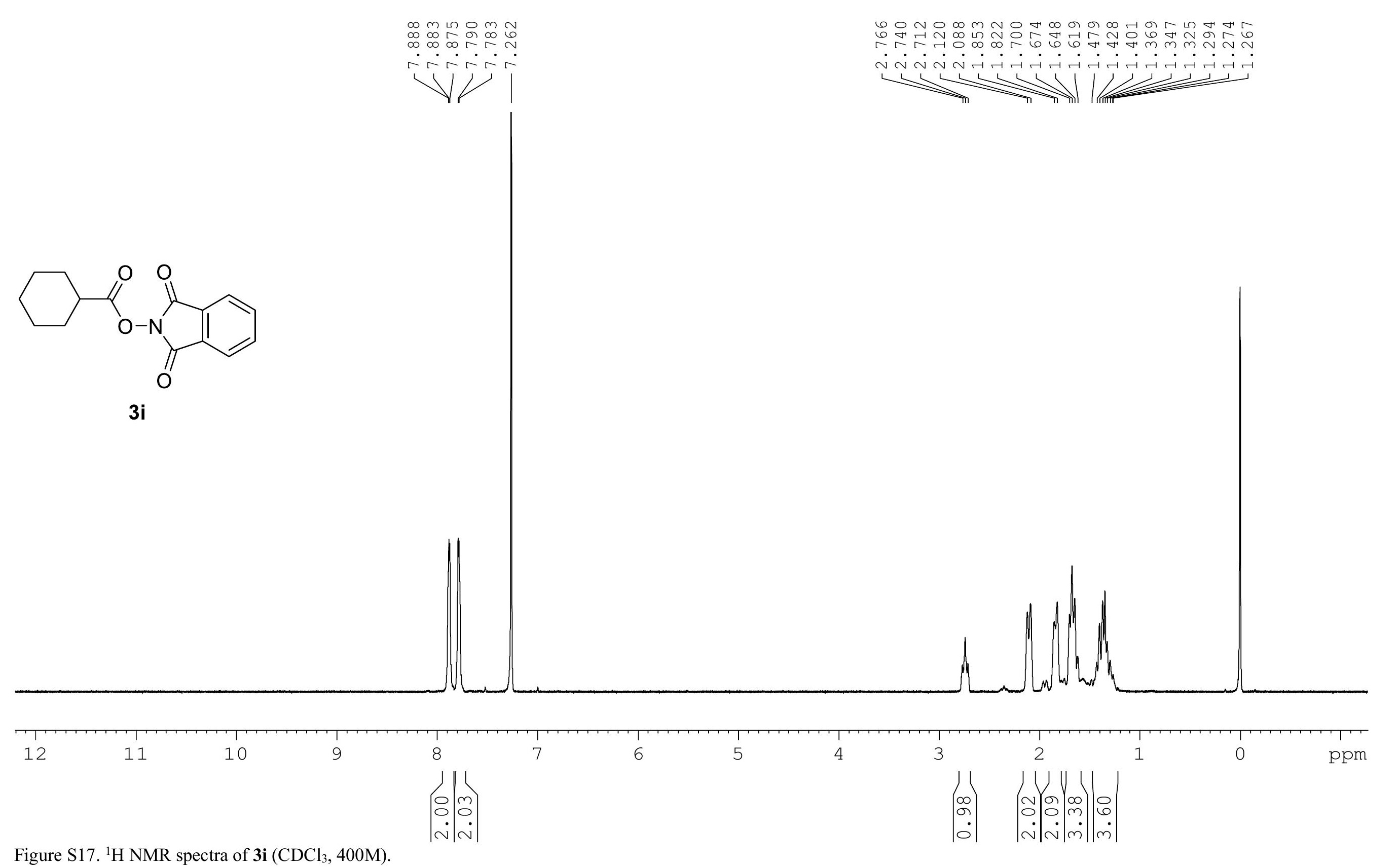




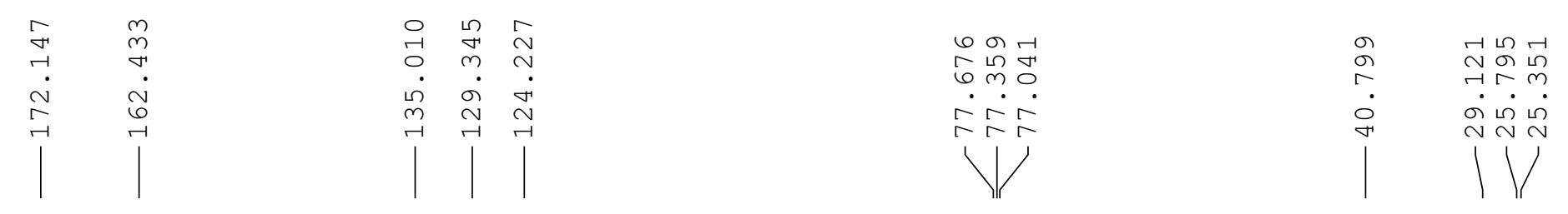

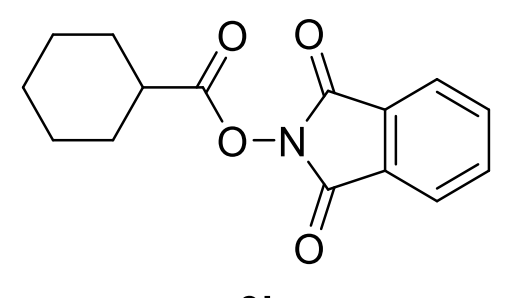

3i

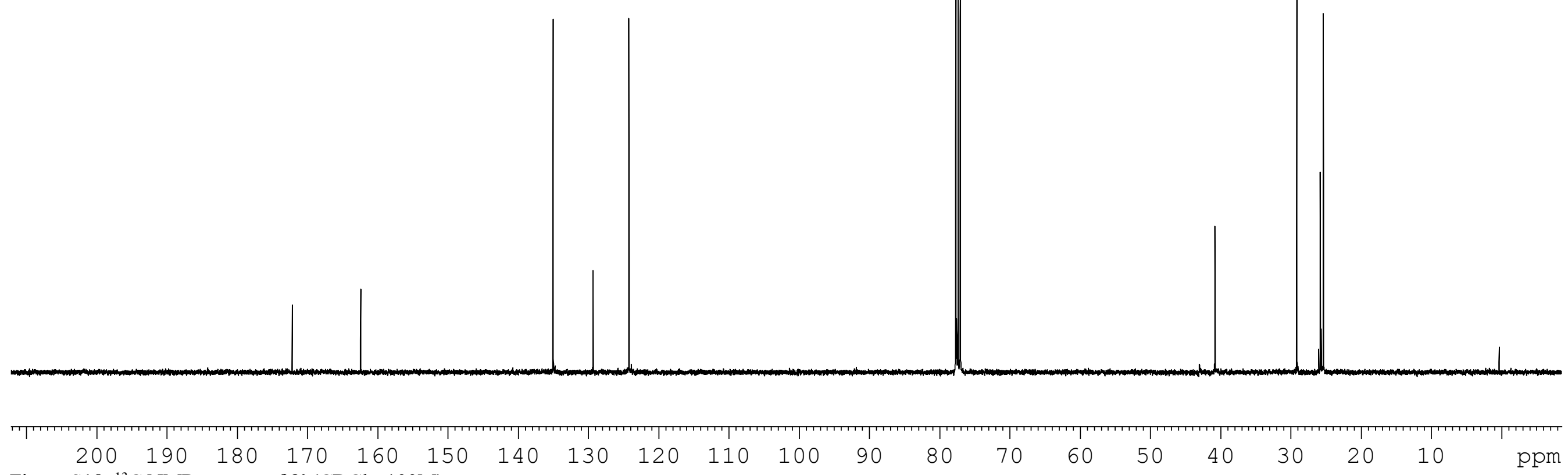

Figure $\mathrm{S} 18 .{ }^{13} \mathrm{C}$ NMR spectra of $\mathbf{3 i}\left(\mathrm{CDCl}_{3}, 100 \mathrm{M}\right)$. 


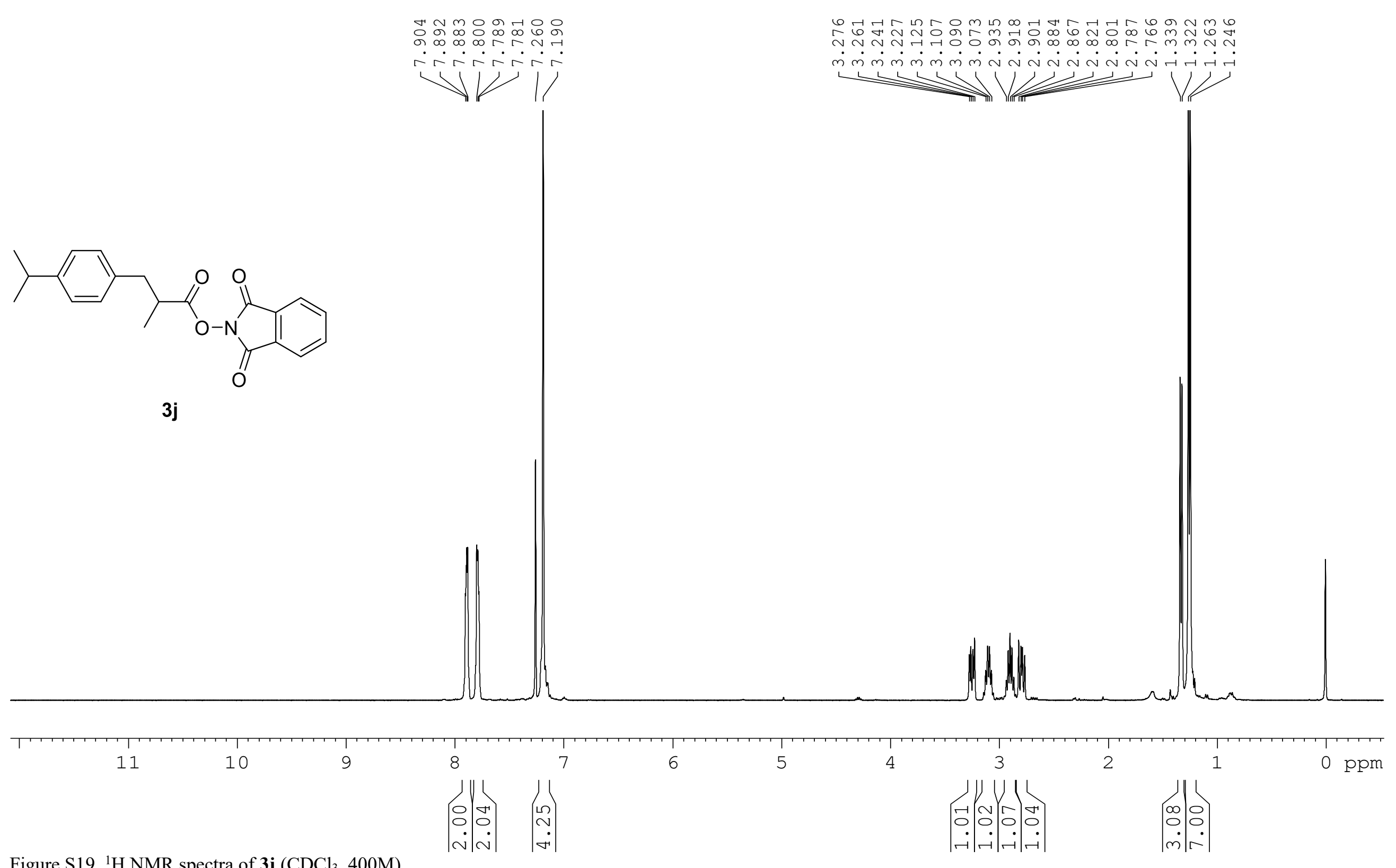

Figure S19. ${ }^{1} \mathrm{H}$ NMR spectra of $\mathbf{3 j}\left(\mathrm{CDCl}_{3}, 400 \mathrm{M}\right)$. 


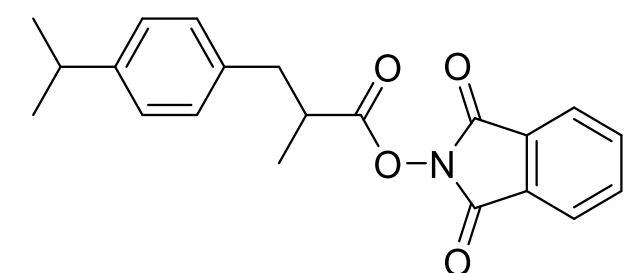

3j

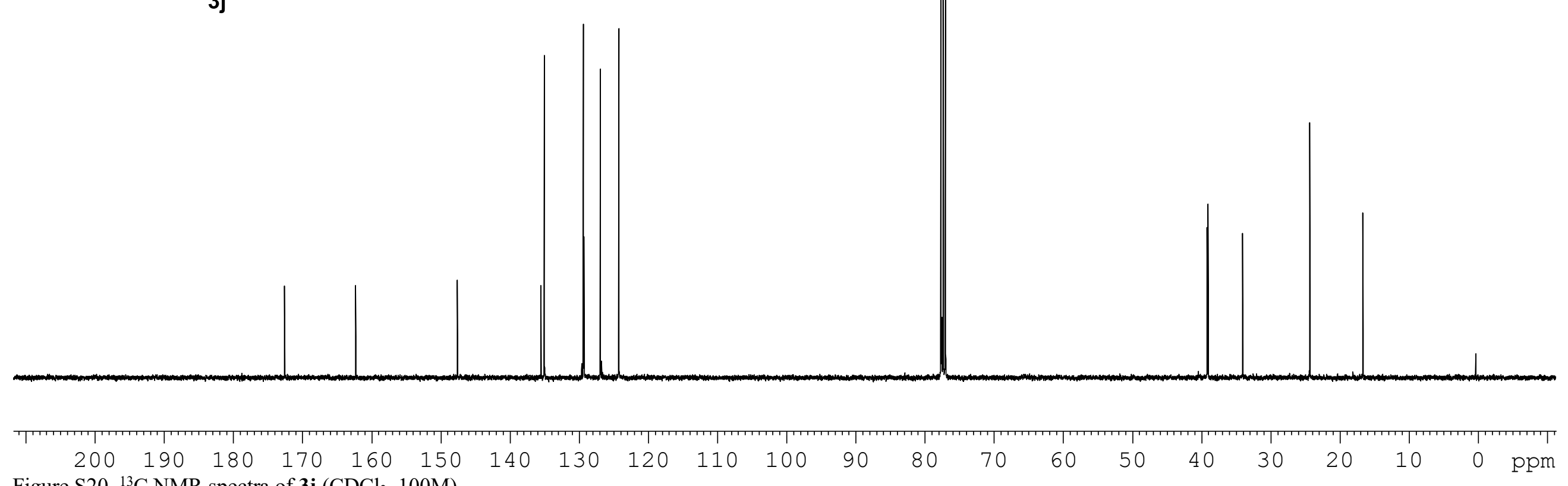

Figure S20. ${ }^{13} \mathrm{C}$ NMR spectra of $\mathbf{3 j}\left(\mathrm{CDCl}_{3}, 100 \mathrm{M}\right)$. 


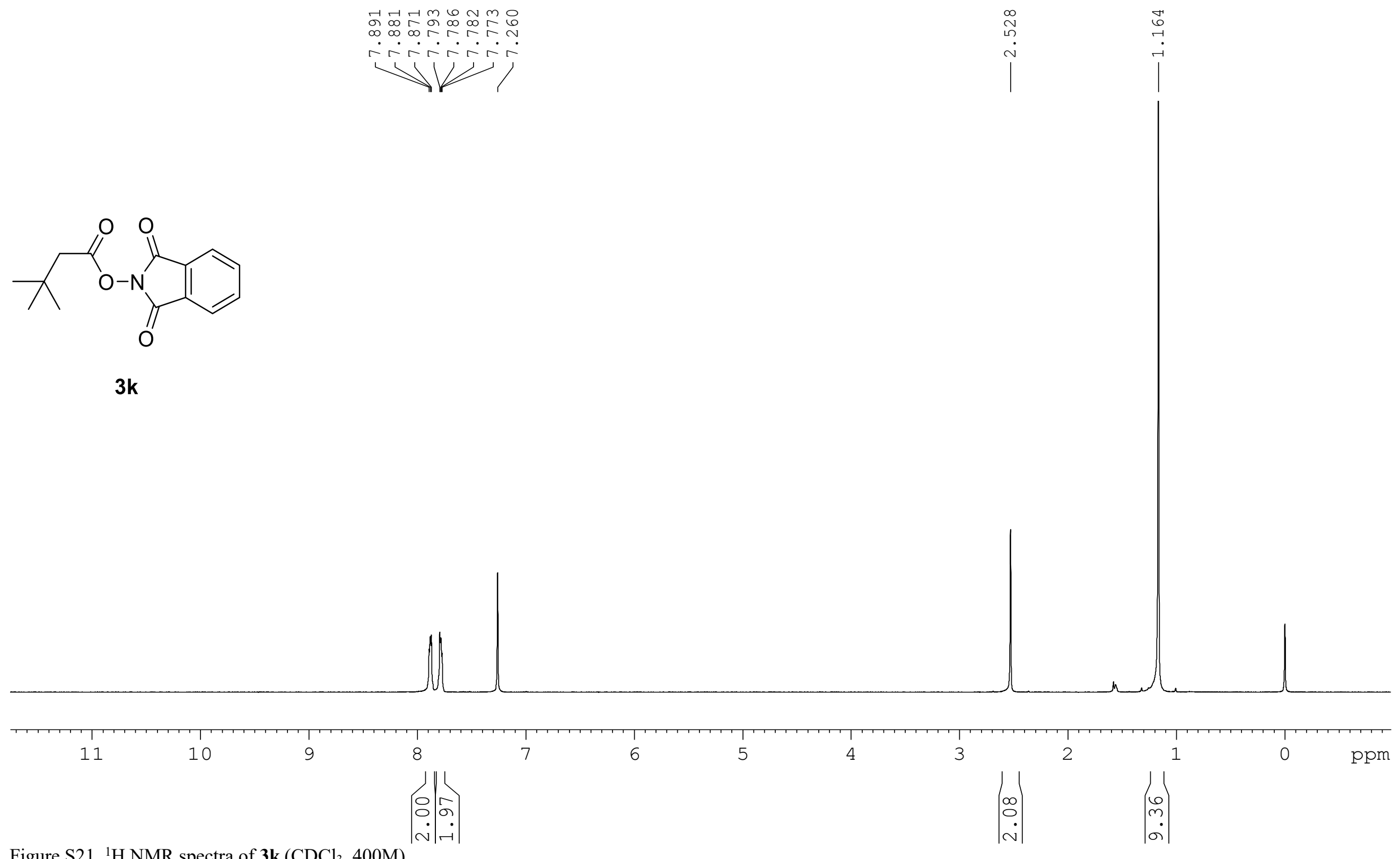

Figure S21. ${ }^{1} \mathrm{H}$ NMR spectra of $\mathbf{3 k}\left(\mathrm{CDCl}_{3}, 400 \mathrm{M}\right)$. 

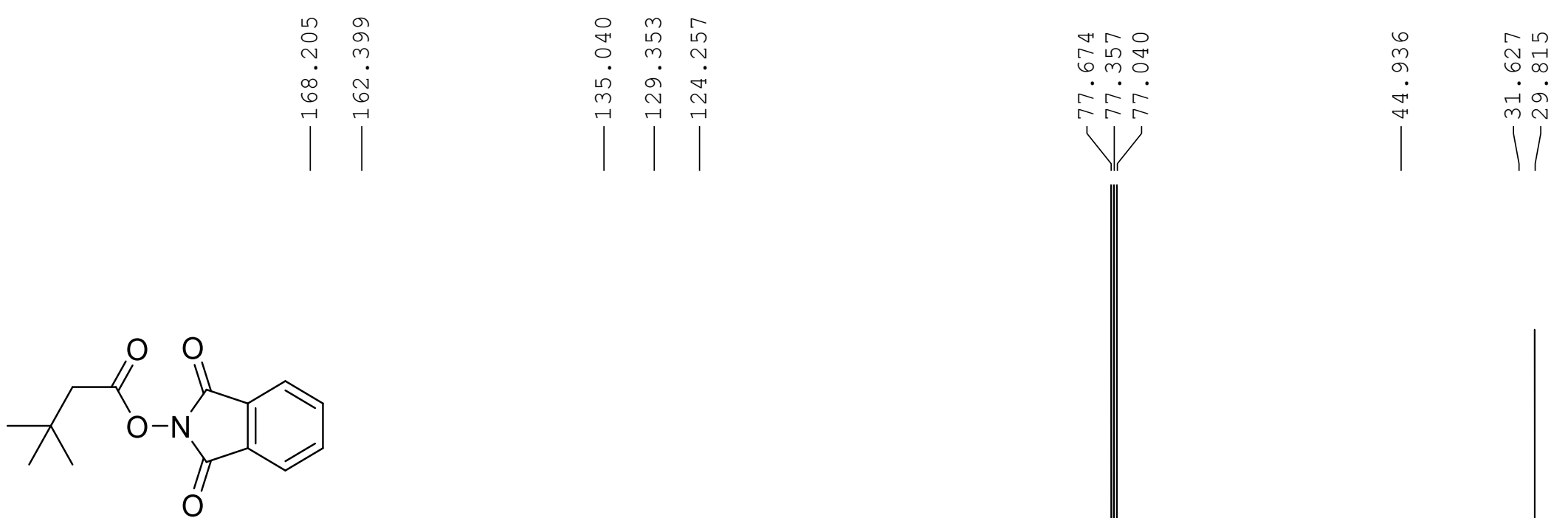

$3 \mathbf{k}$
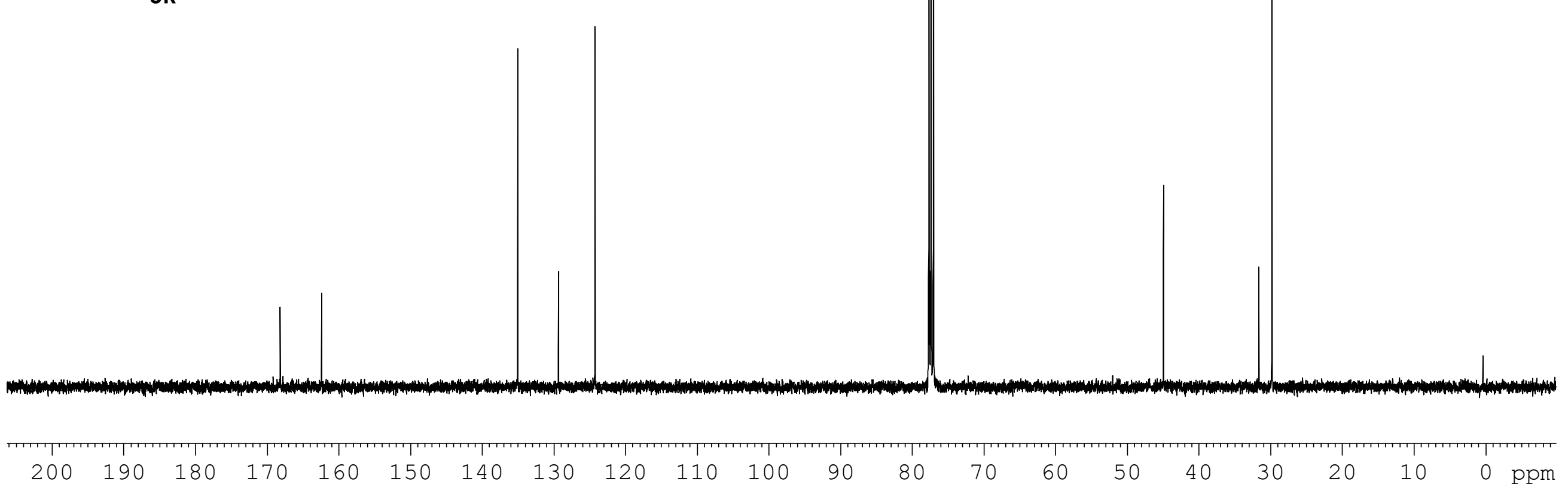
Figure $\mathrm{S} 22 .{ }^{13} \mathrm{C}$ NMR spectra of $\mathbf{3 k}\left(\mathrm{CDCl}_{3}, 100 \mathrm{M}\right)$. 

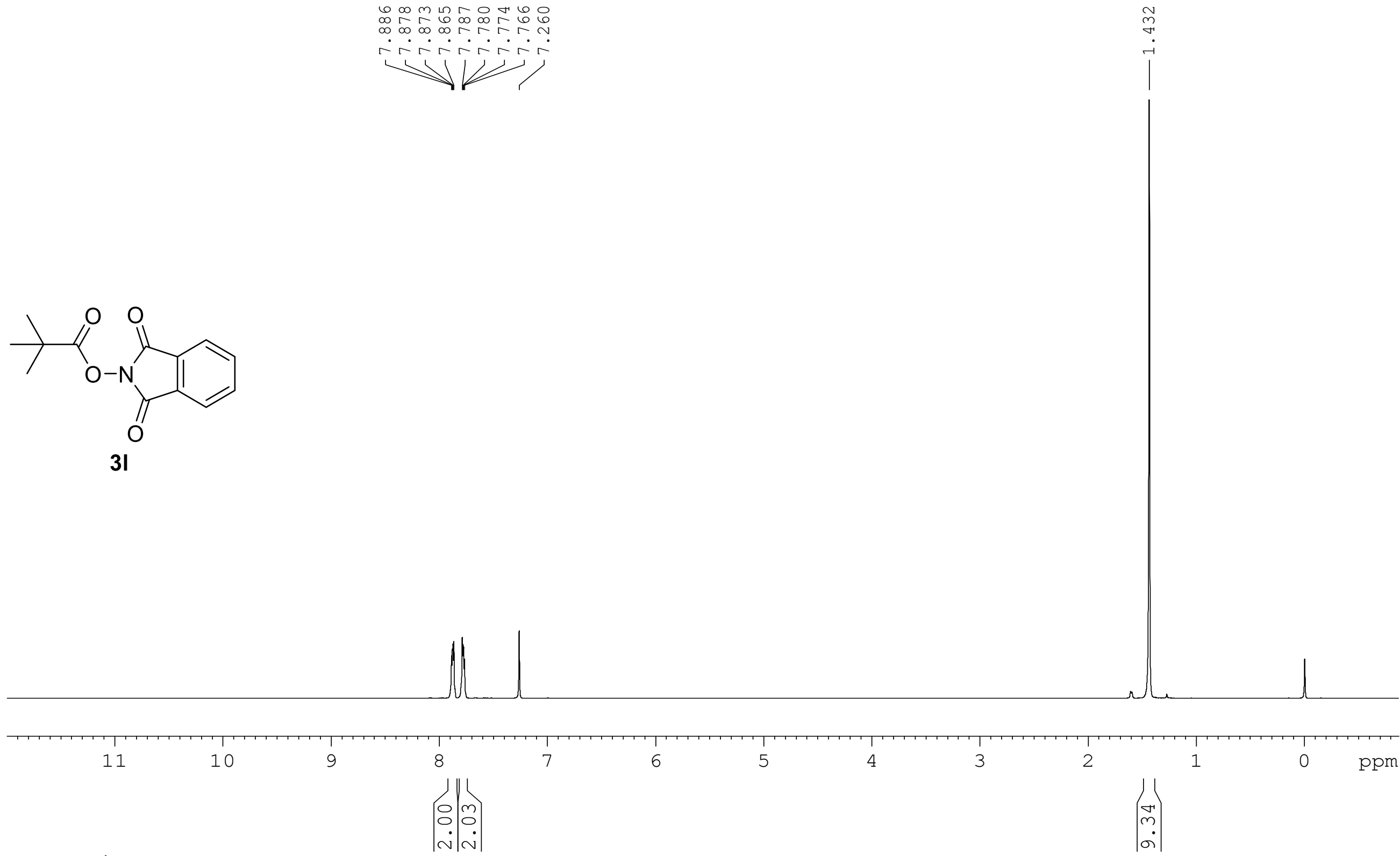

Figure S23. ${ }^{1} \mathrm{H}$ NMR spectra of $\mathbf{3 l}\left(\mathrm{CDCl}_{3}, 400 \mathrm{M}\right)$. 


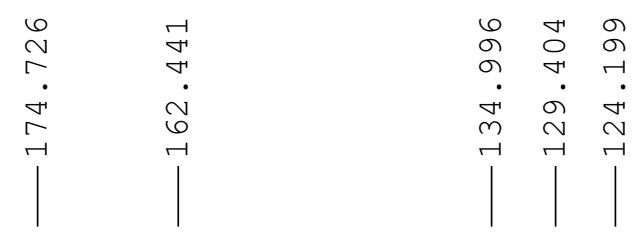
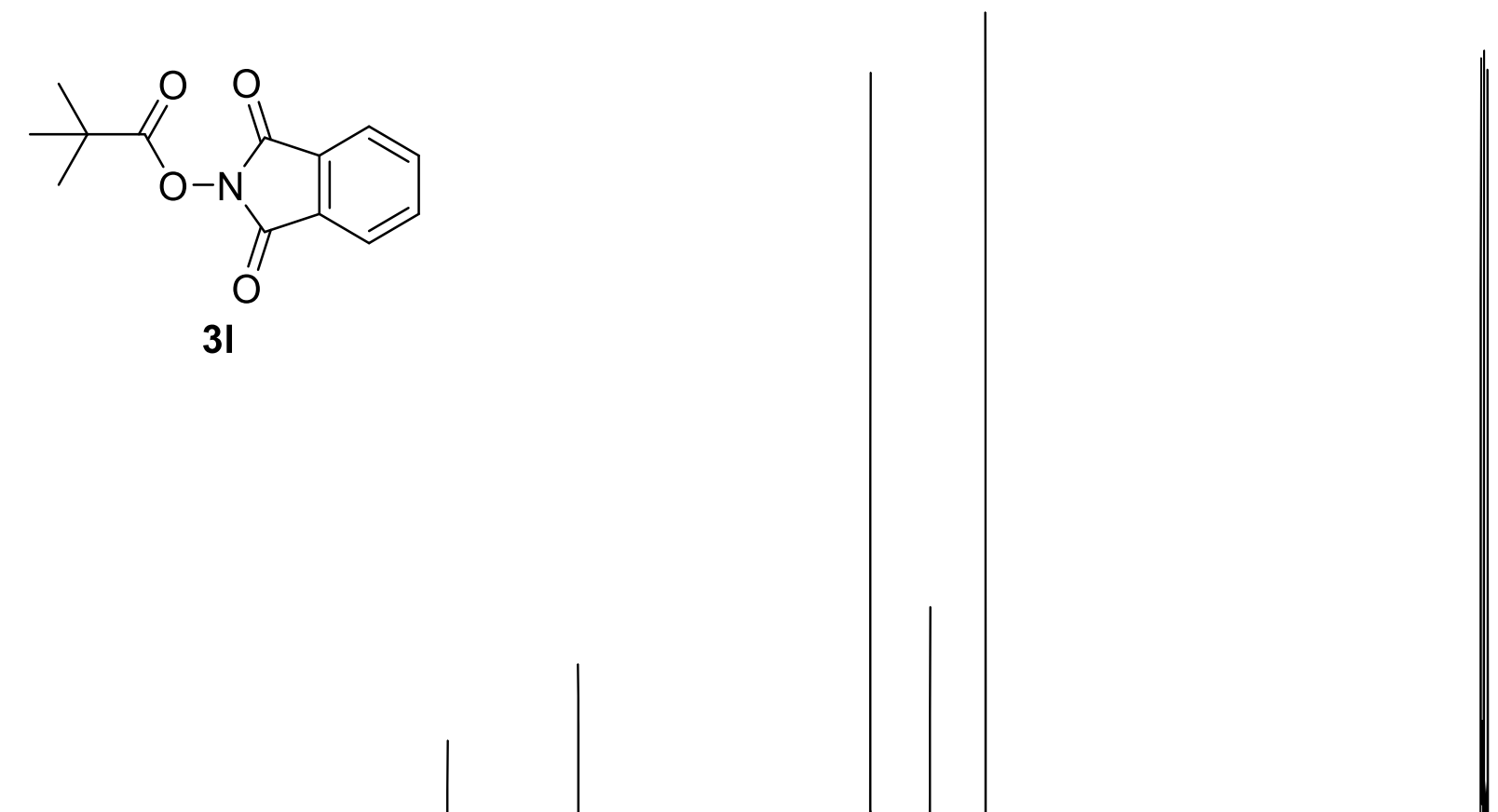

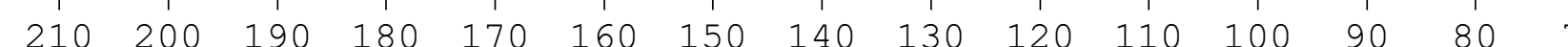
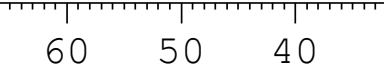

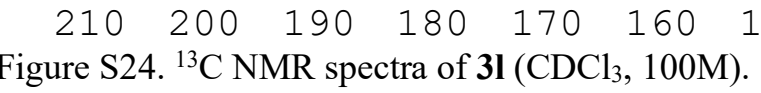




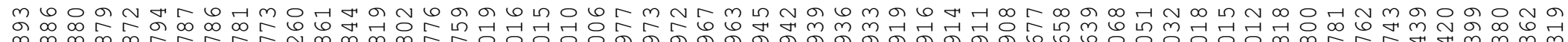

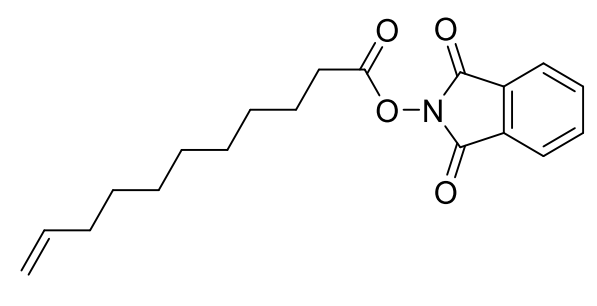

$3 m$

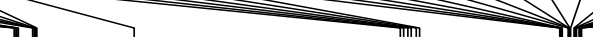

IIIIT

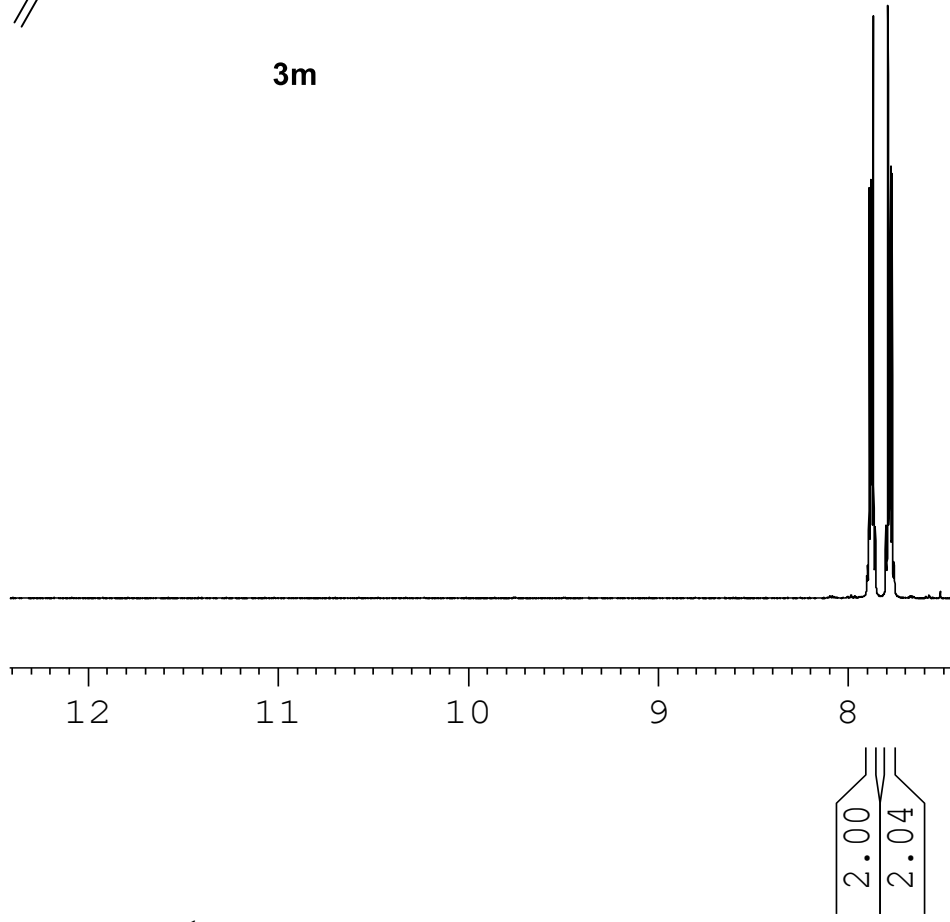

Figure S25. ${ }^{1} \mathrm{H}$ NMR spectra of $\mathbf{3 m}\left(\mathrm{CDCl}_{3}, 400 \mathrm{M}\right)$.

6

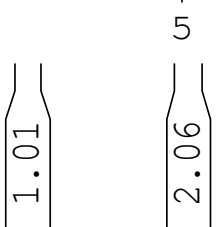

4

3
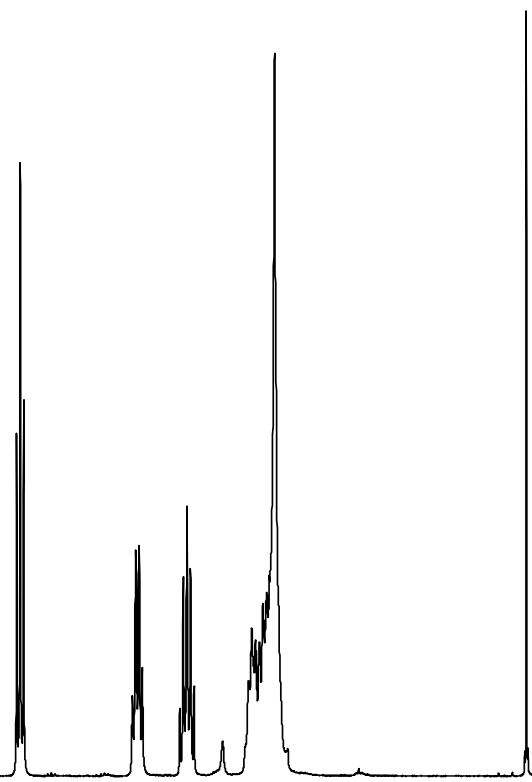

$\left|\begin{array}{c}\mid \\ 0 \\ 0 \\ \sim\end{array}\right|$

1

0

ppm 

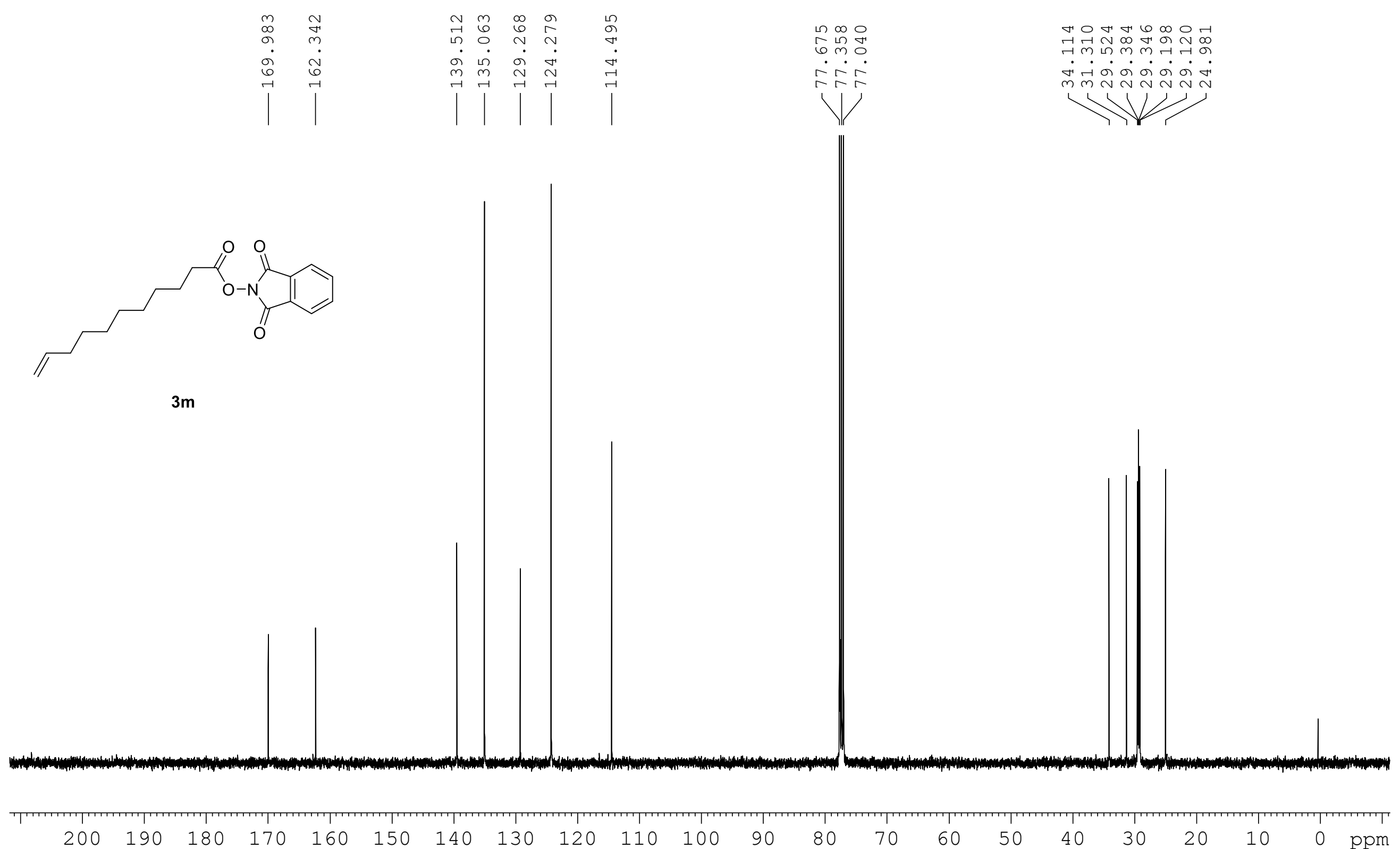

Figure S26. ${ }^{13} \mathrm{C}$ NMR spectra of $\mathbf{3 m}\left(\mathrm{CDCl}_{3}, 100 \mathrm{M}\right)$. 


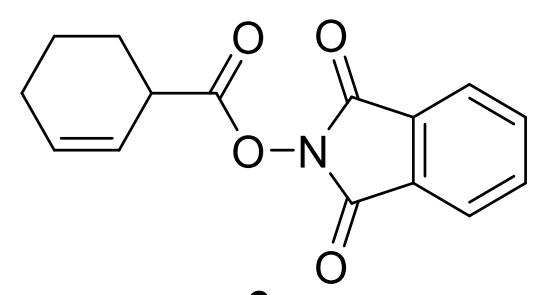

$3 n$

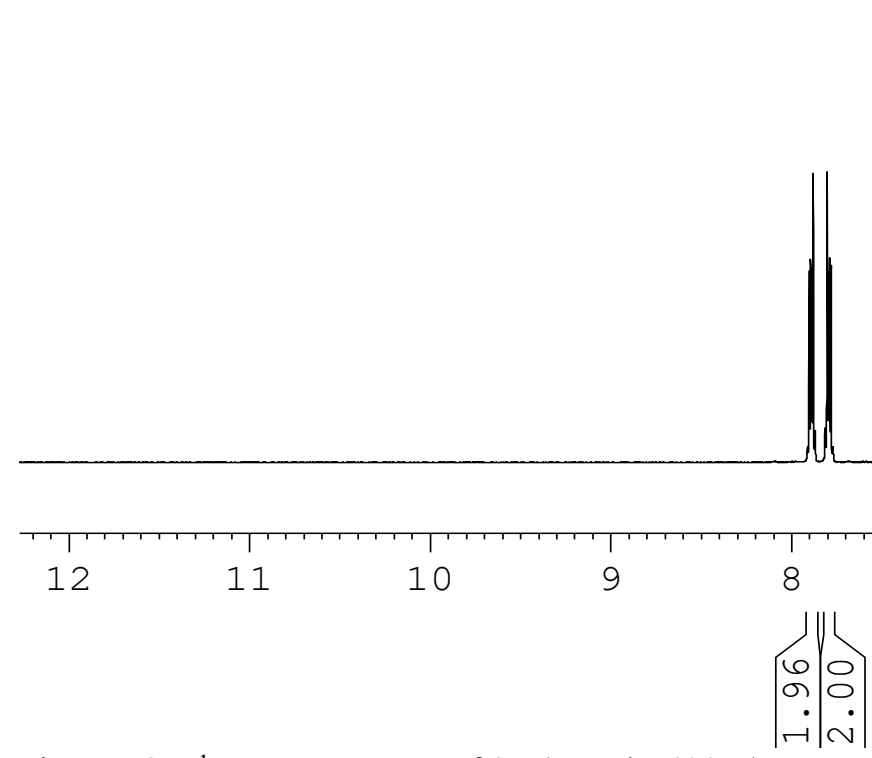

Figure S27. ${ }^{1} \mathrm{H}$ NMR spectra of $\mathbf{3 n}\left(\mathrm{CDCl}_{3}, 400 \mathrm{M}\right)$.

7

6

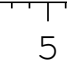

4

(2)

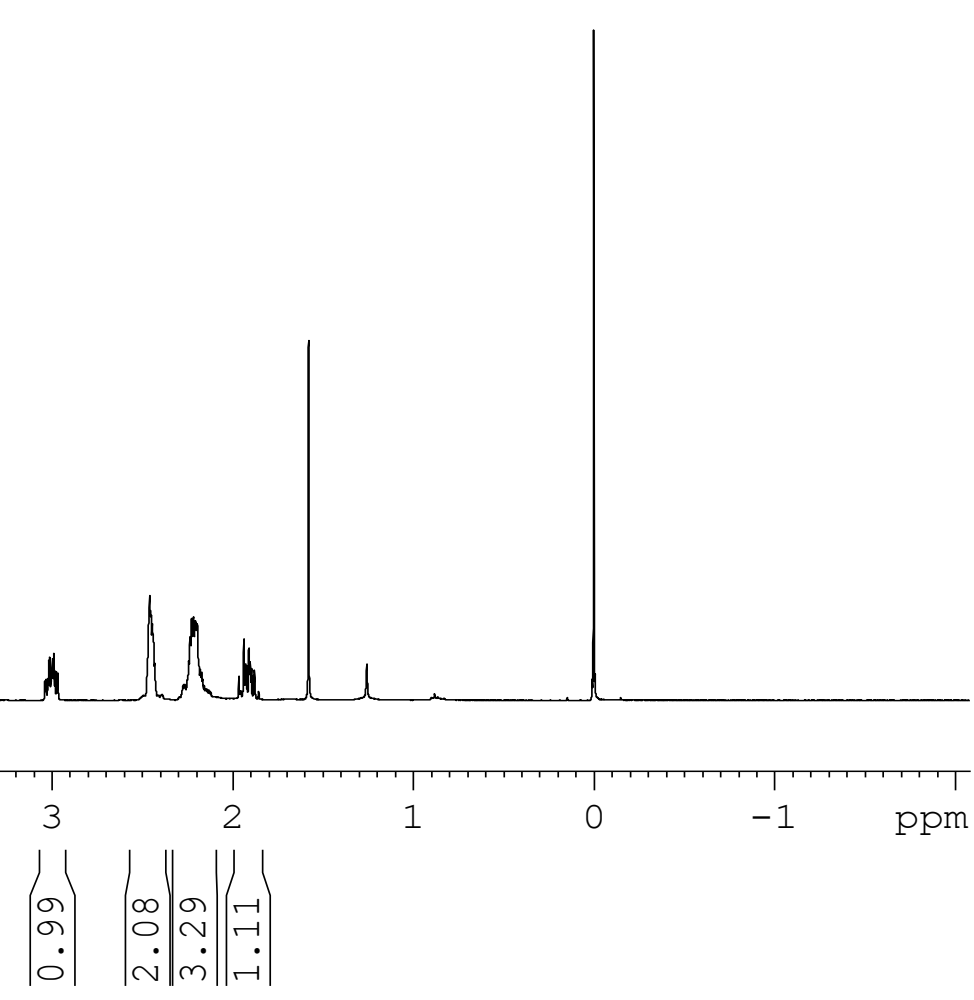



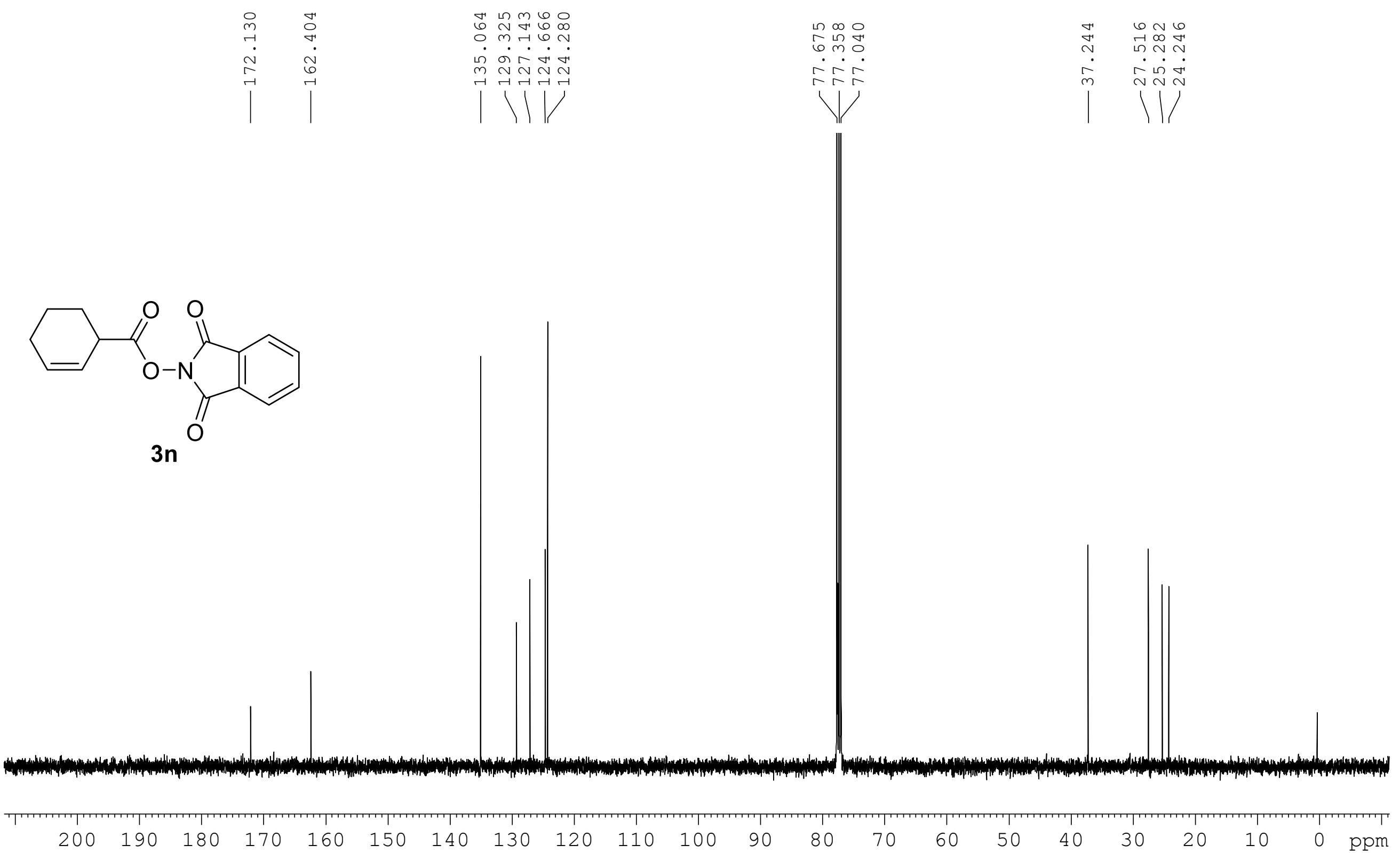

Figure S28. ${ }^{13} \mathrm{C}$ NMR spectra of $\mathbf{3 n}\left(\mathrm{CDCl}_{3}, 100 \mathrm{M}\right)$. 


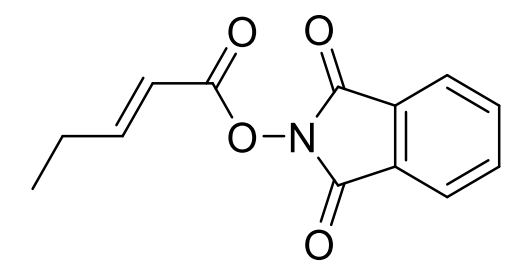

30

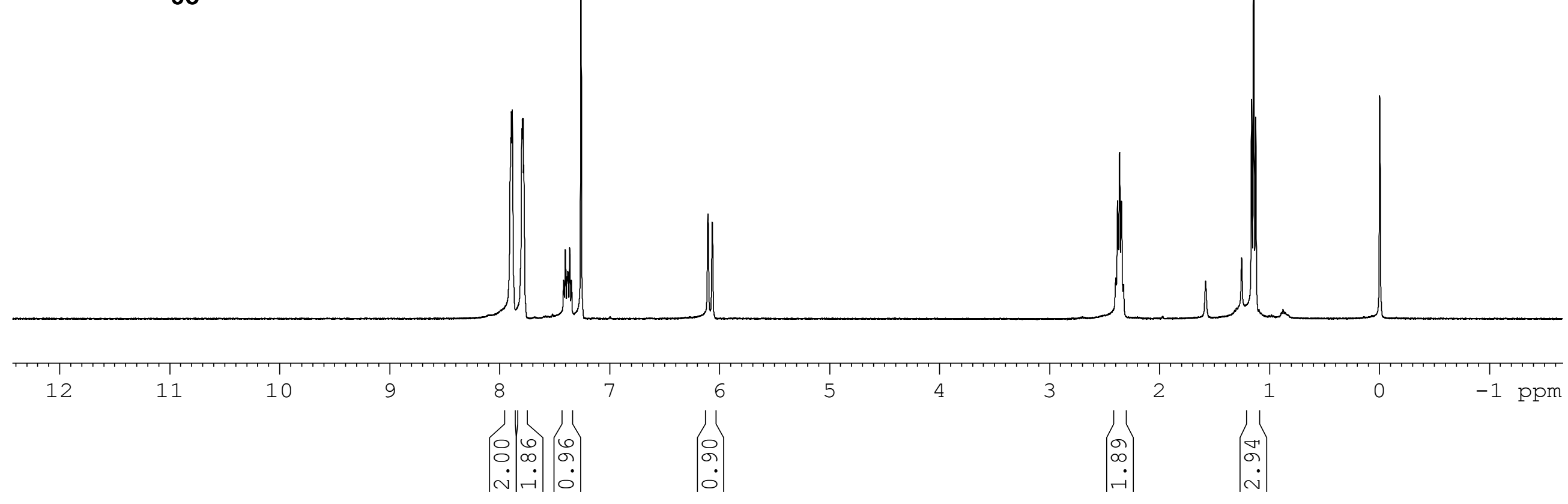

Figure S29. ${ }^{1} \mathrm{H}$ NMR spectra of $\mathbf{3 o}\left(\mathrm{CDCl}_{3}, 400 \mathrm{M}\right)$. 


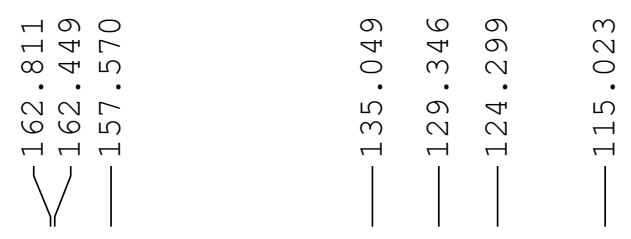

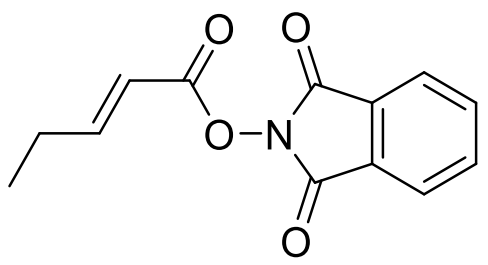

30
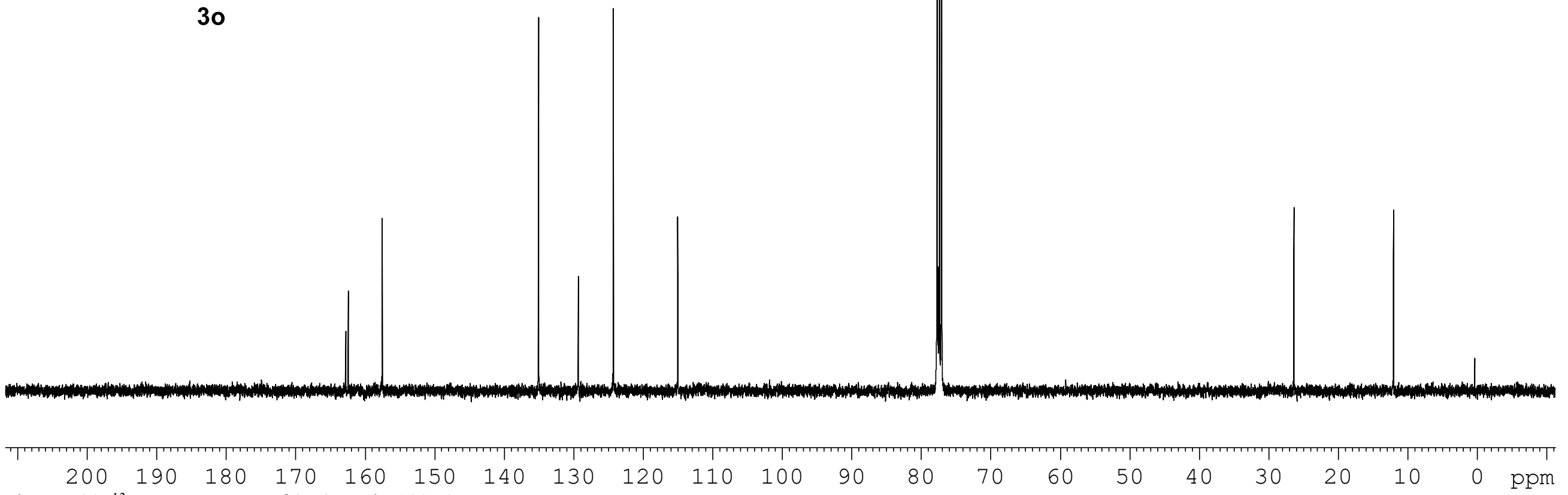

$40 \quad 30$

20

Figure S30. ${ }^{13} \mathrm{C}$ NMR spectra of $\mathbf{3 o}\left(\mathrm{CDCl}_{3}, 100 \mathrm{M}\right)$. 


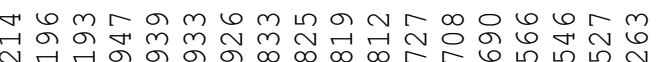

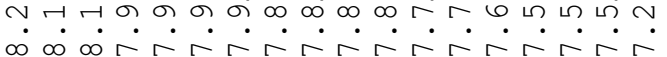<smiles>O=C(ON1C(=O)c2ccccc2C1=O)c1ccccc1</smiles>

$3 p$

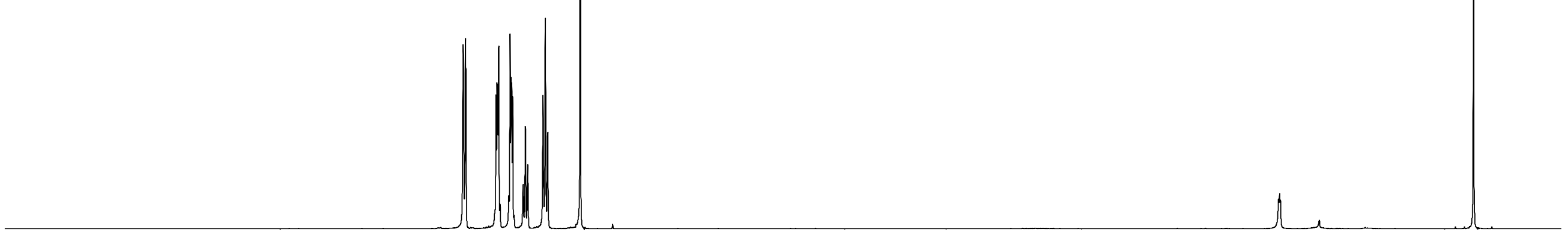

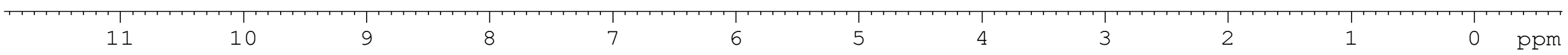

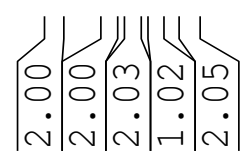

Figure S31. ${ }^{1} \mathrm{H}$ NMR spectra of $3 \mathbf{p}\left(\mathrm{CDCl}_{3}, 400 \mathrm{M}\right)$.

S-49 

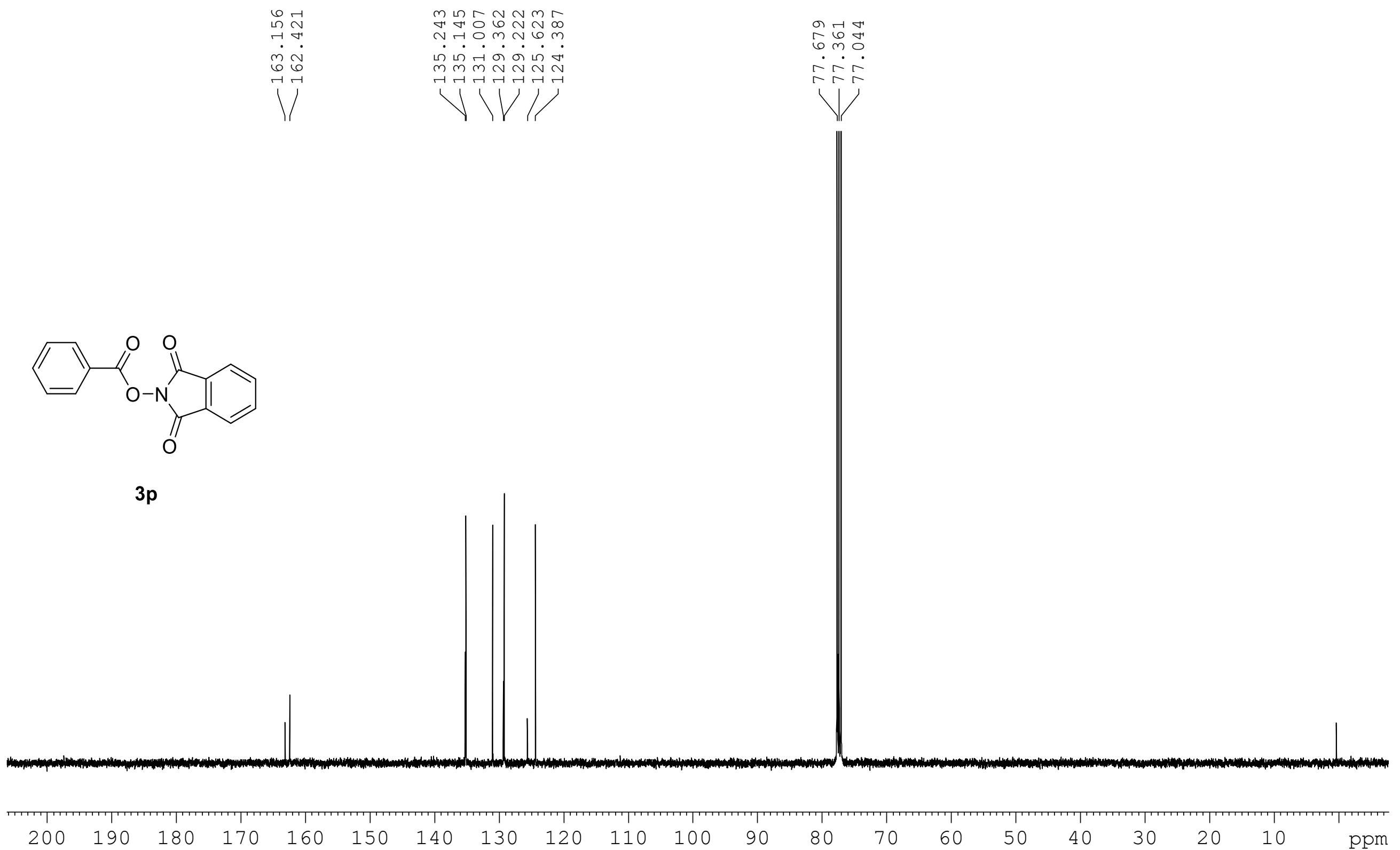

Figure $\mathrm{S} 32 .{ }^{13} \mathrm{C}$ NMR spectra of $\mathbf{3 p}\left(\mathrm{CDCl}_{3}, 100 \mathrm{M}\right)$. 
๙

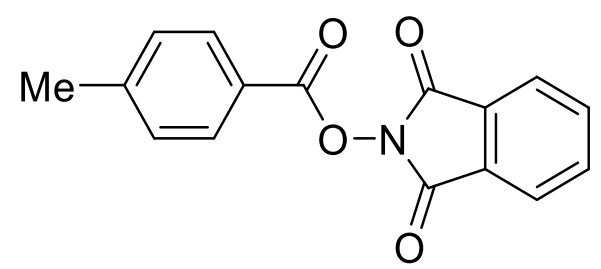

$3 q$

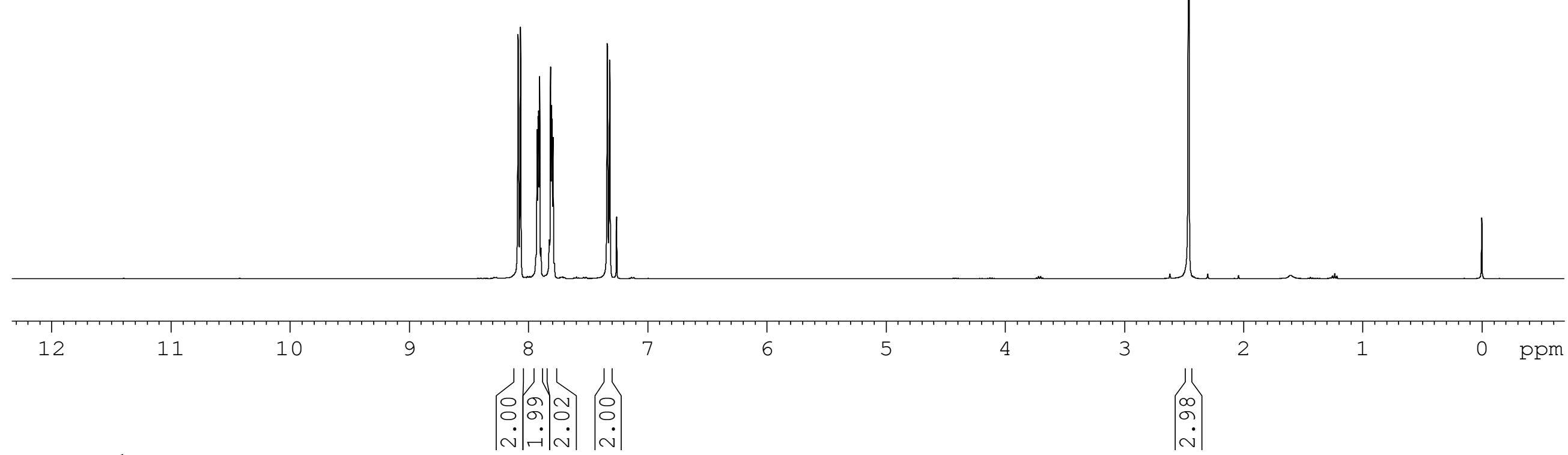

Figure S33. ${ }^{1} \mathrm{H}$ NMR spectra of $\mathbf{3 q}\left(\mathrm{CDCl}_{3}, 400 \mathrm{M}\right)$. 


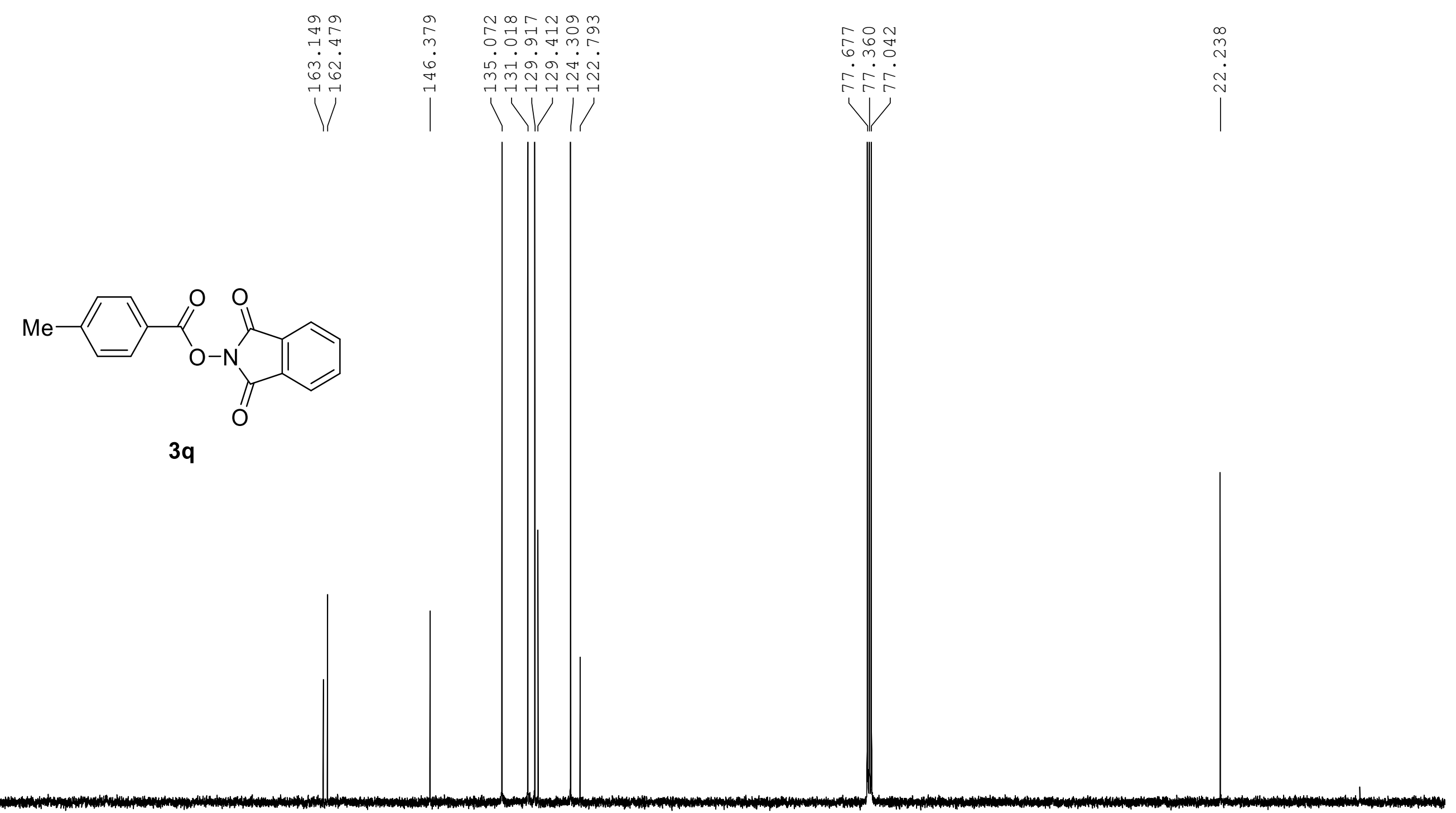

Figure S34. ${ }^{13} \mathrm{C}$ NMR spectra of $\mathbf{3 q}\left(\mathrm{CDCl}_{3}, 100 \mathrm{M}\right)$. 


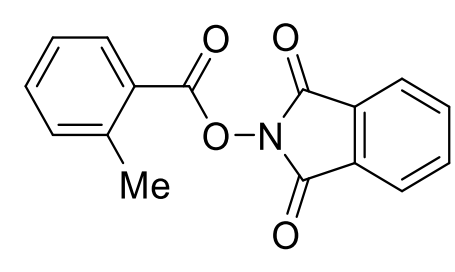

$3 r$

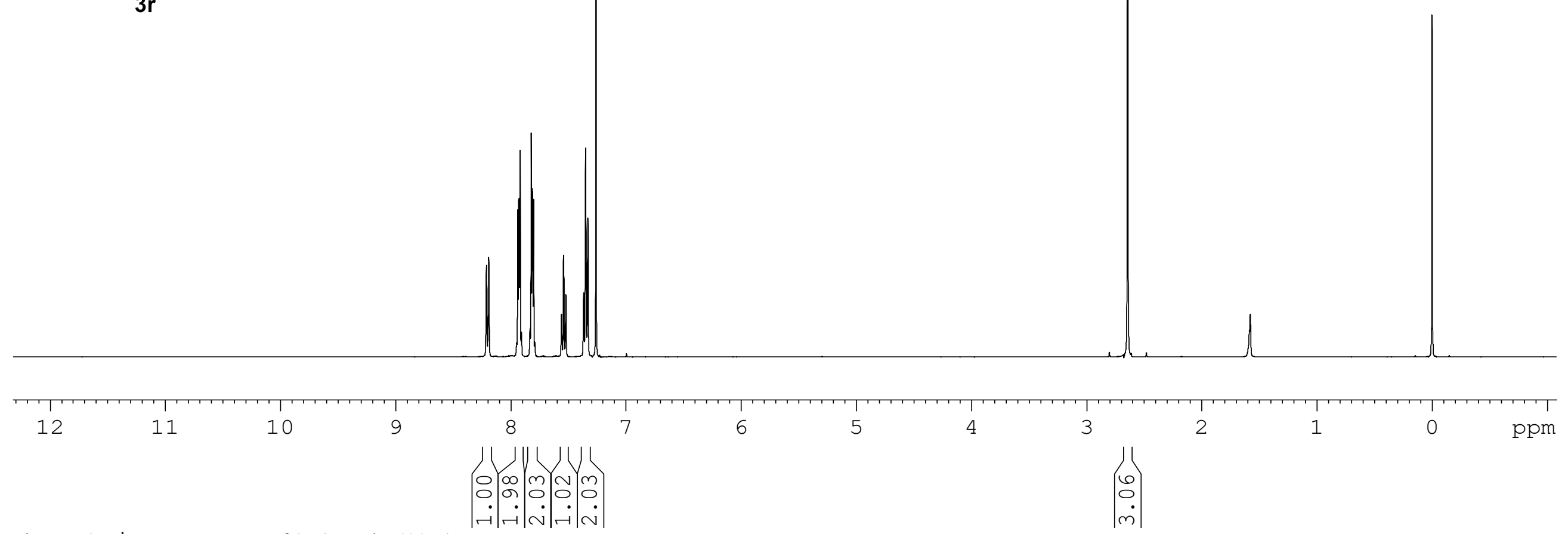

Figure $\mathrm{S} 35 .{ }^{1} \mathrm{H}$ NMR spectra of $\mathbf{3 r}\left(\mathrm{CDCl}_{3}, 400 \mathrm{M}\right)$. 


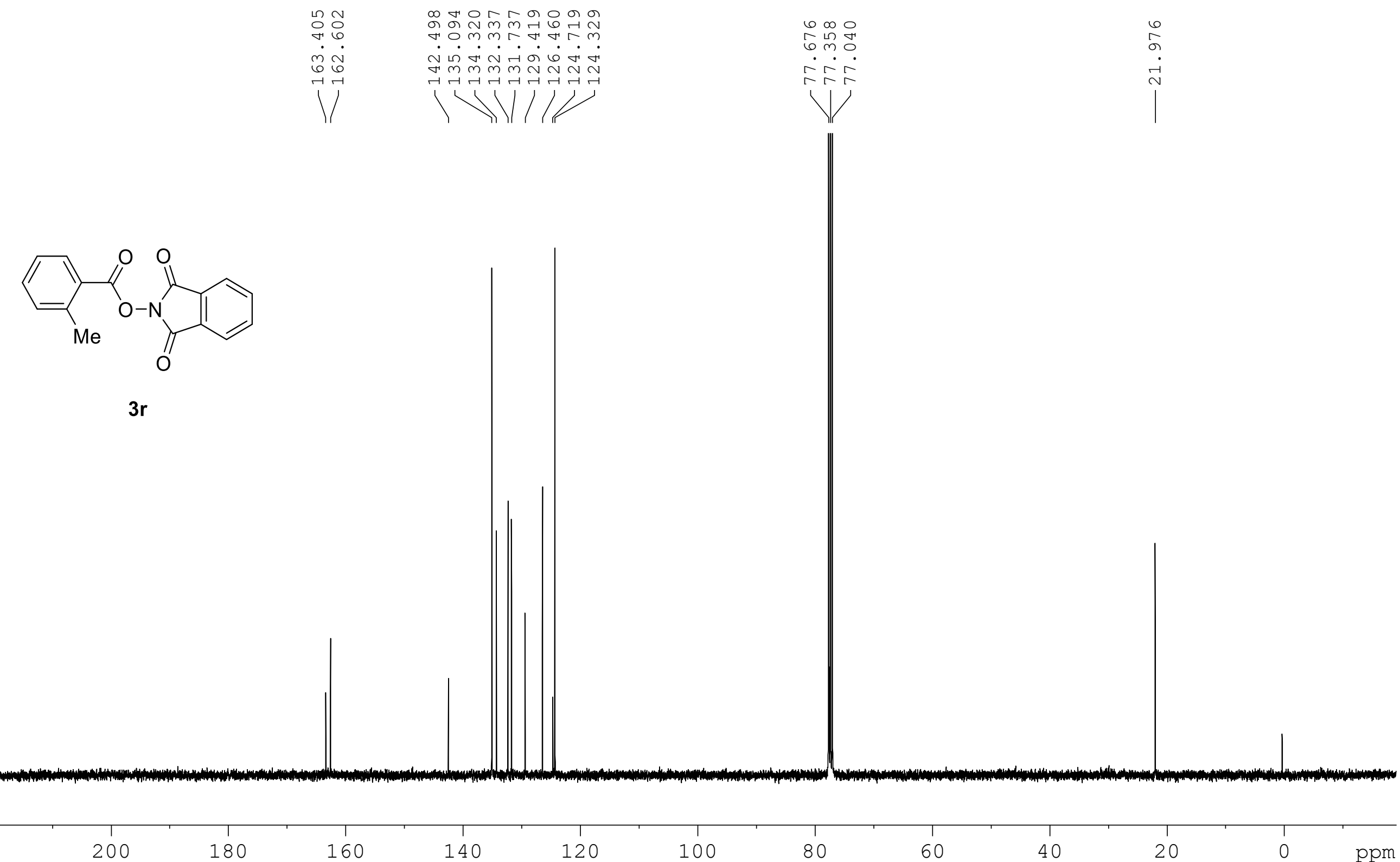

Figure S36. ${ }^{13} \mathrm{C}$ NMR spectra of $3 \mathbf{r}\left(\mathrm{CDCl}_{3}, 100 \mathrm{M}\right)$. 


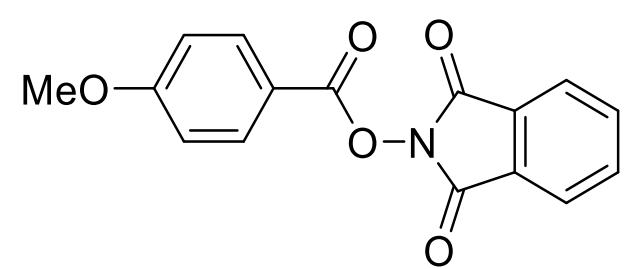

3s

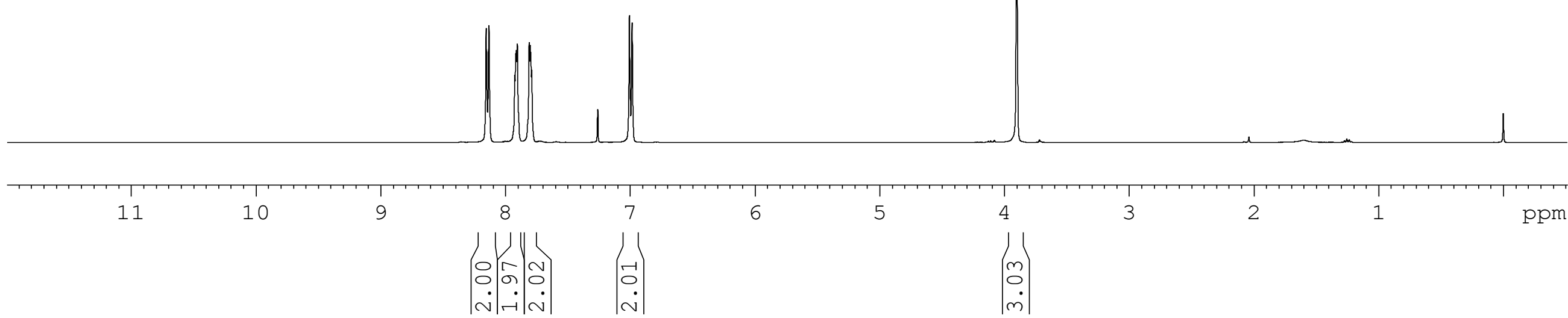

Figure S37. ${ }^{1} \mathrm{H}$ NMR spectra of $3 \mathbf{s}\left(\mathrm{CDCl}_{3}, 400 \mathrm{M}\right)$. 

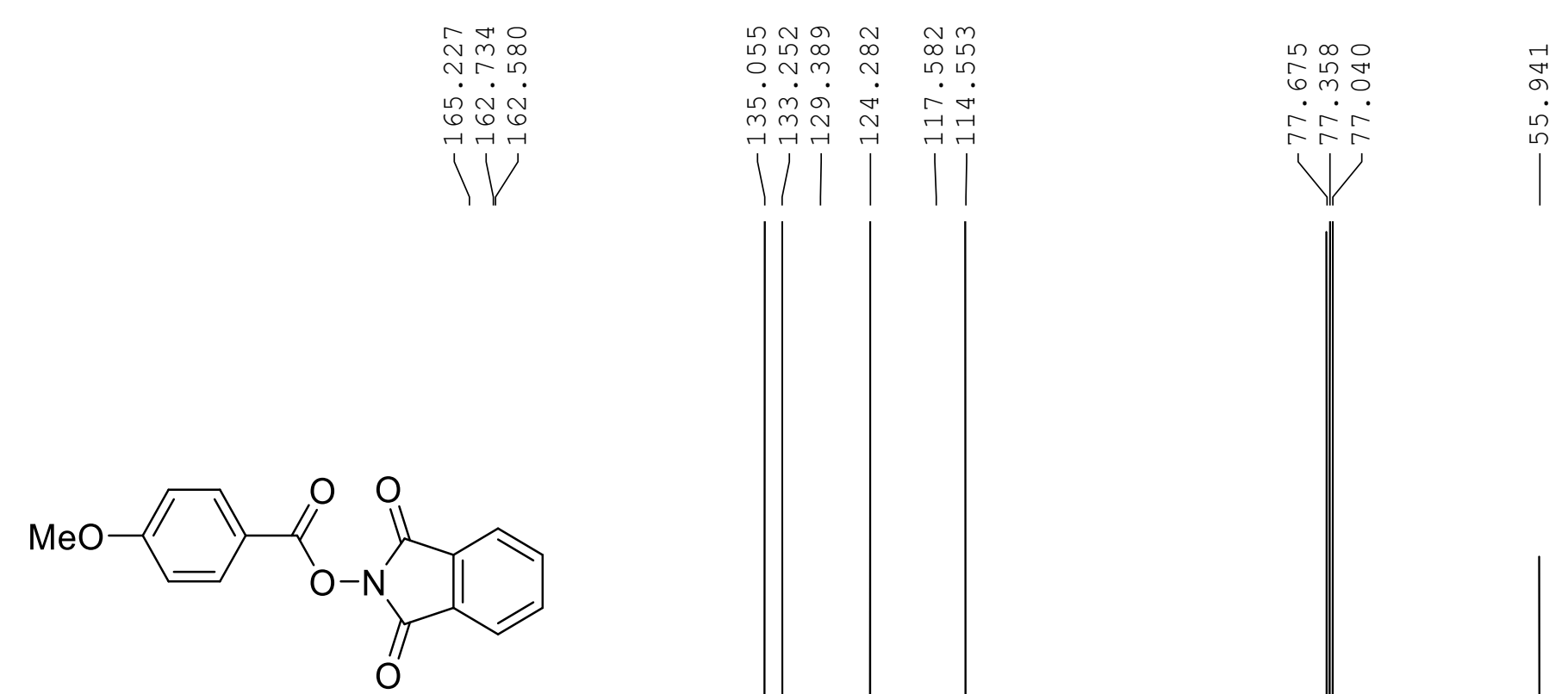

$3 s$
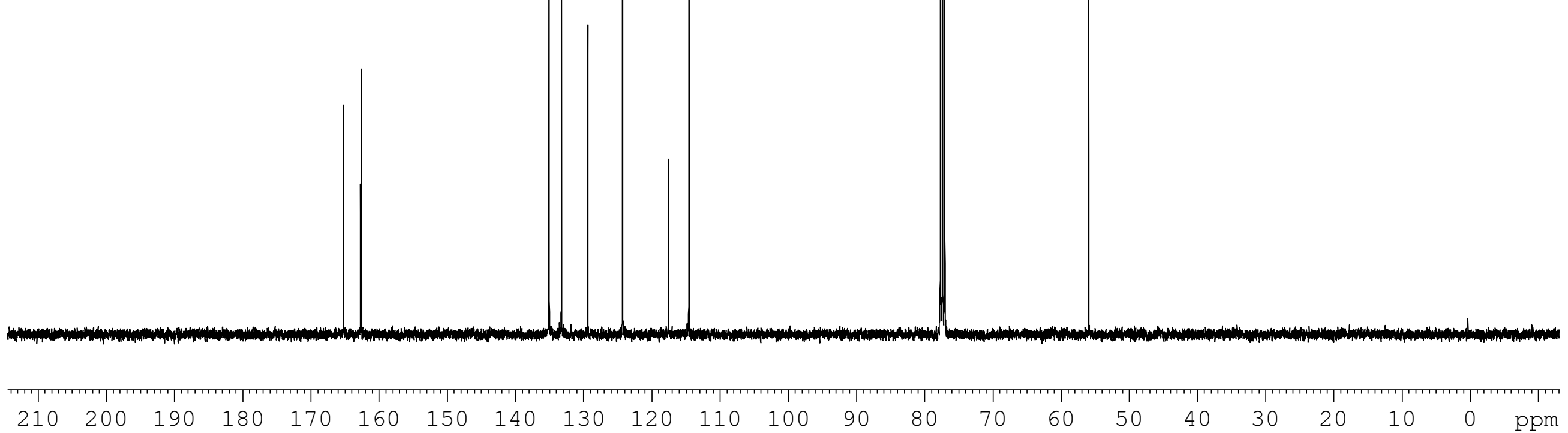

Figure $\mathrm{S} 38 .{ }^{13} \mathrm{C}$ NMR spectra of $\mathbf{3 s}\left(\mathrm{CDCl}_{3}, 100 \mathrm{M}\right)$. 


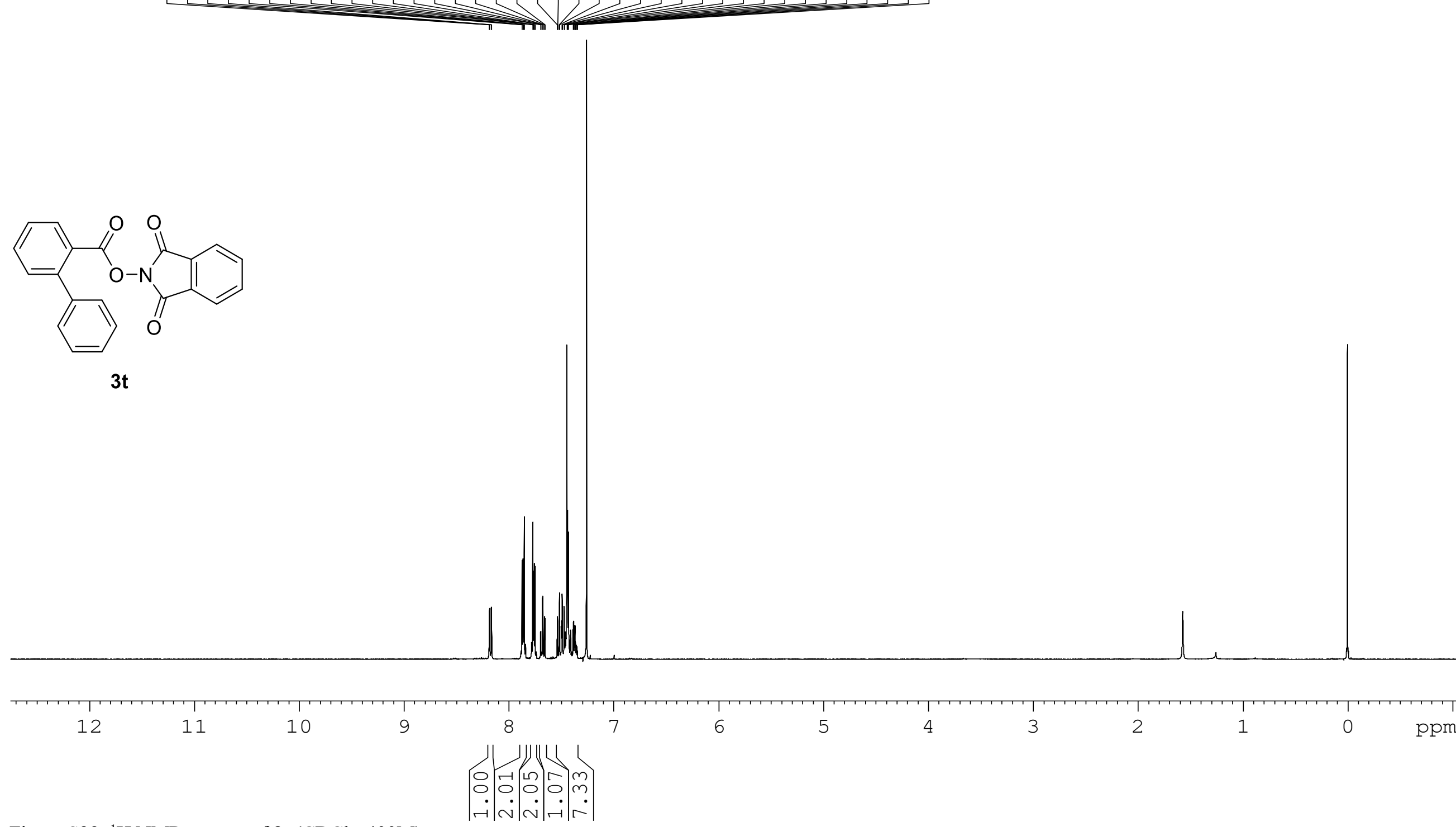

Figure S39. ${ }^{1} \mathrm{H}$ NMR spectra of $\mathbf{3 t}\left(\mathrm{CDCl}_{3}, 400 \mathrm{M}\right)$. 

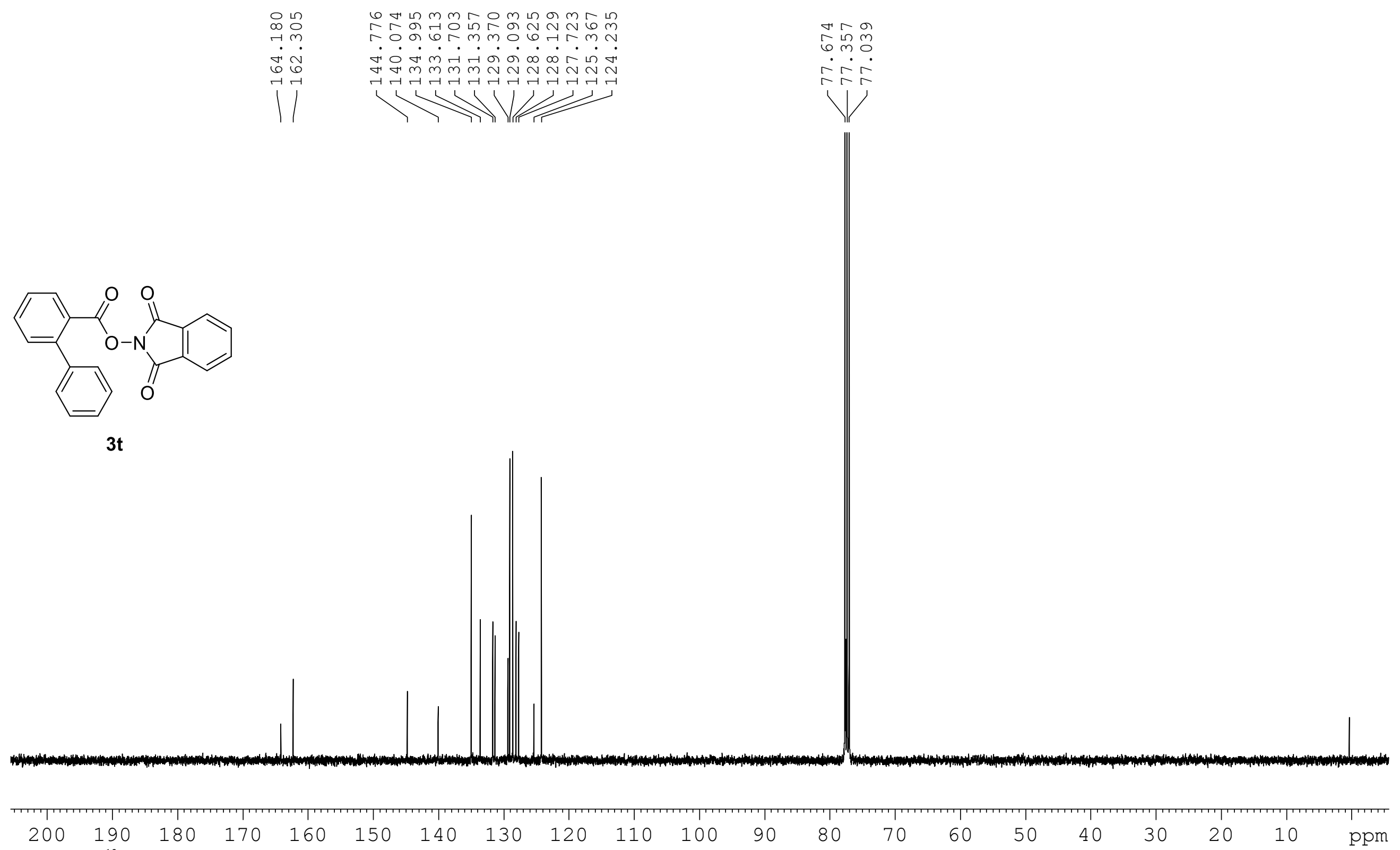

Figure $\mathrm{S} 40 .{ }^{13} \mathrm{C}$ NMR spectra of $\mathbf{3 t}\left(\mathrm{CDCl}_{3}, 100 \mathrm{M}\right)$. 


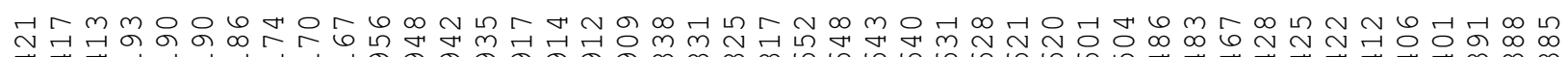

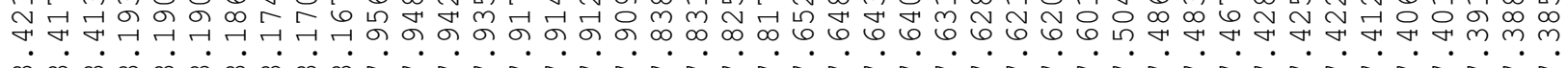

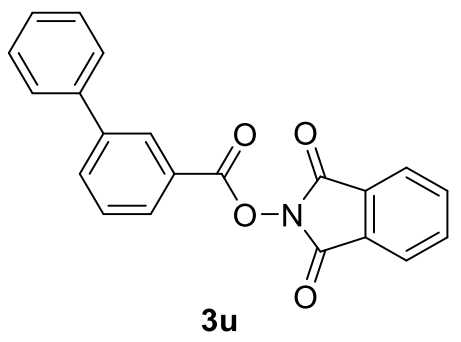
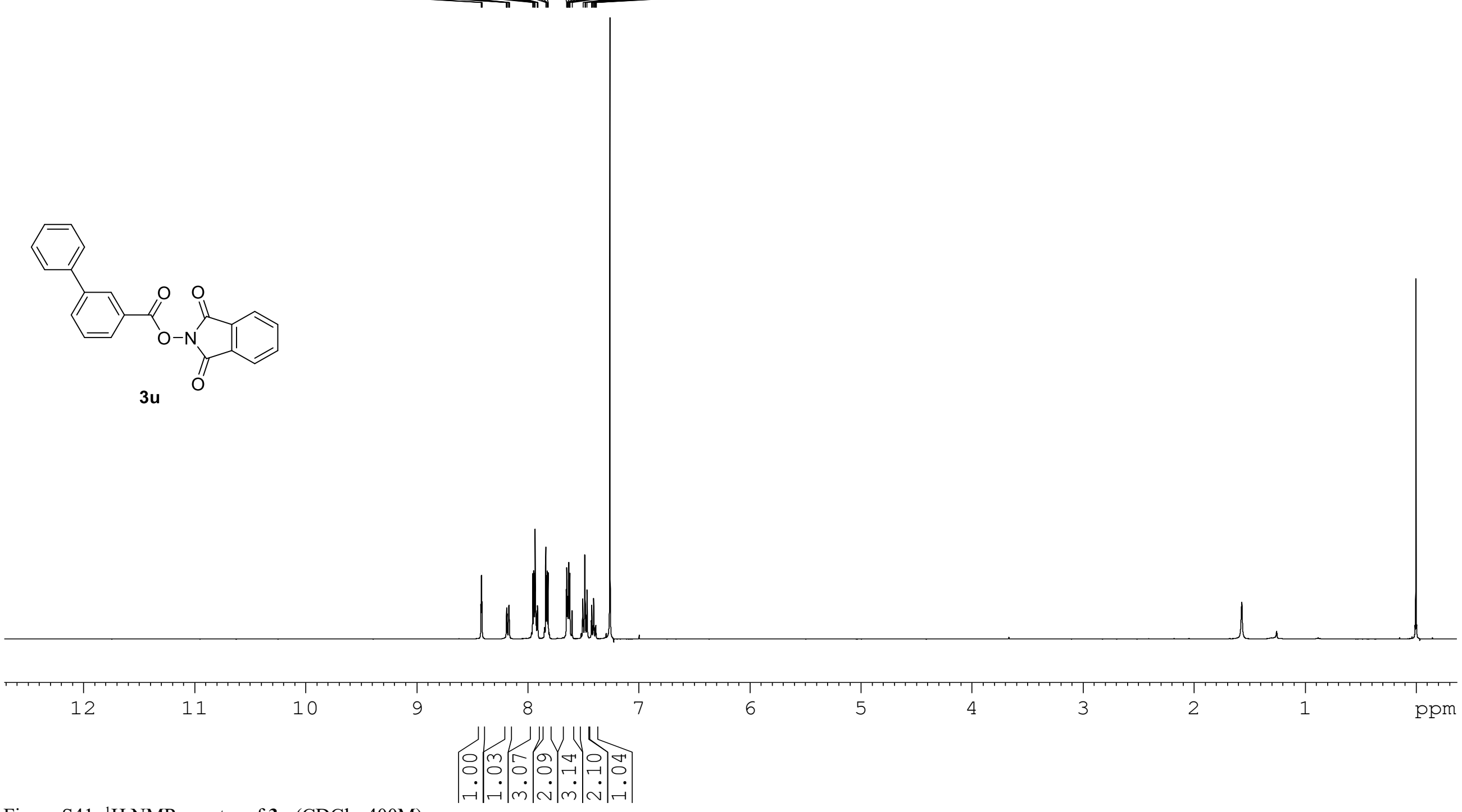

Figure S41. ${ }^{1} \mathrm{H}$ NMR spectra of $\mathbf{3} \mathbf{u}\left(\mathrm{CDCl}_{3}, 400 \mathrm{M}\right)$.

S-59 


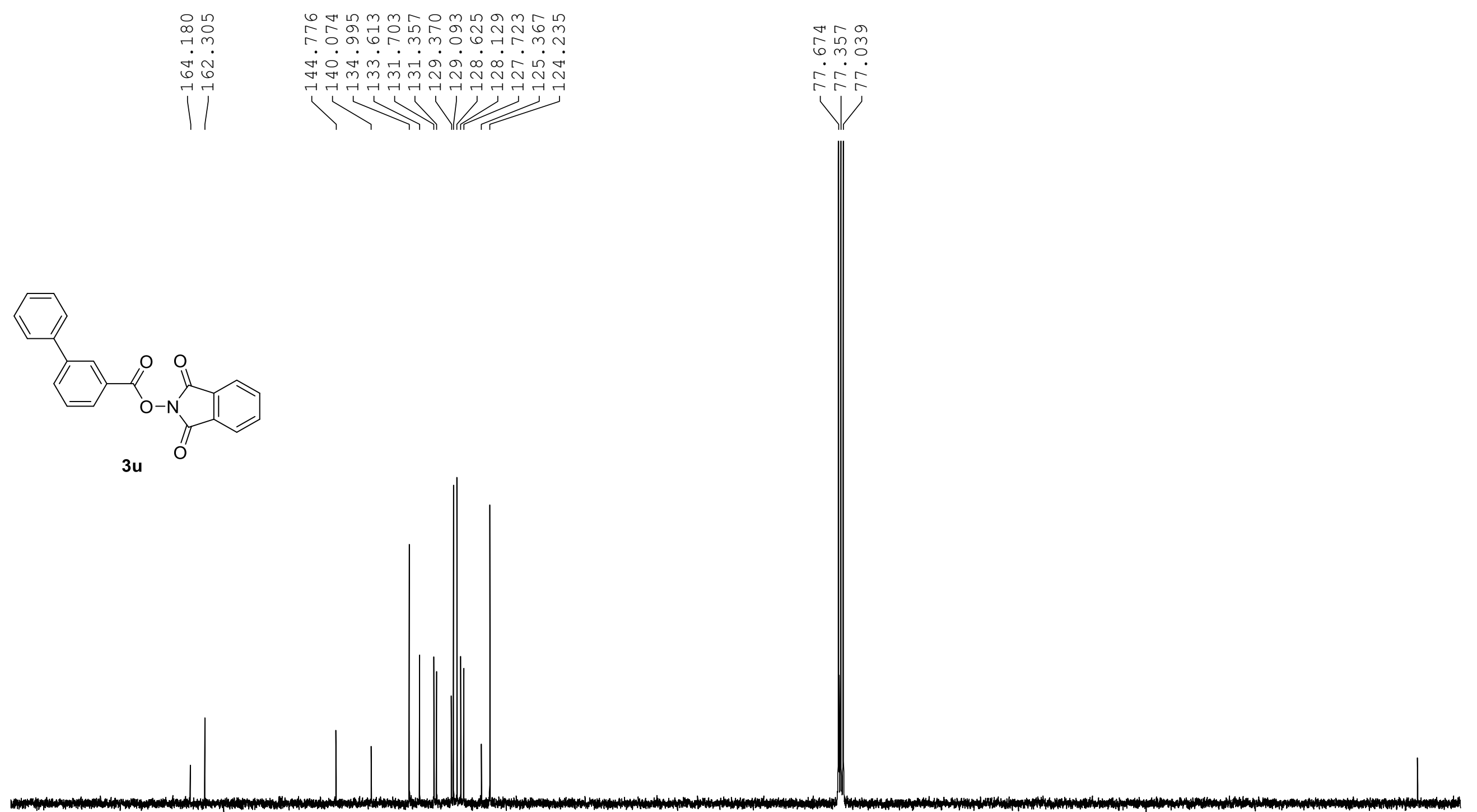

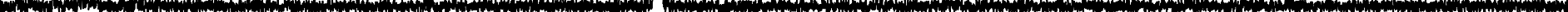

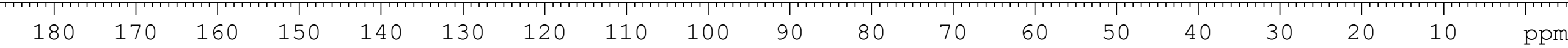

Figure $\mathrm{S} 42 .{ }^{13} \mathrm{C}$ NMR spectra of $3 \mathbf{u}\left(\mathrm{CDCl}_{3}, 100 \mathrm{M}\right)$. 


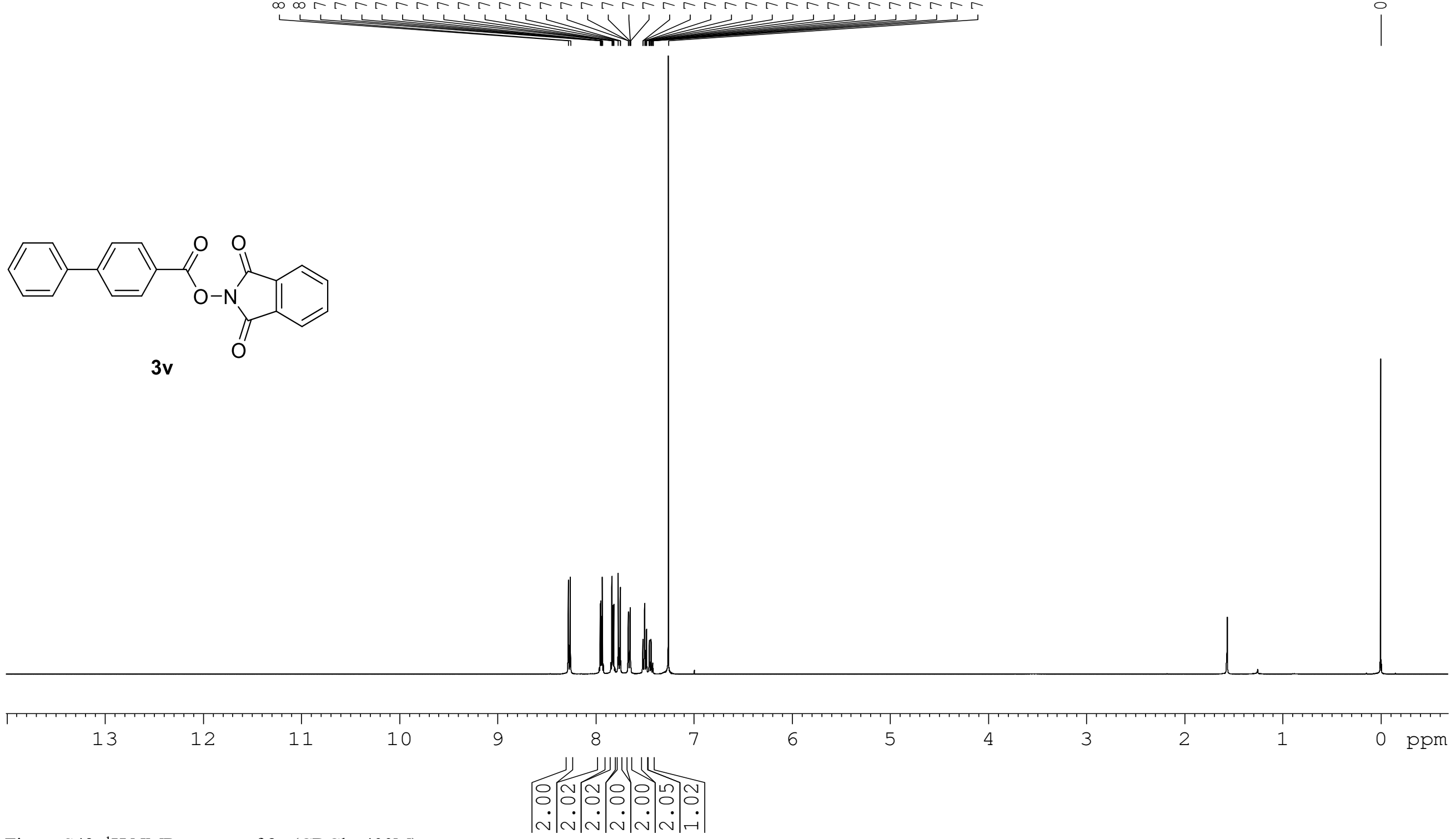

Figure $\mathrm{S} 43 .{ }^{1} \mathrm{H}$ NMR spectra of $\mathbf{3 v}\left(\mathrm{CDCl}_{3}, 400 \mathrm{M}\right)$. 

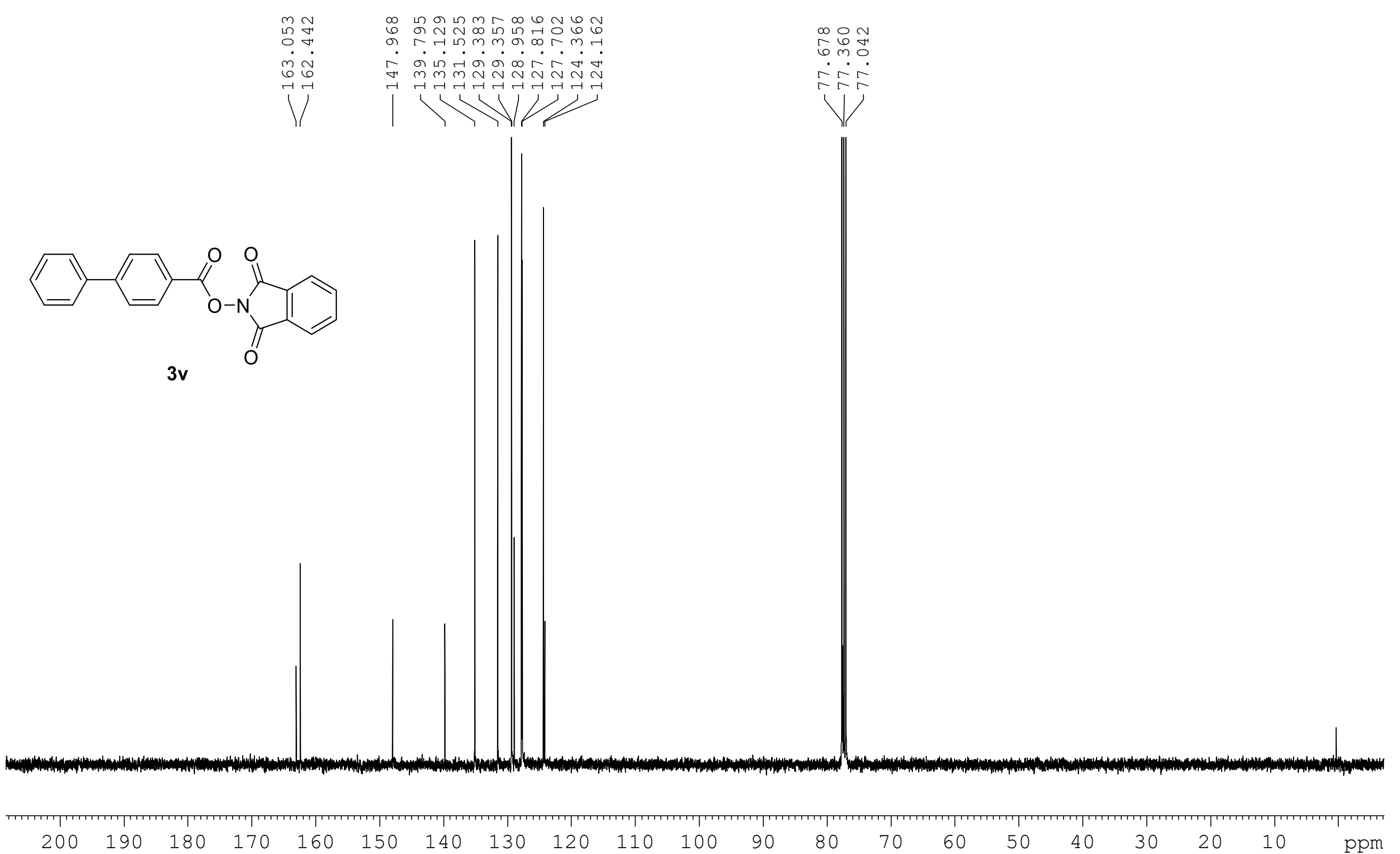

Figure S44. ${ }^{13} \mathrm{C}$ NMR spectra of $\mathbf{3 v}\left(\mathrm{CDCl}_{3}, 100 \mathrm{M}\right)$. 


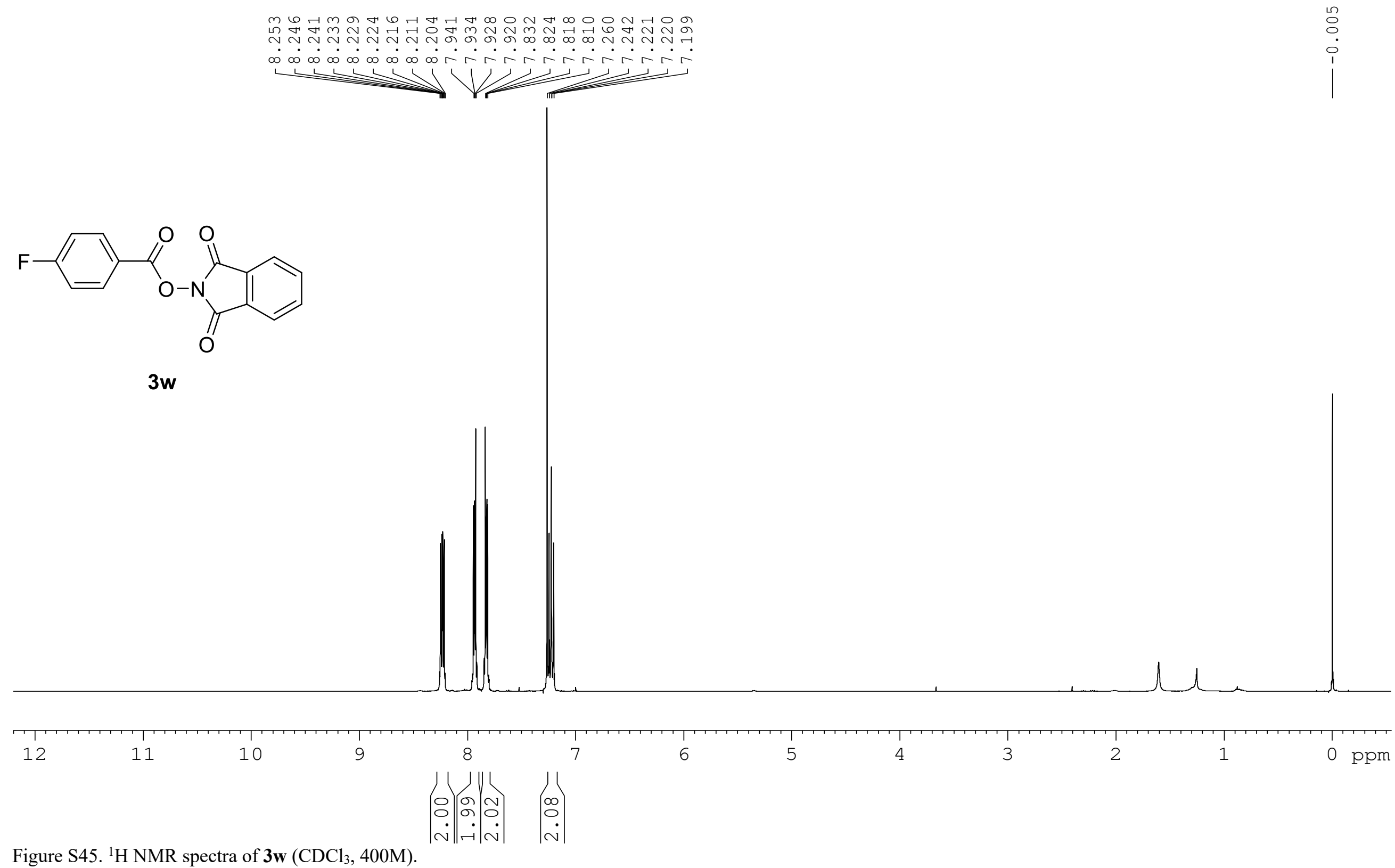

Figure S45. ${ }^{1} \mathrm{H}$ NMR spectra of $\mathbf{3 w}\left(\mathrm{CDCl}_{3}, 400 \mathrm{M}\right)$ 


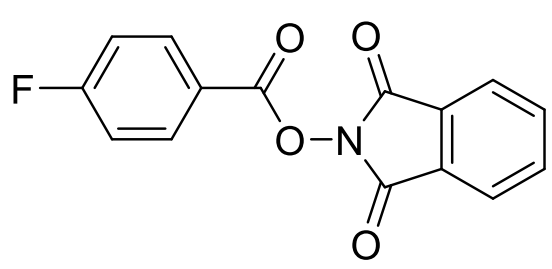

$3 w$

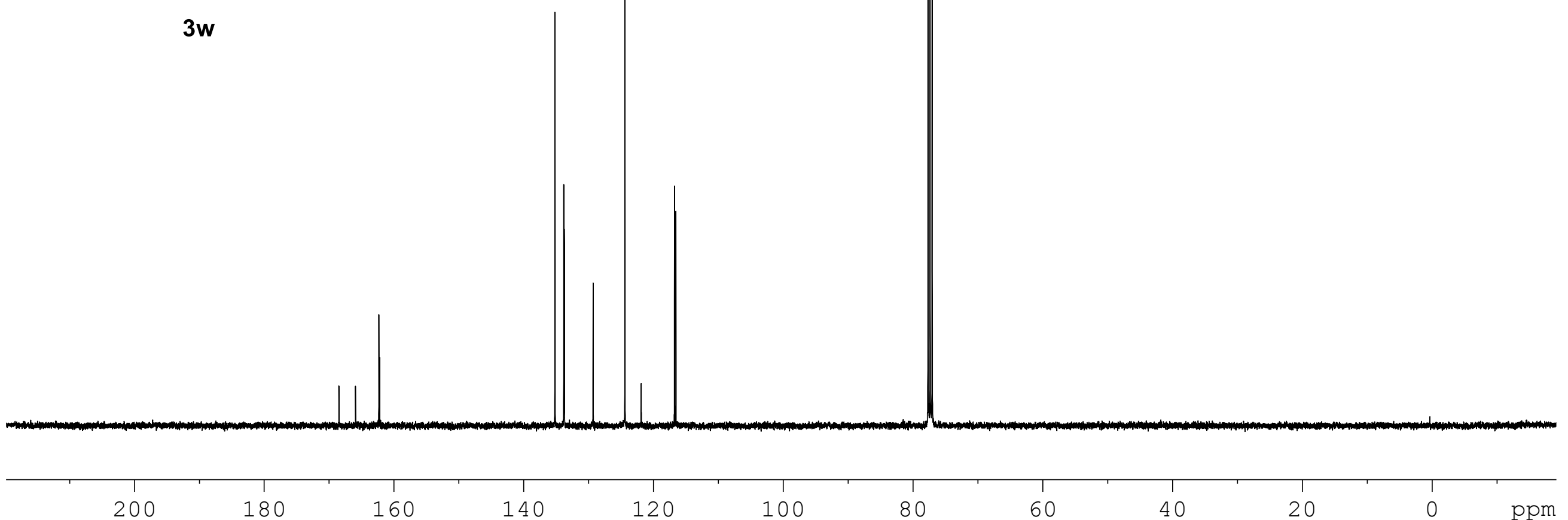

Figure S46. ${ }^{13} \mathrm{C}$ NMR spectra of $\mathbf{3 w}\left(\mathrm{CDCl}_{3}, 100 \mathrm{M}\right)$. 

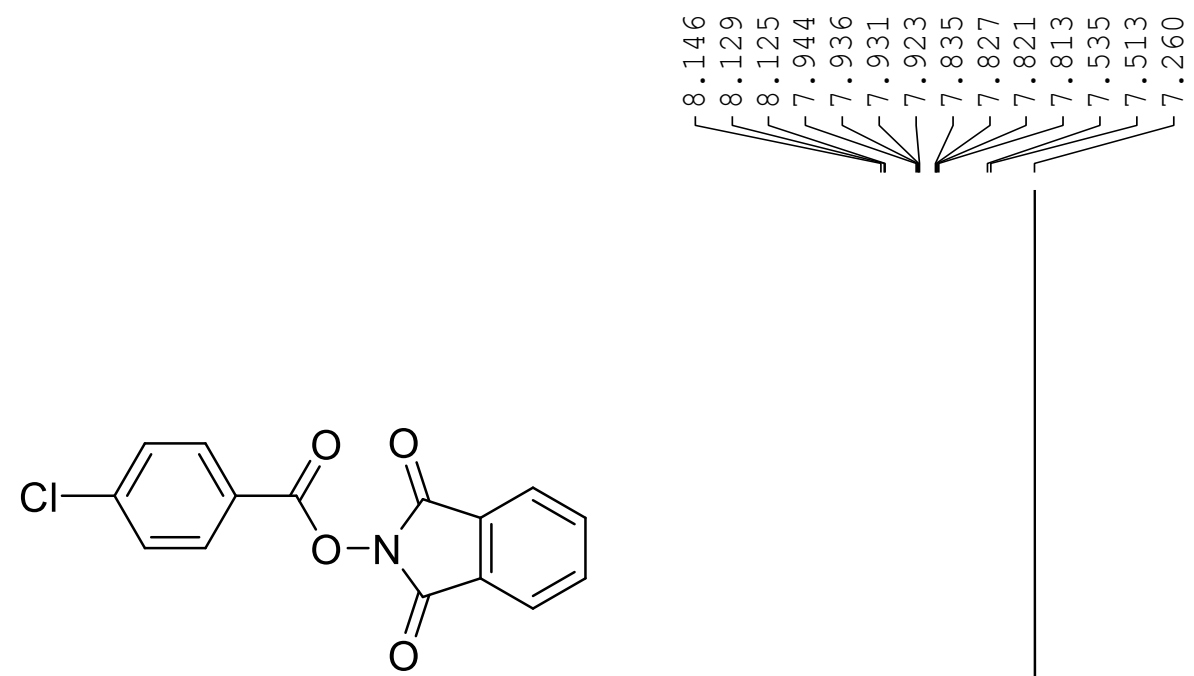

$3 x$

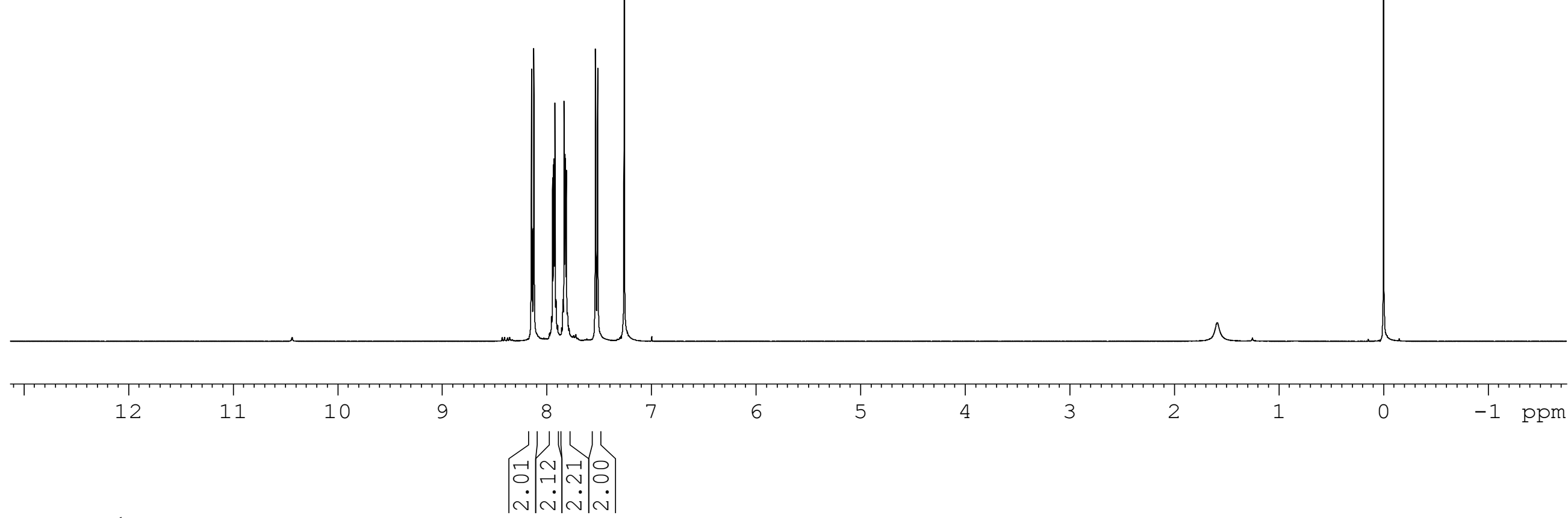

Figure S47. ${ }^{1} \mathrm{H}$ NMR spectra of $\mathbf{3 x}\left(\mathrm{CDCl}_{3}, 400 \mathrm{M}\right)$. 


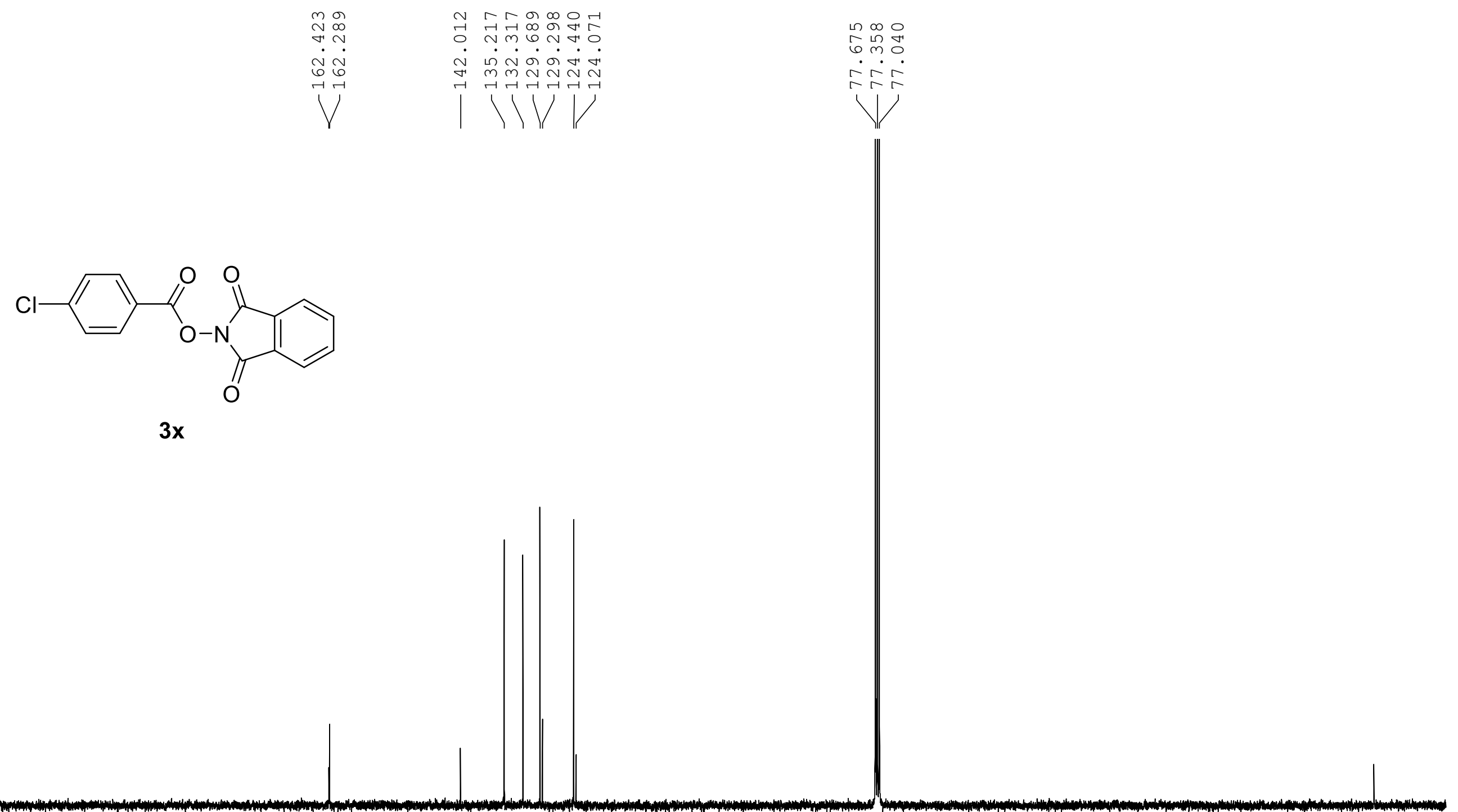

Figure $\mathrm{S} 48 .{ }^{13} \mathrm{C}$ NMR spectra of $\mathbf{3} \mathbf{x}\left(\mathrm{CDCl}_{3}, 100 \mathrm{M}\right)$. 


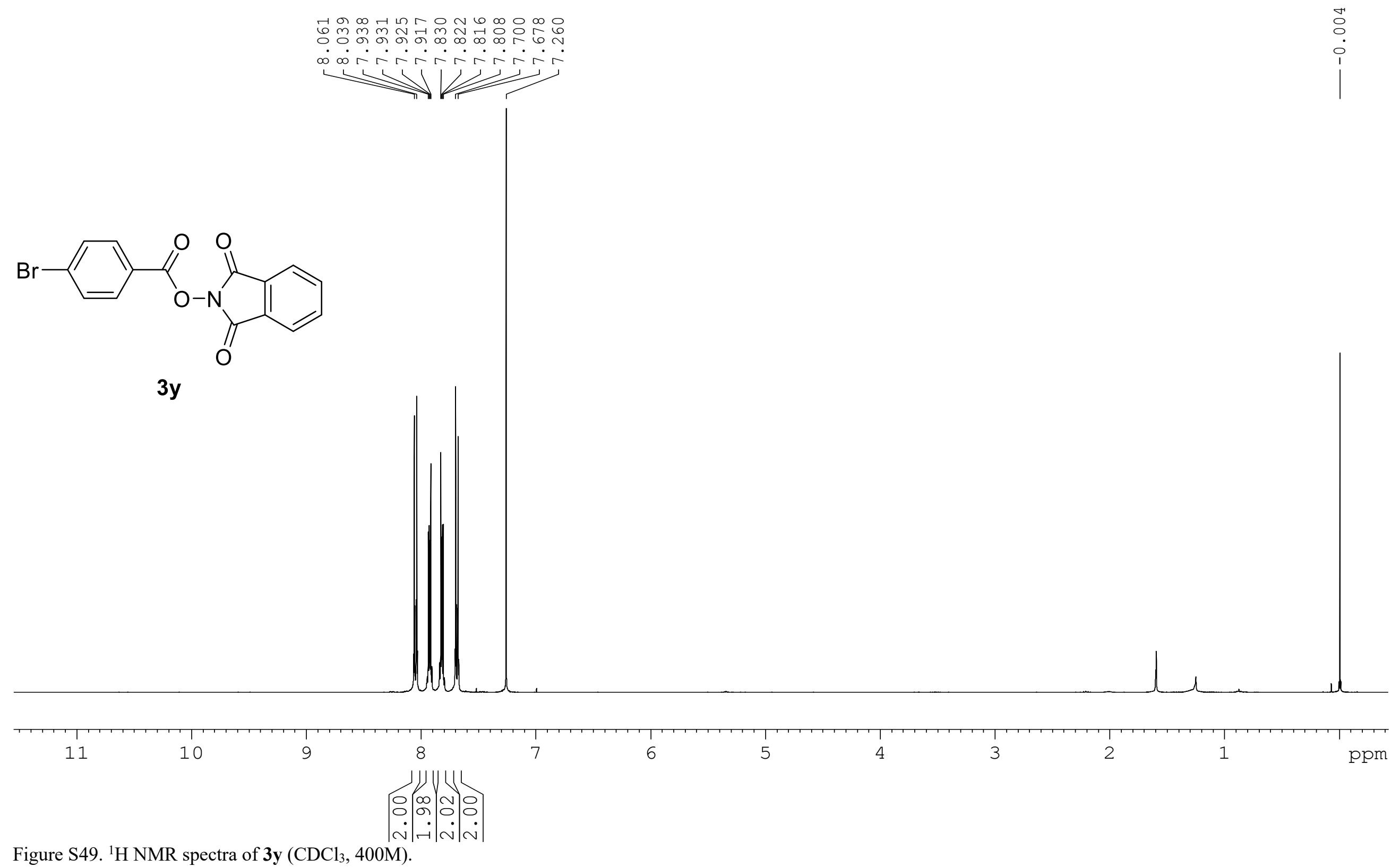



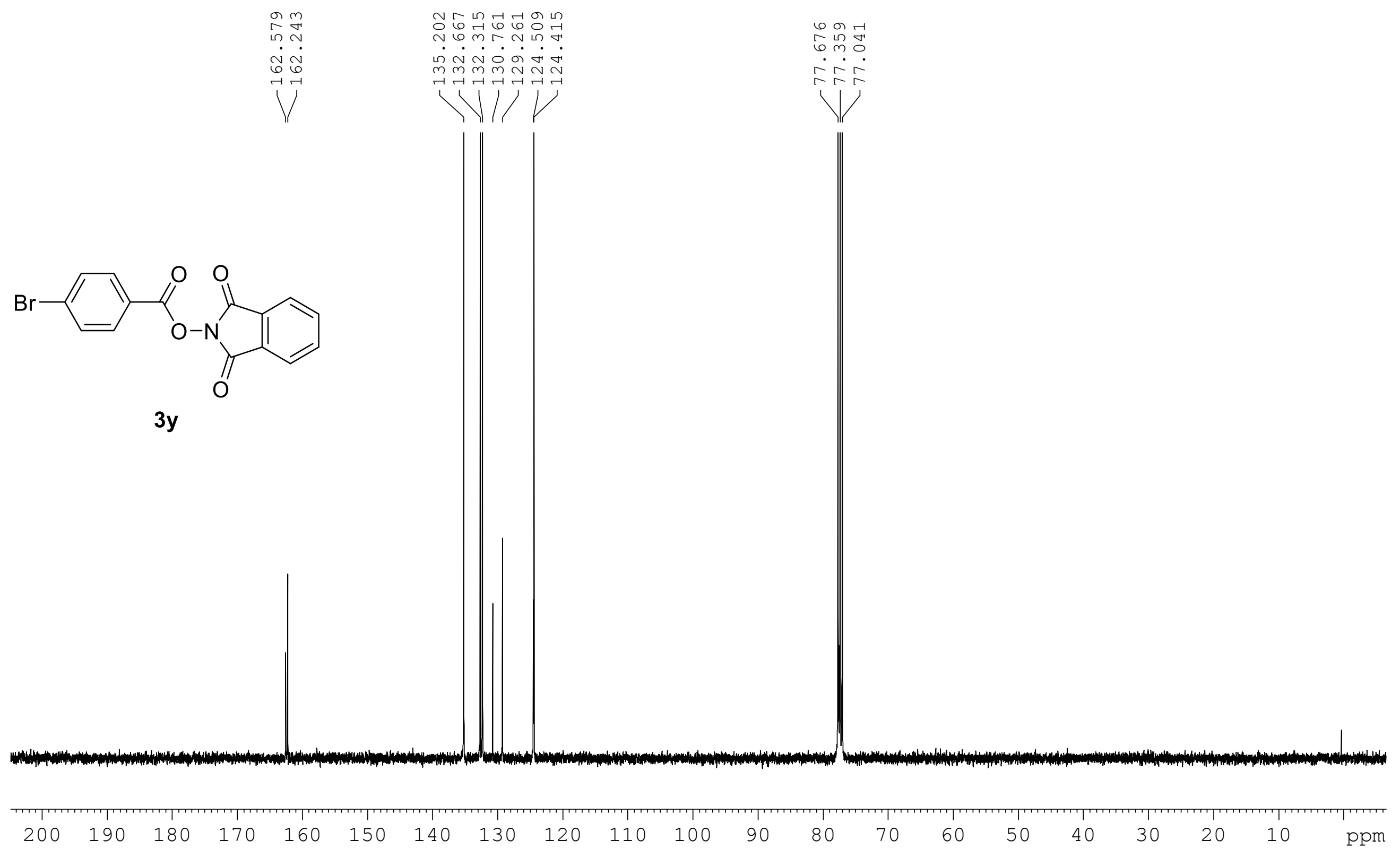

20

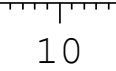

ppm

Figure S50. ${ }^{13} \mathrm{C}$ NMR spectra of $\mathbf{3 y}\left(\mathrm{CDCl}_{3}, 100 \mathrm{M}\right)$. 


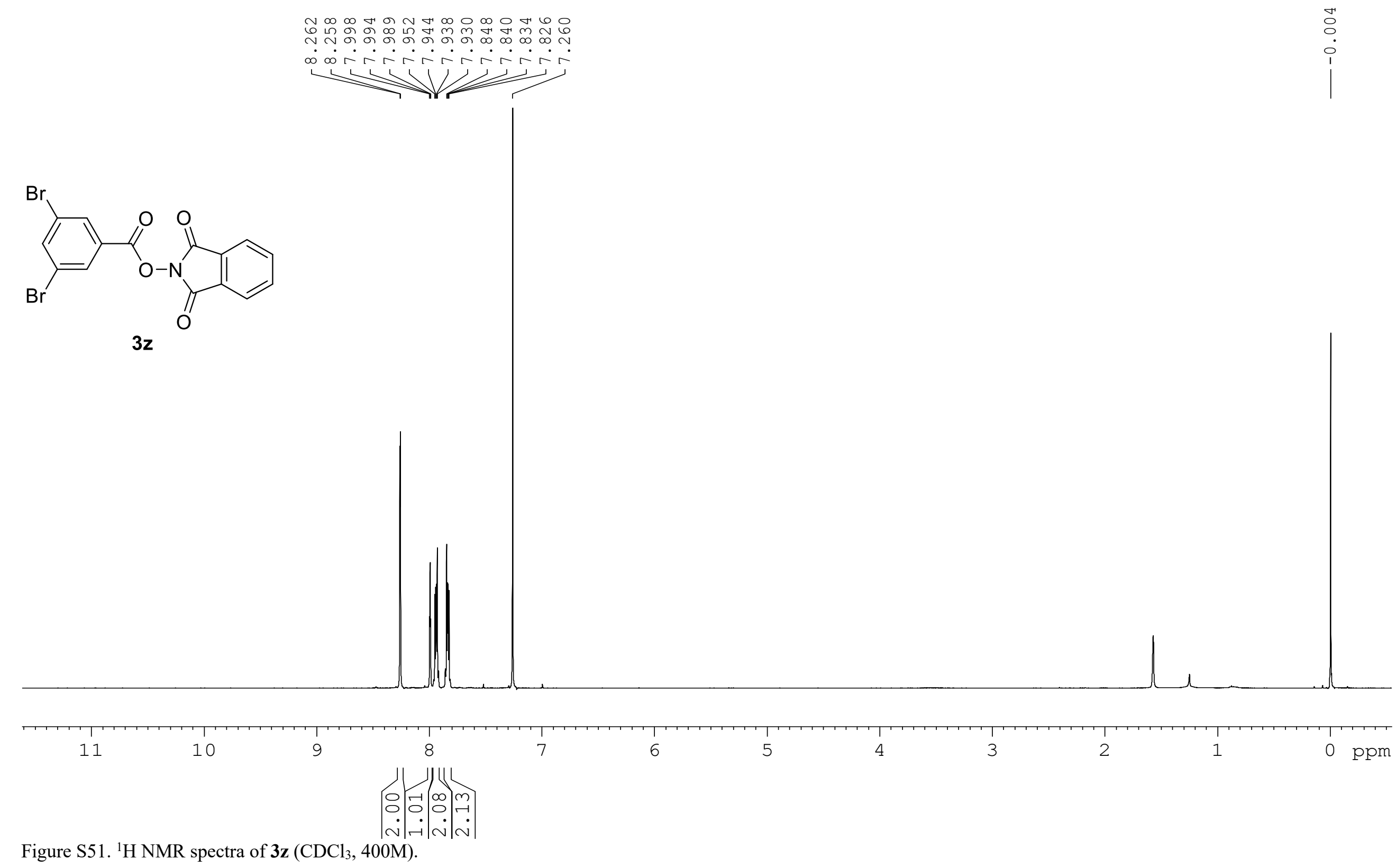




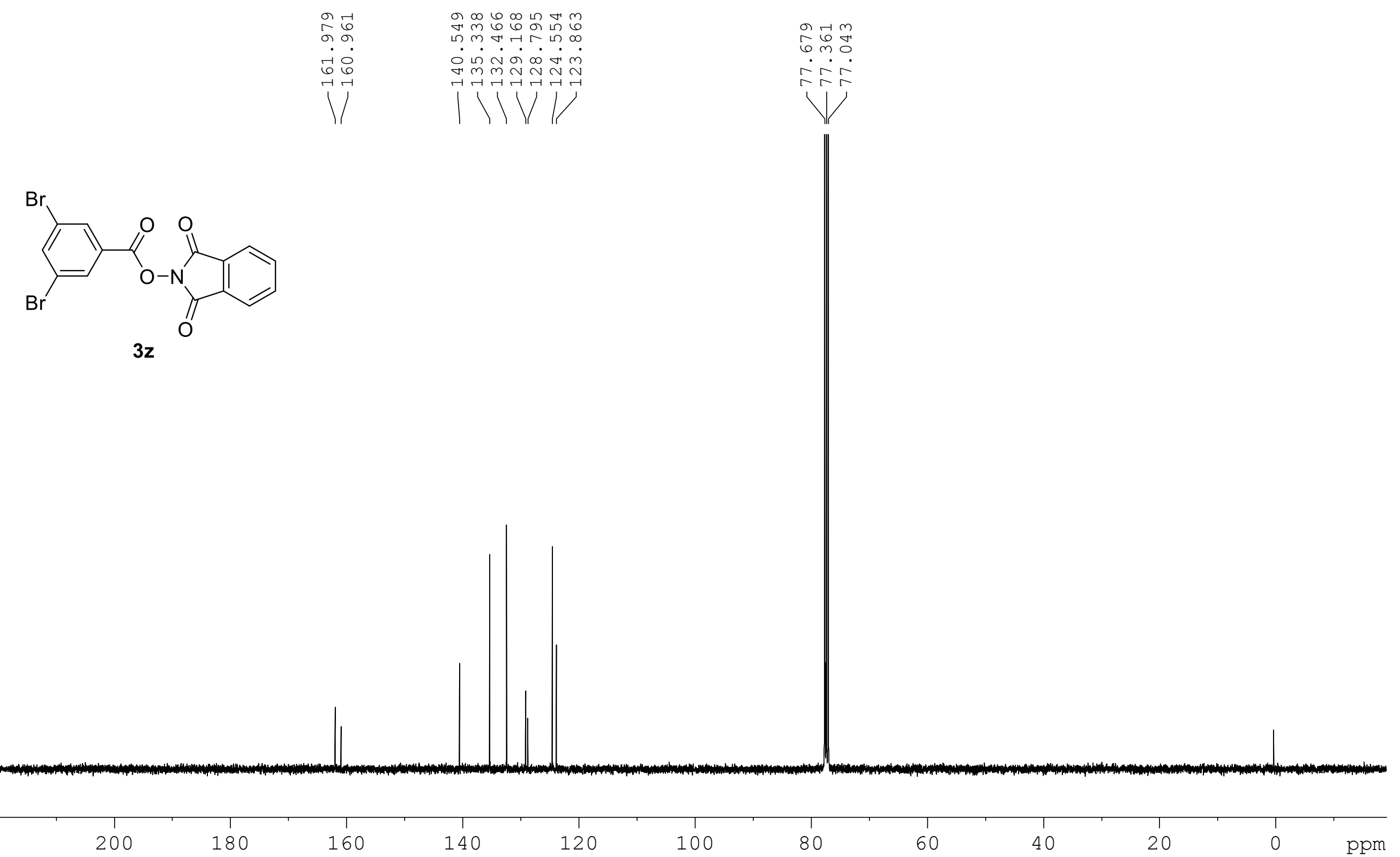

Figure $\mathrm{S} 52 .{ }^{13} \mathrm{C}$ NMR spectra of $\mathbf{3 z}\left(\mathrm{CDCl}_{3}, 100 \mathrm{M}\right)$. 
<smiles>O=C(ON1C(=O)c2ccccc2C1=O)c1ccc(I)cc1</smiles>

$\overrightarrow{0}$
0
0
1

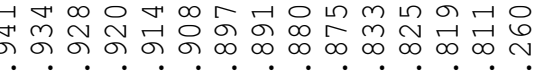

BA
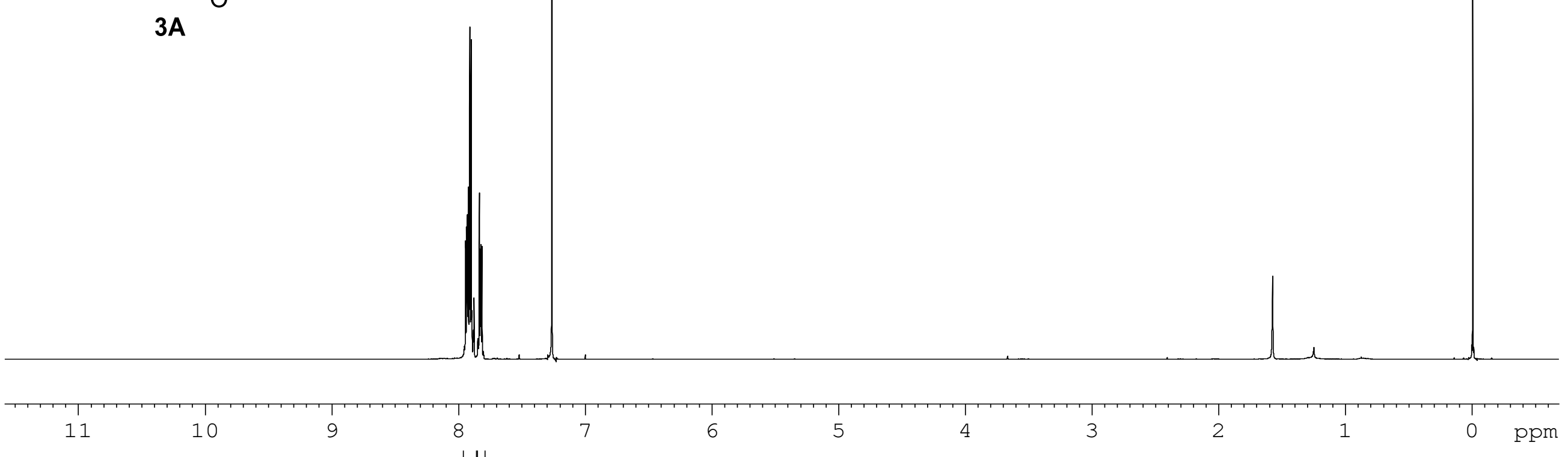

$\left(\begin{array}{c|c}\infty & \\ 0 & 8 \\ 0 & 0 \\ \dot{0} & \dot{v}\end{array} \mid\right.$

Figure S53. ${ }^{1} \mathrm{H}$ NMR spectra of $\mathbf{3 A}\left(\mathrm{CDCl}_{3}, 400 \mathrm{M}\right)$.

S-71 

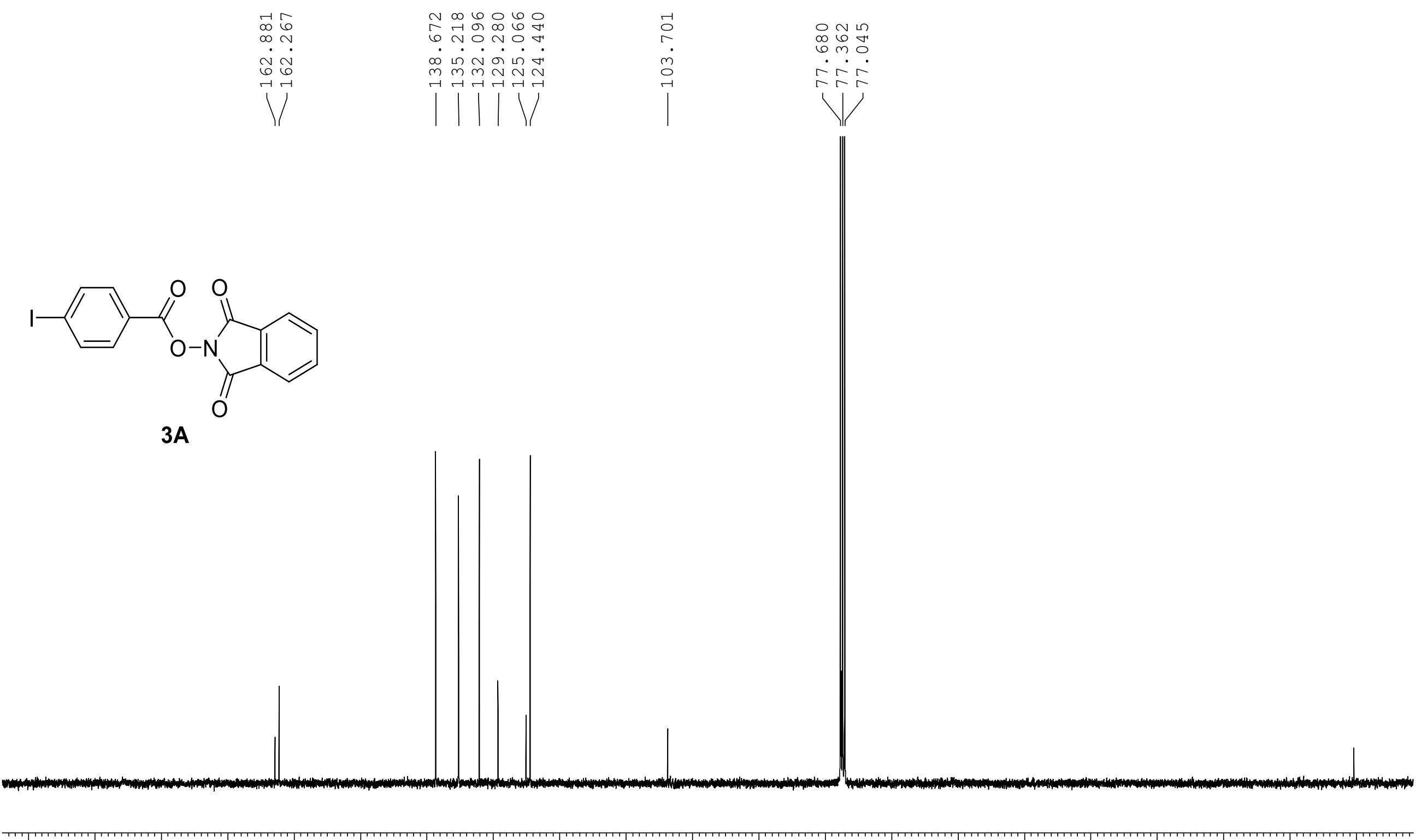

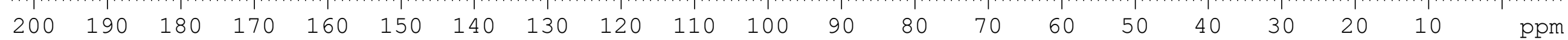
Figure S54. ${ }^{13} \mathrm{C}$ NMR spectra of $\mathbf{3 A}\left(\mathrm{CDCl}_{3}, 100 \mathrm{M}\right)$. 


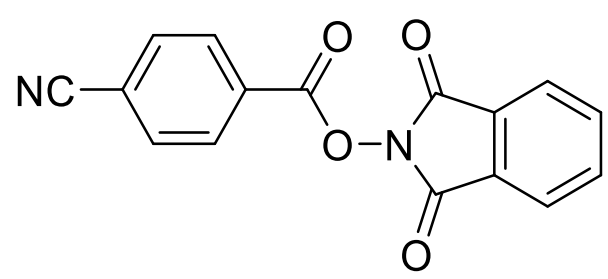

3B

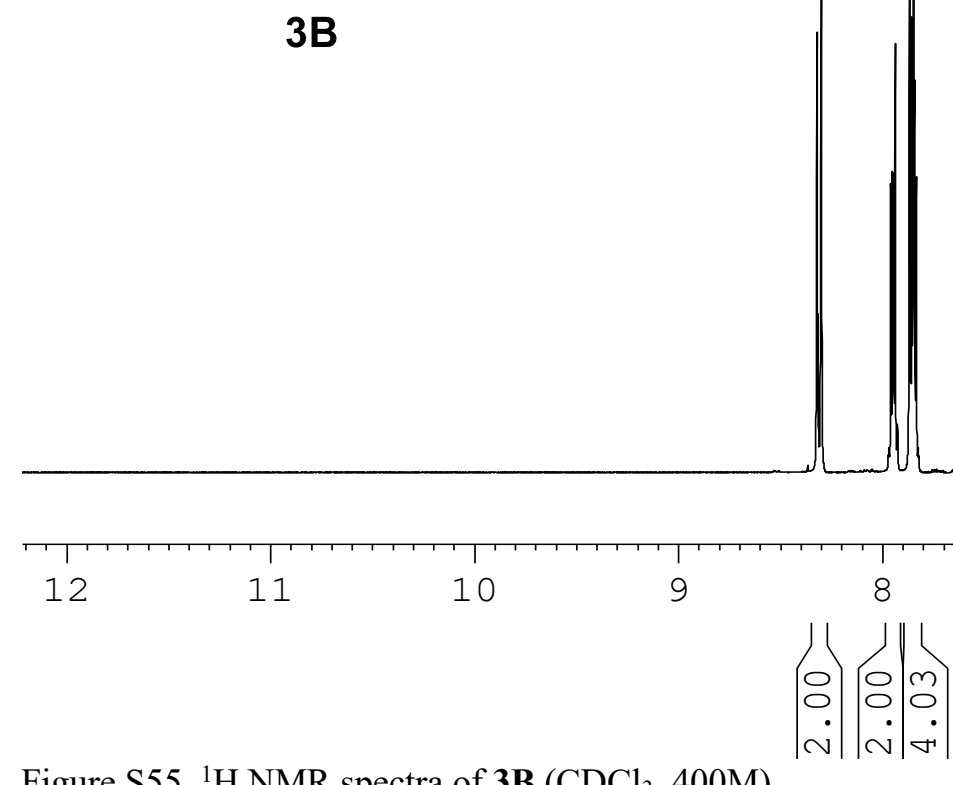

Figure S55. ${ }^{1} \mathrm{H}$ NMR spectra of $\mathbf{3 B}\left(\mathrm{CDCl}_{3}, 400 \mathrm{M}\right)$. 

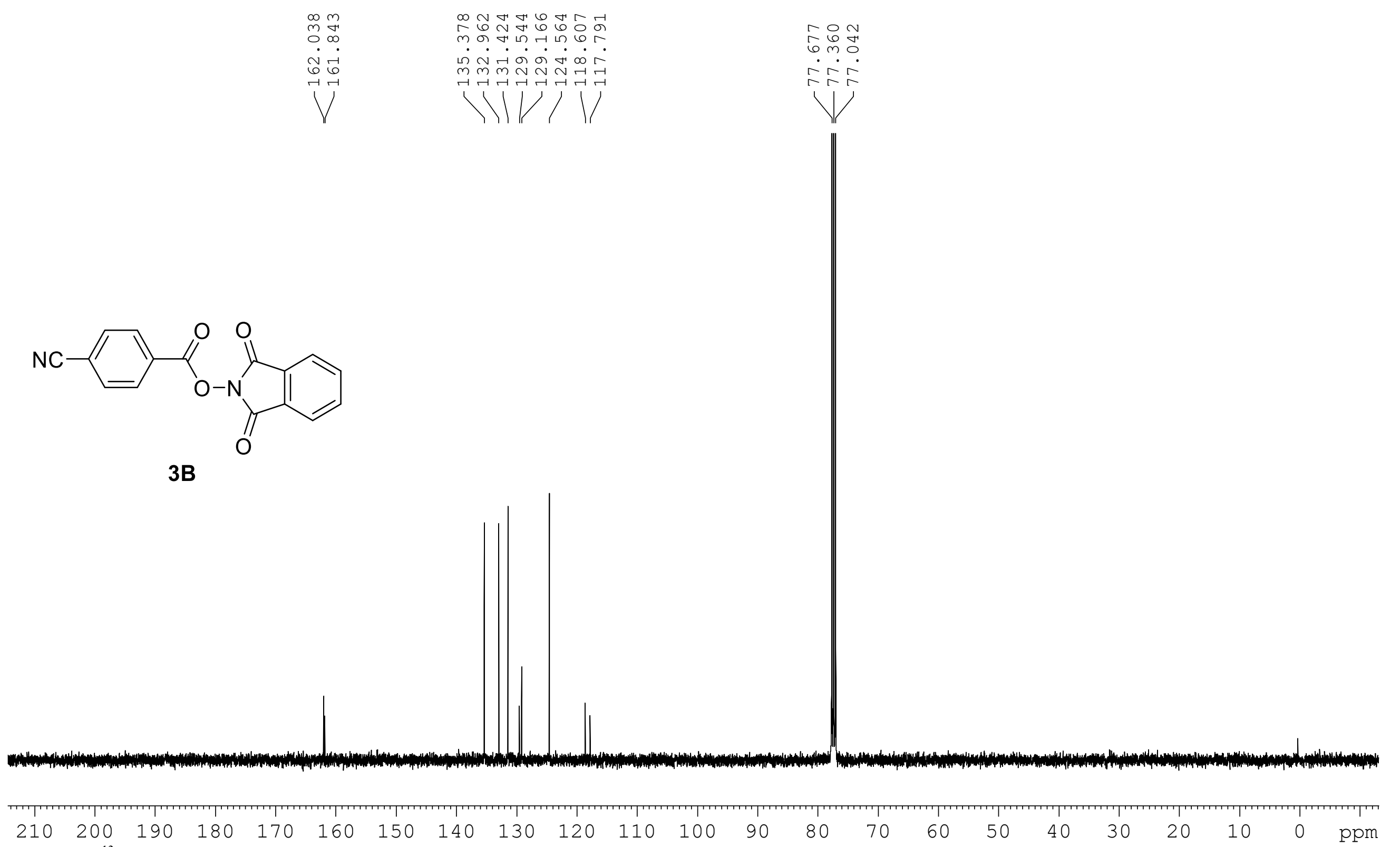

Figure S56. ${ }^{13} \mathrm{C}$ NMR spectra of $3 B\left(\mathrm{CDCl}_{3}, 100 \mathrm{M}\right)$. 
<smiles>COC(=O)c1ccc(C(=O)ON2C(=O)c3ccccc3C2=O)cc1</smiles>

$3 C$

$\overrightarrow{1}$
0
0

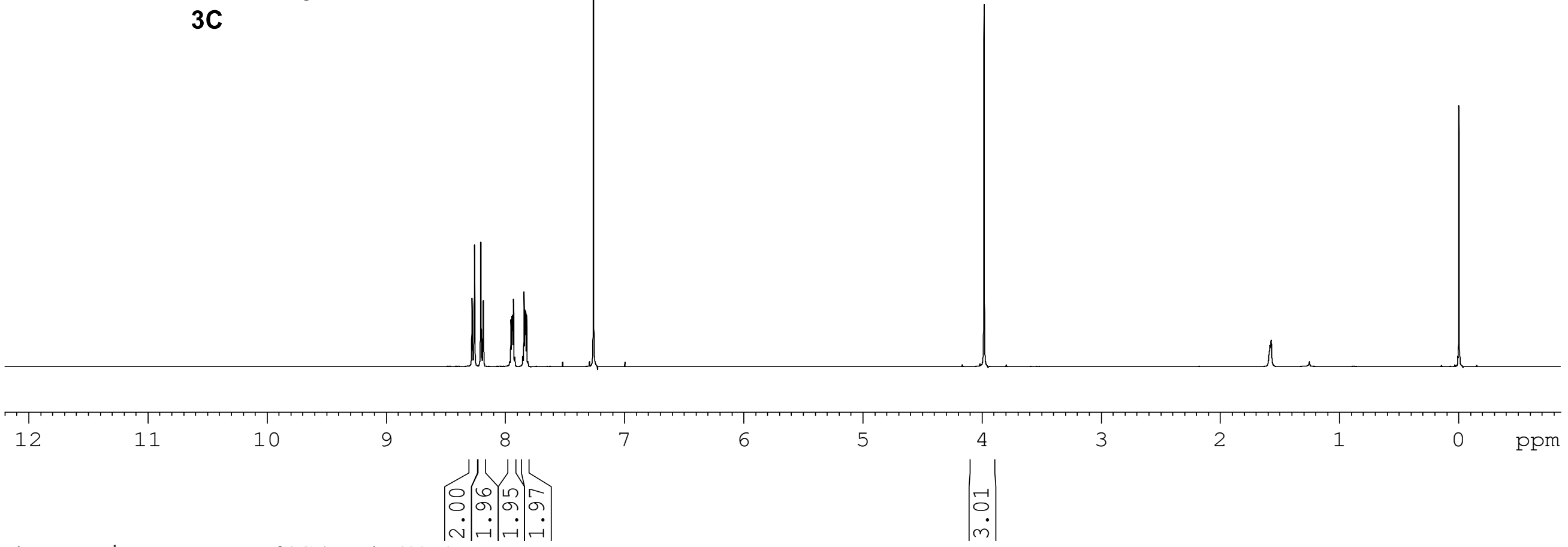

Figure S57. ${ }^{1} \mathrm{H}$ NMR spectra of $\mathbf{3 C}\left(\mathrm{CDCl}_{3}, 400 \mathrm{M}\right)$.

S-75 


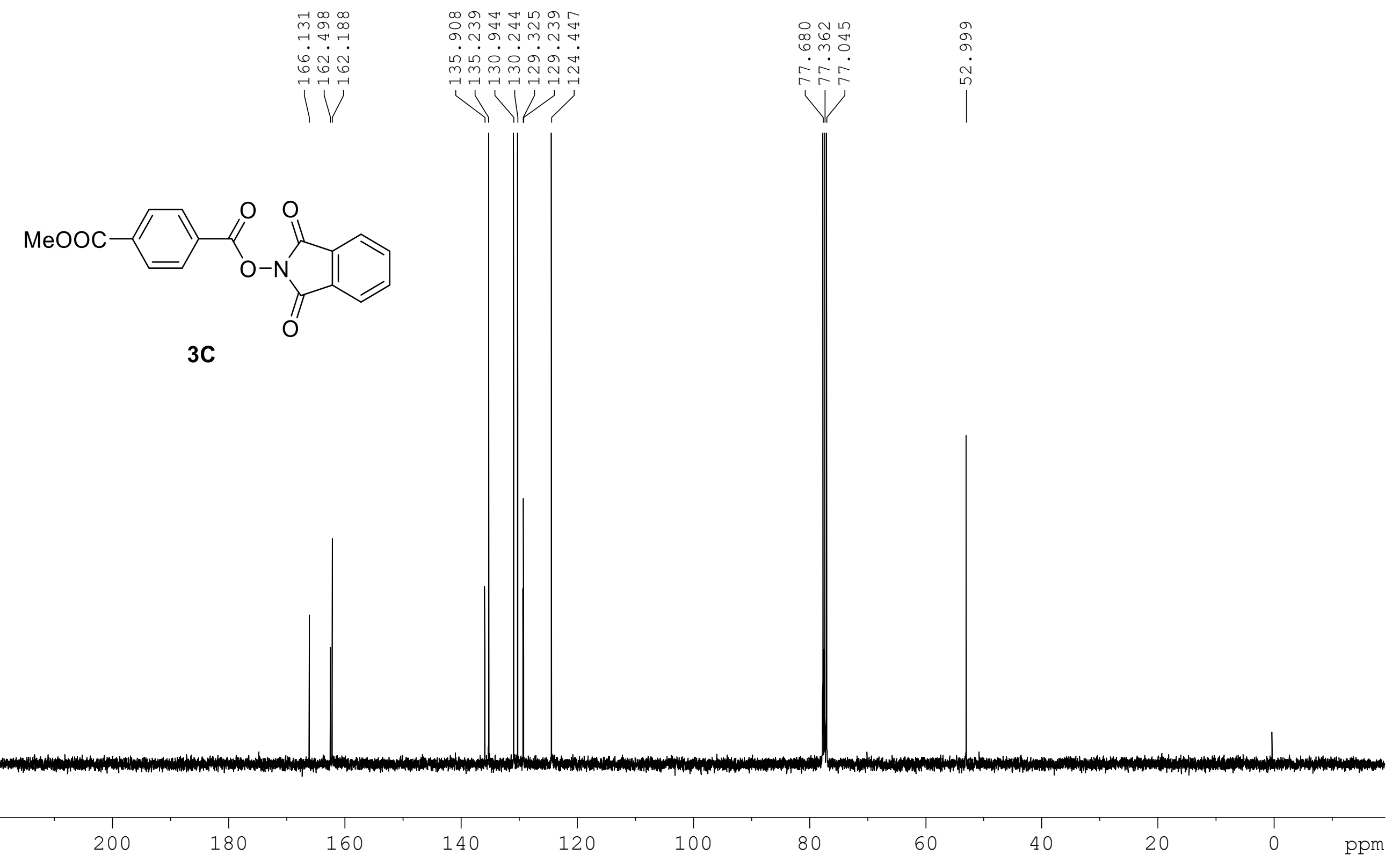

Figure $\mathrm{S} 58 .{ }^{13} \mathrm{C}$ NMR spectra of $\mathbf{3 C}\left(\mathrm{CDCl}_{3}, 100 \mathrm{M}\right)$. 


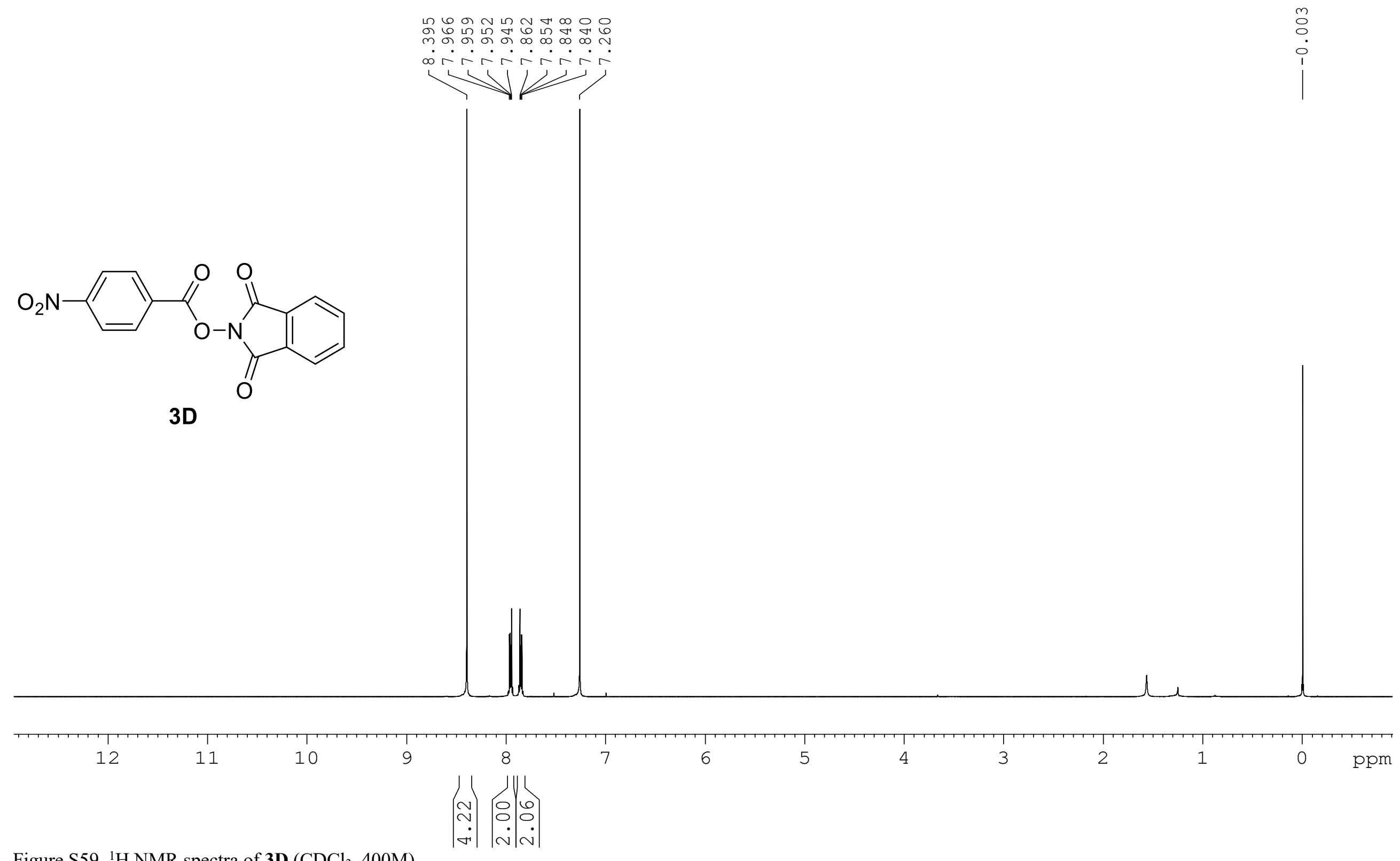

Figure S59. ${ }^{1} \mathrm{H}$ NMR spectra of $3 D\left(\mathrm{CDCl}_{3}, 400 \mathrm{M}\right)$. 

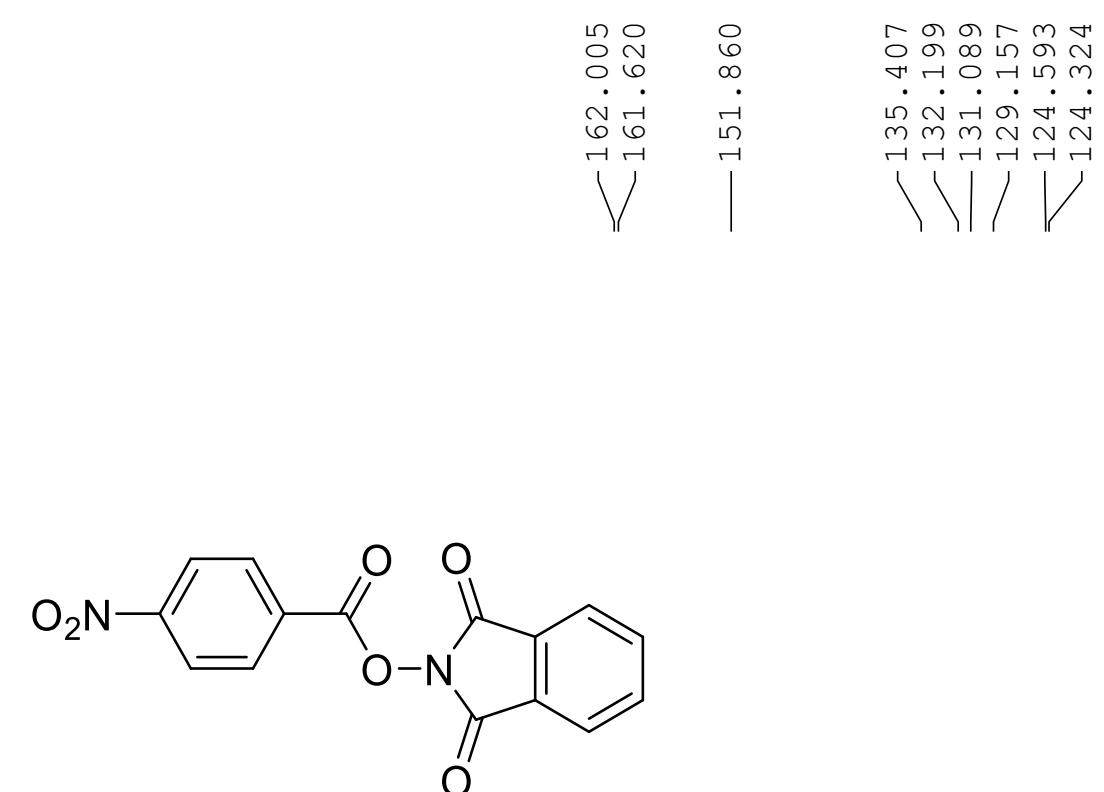

3D

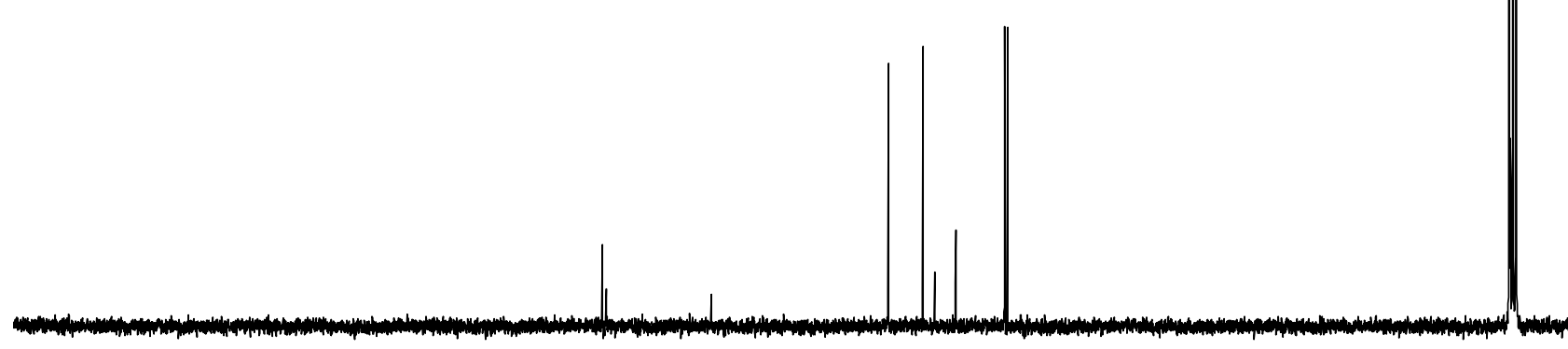




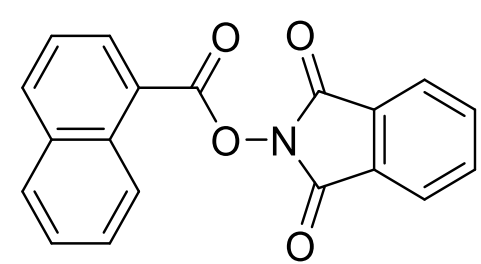

$3 E$

\section{undilit}

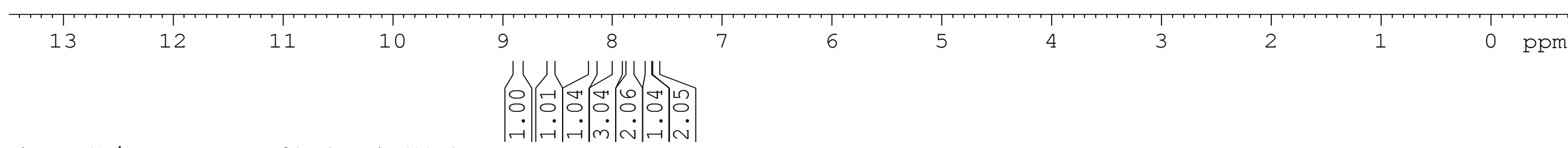

Figure S61. ${ }^{1} \mathrm{H}$ NMR spectra of $\mathbf{3 E}\left(\mathrm{CDCl}_{3}, 400 \mathrm{M}\right)$. 


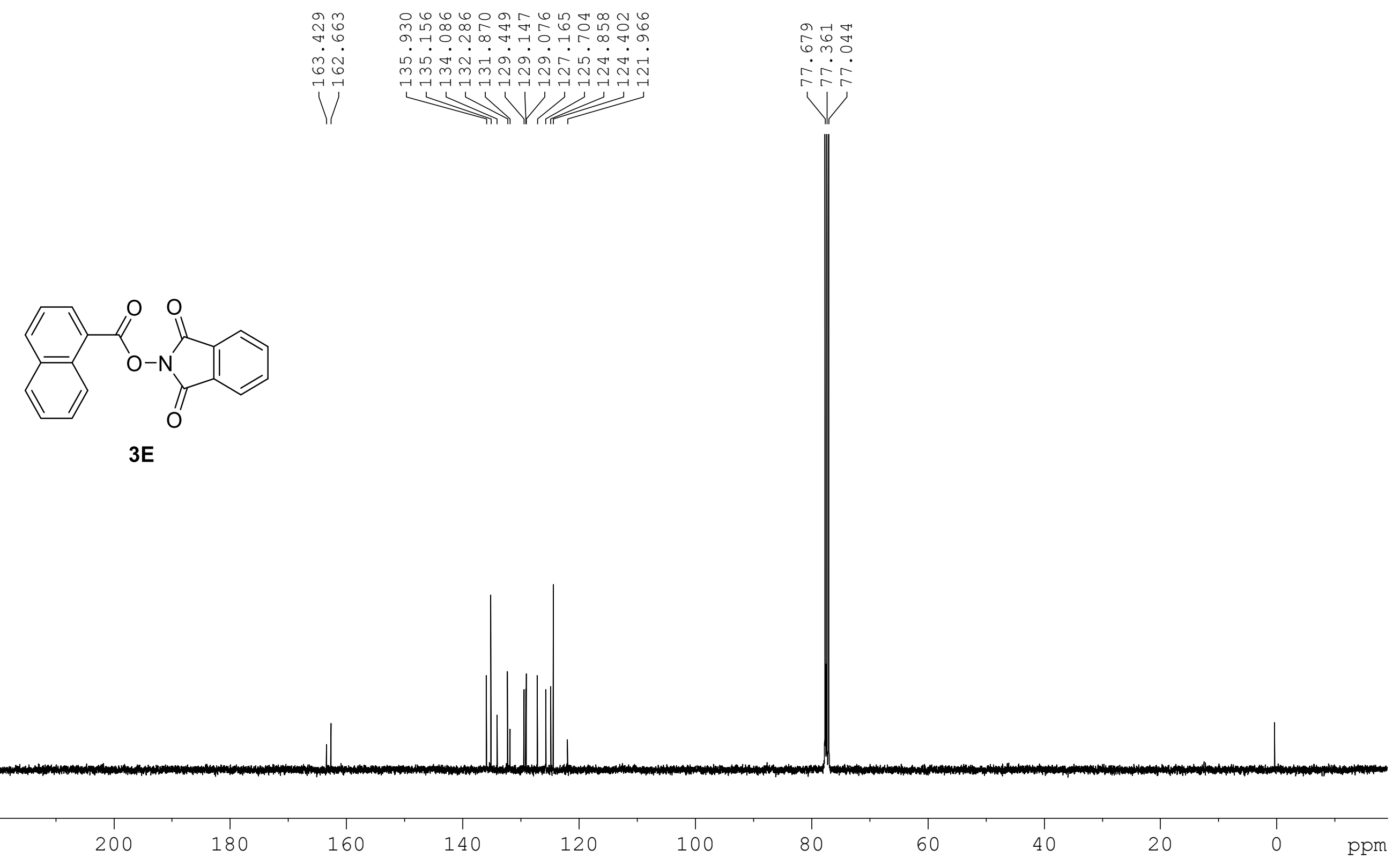

Figure $\mathrm{S} 62 .{ }^{13} \mathrm{C}$ NMR spectra of $\mathbf{3 E}\left(\mathrm{CDCl}_{3}, 100 \mathrm{M}\right)$. 


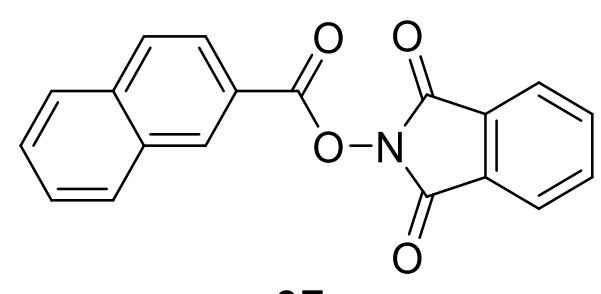

3F
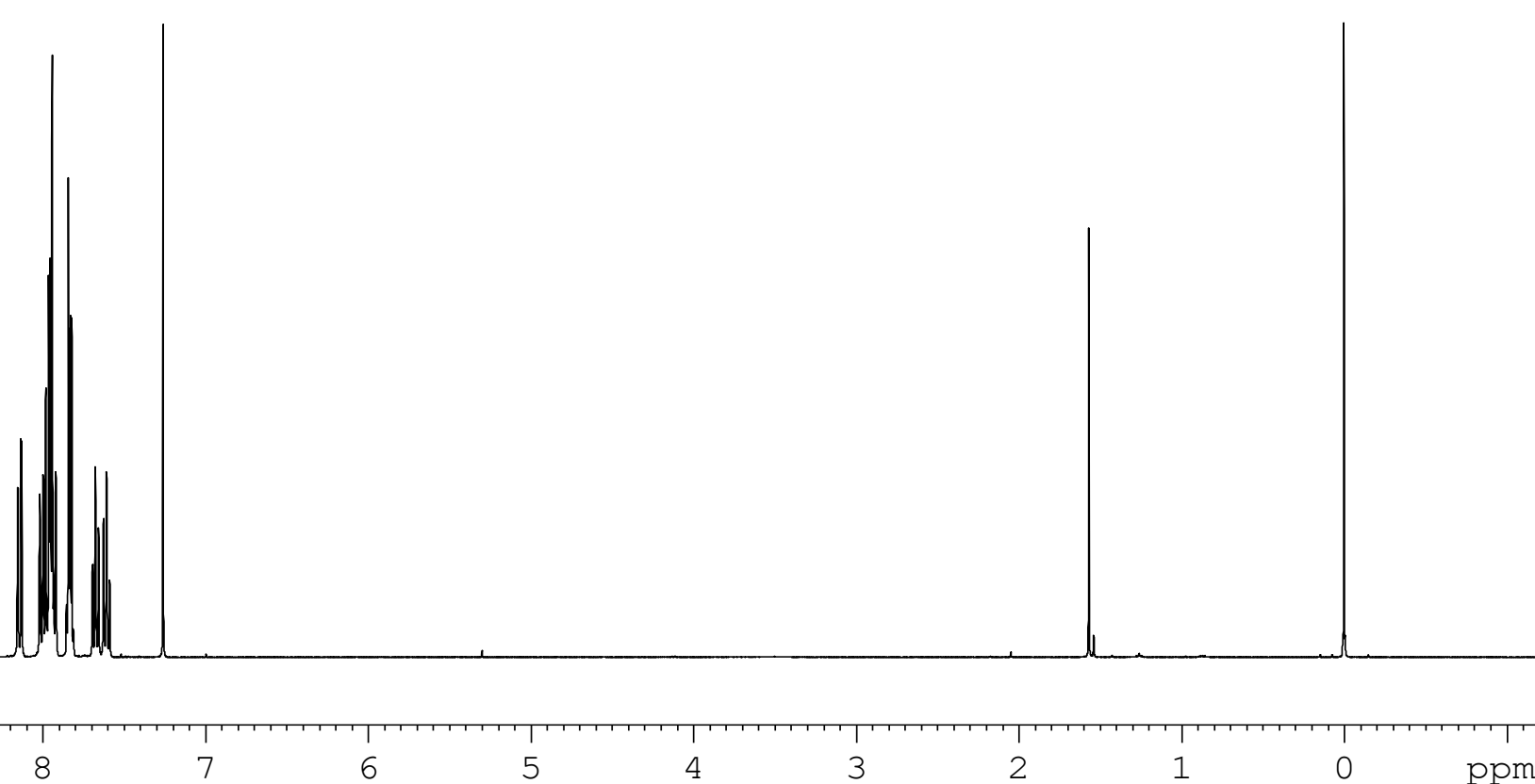

13

12

11

10

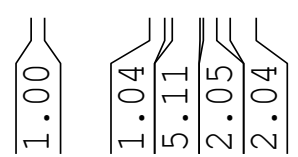

Figure S63. ${ }^{1} \mathrm{H}$ NMR spectra of $\mathbf{3 F}\left(\mathrm{CDCl}_{3}, 400 \mathrm{M}\right)$ 


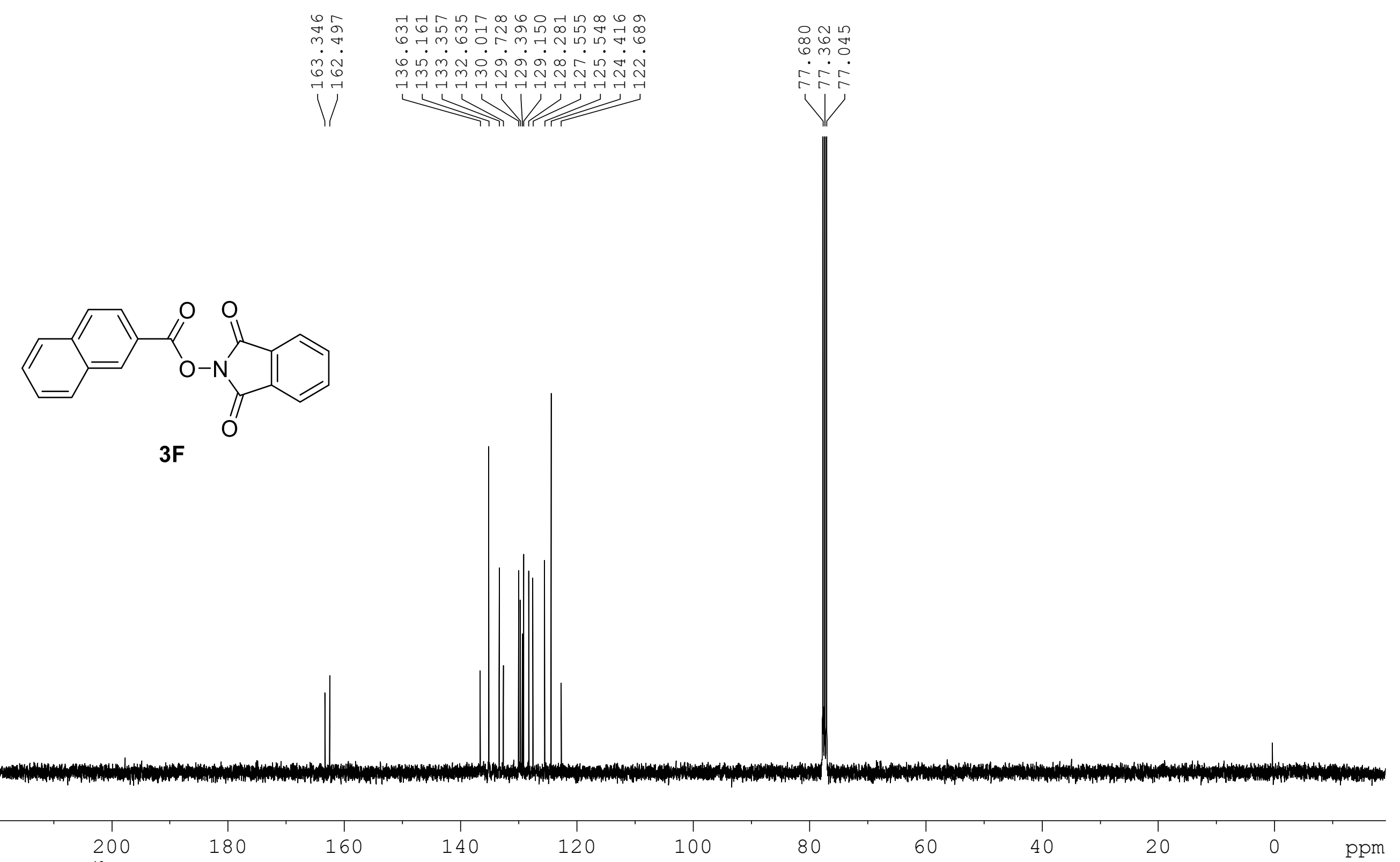

Figure S64. ${ }^{13} \mathrm{C}$ NMR spectra of $\mathbf{3 F}\left(\mathrm{CDCl}_{3}, 100 \mathrm{M}\right)$. 
<smiles>O=C(ON1C(=O)c2ccccc2C1=O)c1cccs1</smiles>

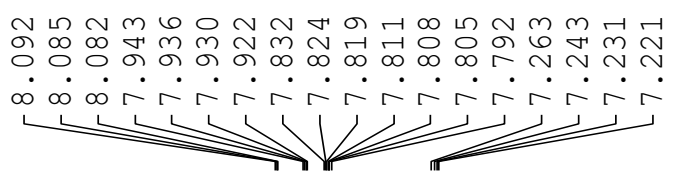

8
0
0
1
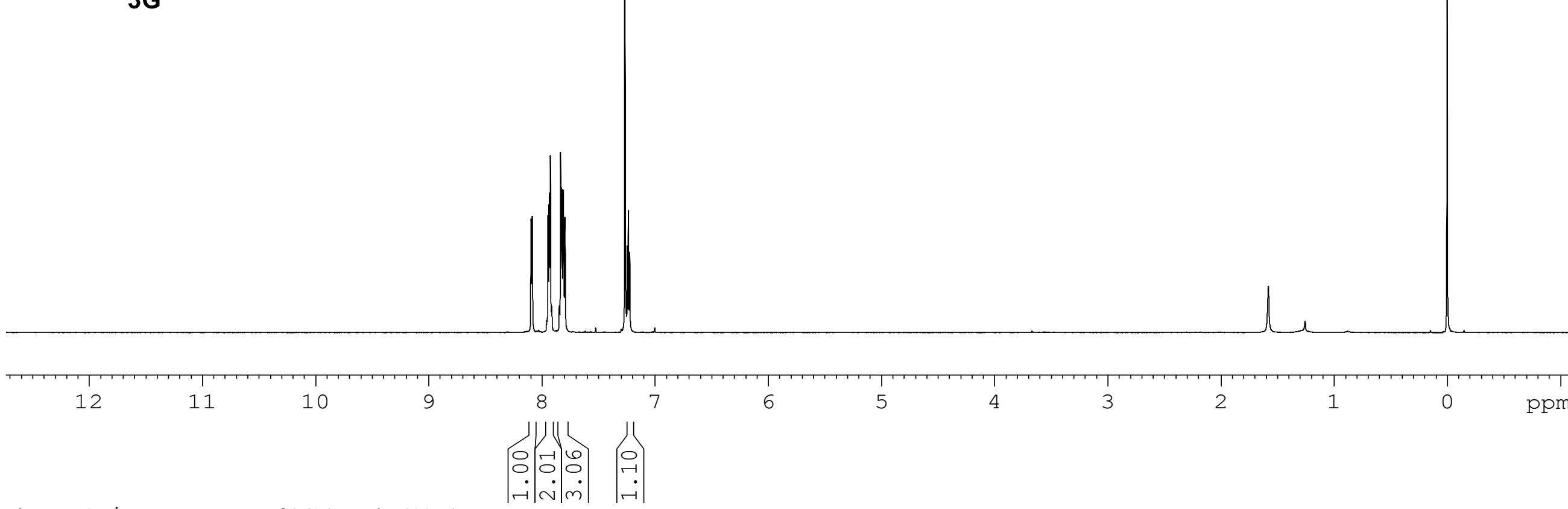

Figure S65. ${ }^{1} \mathrm{H}$ NMR spectra of $\mathbf{3 G}\left(\mathrm{CDCl}_{3}, 400 \mathrm{M}\right)$.

S-83 


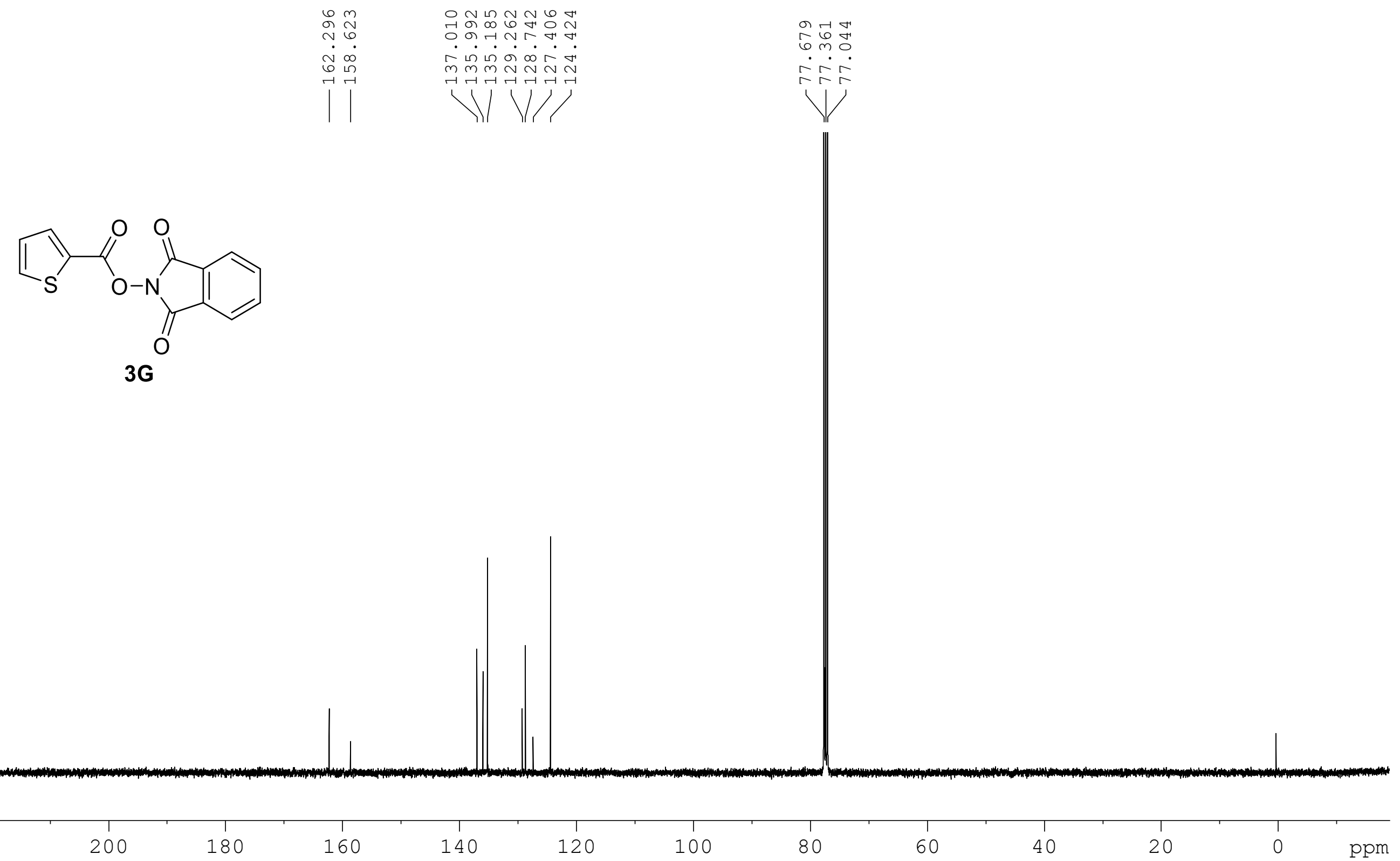

Figure $\mathrm{S} 66 .{ }^{13} \mathrm{C}$ NMR spectra of $\mathbf{3 G}\left(\mathrm{CDCl}_{3}, 100 \mathrm{M}\right)$. 


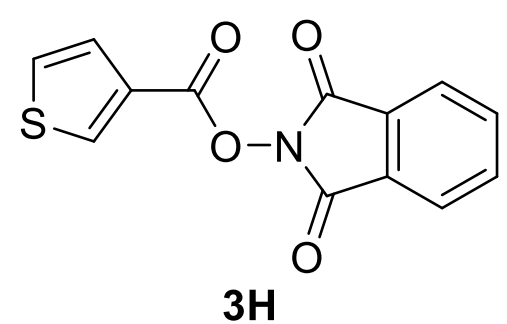

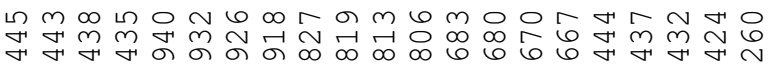

$\infty \infty_{\infty} \infty \dot{0}+\dot{0}$
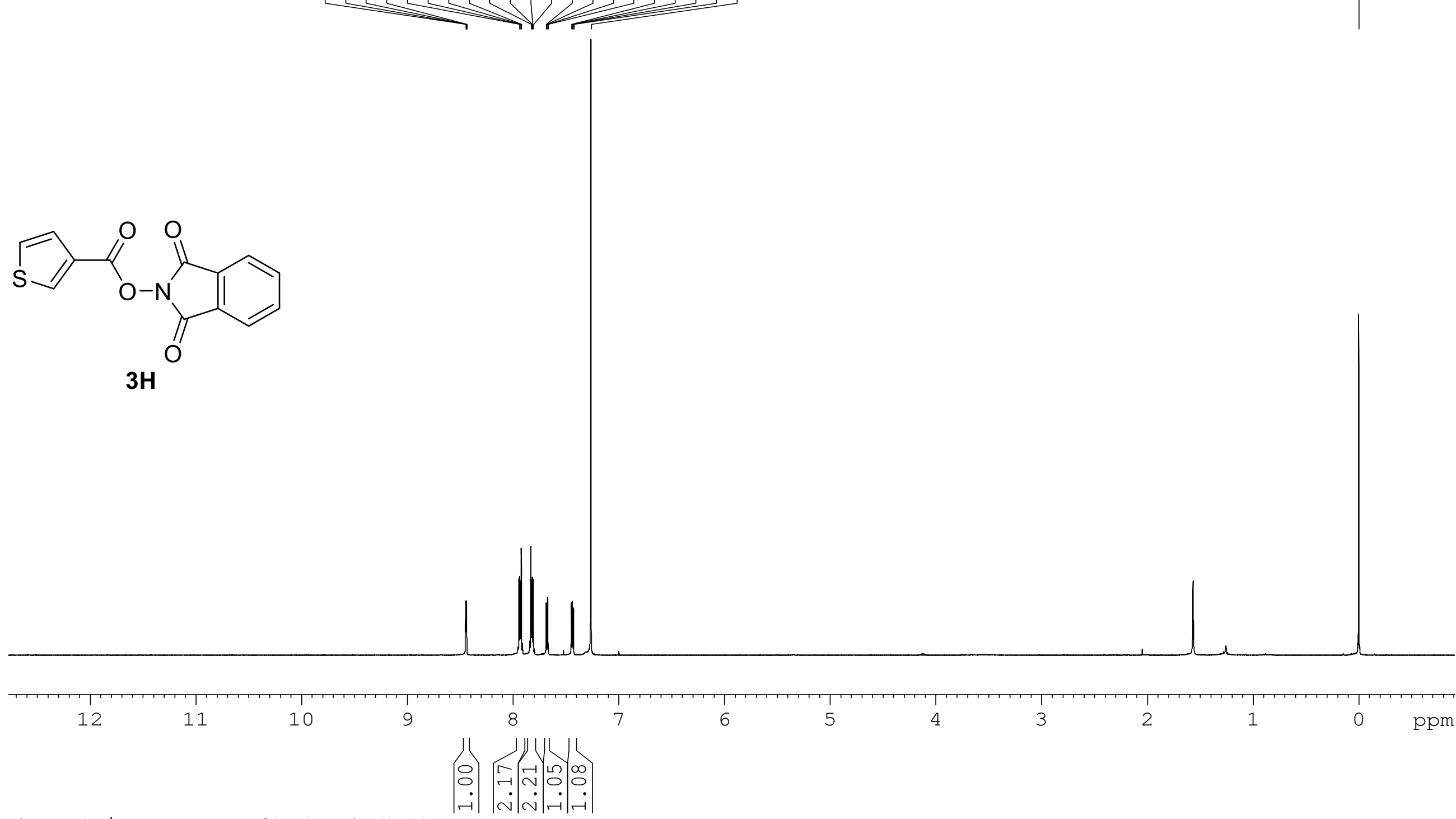

Figure S67. ${ }^{1} \mathrm{H}$ NMR spectra of $\mathbf{3 H}\left(\mathrm{CDCl}_{3}, 400 \mathrm{M}\right)$ 


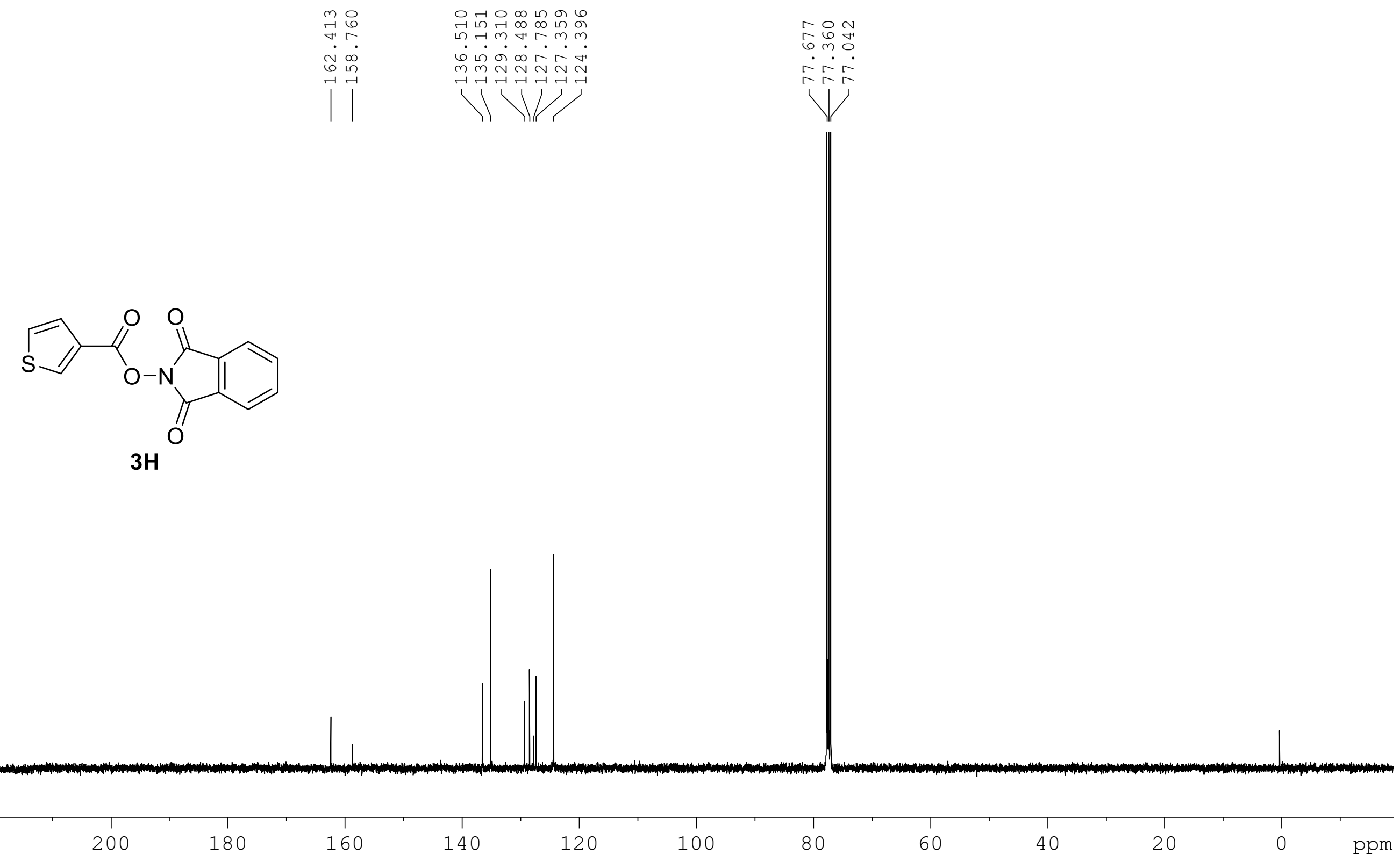

Figure $\mathrm{S} 68 .{ }^{13} \mathrm{C}$ NMR spectra of $\mathbf{3 H}\left(\mathrm{CDCl}_{3}, 100 \mathrm{M}\right)$. 

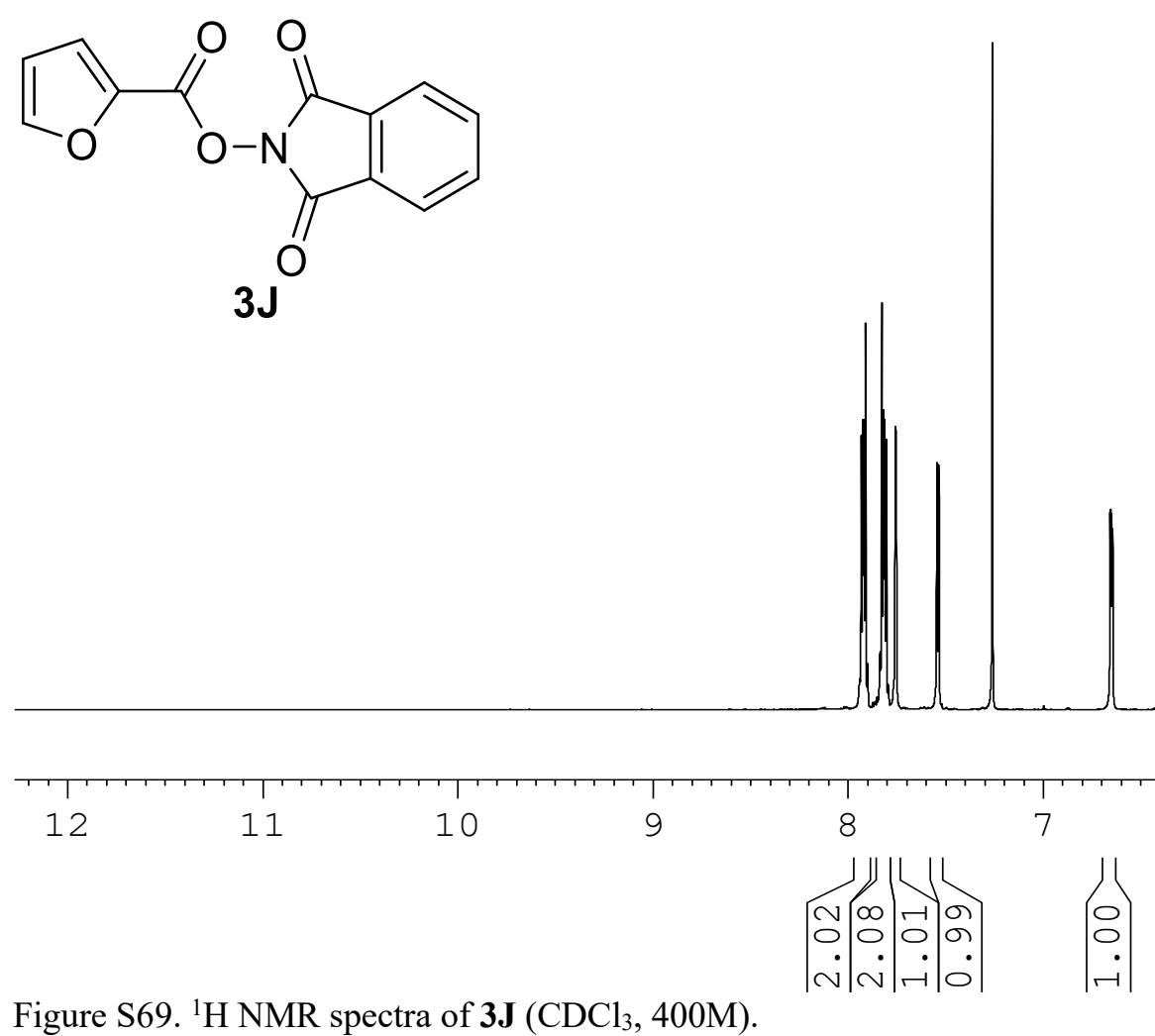

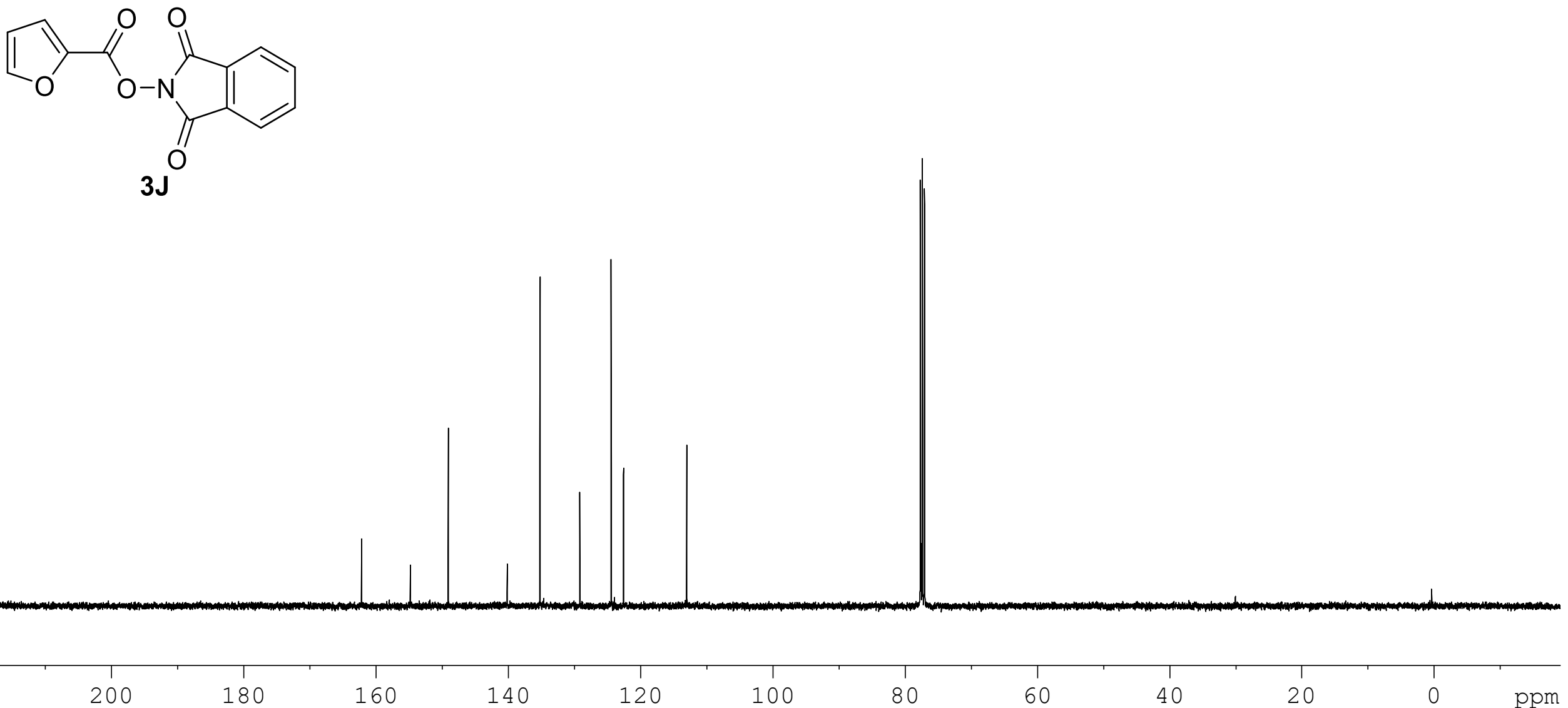

Figure S70. ${ }^{13} \mathrm{C}$ NMR spectra of $\mathbf{3 J}\left(\mathrm{CDCl}_{3}, 100 \mathrm{M}\right)$. 


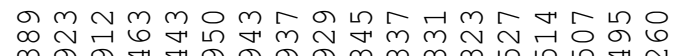

$i_{\infty}^{\infty} \sin ^{2}$
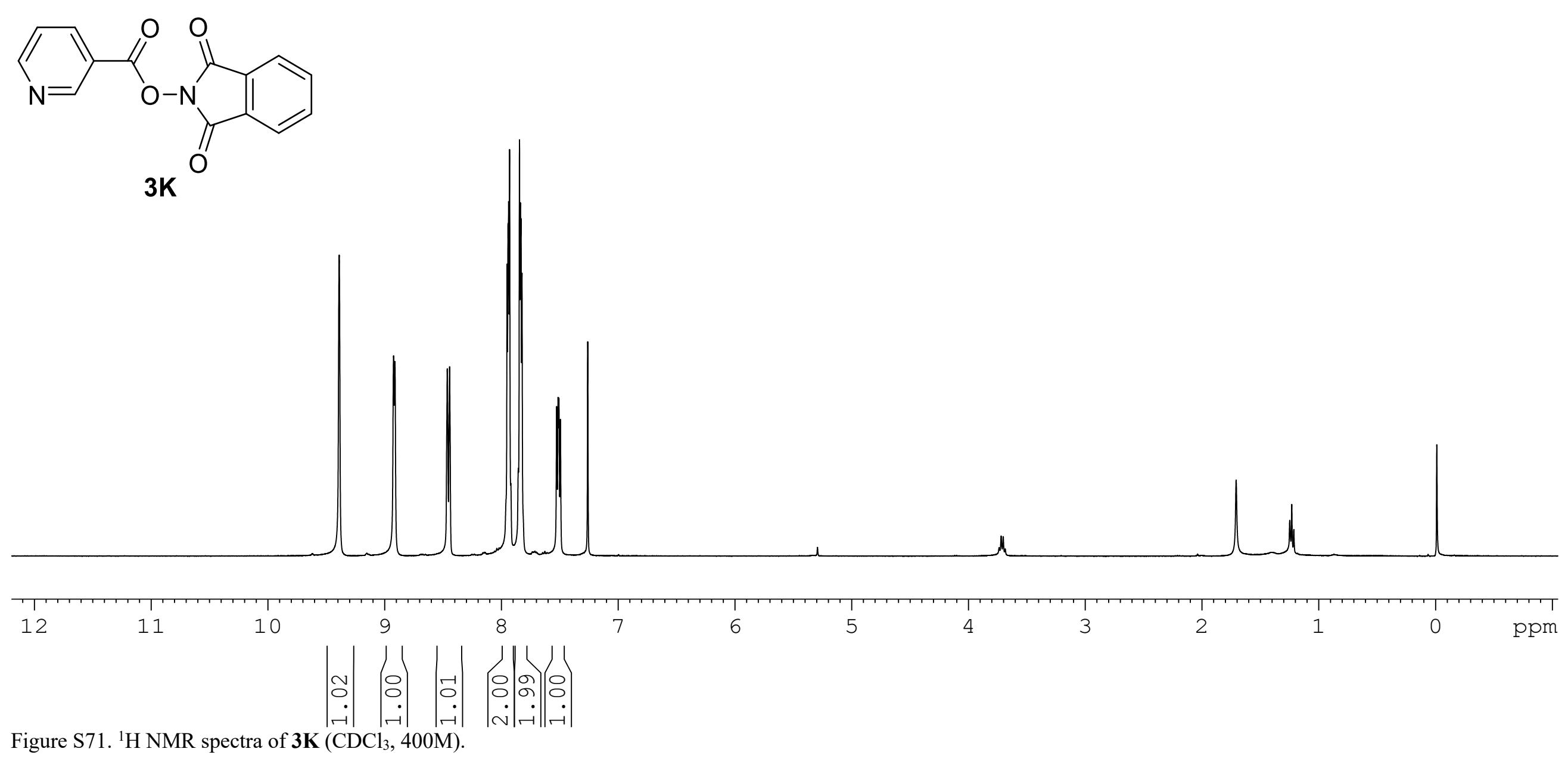

Figure S71. ${ }^{1} \mathrm{H}$ NMR spectra of $\mathbf{3 K}\left(\mathrm{CDCl}_{3}, 400 \mathrm{M}\right)$. 


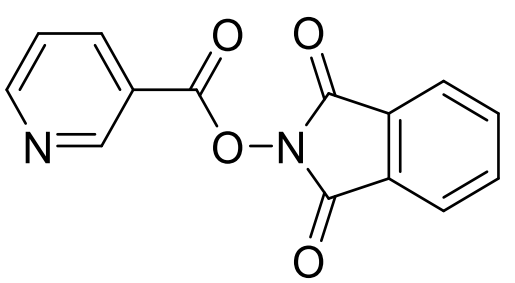

3K
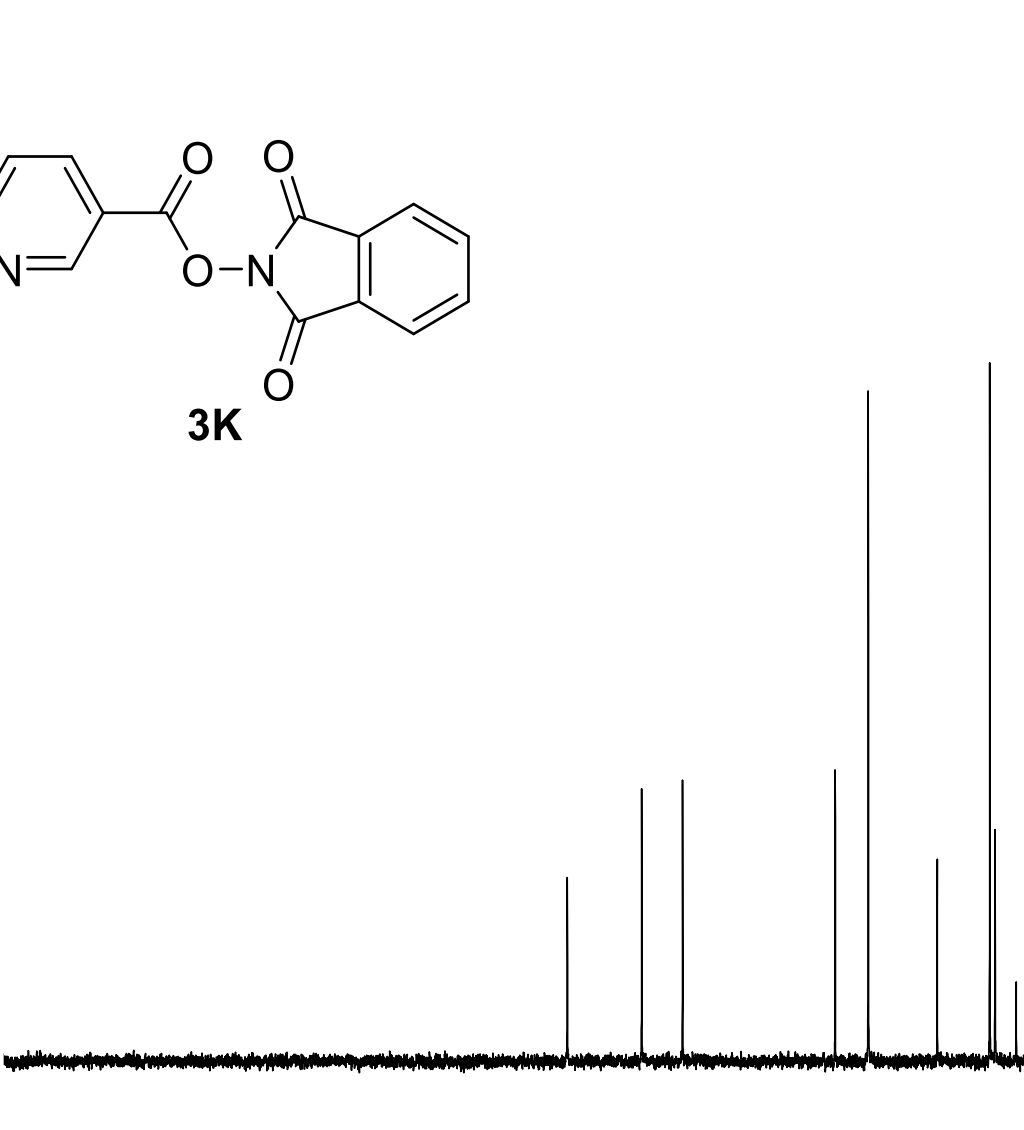

$$
200 \quad 190 \quad 180 \quad 170 \quad 160 \quad 150
$$

Figure $\mathrm{S} 72 .{ }^{13} \mathrm{C}$ NMR spectra of $\mathbf{3 K}\left(\mathrm{CDCl}_{3}, 100 \mathrm{M}\right)$ 


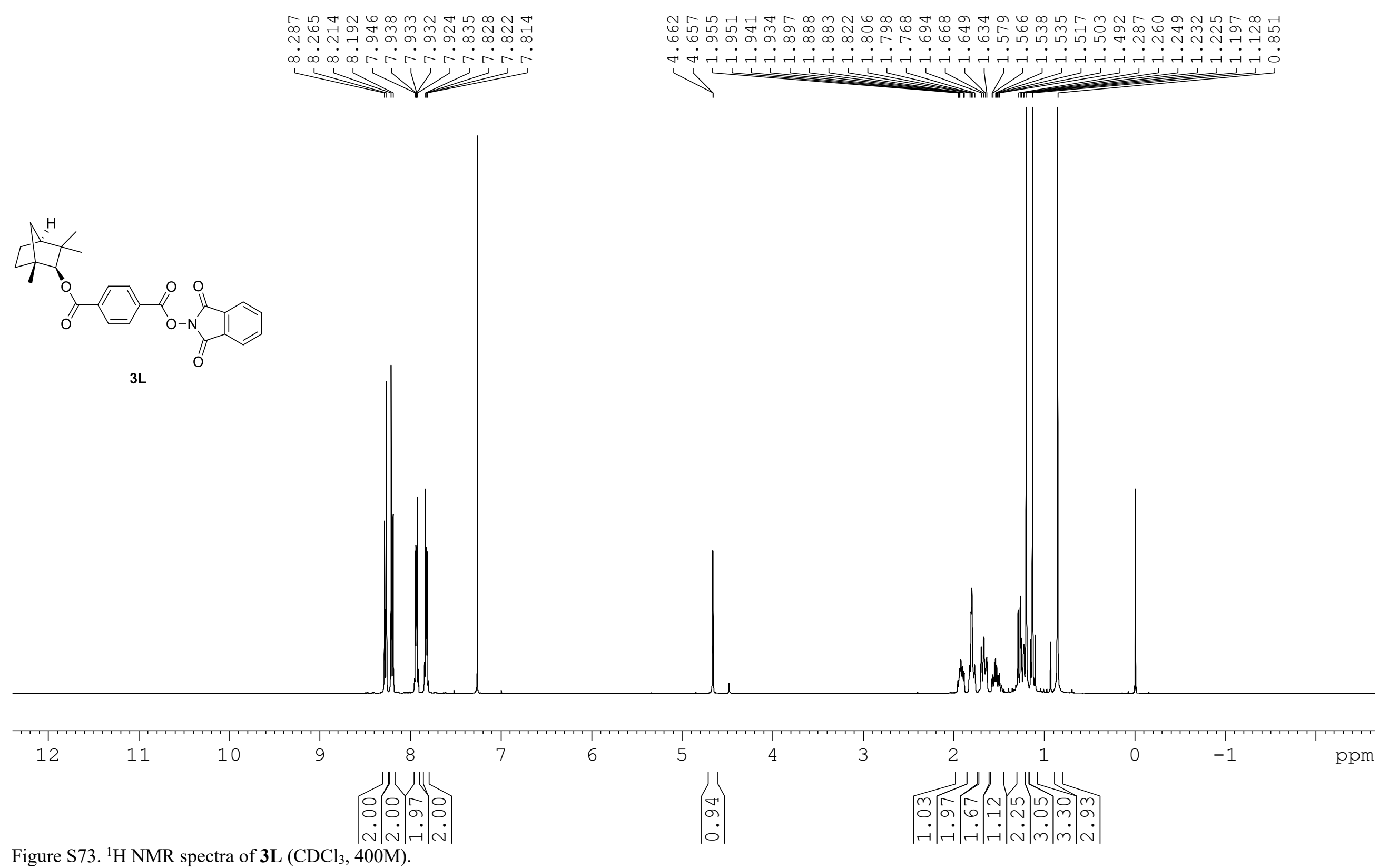




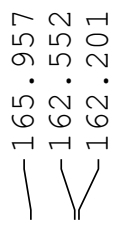

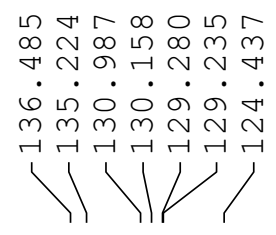

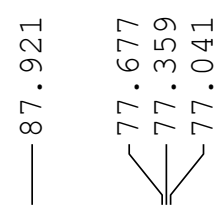

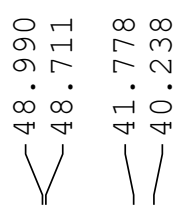
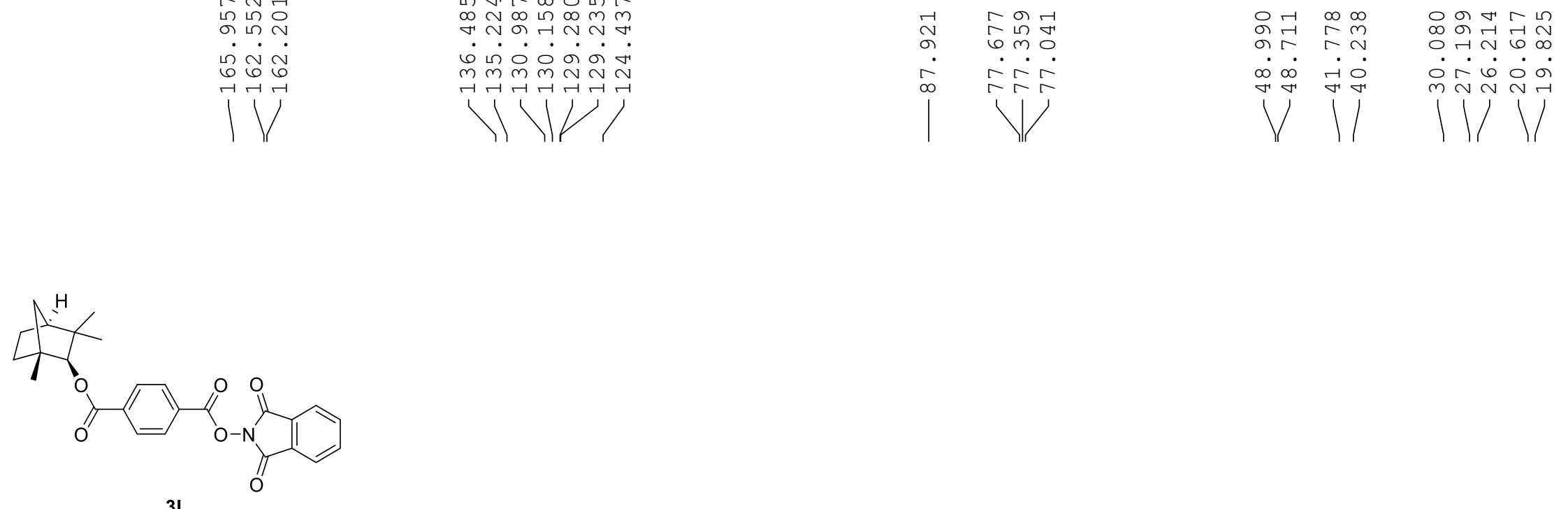

BL

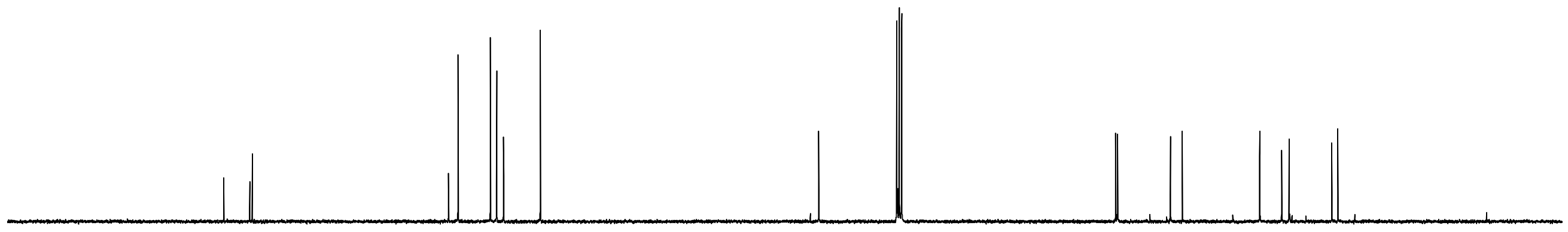

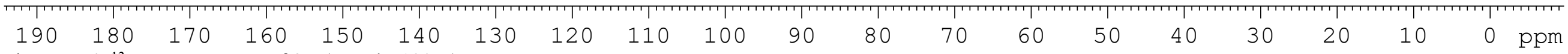

Figure S74. ${ }^{13} \mathrm{C}$ NMR spectra of $\mathbf{3 L}\left(\mathrm{CDCl}_{3}, 100 \mathrm{M}\right)$.

S-92 
<smiles>O=C(OC1CCCCCCCCCCC1)c1ccc(C(=O)ON2C(=O)c3ccccc3C2=O)cc1</smiles>
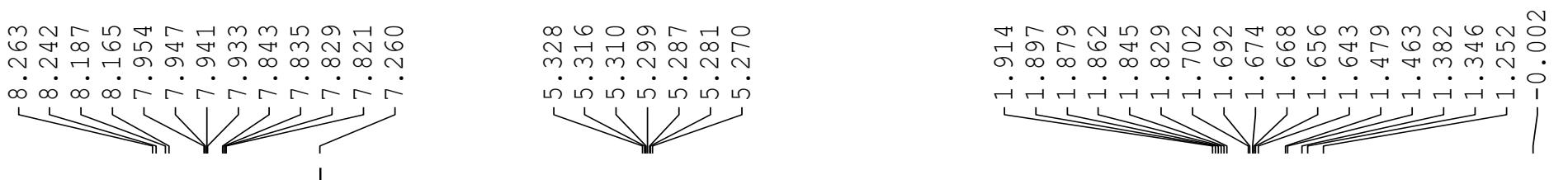

BM

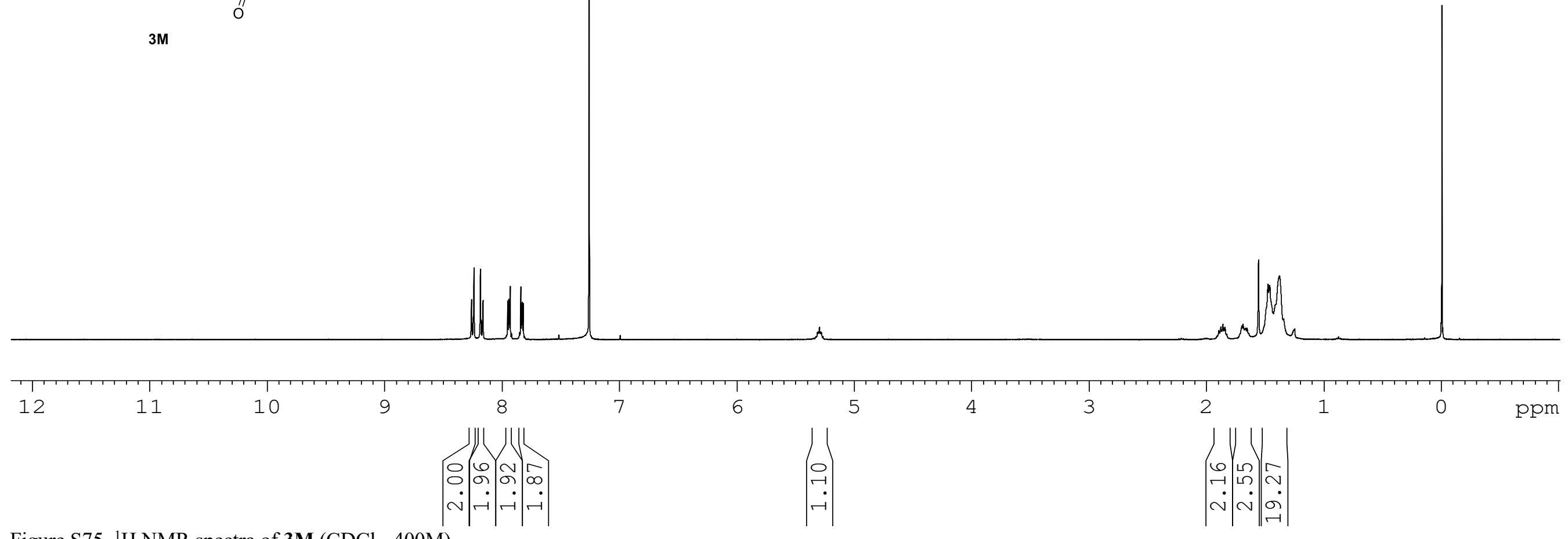

Figure S75. ${ }^{1} \mathrm{H}$ NMR spectra of $\mathbf{3 M}\left(\mathrm{CDCl}_{3}, 400 \mathrm{M}\right)$.

S-93 

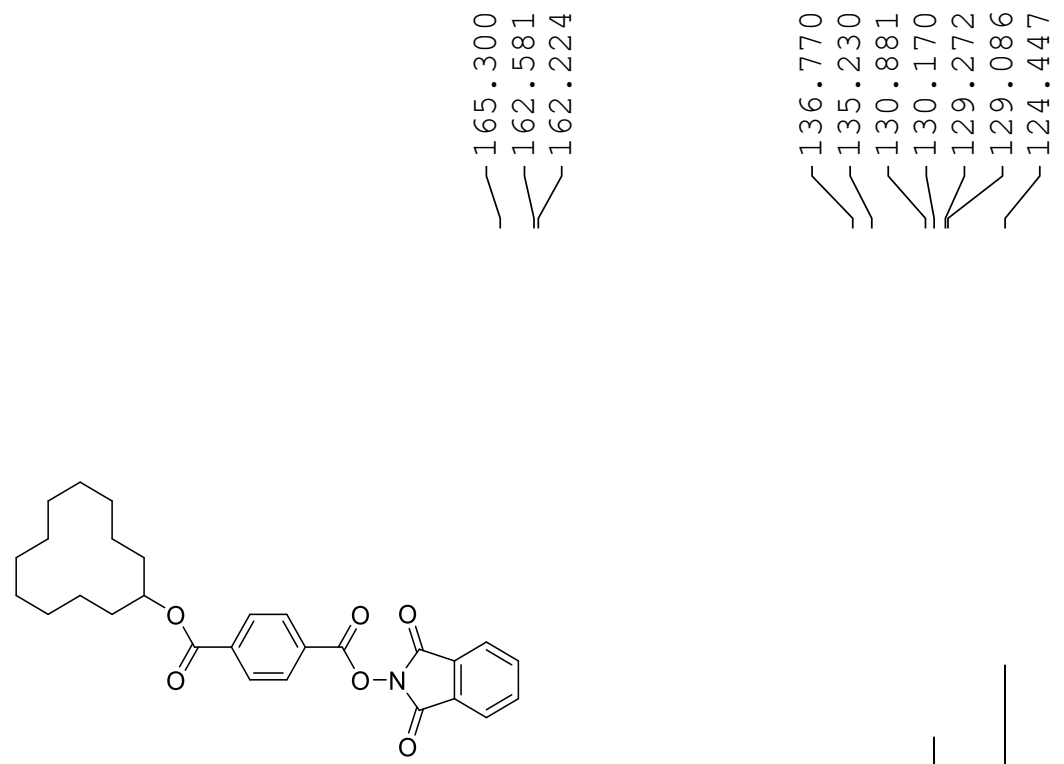

$3 \mathrm{M}$

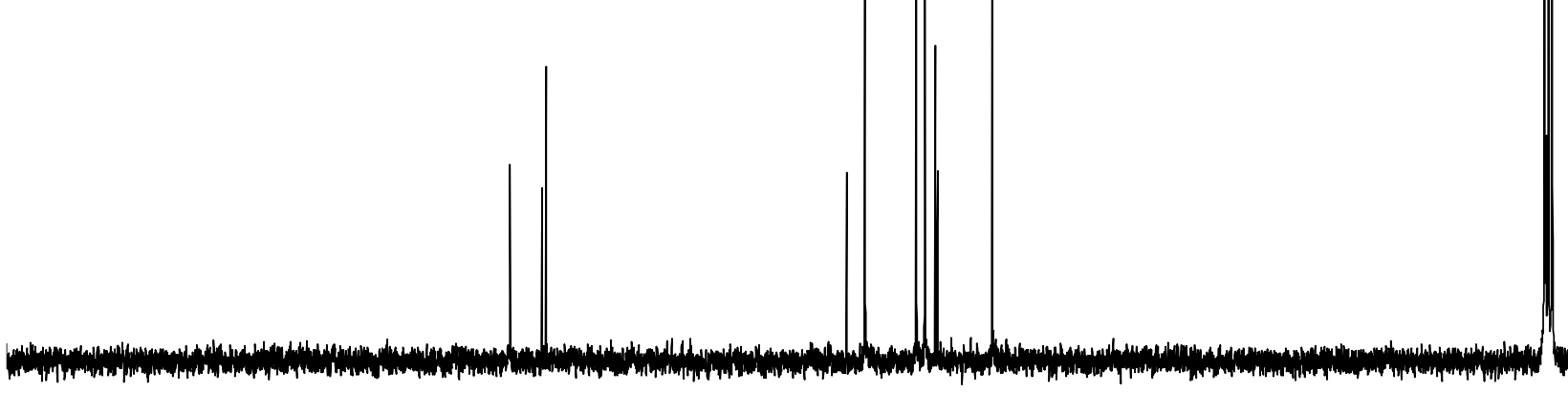

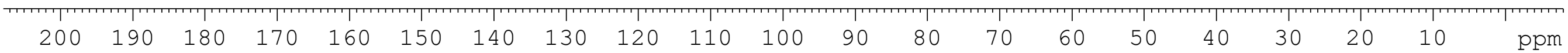
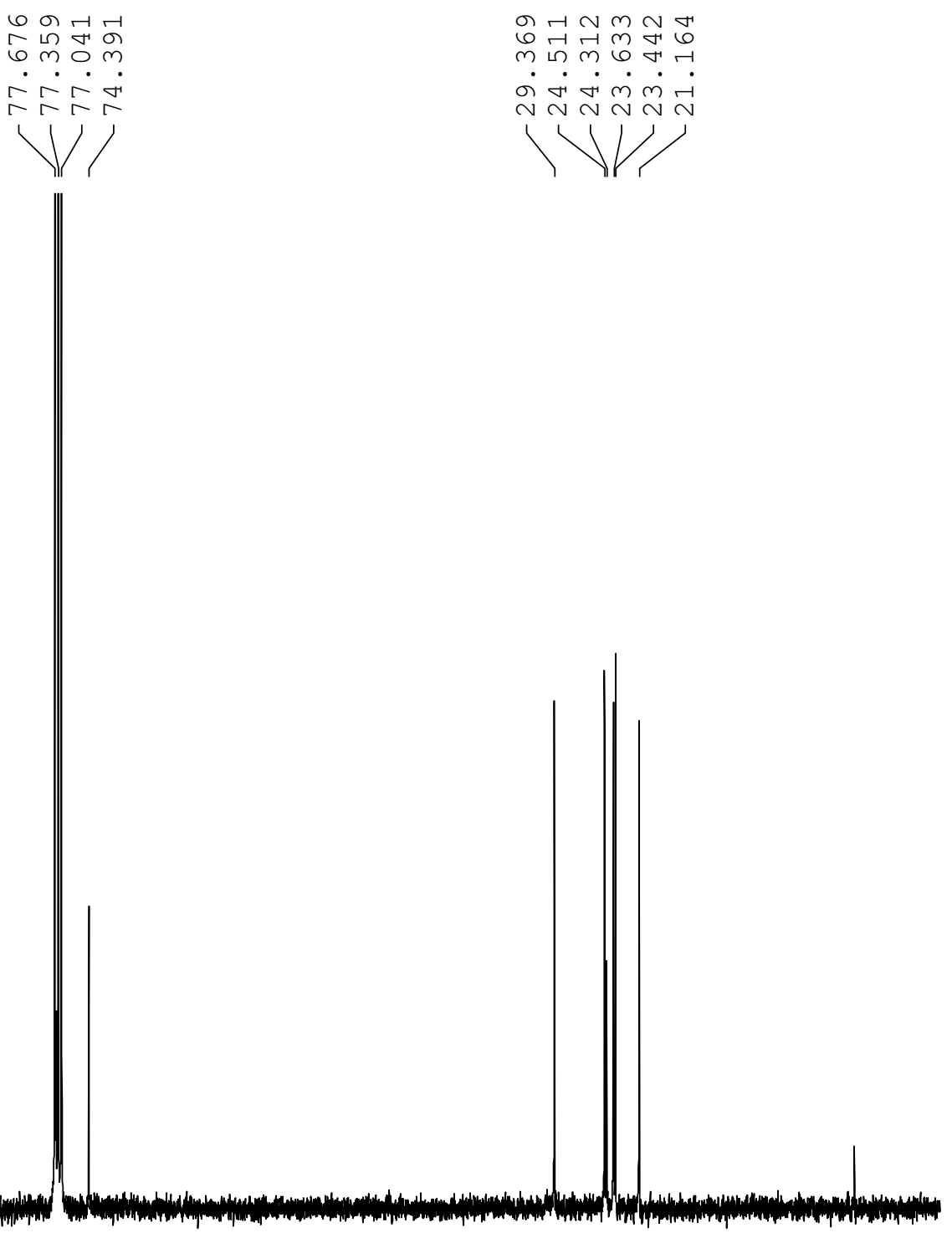

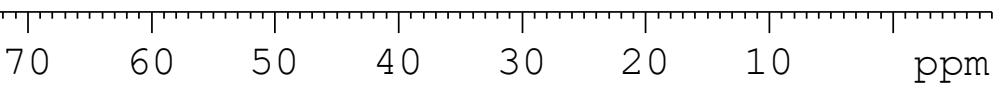

Figure S76. ${ }^{13} \mathrm{C}$ NMR spectra of $\mathbf{3 M}\left(\mathrm{CDCl}_{3}, 100 \mathrm{M}\right)$.

S-94 


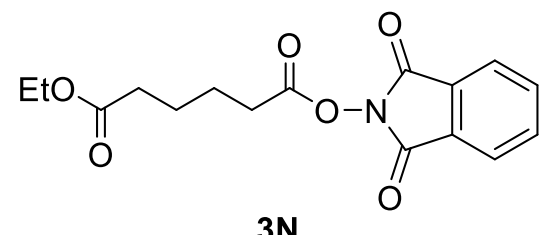

$3 N$
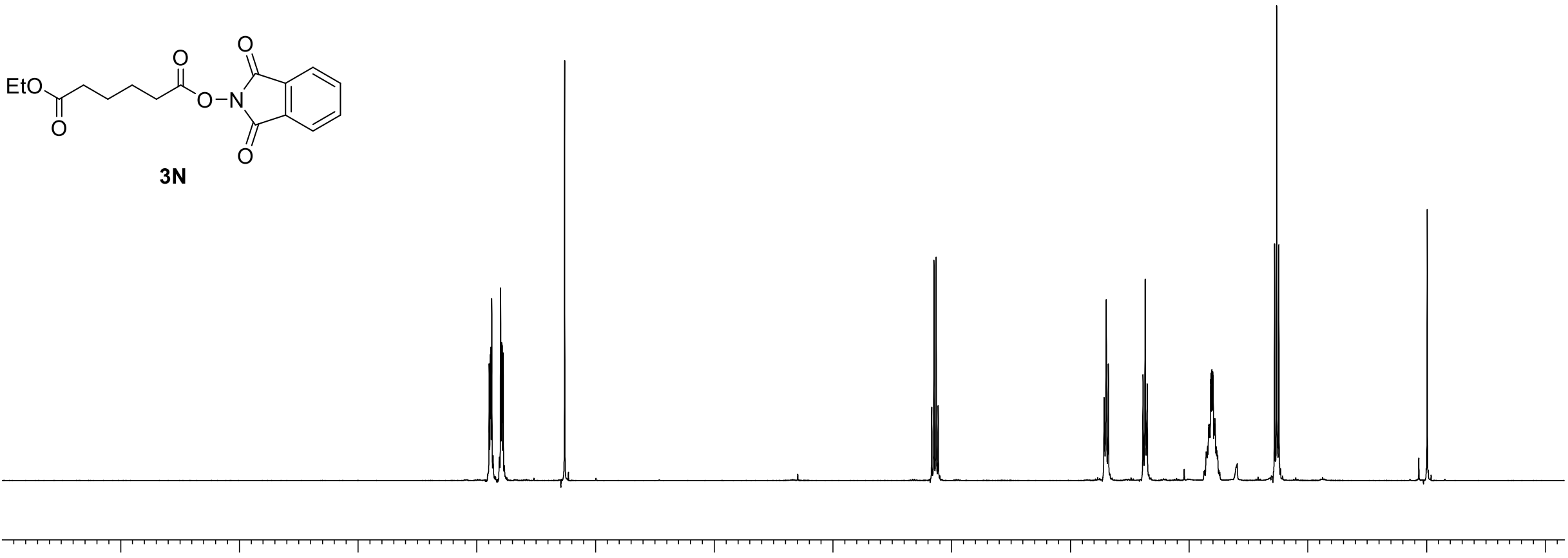

10

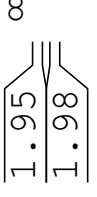

6

Figure S77. ${ }^{1} \mathrm{H}$ NMR spectra of $\mathbf{3 N}\left(\mathrm{CDCl}_{3}, 400 \mathrm{M}\right)$. 

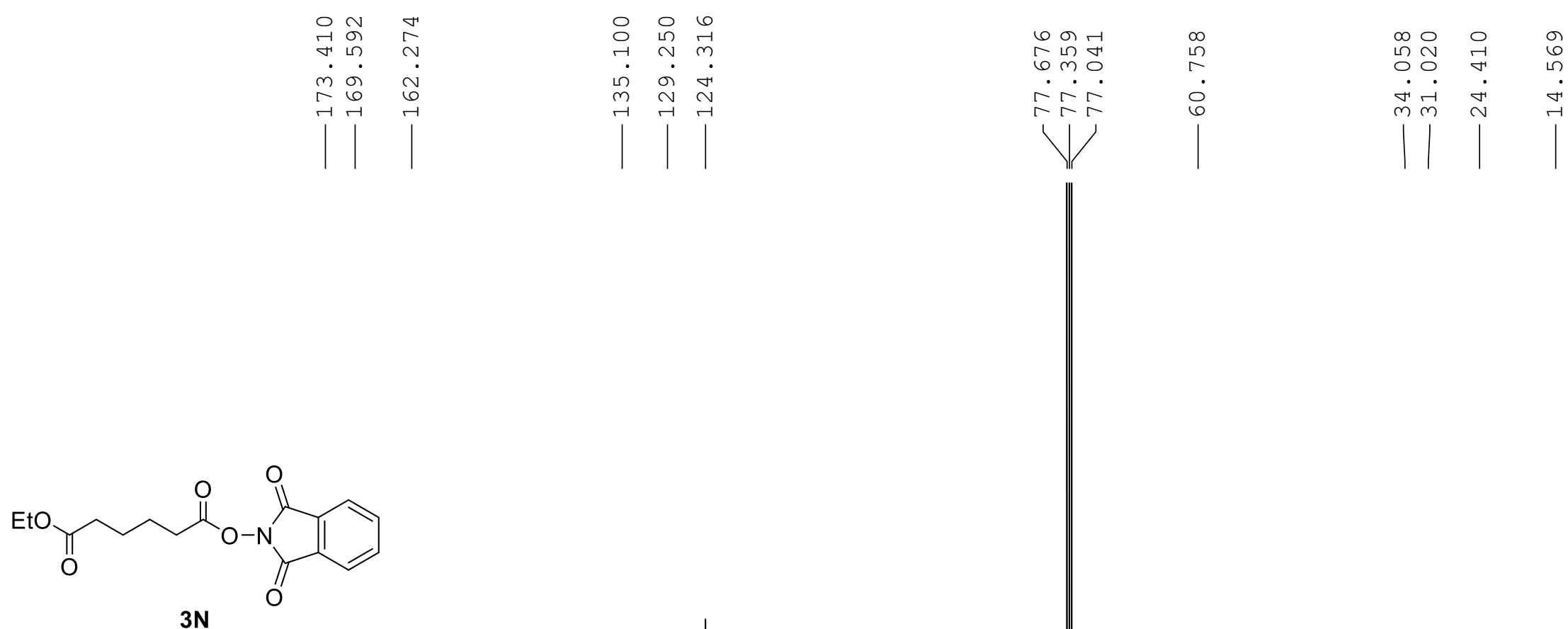

$3 N$

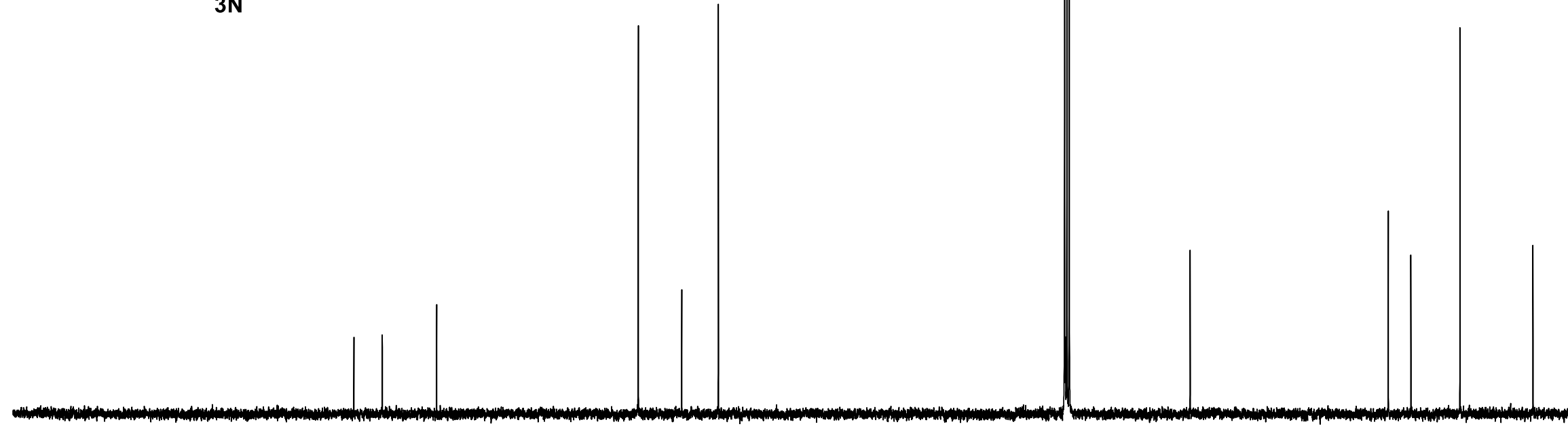

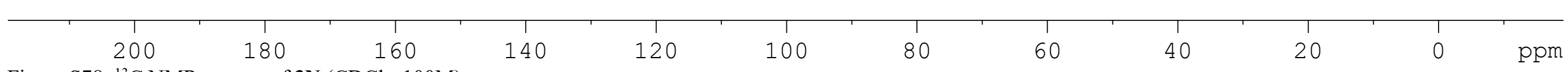

Figure $\mathrm{S} 78 .{ }^{13} \mathrm{C}$ NMR spectra of $\mathbf{3 N}\left(\mathrm{CDCl}_{3}, 100 \mathrm{M}\right)$. 
$\mathrm{Cl}$<smiles>O=C(CCCCCOC(=O)c1ccc(Cl)cc1)ON1C(=O)c2ccccc2C1=O</smiles>

30
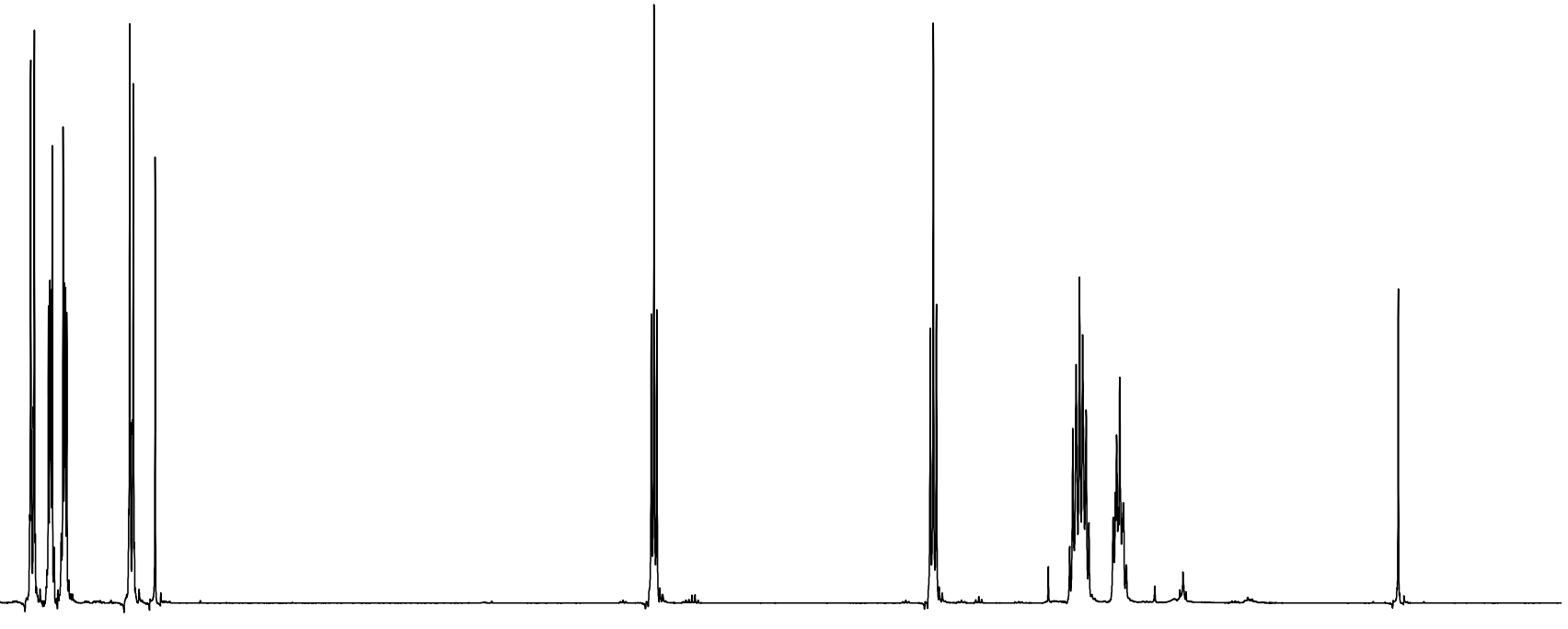

T'

11

10

Figure S79. ${ }^{1} \mathrm{H}$ NMR spectra of $\mathbf{3 O}\left(\mathrm{CDCl}_{3}, 400 \mathrm{M}\right)$.

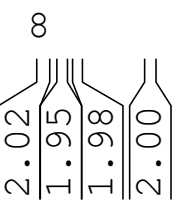

7

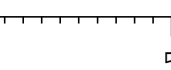

5
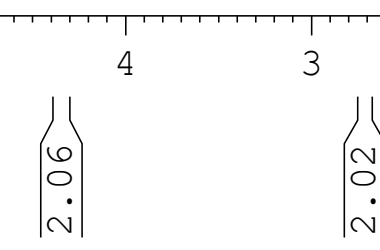

2

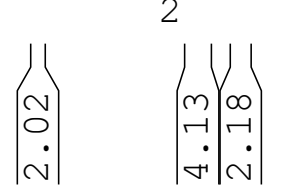

1

$0 \quad$ ppm

0
0
$ن$ 

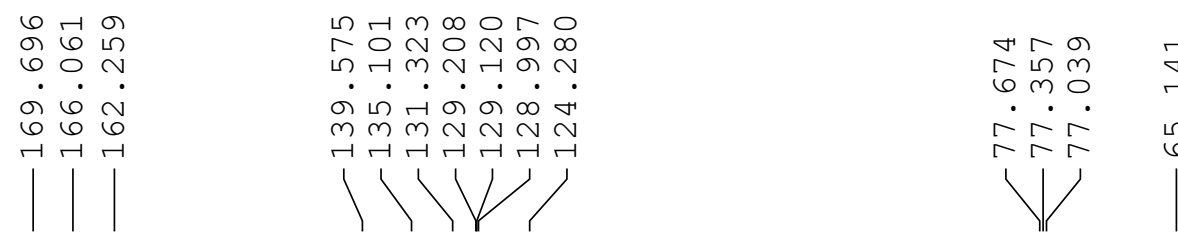

$\begin{array}{lll}-1 & 0 & 0 \\ 0 & \pi & 0\end{array}$

$\dot{-} \dot{\infty} \dot{\infty} \dot{\sigma}$

111

$\mathrm{Cl}$

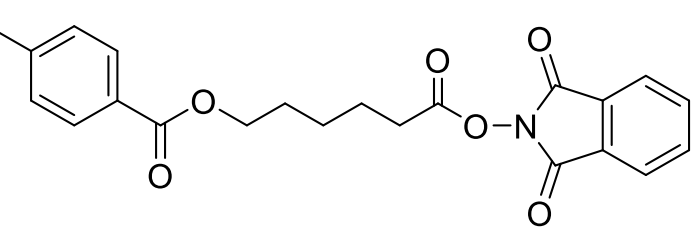

30
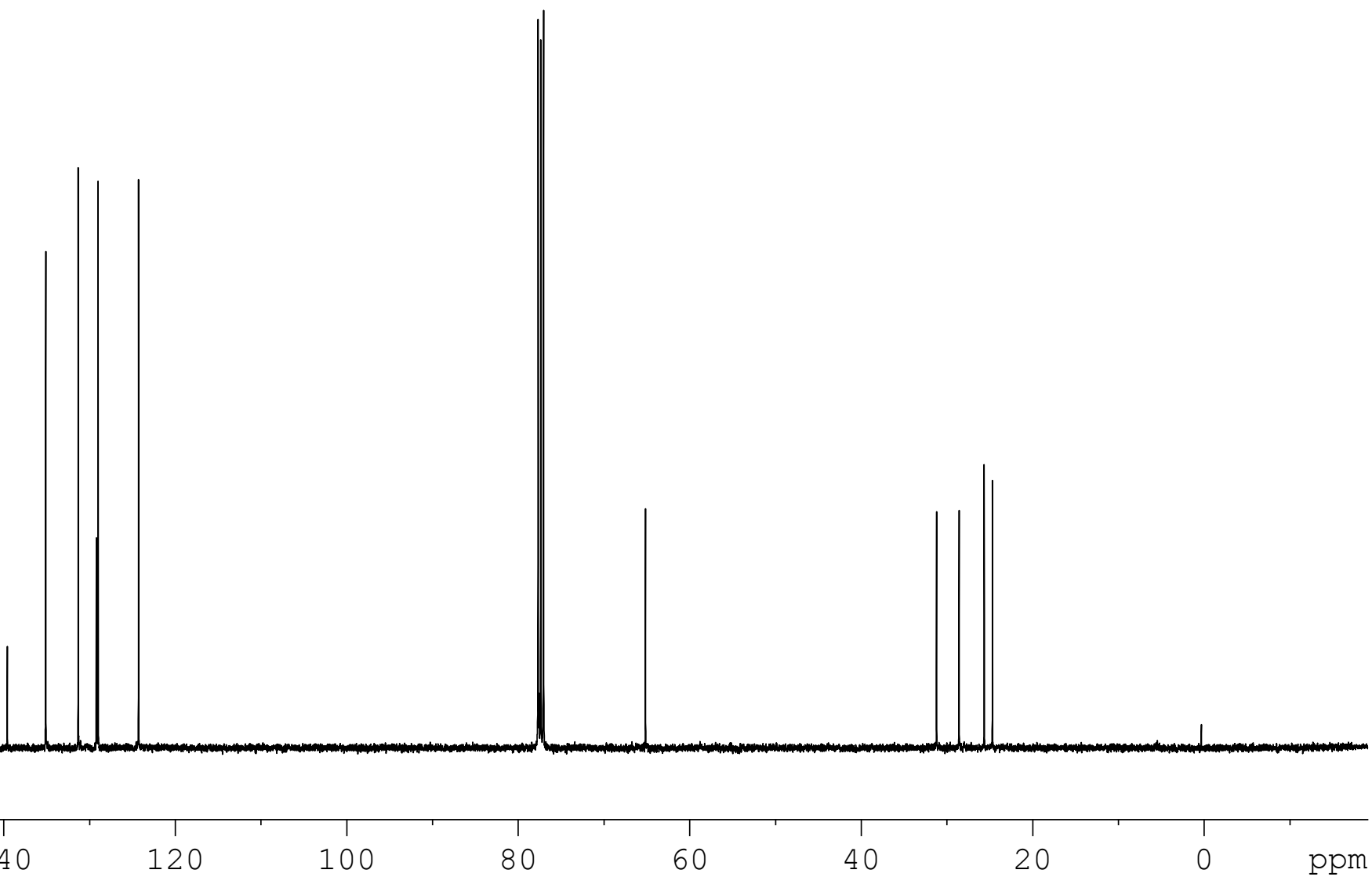

Figure $\mathrm{S} 80 .{ }^{13} \mathrm{C}$ NMR spectra of $\mathbf{3 0}\left(\mathrm{CDCl}_{3}, 100 \mathrm{M}\right)$. 


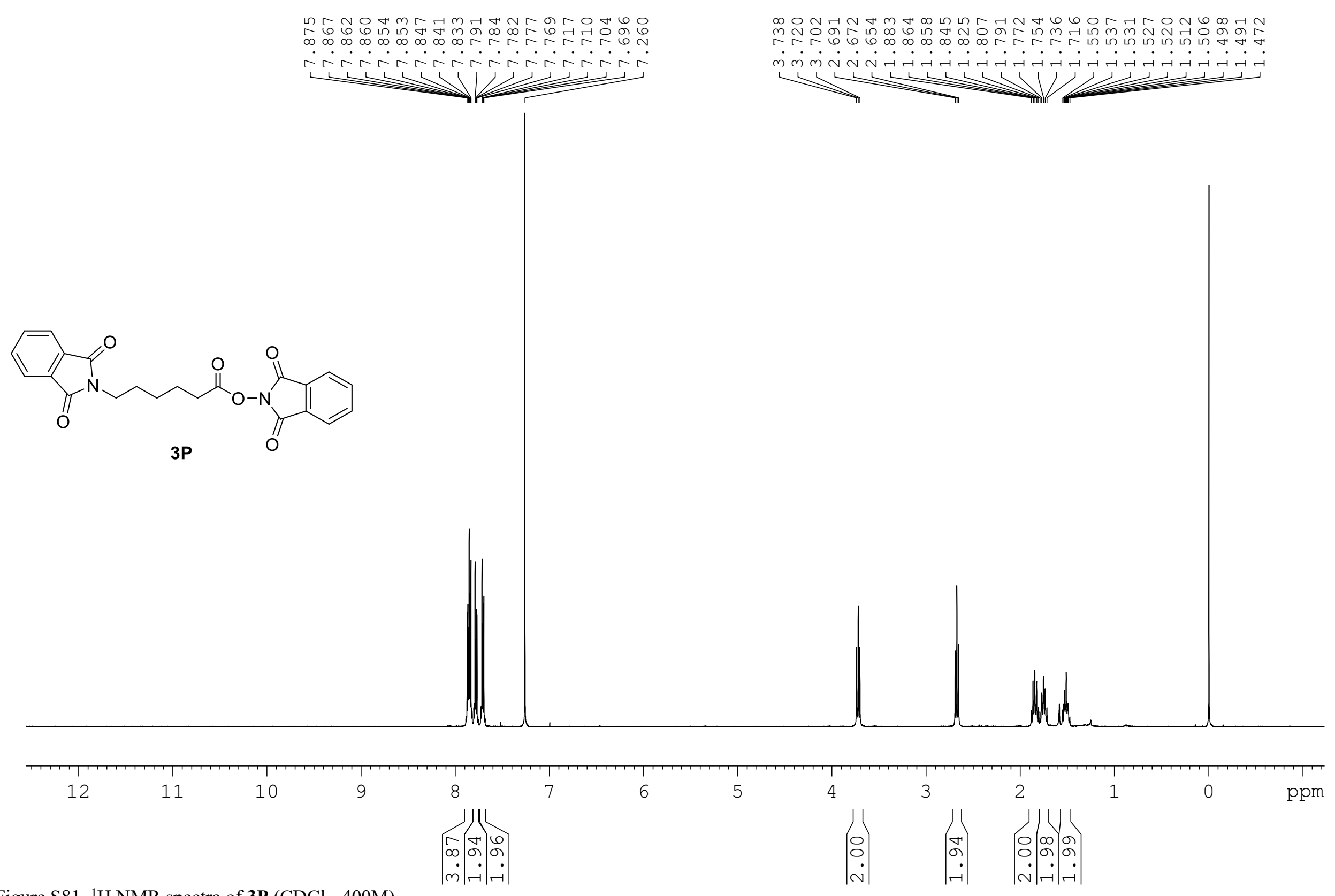




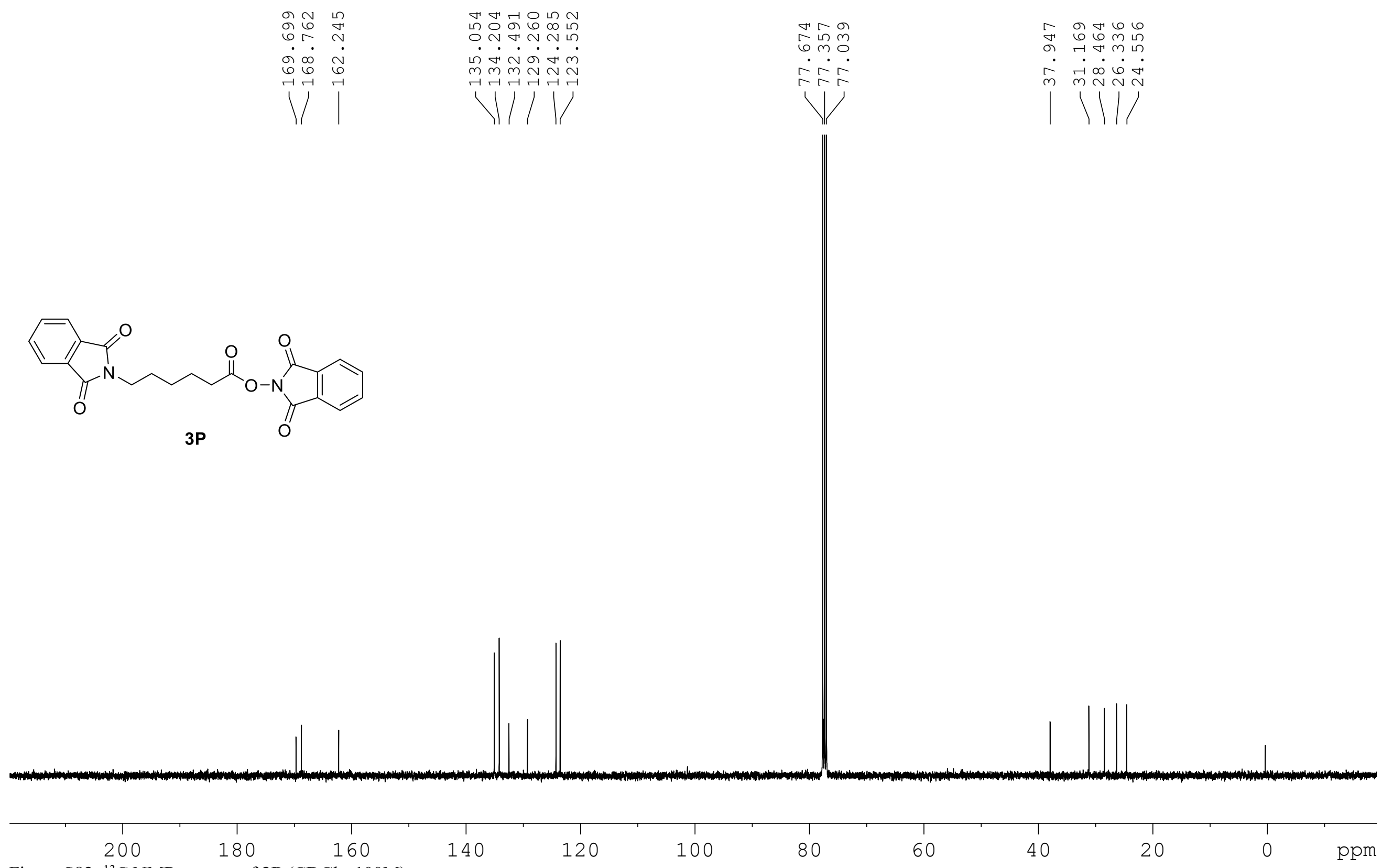

Figure $\mathrm{S} 82 .{ }^{13} \mathrm{C}$ NMR spectra of $\mathbf{3 P}\left(\mathrm{CDCl}_{3}, 100 \mathrm{M}\right)$. 


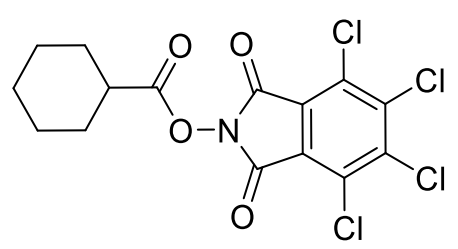

$3 \mathbf{Q}$

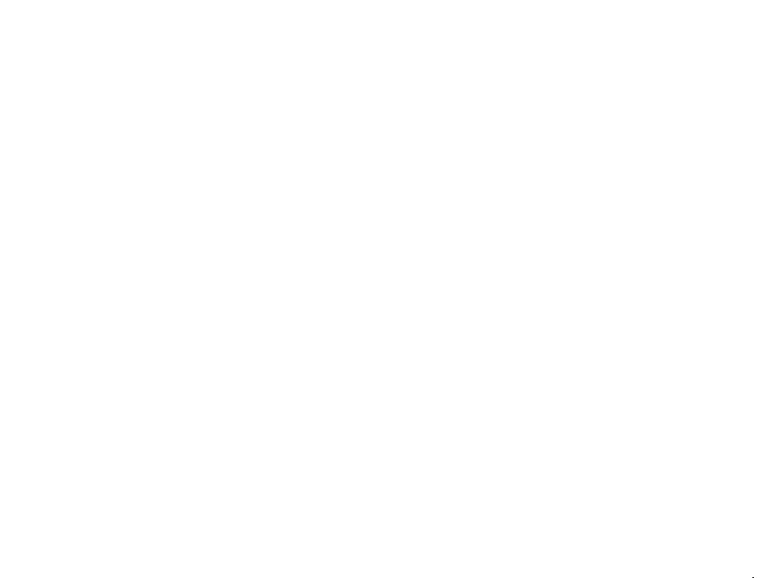

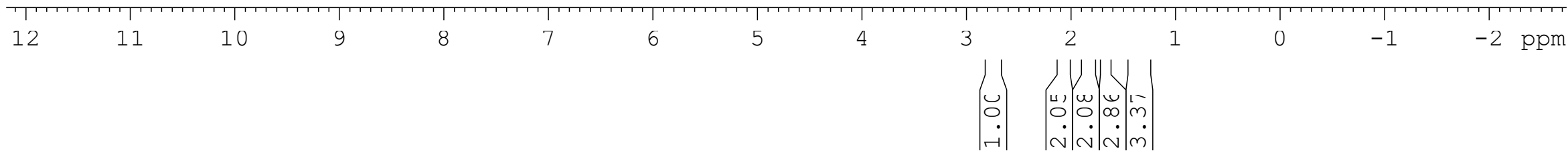

Figure S83. ${ }^{1} \mathrm{H}$ NMR spectra of $\mathbf{3 Q}\left(\mathrm{CDCl}_{3}, 400 \mathrm{M}\right)$. 


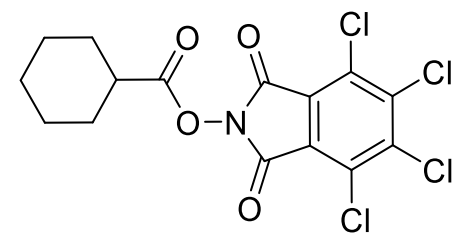

$3 Q$
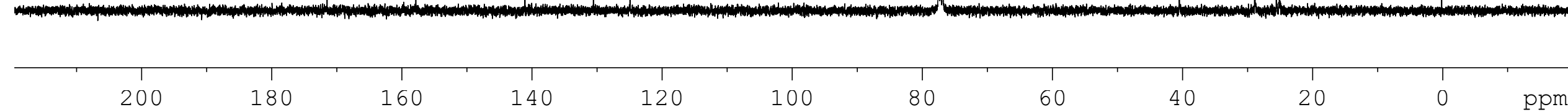

Figure S84. ${ }^{13} \mathrm{C}$ NMR spectra of $\mathbf{3 Q}\left(\mathrm{CDCl}_{3}, 100 \mathrm{M}\right)$. 


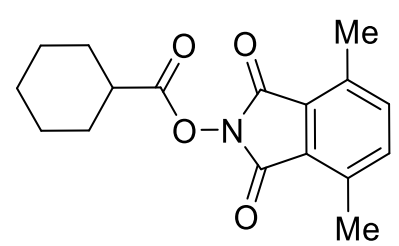

$3 \mathbf{R}$
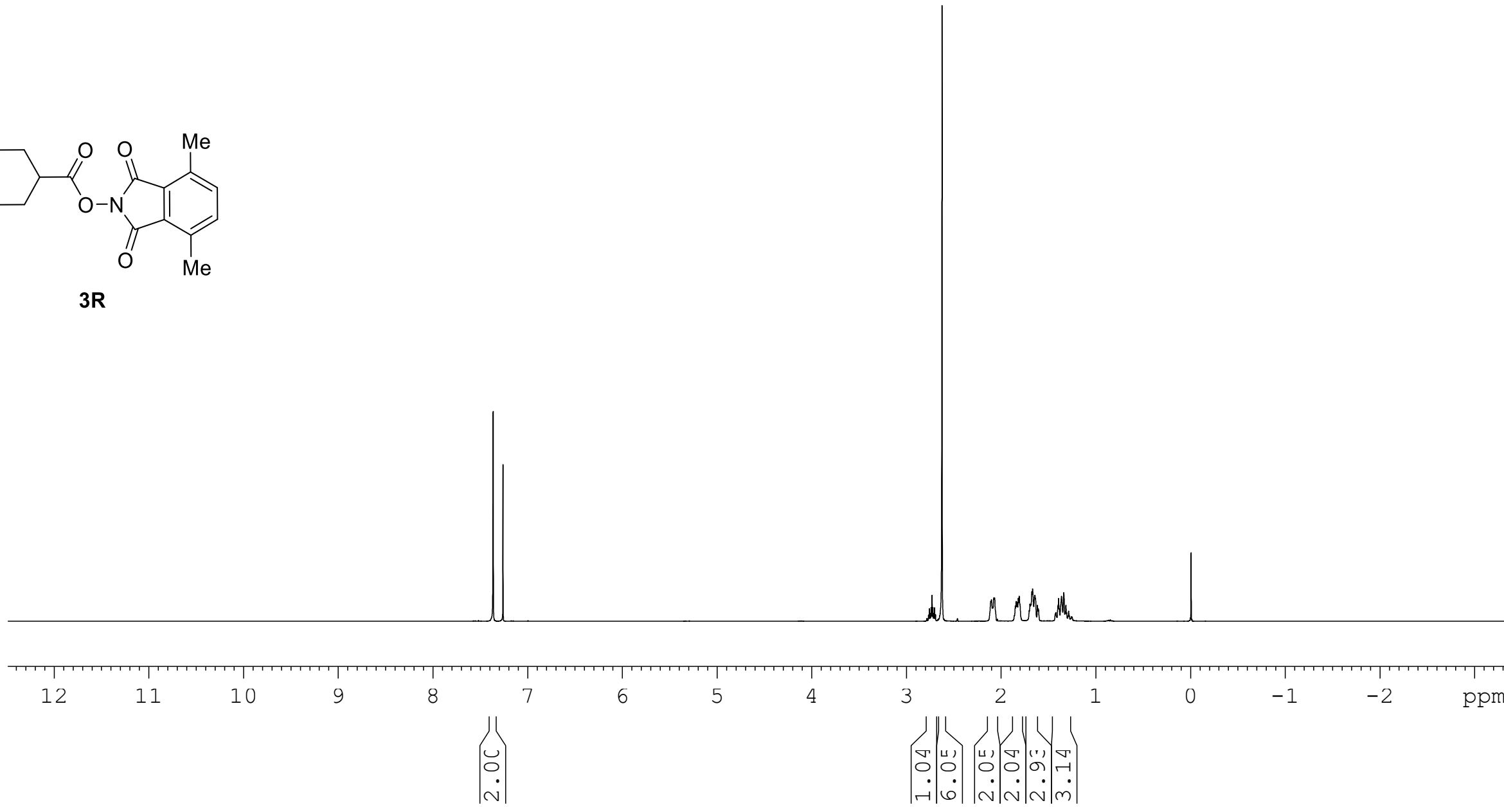

Figure S85. ${ }^{1} \mathrm{H}$ NMR spectra of $\mathbf{3 R}\left(\mathrm{CDCl}_{3}, 400 \mathrm{M}\right)$. 


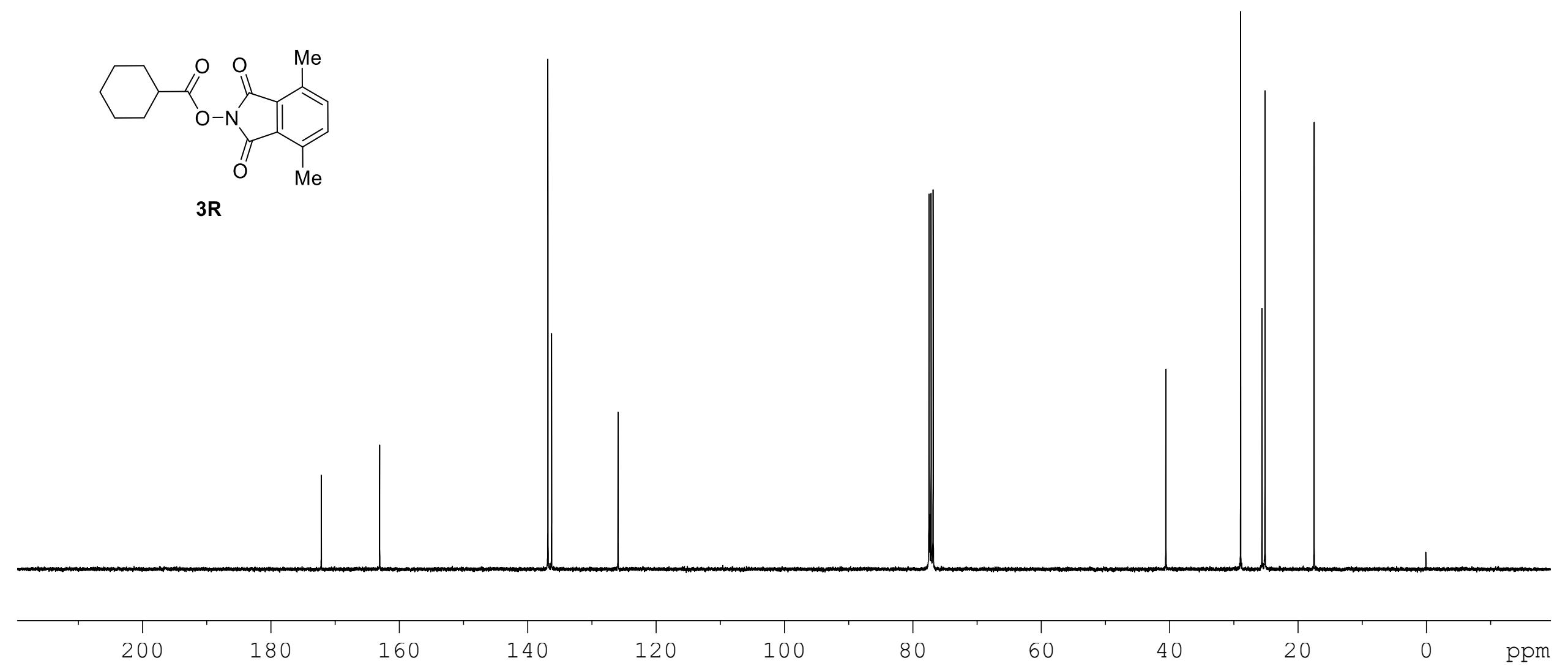

Figure S86. ${ }^{13} \mathrm{C}$ NMR spectra of $\mathbf{3 R}\left(\mathrm{CDCl}_{3}, 100 \mathrm{M}\right)$. 


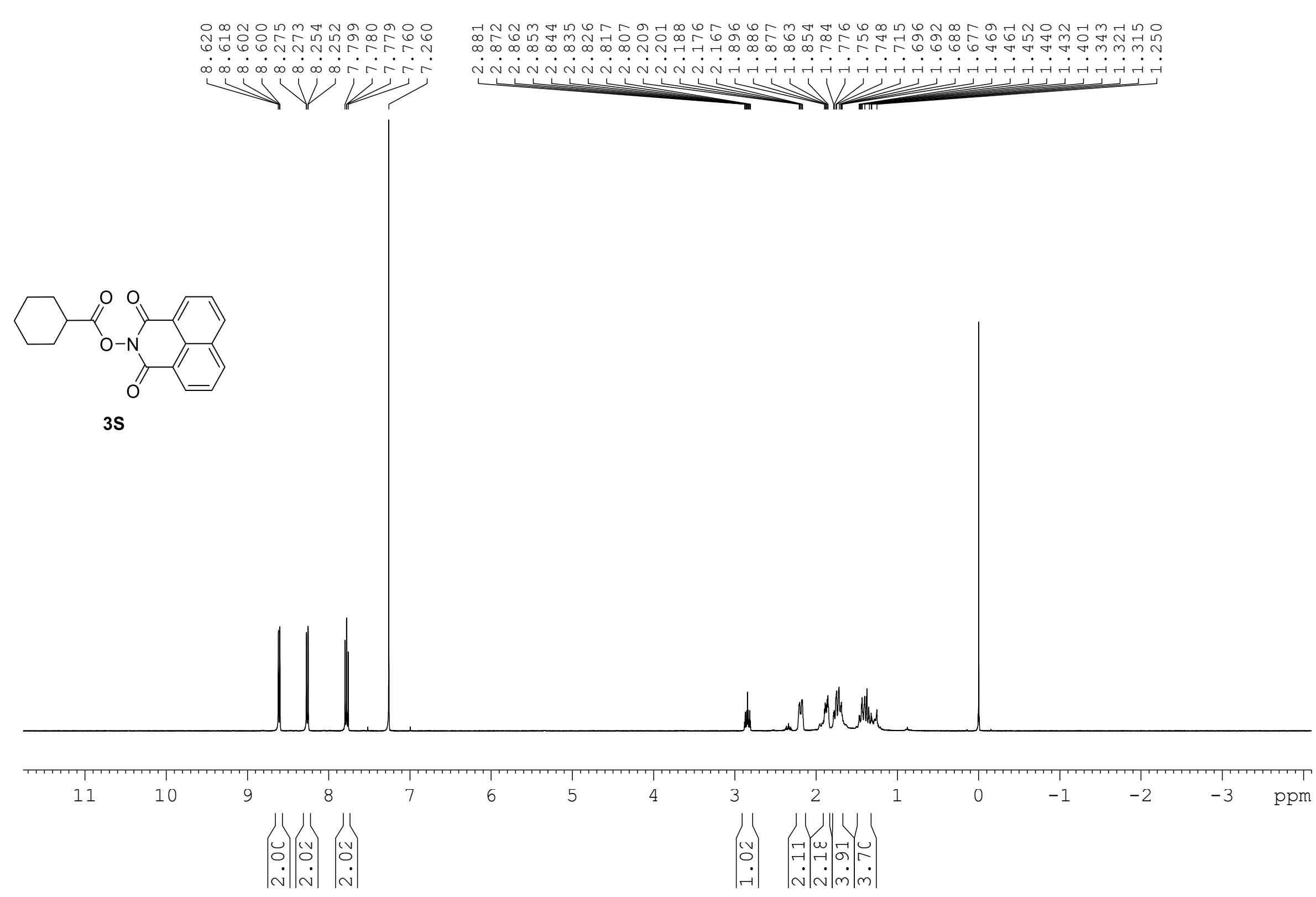

Figure S87. ${ }^{1} \mathrm{H}$ NMR spectra of $\mathbf{3 S}\left(\mathrm{CDCl}_{3}, 400 \mathrm{M}\right)$. 


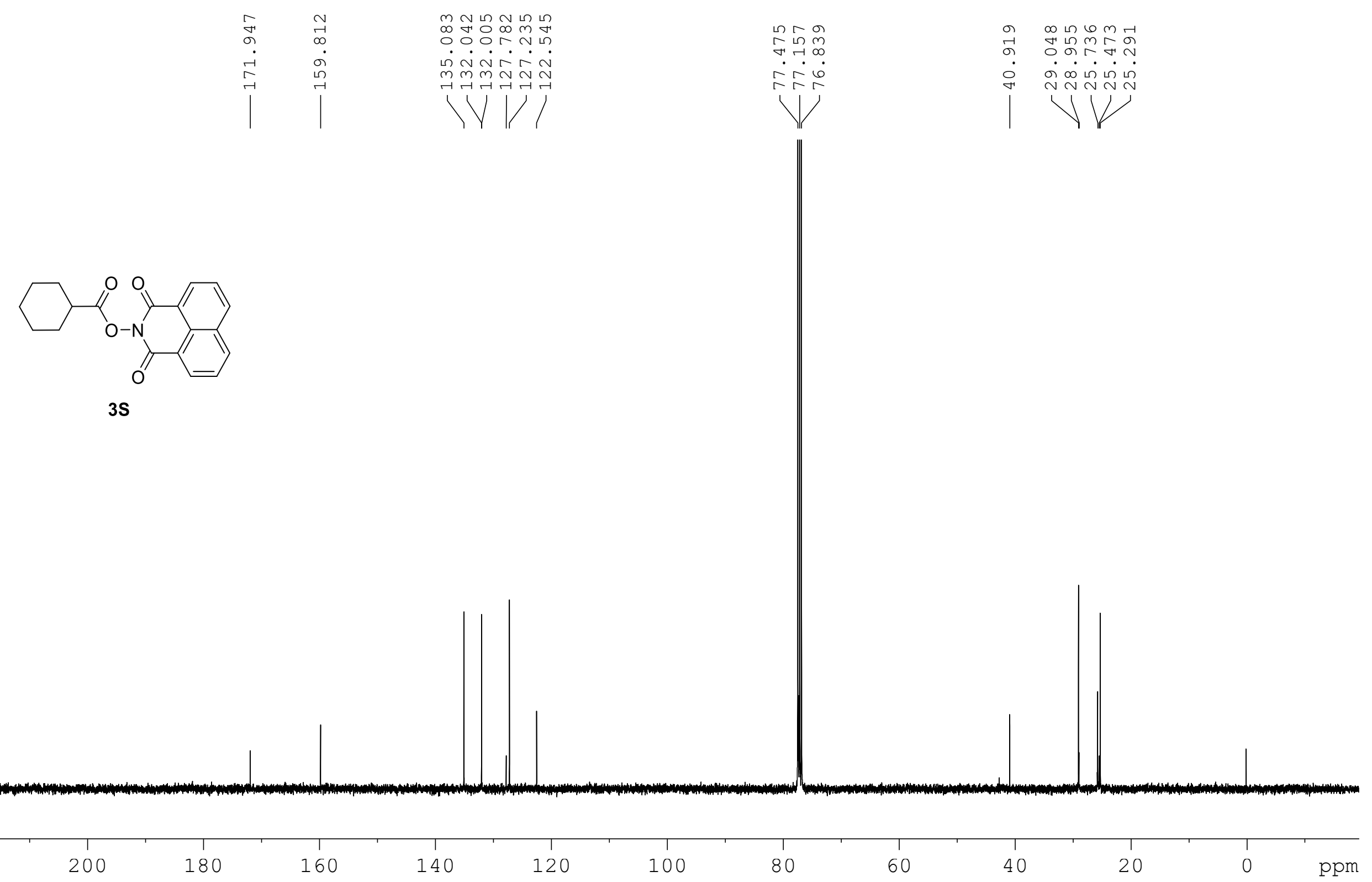

Figure S88. ${ }^{13} \mathrm{C}$ NMR spectra of $\mathbf{3 S}\left(\mathrm{CDCl}_{3}, 100 \mathrm{M}\right)$. 

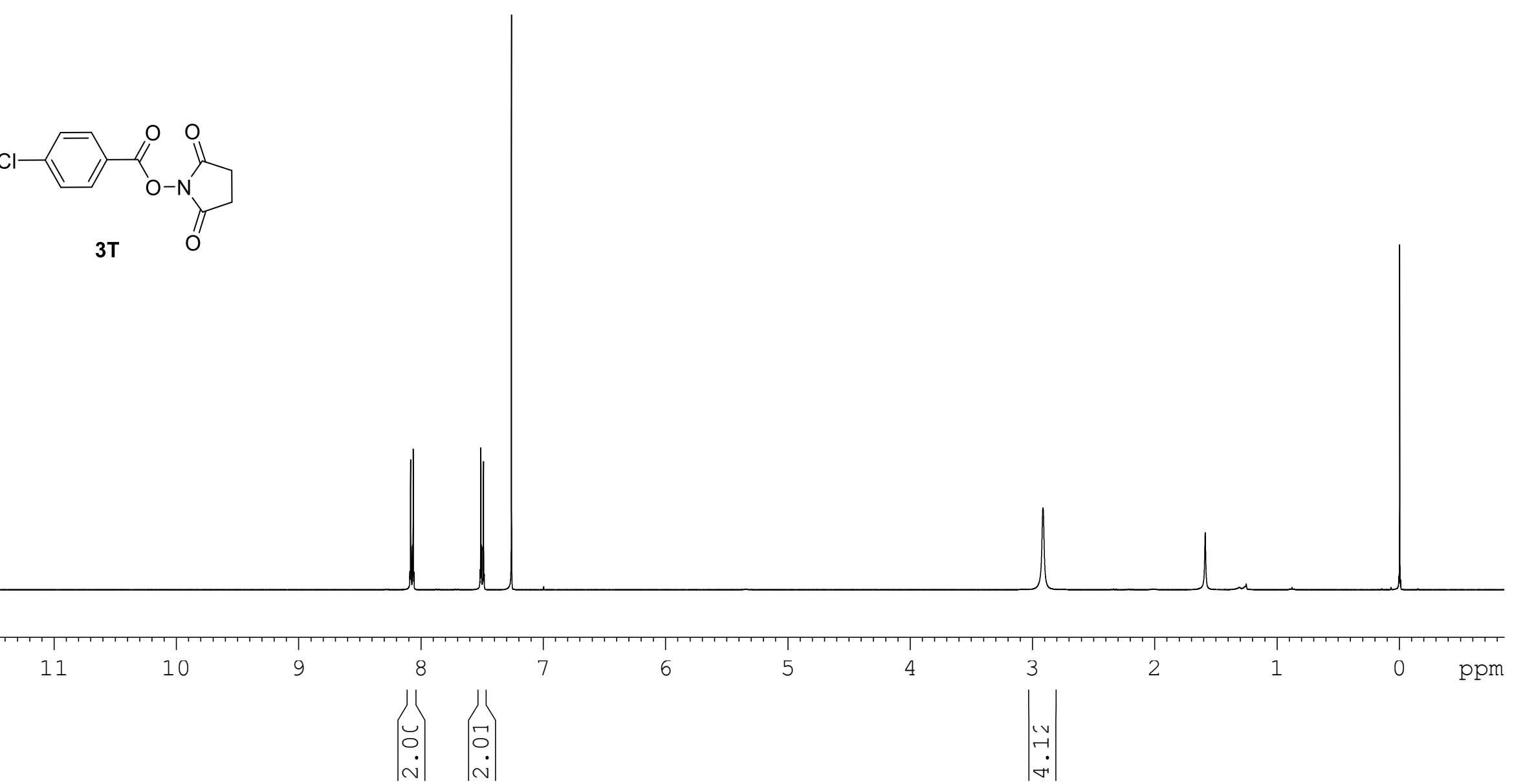

Figure S89. ${ }^{1} \mathrm{H}$ NMR spectra of 3T $\left(\mathrm{CDCl}_{3}, 400 \mathrm{M}\right)$. 


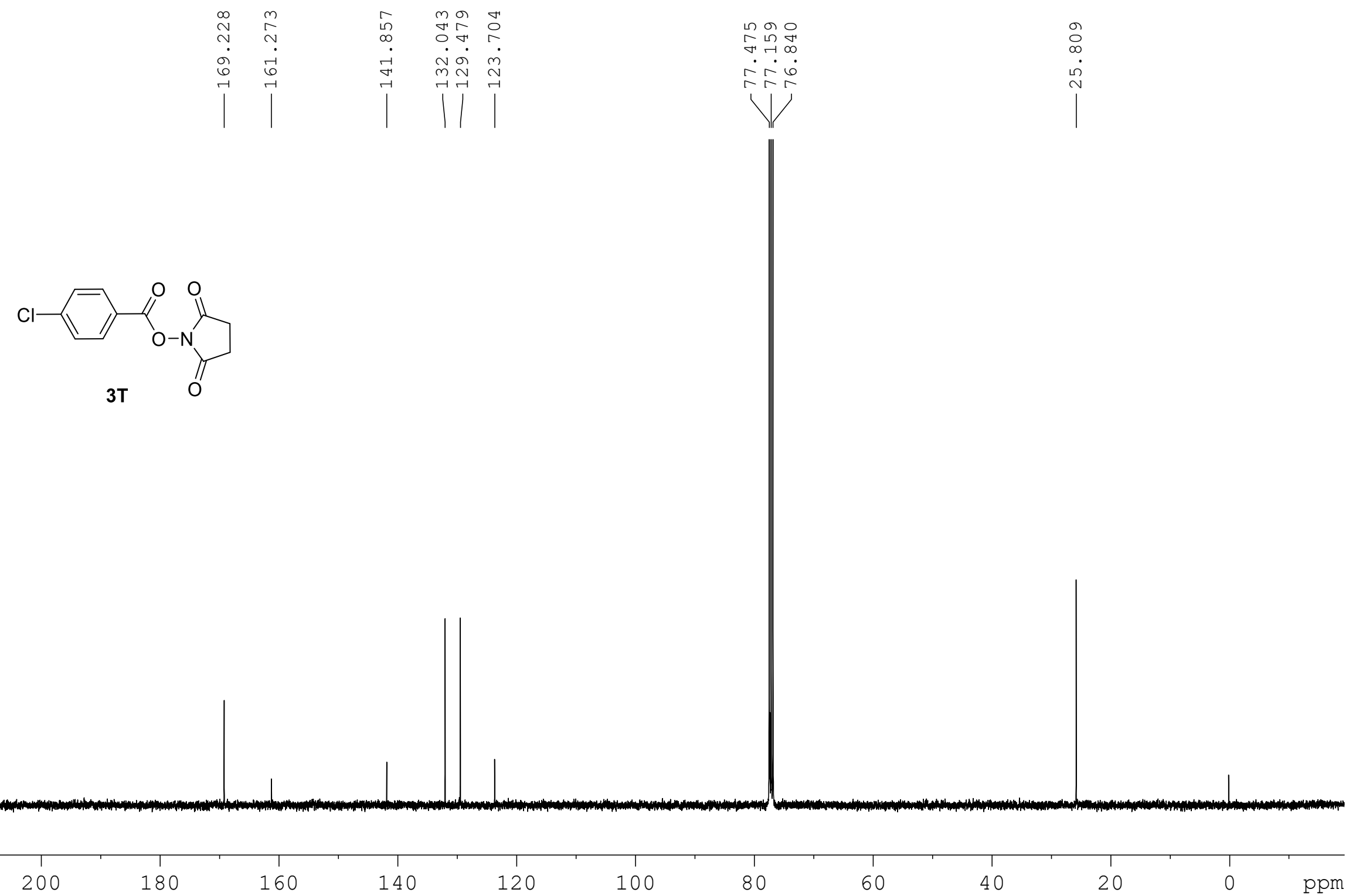

Figure S90. ${ }^{13} \mathrm{C}$ NMR spectra of $3 \mathrm{~T}\left(\mathrm{CDCl}_{3}, 100 \mathrm{M}\right)$. 


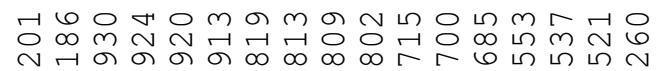

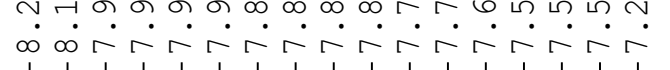<smiles>O=C(ON1C(=O)c2ccccc2C1=O)C(=O)c1ccccc1</smiles>

$5 a$
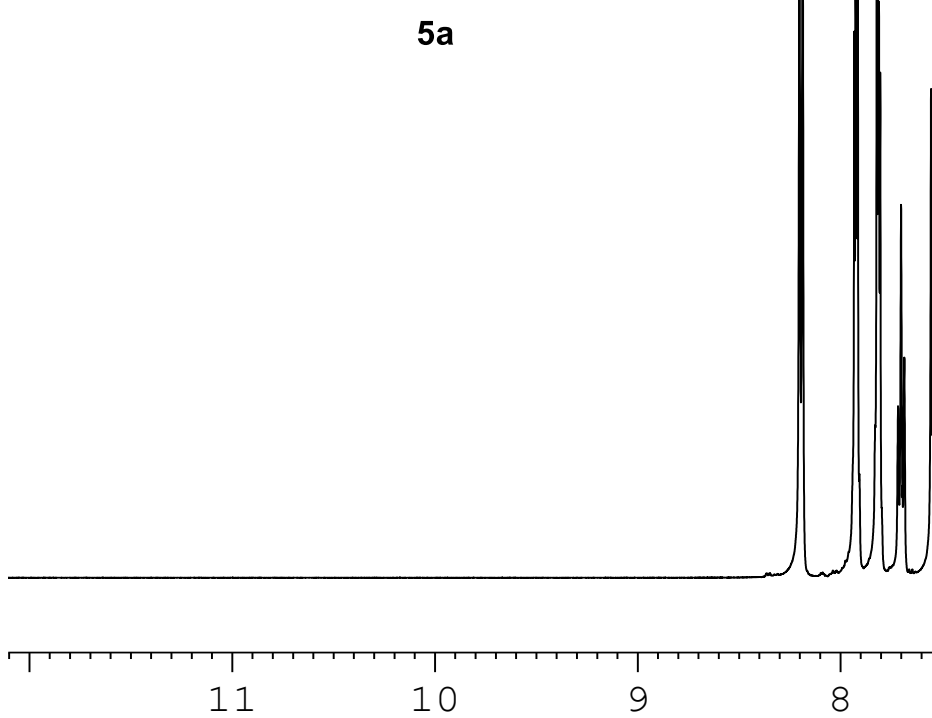

1
9

$8 \quad 7$

1
7

4

2

2

$1+1+1$

ppm

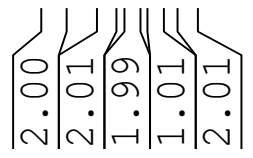

Figure S91. ${ }^{1} \mathrm{H}$ NMR spectra of $\mathbf{5 a}\left(\mathrm{CDCl}_{3}, 400 \mathrm{M}\right)$.

S-109 


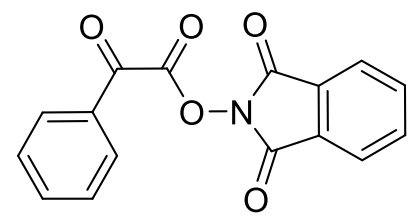

$5 a$
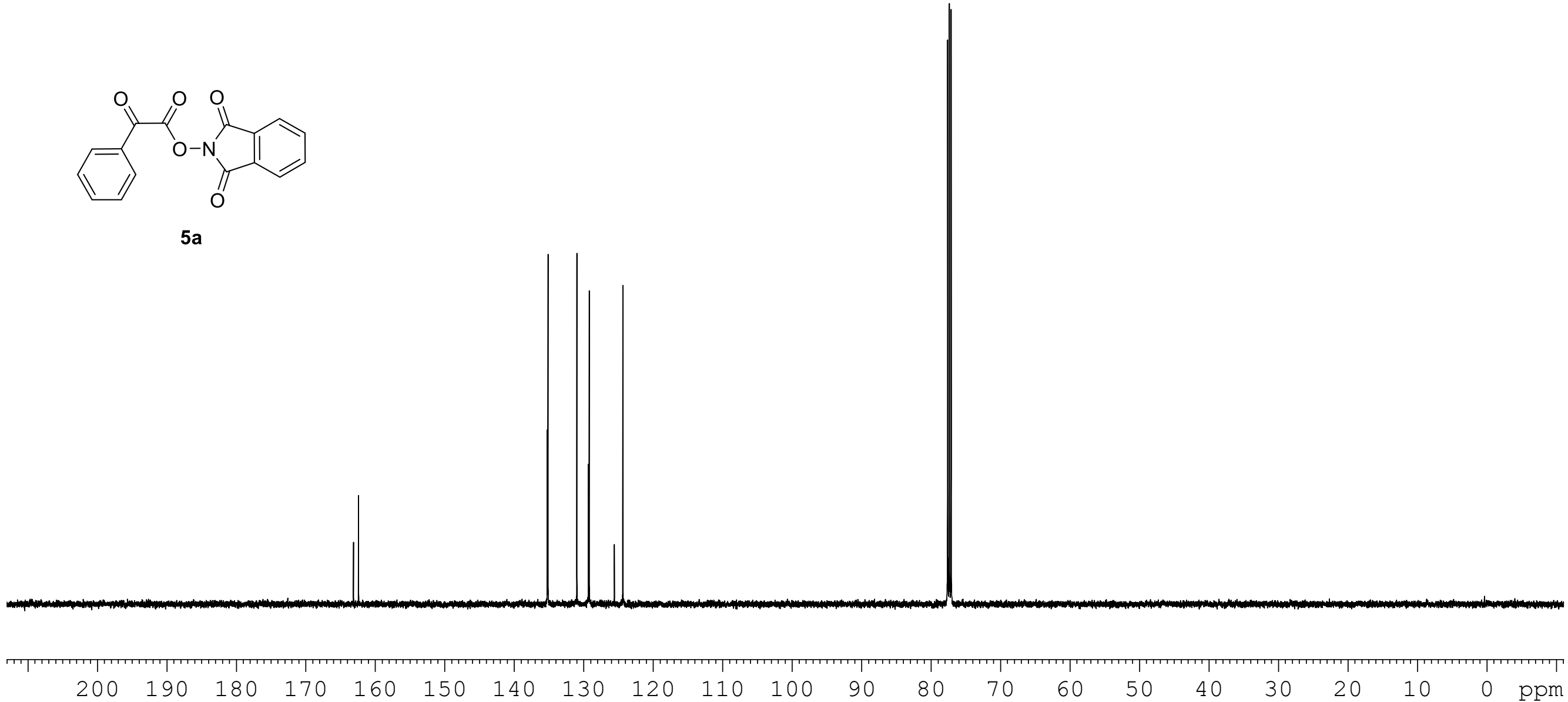

Figure S92. ${ }^{13} \mathrm{C}$ NMR spectra of $\mathbf{5 a}\left(\mathrm{CDCl}_{3}, 100 \mathrm{M}\right)$. 


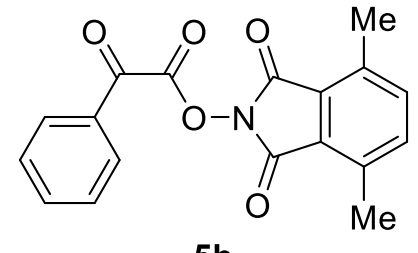

$5 b$

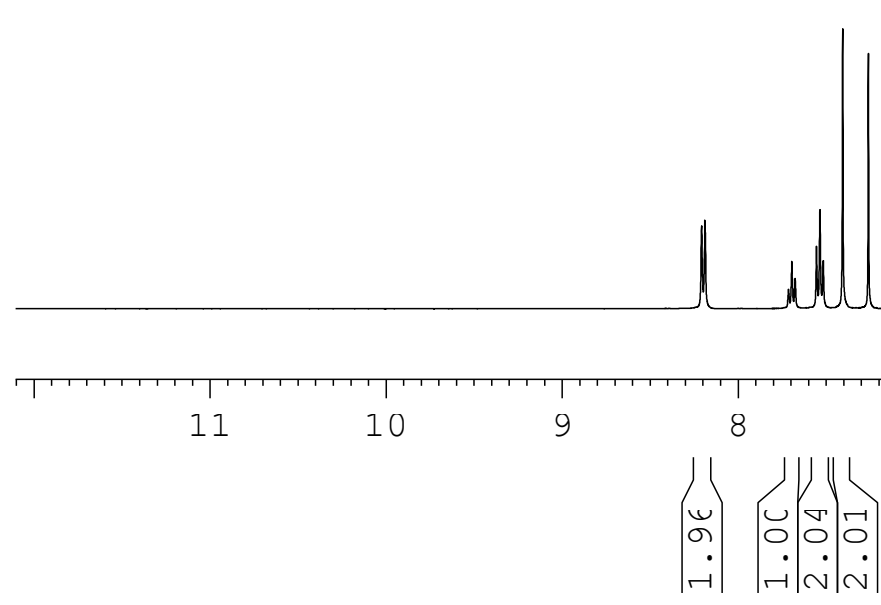

Figure S93. ${ }^{1} \mathrm{H}$ NMR spectra of $\mathbf{5 b}\left(\mathrm{CDCl}_{3}, 400 \mathrm{M}\right)$. 

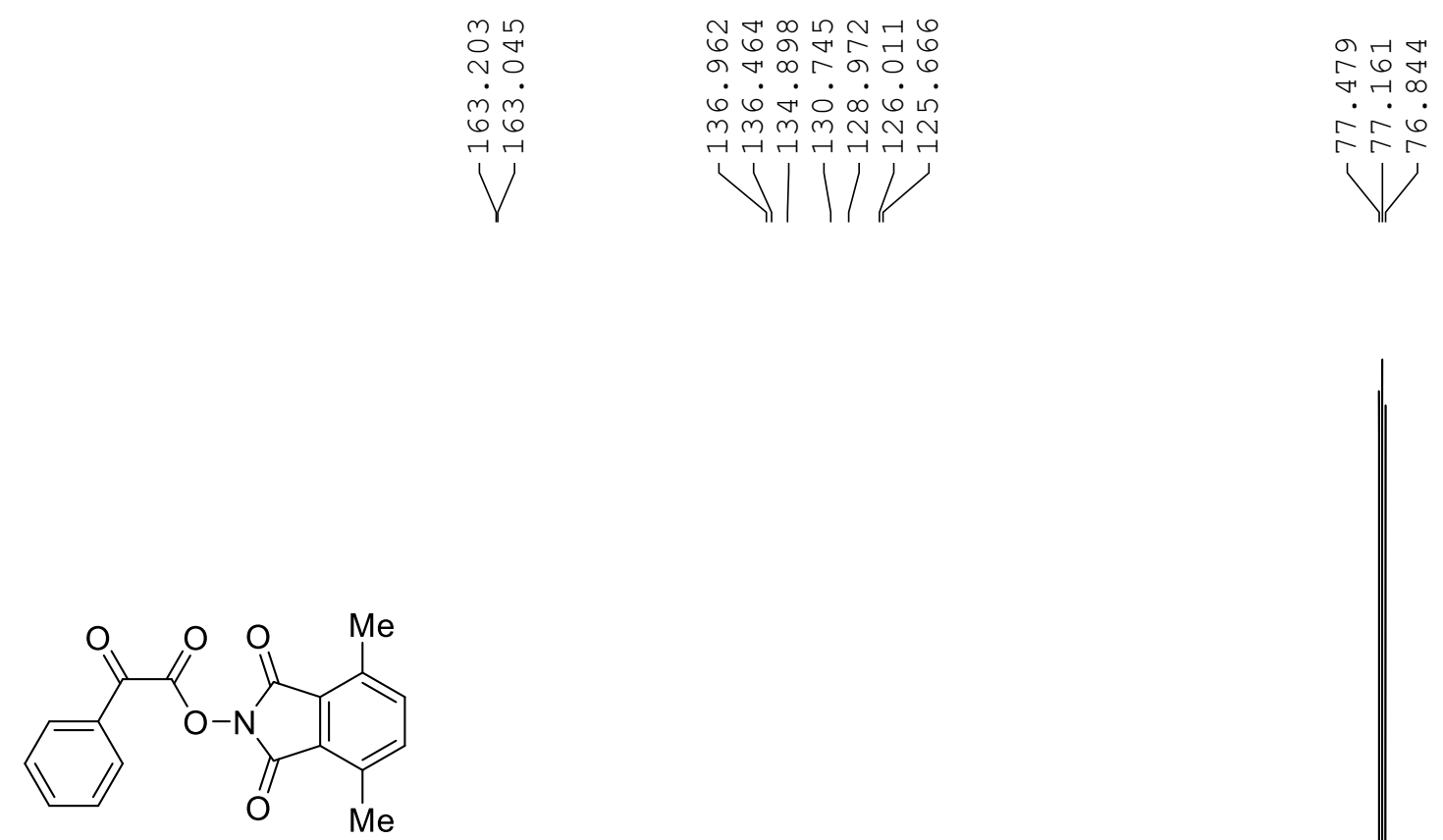

$5 b$

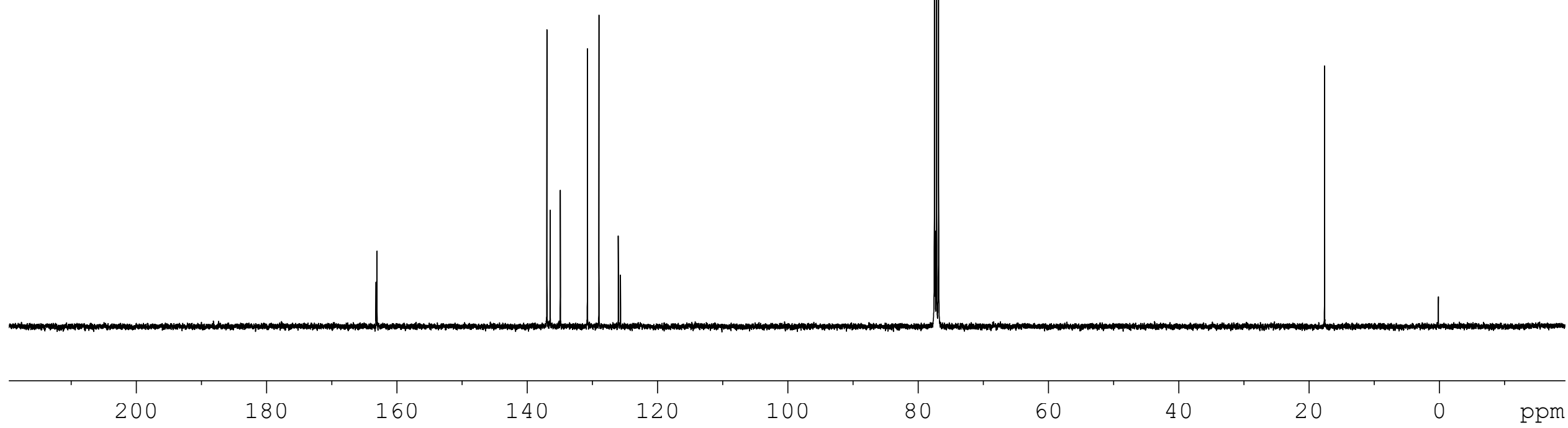

Figure S94. ${ }^{13} \mathrm{C}$ NMR spectra of $\mathbf{5 b}\left(\mathrm{CDCl}_{3}, 100 \mathrm{M}\right)$. 


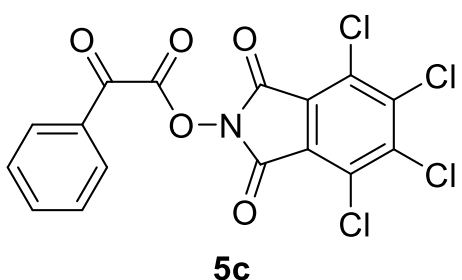

$5 c$
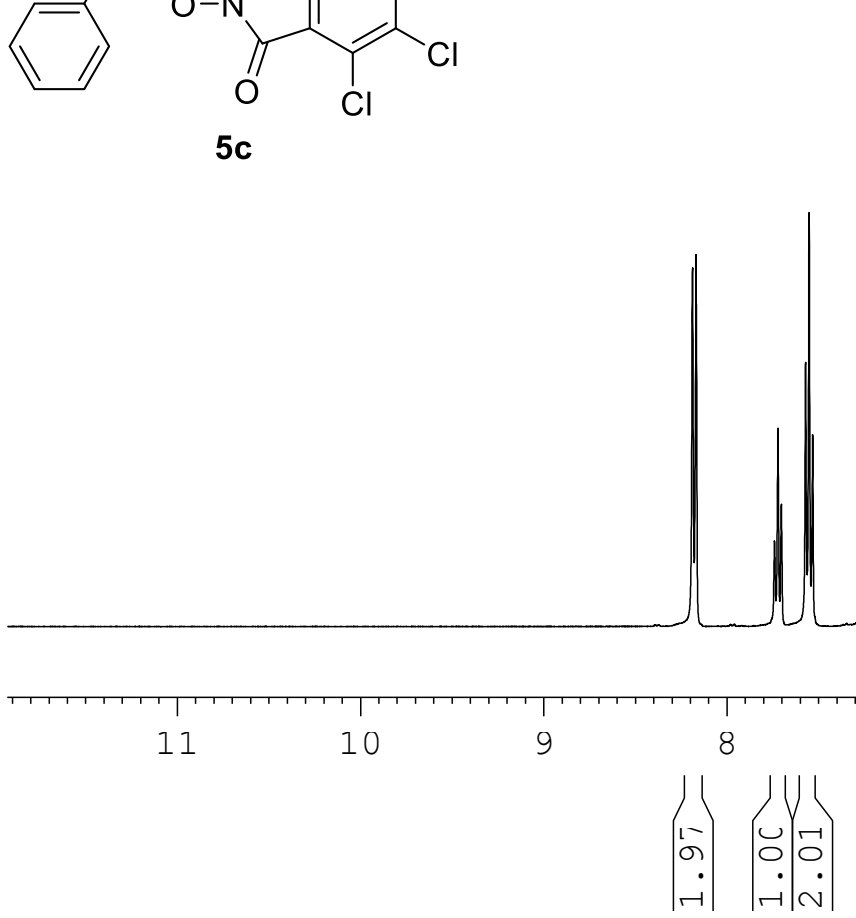

Figure S95. ${ }^{1} \mathrm{H}$ NMR spectra of $\mathbf{5} \mathbf{c}\left(\mathrm{CDCl}_{3}, 400 \mathrm{M}\right)$. 

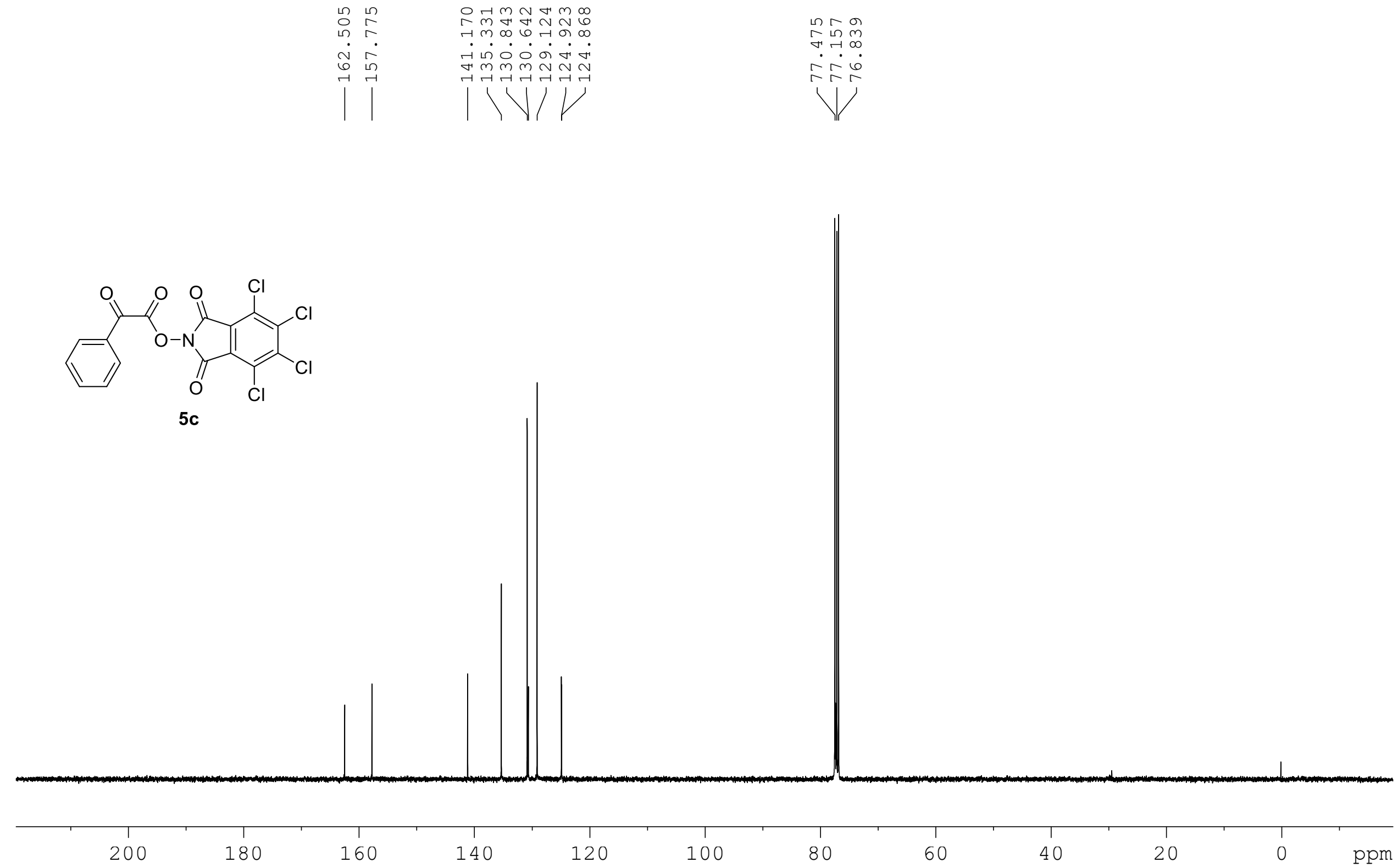

Figure $\mathrm{S} 96 .{ }^{13} \mathrm{C}$ NMR spectra of $\mathbf{5 c}\left(\mathrm{CDCl}_{3}, 100 \mathrm{M}\right)$. 


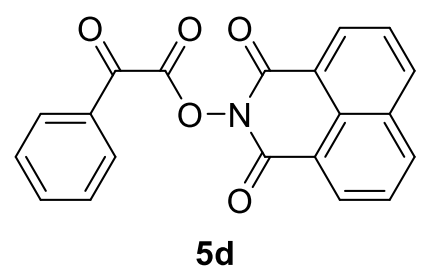

5d
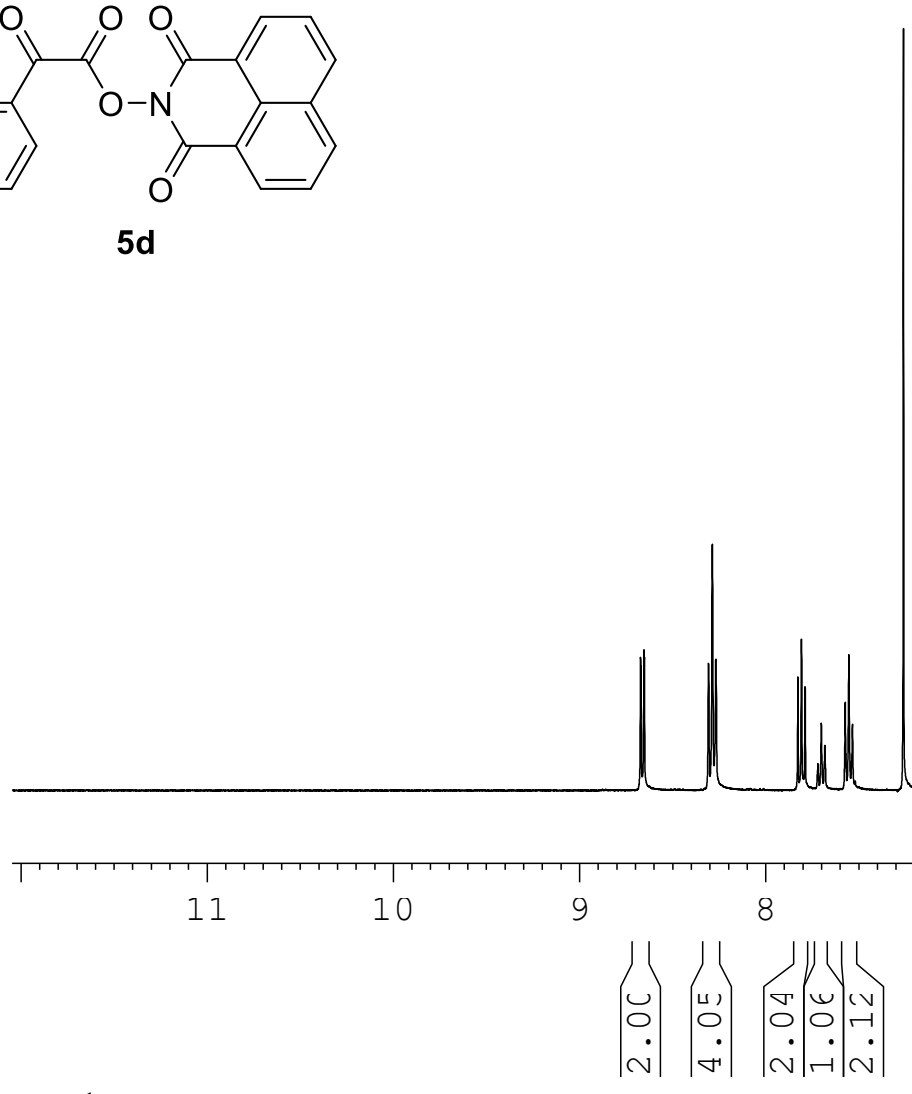

Figure S97. ${ }^{1} \mathrm{H}$ NMR spectra of $\mathbf{5 d}\left(\mathrm{CDCl}_{3}, 400 \mathrm{M}\right)$. 

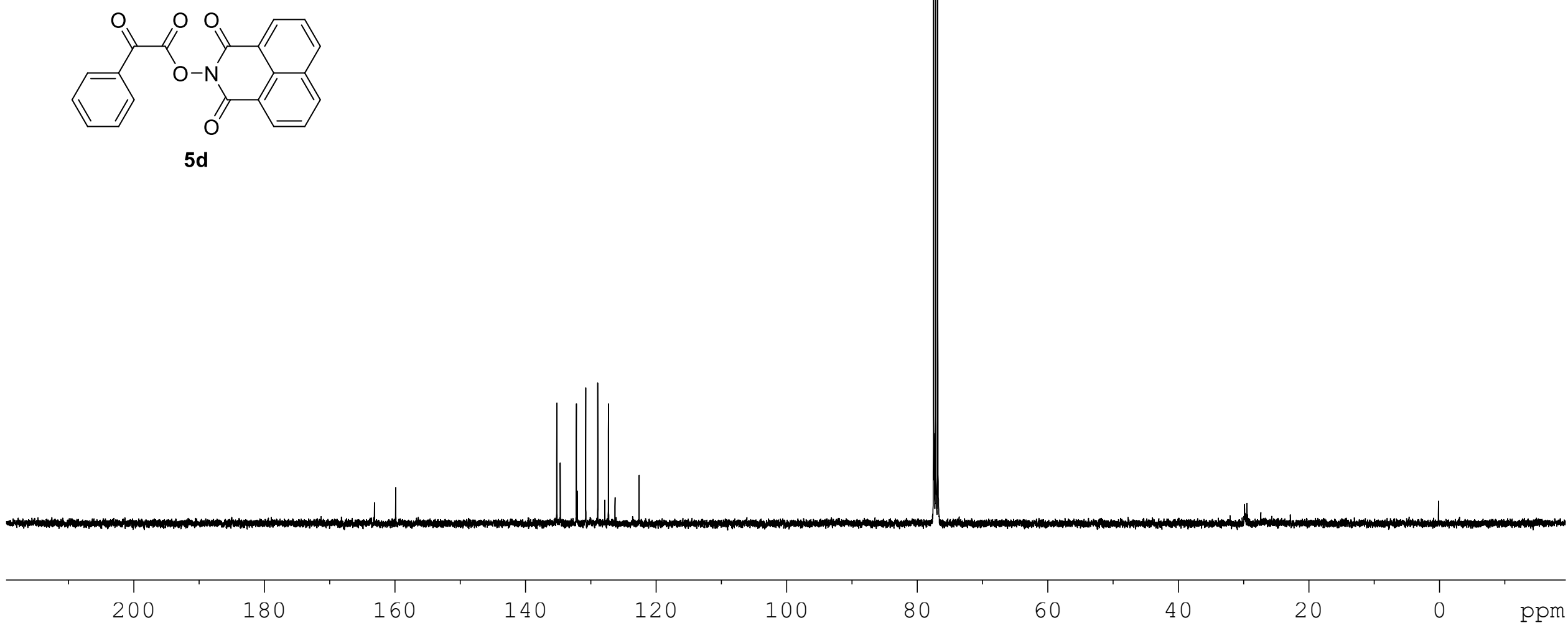

Figure $\mathrm{S} 98 .{ }^{13} \mathrm{C}$ NMR spectra of $\mathbf{5 d}\left(\mathrm{CDCl}_{3}, 100 \mathrm{M}\right)$. 